This document is downloaded from DR-NTU (https://dr.ntu.edu.sg) Nanyang Technological University, Singapore.

Distance metric learning for visual recognition

Hu, Junlin

2018

http://hdl.handle.net/10356/73159

https://doi.org/10.32657/10356/73159 


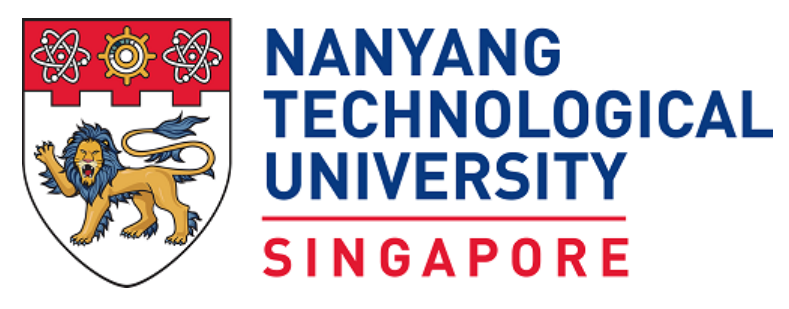

\title{
Distance Metric Learning for Visual Recognition
}

\author{
Junlin Hu
}

School of Electrical and Electronic Engineering

\begin{abstract}
A thesis submitted to the Nanyang Technological University in partial fulfillment of the requirement for the degree of

Doctor of Philosophy
\end{abstract}





\section{Acknowledgments}

I would like to thank my supervisor, Professor Yap-Peng Tan, for his precious guidance and grand support. I really appreciate his patience and efforts in helping me by providing me many insightful comments and useful ways of writing a good scientific paper and making effective attempts on academic research. I would also like to thank Dr. Jiwen Lu for his patient and generous advice, especially in discussing the details of technical problems and improving my technical writing. I want to thank Dr. Renliang Weng for his technical and personal suggestions in face recognition research. I also want to thank my friends in Nanyang Technological University, for sharing lots of what they see and hear with me in the ROSE Lab, canteens, campus bus, etc. I would like to acknowledge the School of Electrical and Electronic Engineering, and the ROSE Lab, Nanyang Technological University, for providing me the NTU Research Scholarship to complete my PhD study. Lastly, I would like to thank my family for their sincere love and support over many years. 



\section{Contents}

Acknowledgments . . . . . . . . . . . . . . . . . i i

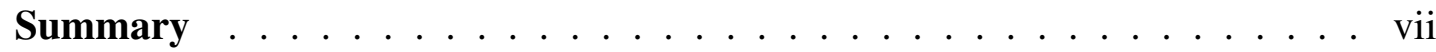

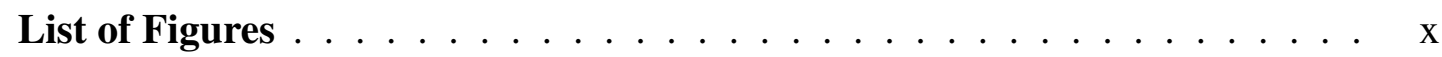

List of Tables $\ldots \ldots \ldots \ldots \ldots \ldots \ldots$. . . . . . . . . . . . . . . . . . . . .

1 Introduction 1

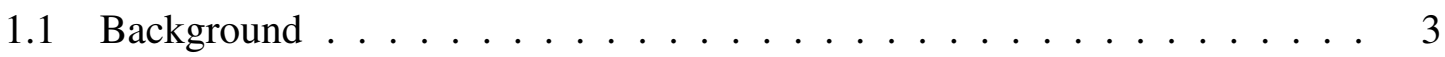

1.2 Motivation .......................... 5

1.3 Thesis Contributions . . . . . . . . . . . . . . 7

1.4 Thesis Organization . . . . . . . . . . . . . . . . . . 9

2 Literature Review $\quad 10$

2.1 Metric Learning . . . . . . . . . . . . . . . . . . . . . . . 10

2.2 Deep Metric Learning . . . . . . . . . . . . . . . . . . . . . . . 16

2.3 Deep Learning . . . . . . . . . . . . . . . . . . . . 17

2.4 Transfer Learning . . . . . . . . . . . . . . . . . . . . 17

2.5 Visual Recognition . . . . . . . . . . . . . . . . . . . . . . . 19

2.5.1 Face Verification . . . . . . . . . . . . . . . 19

2.5.2 Kinship Verification . . . . . . . . . . . . 20

2.5.3 Person Re-Identification . . . . . . . . . . . . . . 20

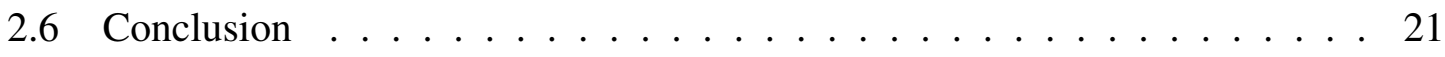


3.1 Introduction . . . . . . . . . . . . . . . . . 22

3.2 Discriminative Deep Metric Learning . . . . . . . . . . . . . . . . 23

3.2.1 Mahalanobis Distance Metric Learning . . . . . . . . . . . . . . 24

3.2.2 Discriminative Deep Metric Learning . . . . . . . . . . . . . . . 26

3.2.3 Implementation Details . . . . . . . . . . . . . . . 30

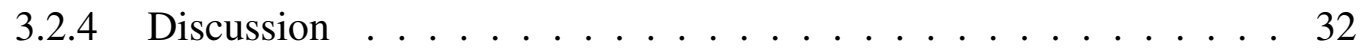

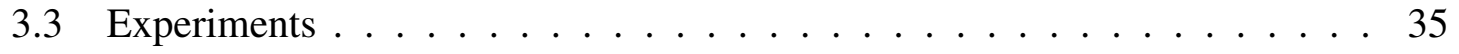

3.3 .1 Face Verification . . . . . . . . . . . 36

3.3.2 Kinship Verification . . . . . . . . . . . . . 50 50

3.3.3 Effect of the Activation Function . . . . . . . . . . . . . . 52

3.3.4 Convergence Analysis . . . . . . . . . . . . . . . . . 54

3.3.5 Computational Time . . . . . . . . . . . . . . . 55

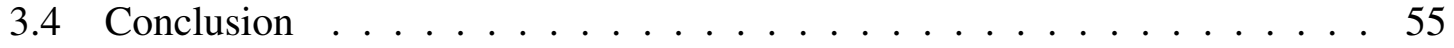

4 Deep Transfer Metric Learning $\quad 59$

4.1 Introduction . . . . . . . . . . . . . . . . . . 59

4.2 Deep Transfer Metric Learning . . . . . . . . . . . . . . . . . . . 61

4.2 .1 Notation . . . . . . . . . . . . . . . . 62

4.2 .2 Deep Metric Learning . . . . . . . . . . . . . . . . . . 62

4.2.3 Deep Transfer Metric Learning . . . . . . . . . . . . . . . . . . . 64

4.2.4 Deeply Supervised Transfer Metric Learning . . . . . . . . . . . 66

4.3 Deep Transfer Metric Learning with Autoencoder Regularization . . . . . . 69

4.3.1 DTML with Autoencoder Regularization . . . . . . . . . . . . 69

4.3.2 DSTML with Autoencoder Regularization . . . . . . . . . . . . 72 


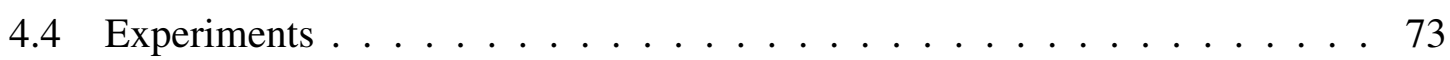

4.4 .1 Face Verification . . . . . . . . . . . . . 73

4.4.2 Person Re-Identification . . . . . . . . . . . . . . . 77

4.4.3 Handwritten Digit Recognition . . . . . . . . . . . . . . . 8 80

4.4.4 Parameter Analysis . . . . . . . . . . . . . . . . 87

4.4 .5 Discussion ........................ 90

4.5 Conclusion . . . . . . . . . . . . . . . . . 93

5 Local Large-Margin Multi-Metric Learning 95

5.1 Introduction . . . . . . . . . . . . . . . 95

5.2 Large-Margin Multi-Metric Learning . . . . . . . . . . . . . . . . 97

5.2 .1 Problem Formulation . . . . . . . . . . . . . . . . . . . 97

5.2 .2 Alternating Optimization . . . . . . . . . . . . . . 102

5.3 Local Large-Margin Multi-Metric Learning . . . . . . . . . . . . . . 104

5.3.1 Local Metric Learning . . . . . . . . . . . . . . . . . 104

5.3.2 Local Large-Margin Multi-Metric Learning . . . . . . . . . . . . 108

5.4 Experiments . . . . . . . . . . . . . . . . . . 112

5.4.1 Image-based Face Verification . . . . . . . . . . . . . . 113

5.4 .2 Video-based Face Verification . . . . . . . . . . . . . . 121

5.4 .3 Kinship Verification . . . . . . . . . . . . 126

5.4.4 Discussion and Parameter Analysis . . . . . . . . . . . 128

5.5 Conclusion . . . . . . . . . . . . . . . . . . . 134

6 Conclusion and Future Work 135

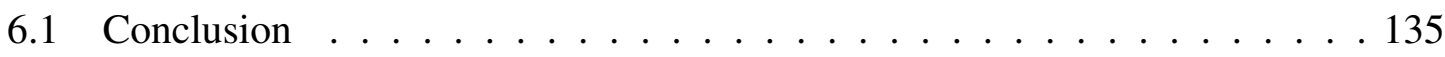

6.2 Future Work . . . . . . . . . . . . . . . . . 137 
Author's Publications

139

Bibliography

140 


\section{Summary}

How to design an effective distance function plays an important role in many computer vision and pattern recognition tasks. Over the past decade, a variety of distance metric learning algorithms have been proposed in the literature, and many of them have obtained reasonable success in various visual recognition applications, such as face recognition, image classification, person re-identification and visual search. For better efficiency, however, a number of issues remain to be addressed in distance metric learning. In this thesis, we propose several deep metric leaning methods and multi-view metric learning methods, and apply them to various visual recognition applications to demonstrate their effectiveness.

Firstly, most metric learning methods seek a single linear transformation to map data points into a new feature space; hence they are not effective enough in exploiting the nonlinear relationship of data points. Even if the kernel trick is employed to map data points into a high-dimensional feature space under which a discriminative distance metric is learned, the kernel-based metric learning methods still suffer from the scalability problem as they cannot obtain the explicit nonlinear mapping functions. To explicitly address both the nonlinearity and scalability problems, we propose a discriminative deep metric learning (DDML) method by using a deep neural network architecture. The DDML trains a neural network which learns a set of hierarchical nonlinear transformations to project data pairs into the same feature subspace, under which the distance of each positive pair is less than a small threshold and that of each negative pair is higher than a large threshold, 
respectively, so that more discriminative information can be used in the neural network. We have conducted extensive experiments on face verification and kinship verification to demonstrate the efficacy of the proposed method.

Secondly, most existing metric learning methods usually assume that the training and test samples are from the same distribution. This assumption doesn't necessarily hold in many real-world visual recognition applications. To address this problem, we propose a deep transfer metric learning (DTML) method to learn a set of hierarchical nonlinear transformations for cross-domain visual recognition by transferring discriminative knowledge from the labeled source domain to the unlabeled target domain. Specifically, the proposed DTML learns a deep metric network by maximizing the inter-class variations and minimizing the intra-class variations, and minimizing the distribution divergence between the source domain and the target domain at the top layer of the network. To better exploit the discriminative information from the source domain, we further develop a deeply supervised transfer metric learning (DSTML) method by including an additional objective on DTML, where the output of both the hidden layers and the top layer are optimized jointly. To preserve the local manifold of input data points in the metric space, we present two new methods, DTML with autoencoder regularization (DTML-AE) and DSTML with autoencoder regularization (DSTML-AE). Experimental results on face verification, person re-identification, and handwritten digit recognition validate the effectiveness of the proposed methods.

Thirdly, it is desirable to learn distance metrics from multiple feature representations so that more discriminative information can be exploited. To explore multiple feature representations in metric learning, we first propose a large-margin multi-metric learning $\left(\mathrm{LM}^{3} \mathrm{~L}\right)$ method to collaboratively learn multiple distance metrics from multiple feature representations of data, where one distance metric is learned for each feature and the correlations 
of different feature representations of each sample are maximized. Furthermore, under the learned metric spaces the distance of each positive pair is less than a small threshold and that of each negative pair is more than a large threshold, respectively. In addition, we also propose two local distance metric learning approaches, i.e., local metric learning (LML) and local large-margin multi-metric learning $\left(\mathrm{L}^{2} \mathrm{M}^{3} \mathrm{~L}\right)$, to better exploit the local manifold structures of data points. Experimental results on face verification and kinship verification tasks show the effectiveness of the proposed methods. 


\section{List of Figures}

1.1 An example on the MNIST dataset to illustrate how distance metric learning works, where the linear discriminant analysis method is employed. For ease of visualization, these samples are embedded into a two-dimensional feature spaces (a) and (b) by PCA and LDA, respectively. . . . . . . . . . . 2

3.1 The flowchart of the proposed DDML method for face verification. Given a pair of face images $\mathbf{x}_{1}$ and $\mathbf{x}_{2}$, we map them into the same feature subspace as $\mathbf{h}_{1}^{(2)}$ and $\mathbf{h}_{2}^{(2)}$ by using a set of hierarchical nonlinear transformations, where the similarity of their outputs at the most top level is computed and used to determine whether the face pair is from the same person or not. . . . 24

3.2 A toy example to illustrate how the Mahalanobis distance metric works. . . 26

3.3 Intuitive illustration of the proposed DDML method. . . . . . . . . . . 28

3.4 The network architectures used in DrLIM and DDML. (a) DrLIM uses the convolutional network, which comprises two convolutional layers $C_{1}$ and $C_{3}$, a subsampling layer $S_{2}$, and a fully connected layer $F_{3}$. (b) DDML uses the fully connected neural network . . . . . . . . . . . . . 34

3.5 Several positive face pairs from LFW, YTF, KinFaceW-I, and KinFaceWII datasets, respectively, where each column contains two images from one positive pair. . . . . . . . . . . . . . . . 35 
3.6 ROC comparison between our method and the state-of-the-art face verification methods on LFW under the (a) image restricted and (b) image unrestricted setting with label-free outside data, respectively. . . . . . . . . . 43

3.7 ROC curves of DDML, DeepFace and DeepID2 methods on LFW dataset under the unrestricted setting with labeled outside data. . . . . . . . . . . . 46

3.8 Some failure samples that were incorrectly verified by our DDML method on the LFW dataset under the image restricted setting, where $\mathbf{P}$ and $\mathbf{N}$ mean ground truth labels for positive and negative pairs, respectively. . . . . . . . 48

3.9 ROC curves comparison between our method and state-of-the-art face verification methods on YTF under image restricted setting. . . . . . . . . 50

3.10 ROC curves of our method and DeepFace method on the YTF dataset under image unrestricted setting. . . . . . . . . . . . . 5

3.11 Convergence curves of DDML on the (a) LFW and (b) YTF datasets under image restricted setting. . . . . . . . . . . . . . 56

4.1 The basic idea of the DTML method. For each sample in the training sets from the source domain and the target domain, we pass it to the developed deep neural network. We enforce two constraints on the outputs of all training samples at the top of the network: 1) the inter-class variations are maximized and the intra-class variations are minimized, and 2) the distribution divergence between the source domain and the target domain at the top layer of the network is minimized.

4.2 The network architecture used in our method. The input to the network is $\mathbf{x}$, and the output of the hidden layer and the top layer is $\mathbf{h}^{(1)}$ and $\mathbf{h}^{(2)}$, respectively. Here $\mathbf{W}^{(m)}$ and $\mathbf{b}^{(m)}$ are the parameters of the network to be learned, $1 \leq m \leq 2 \ldots \ldots \ldots \ldots \ldots \ldots \ldots$ 
4.3 The network architecture of the autoencoder used in our methods. The $\mathbf{x}$ is a data point in the input space, $\mathbf{h}^{(M)}$ is the resulting representation of the $\mathbf{x}$ in the metric space, and the $\widehat{\mathbf{x}}=\mathbf{h}^{(2 M)}$ is the reconstruction of the point $\mathbf{x}$ in the output space. . . . . . . . . . . . . . . 70

4.4 The ROC curves of several methods on LFW dataset. . . . . . . . . . . . 77

4.5 Example person images taken from the VIPeR, i-LIDS, CAVIAR and 3DPeS datasets, respectively, where each column shows two images belonging to the same person captured in two different cameras. . . . . . . . . . 78

4.6 Handwritten digit images are from MNIST and USPS datasets. . . . . . . . 85

4.7 The mean accuracy of the DTML, DSTML, DTML-AE and DSTML-AE methods with varying values of $\beta$ on the LFW dataset. . . . . . . . . . 88

4.8 The mean accuracy of the DTML-AE and DSTML-AE methods with varying values of $\theta_{t}$ and $\theta_{s}\left(\theta_{t}=\theta_{s}\right)$ on the LFW dataset. . . . . . . . . . 89

5.1 Illustration of our large-margin multi-metric learning $\left(\mathrm{LM}^{3} \mathrm{~L}\right)$ method for face verification, which jointly learns multiple distance metrics, one metric for each feature representations, and collaboratively optimizes the objective function over different features. (a) A training face image set; (b) The extracted $K$ different feature sets; (c) The distribution of these multiple feature representations in the Euclidean metric space; (d) The learning procedure of the $\mathrm{LM}^{3} \mathrm{~L}$; (e) The learned multiple distance metrics; (f) The test face image pairs; (g) The extracted multiple feature representations of the test face pairs; and (h) The resulting distance by fusing the multiple distance metrics learned by our method. . . . . . . . . . . . . . . . . 96

5.2 Some sample positive pairs from the LFW, YTF and KinFaceW-II datasets, where each column shows two face images of a positive pair. . . . . . . . 113 
5.3 ROC curves of our methods and several state-of-the-art methods on the LFW under category of image-restricted, label-free outside data. . . . . . . 116

5.4 ROC curves of our methods and several state-of-the-art methods on the YTF under the image restricted setting. . . . . . . . . . . . . . . 124

5.5 The value of the objective function of $\mathrm{LM}^{3} \mathrm{~L}$ and $\mathrm{L}^{2} \mathrm{M}^{3} \mathrm{~L}$ versus different number of iterations on the LFW dataset. . . . . . . . . . . . . . . . 129

5.6 The mean verification accuracy of $\mathrm{LM}^{3} \mathrm{~L}$ and $\mathrm{L}^{2} \mathrm{M}^{3} \mathrm{~L}$ versus different feature dimensions on the LFW dataset. . . . . . . . . . . . . . . 130

5.7 The mean verification accuracy of $\mathrm{LM}^{3} \mathrm{~L}$ and $\mathrm{L}^{2} \mathrm{M}^{3} \mathrm{~L}$ versus various $Q_{k}$ (i.e., number of local metrics) on the LFW dataset. . . . . . . . . . . 131

5.8 Distribution of cosine similarity of $\mathbf{x}_{i}^{k}$ (i.e., LBP) and $\mathbf{x}_{i}^{\ell}$ (i.e., DSIFT) before (Blue: Original space) and after (Red: Transformed space) our method on LFW dataset under category of image-restricted, label-free outside data. 133 


\section{List of Tables}

3.1 The dimension parameters of our DDML in our experiments. . . . . . . . . 39

3.2 Comparison (\%) with shallow metric learning method on LFW under the image restricted setting. . . . . . . . . . . . . . . . . 440

3.3 Comparison (\%) with shallow metric learning method on LFW under the image unrestricted setting with label-free outside data. . . . . . . . . . 40

3.4 Comparison (\%) with kernel metric learning method on LFW under the image restricted setting. . . . . . . . . . . . . . . . . . 41

3.5 Comparison (\%) with state-of-the-art metric learning methods using same feature on the LFW under the image restricted setting. . . . . . . . . . . . . 42

3.6 Comparison (\%) with state-of-the-art face verification methods on LFW under the image restricted setting, where NoD denotes the number of descriptors used. . . . . . . . . . . . . . . . . . 42

3.7 Comparison with state-of-the-art results on LFW under the image unrestricted setting with label-free outside data, where NoD denotes the number of descriptors used in that method. . . . . . . . . . . . . . . . . 44

3.8 Performance comparison (\%) with existing deep learning-based face verification methods on LFW. . . . . . . . . . . . . . . . . . . . 45

3.9 Comparison with DeepFace and DeepID2 on LFW dataset under the unrestricted setting with labeled outside data. . . . . . . . . . . . . 45 
3.10 Comparisons (\%) with LMNN method on the LFW dataset under the image unrestricted setting with labeled outside data. . . . . . . . . . . . . . 46

3.11 Comparison (\%) with DNLML-ISA method on the LFW dataset using the same setting. . . . . . . . . . . . . . . . . . 47

3.12 Comparison of the mean accuracy (\%) with the shallow metric learning methods on YTF under the image restricted setting. . . . . . . . . . . . . . 47

3.13 Comparison of the mean accuracy $(\%)$ with the state-of-the-art face verification methods on YTF under the image restricted setting.

3.14 Comparison with $\mathrm{CNN}$ based methods on the YTF dataset under image unrestricted setting. . . . . . . . . . . . . . . 50

3.15 Performance comparison (\%) with the shallow metric learning method on KinFaceW-I and KinFaceW-II datasets under image-restricted setting. . . . 53

3.16 Performance comparison (\%) with state-of-the-art methods on KinFaceW-I and KinFaceW-II datasets under image-restricted setting. . . . . . . . . . . 54

3.17 Comparison (\%) of different activation functions on LFW and YTF under the image restricted setting. . . . . . . . . . . . . . . . . . 54

4.1 Mean verification rate with standard error (\%) of different transfer metric learning methods on the LFW dataset. . . . . . . . . . . . . . . 75

4.2 Mean verification rate with standard error $(\%)$ of different metric learning methods with or without knowledge transfer on the LFW dataset. . . . . . . 76

4.3 Top $r$ ranked matching accuracy (\%) on the VIPeR dataset with \#test $=$ 316 testing persons. . . . . . . . . . . . . . . . 81

4.4 Top $r$ ranked matching accuracy (\%) on the i-LIDS dataset with \#test $=$ 60 testing persons. 
4.5 Top $r$ ranked matching accuracy $(\%)$ on the CAVIAR dataset with \#test $=$ 36 testing persons.

4.6 Top $r$ ranked matching accuracy $(\%)$ on the 3DPeS dataset with \#test $=$ 95 testing persons. . . . . . . . . . . . . . . . . . . 84

4.7 The average recognition accuracy $(\%)$ of several methods on MNIST and USPS datasets. Note that the arrow " $\rightarrow$ " denotes the direction from source domain to target domain. . . . . . . . . . . . . 86

4.8 The performance and time complexity of DTML versus different numbers of hidden layers on the LFW dataset. . . . . . . . . . . . . . . . . . 89

5.1 Comparisons of the mean verification accuracy (\%) with several baseline methods on the LFW under category of image-restricted, label-free outside data. . . . . . . . . . . . . . . . . . . . . . . . . . . . .

5.2 Comparisons of the mean verification accuracy (\%) with state-of-the-art results on the LFW under category of image-restricted, label-free outside data, where NoF denotes the number of feature used in each method. . . . . 117

5.3 The performance of $\mathrm{L}^{2} \mathrm{M}^{3} \mathrm{~L}$ on the LFW3D images under category of imagerestricted, label-free outside data. . . . . . . . . . . . . . . . . . . . . 119

5.4 Comparison of different methods using the same feature set on LFW dataset under category of image-restricted, label-free outside data. . . . . . . . . 120

5.5 Comparison of $\mathrm{LM}^{3} \mathrm{~L}$ and $\mathrm{L}^{2} \mathrm{M}^{3} \mathrm{~L}$ when $\lambda=0$ on $\mathrm{LFW}$ dataset under category of image-restricted, label-free outside data. . . . . . . . . . . . 121

5.6 Comparison of the mean verification accuracy $(\%)$ with baseline methods using different metric learning strategies on the YTF under the image restricted setting. . . . . . . . . . . . . . . . . . 122 
5.7 Comparisons of the mean verification accuracy with standard error (\%) with several state-of-the-art results on the YTF under the image restricted setting. . . . . . . . . . . . . . . . . . 123

5.8 Comparison with deep learning based methods on the YTF dataset under image unrestricted setting. . . . . . . . . . . . . . . . . 125

5.9 Comparisons of the mean verification accuracy (\%) with baseline methods using different metric learning strategies on the KinFaceW-II dataset. . . . . 127

5.10 Comparisons of the mean verification accuracy (\%) with several multiple metric learning methods and state-of-the-art methods on the KinFaceW-II dataset. . . . . . . . . . . . . . . . . . . 128 



\section{Chapter 1}

\section{Introduction}

Distance metric learning is a fundamental problem in machine learning, computer vision and pattern recognition, which aims to learn a distance function to better measure the similarity of data points. For example, in classification tasks, the $k$-nearest neighbor classifier adopts a distance metric to identify its nearest neighbors; and in clustering tasks, many clustering methods heavily depend on similarity between data points. It has been clearly shown that seeking a favorable distance metric can help improve the performance of these machine learning algorithms [1].

Generally, the optimal similarity function for a given computer vision problem is taskspecific because the underlying data distributions for different tasks are usually different. In this scenario, the hand-crafted distance metric such as Euclidean distance and cosine similarity may not work well, while metric learning can be employed to automatically learn a favorable distance metric from data points to approximate the underlying semantic metric. Recent advances on machine learning and computer vision have shown that learning a distance metric directly from a set of training examples can usually achieve better performance than the hand-crafted distance metrics [1-4].

To illustrate how distance metric learning works, we conducted an experiment on the MNIST dataset [5]. We sampled 150 samples from three classes of handwritten digits: '4', 


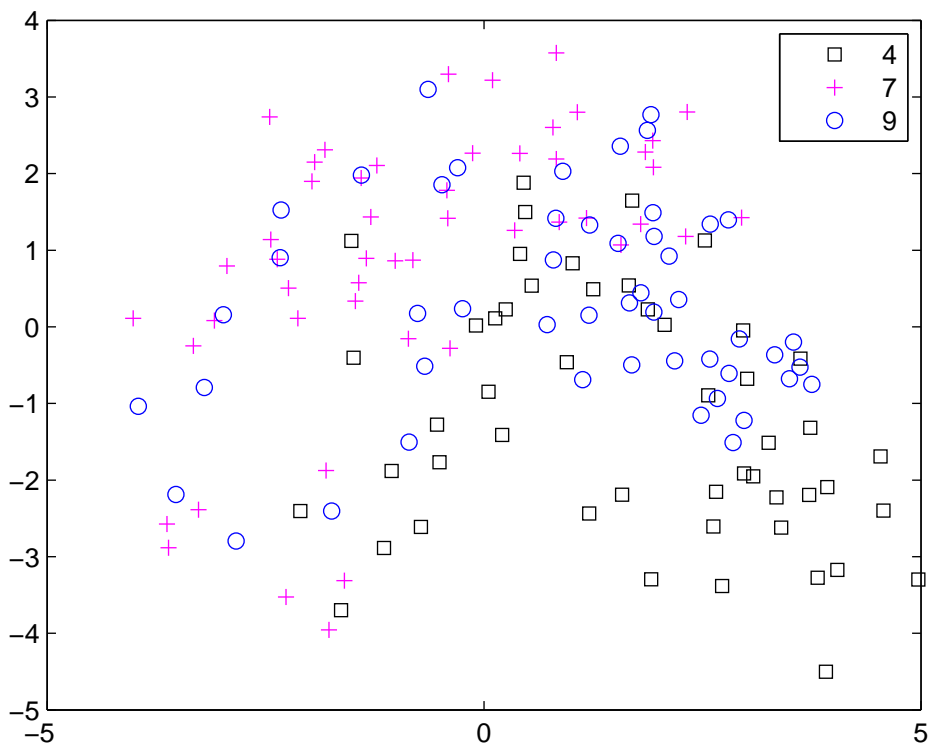

(a) The original space.

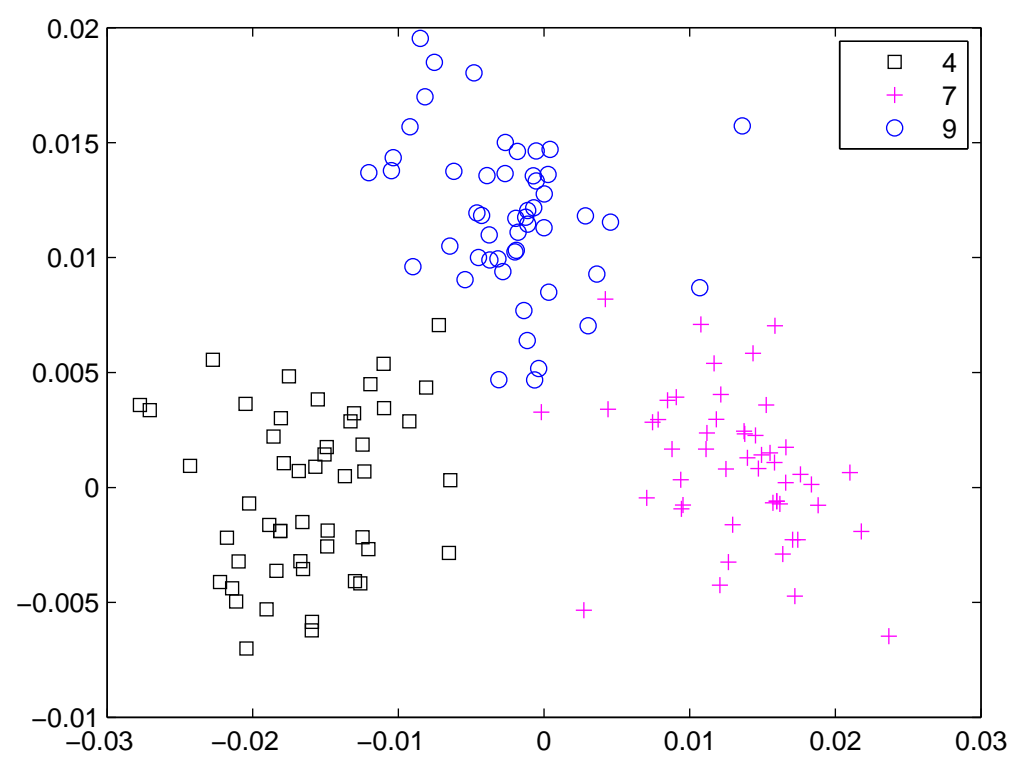

(b) The learned space.

Figure 1.1: An example on the MNIST dataset to illustrate how distance metric learning works, where the linear discriminant analysis method is employed. For ease of visualization, these samples are embedded into a two-dimensional feature spaces (a) and (b) by PCA and LDA, respectively. 
'7' and '9', where each class contains 50 samples. Each digit sample is a $28 \times 28$ gray-scale image, and we lexicographically converted it into a 784-dimensional feature vector. We employed the linear discriminant analysis (LDA) [6] as a distance metric learning method to project data points from the original space to the transformed space. Figure 1.1 shows an illustration of how distance metric learning works on this real-world dataset, where samples are respectively embedded into a 2-dimensional feature spaces (a) and (b) by principal component analysis (PCA) [6] and LDA methods. As seen, samples from different classes are mixed in the original space and they are well separated in the learned metric space.

In the following subsections, we introduce some mathematical background of distance metric learning, and present the motivation, contributions and organization of this thesis.

\subsection{Background}

To have a better understanding on distance metric learning, we briefly introduce some necessary mathematical background. This subsection simply introduces the basic definition of a metric space, and how to obtain a well-defined metric (or pseudo-metric) over the original inputs by finding a mapping into a Euclidean space.

Definition 1.1 A metric over a set $\mathcal{X}$ is a mapping $d: \mathcal{X} \times \mathcal{X} \rightarrow \mathbb{R}_{+}$and this mapping $d$ satisfies the following properties (axioms) for any $\mathbf{x}, \mathbf{y}, \mathbf{z} \in \mathcal{X}$ :

(1) $d(\mathbf{x}, \mathbf{y}) \geq 0$

(2) $d(\mathbf{x}, \mathbf{y})=d(\mathbf{y}, \mathbf{x})$,

(3) $d(\mathbf{x}, \mathbf{z}) \leq d(\mathbf{x}, \mathbf{y})+d(\mathbf{y}, \mathbf{z})$,

(4) $d(\mathbf{x}, \mathbf{x})=0$

(5) $d(\mathbf{x}, \mathbf{y})=0 \Longleftrightarrow \mathbf{x}=\mathbf{y}$. 
In Definition 1.1, the axiom (1) is called the nonnegativity axiom, the axiom (2) is known as symmetry axiom, the axiom (3) is the triangle inequality axiom, the axiom (4) is the identity axiom, and the axiom (5) is called the identity of indiscernibles axiom. A pair $(\mathcal{X}, d)$, in which $\mathcal{X}$ is a set and $d$ is a metric, is called a metric space.

Definition 1.2 A pseudo-metric over a set $\mathcal{X}$ is a mapping $d: \mathcal{X} \times \mathcal{X} \rightarrow \mathbb{R}_{+}$satisfying the following properties (axioms) for any $\mathbf{x}, \mathbf{y}, \mathbf{z} \in \mathcal{X}$ :

(1) $d(\mathbf{x}, \mathbf{y}) \geq 0$

(2) $d(\mathbf{x}, \mathbf{y})=d(\mathbf{y}, \mathbf{x})$,

(3) $d(\mathbf{x}, \mathbf{z}) \leq d(\mathbf{x}, \mathbf{y})+d(\mathbf{y}, \mathbf{z})$,

(4) $d(\mathbf{x}, \mathbf{x})=0$.

A pair $(\mathcal{X}, d)$, in which $\mathcal{X}$ is a set and $d$ is a pseudo-metric, is called a pseudo-metric space. Specifically, the pseudo-metric doesn't need to satisfy the identity of indiscernibles axiom of the metric. In distance metric learning, we may consider pseudo-metrics sometimes instead of metrics and simply refer to all of them as metrics.

The Euclidean distance metric is a widely used metric, which is usually adopted to measure the dissimilarity of data points. Given two data points $\mathrm{x}$ and $\mathbf{y}$ of $\mathcal{X}$, the Euclidean distance between $\mathbf{x}$ and $\mathbf{y}$ is defined as

$$
d(\mathbf{x}, \mathbf{y})=\|\mathbf{x}-\mathbf{y}\|_{2}=\sqrt{(\mathbf{x}-\mathbf{y})^{T}(\mathbf{x}-\mathbf{y})}
$$

in which a large distance means the dissimilarity of $\mathbf{x}$ and $\mathbf{y}$, and a small distance denotes the similarity of $\mathbf{x}$ and $\mathbf{y}$.

The main objective of distance metric learning is to learn an efficient distance metric over the input data points. One widely used method is to first map the input data points of 
the original space into a Euclidean metric space and then compute the Euclidean distance after the mapping. The following lemma depicts this method.

Lemma 1.1 Let $\mathcal{X}=\{\mathbf{x}, \mathbf{y}, \mathbf{z}, \cdots\}$ be a set, $f: \mathcal{X} \rightarrow \mathbb{R}^{n}$ be any well-defined mapping, and $d: \mathbb{R}^{n} \times \mathbb{R}^{n} \rightarrow \mathbb{R}_{+}$be the Euclidean metric over $\mathbb{R}^{n}$, then $d_{f}: \mathcal{X} \times \mathcal{X} \rightarrow \mathbb{R}_{+}$defined by $d_{f}(\mathbf{x}, \mathbf{y})=d(f(\mathbf{x}), f(\mathbf{y}))=\|f(\mathbf{x})-f(\mathbf{y})\|_{2}$ is a well-defined pseudo-metric over $\mathcal{X}$.

As Definition 1.2 (pseudo-metric) is applicable to data points $f(\mathbf{x}), f(\mathbf{y}), f(\mathbf{z})$ of $\mathbb{R}^{n}$ and it is independent of the selection of mapping $f$, Lemma 1.1 is verified.

With Lemma 1.1, distance metric learning is the procedure of learning the mapping function $f$. In addition, from the perspective of feature representation, the goal of metric learning can be interpreted as finding a new feature representation $\mathbf{h}=f(\mathbf{x})$ of the data point $\mathrm{x}$ to better suit the Euclidean space.

Thus, the objective of distance metric learning is to find optimal mapping $f$ under various loss functions and constraints, which is the principal aim of this thesis.

\subsection{Motivation}

Over the past decade, a variety of metric learning algorithms have been proposed in the literature [2-4,7-12], and some of them have been successfully applied in visual analysis applications such as face recognition [7,10,11], image classification [3,4], human activity recognition [13], person re-identification [9] and visual search [14]. These methods can be mainly classified into two categories: unsupervised and supervised. For the first category, a low-dimensional subspace or manifold is learned to preserve the geometrical information of the samples. For the second category, a discriminative distance metric is learned to maximize the separability of samples from different classes. Since the label information 
of training samples is used, supervised metric learning methods are more suitable for the visual recognition task.

While many distance metric learning algorithms have been introduced and obtained the reasonable success in many visual recognition applications, there are still several issues to be addressed for better efficiency.

- Most conventional metric learning methods usually seek a single linear transformation to map data points into a new feature space, and then compute Euclidean distance or other distance measures between data points in this new space. In this scenario, these methods may not be powerful enough to capture and exploit the nonlinear relationship of data points. To address this nonlinearity issue, the kernel trick [15] is usually adopted to map data points into a high-dimensional feature space under which a discriminative distance metric is learned by employing conventional metric learning algorithms. However, the kernel-based metric learning methods suffer from the scalability problem because they cannot obtain the explicit nonlinear mapping functions.

- Most existing metric learning methods usually assume that the training and test samples are captured under similar scenarios and that their distributions are the same. The assumptions do not necessarily hold in many real visual recognition applications, especially when samples are captured across different datasets, environments, sessions, etc. In addition, how to learn a suitable distance metric on the training datasets which consist of labeled source dataset and unlabeled target dataset and fitting the metric to the unlabeled target dataset is another challenging problem in metric learning.

- It is possible to extract multiple feature representations for each image in various visual analysis applications. It is desirable to learn distance metrics from these multiple 
feature representations such that more discriminative information can be exploited than those learned a single distance metric from a single type of features. A widely used solution is to concatenate different features together into a new feature vector and then employ existing distance metric learning algorithms on this concatenated vector directly. However, this concatenation is not physically meaningful because each feature has its own statistical characteristic, and such a simple concatenation ignores the diversity of multiple features and cannot effectively explore the complementary information among the multiple features.

\subsection{Thesis Contributions}

In this thesis, to address the aforementioned problems in distance metric learning, we propose several deep metric leaning methods and multi-view metric learning methods. The main contributions of this thesis are summarized as follows.

Firstly, to explicitly address both the nonlinearity and scalability problems, we propose a discriminative deep metric learning (DDML) method by using deep neural network architecture. The DDML trains a neural network which learns a set of hierarchical nonlinear transformations to project sample pairs into the same feature subspace, under which the distance of each positive sample pair is less than a small threshold and that of each negative pair is higher than a large threshold respectively, such that more discriminative information can be exploited in the neural network. Extensive experiments for face verification and kinship verification applications on four challenging benchmark datasets demonstrate the efficacy of the proposed method. (Chapter 3)

Secondly, to address the nonlinearity issue and the fact that the training samples and test samples are usually from different distributions in many real-world visual recognition applications, we propose a deep transfer metric learning (DTML) method to learn a set of 
hierarchical nonlinear transformations for cross-domain visual recognition by transferring discriminative knowledge from the labeled source domain to the unlabeled target domain. Specifically, the proposed DTML learns a deep metric network by maximizing the interclass variations and minimizing the intra-class variations, and minimizing the distribution divergence between the source domain and the target domain at the top layer of the neural network. To better exploit the discriminative information from the source domain, we further develop a deeply supervised transfer metric learning (DSTML) method by including an additional objective on DTML, where the output of both the hidden layers and the top layer are optimized jointly. To preserve the local manifold of input data points in the metric space, we present two new methods, DTML with autoencoder regularization (DTML-AE) and DSTML with autoencoder regularization (DSTML-AE). Experimental results on face verification, person re-identification, and handwritten digit recognition validate the effectiveness of the proposed methods. (Chapter 4)

Thirdly, to explore multiple feature representations in distance metric learning, we propose a large-margin multi-metric learning $\left(\mathrm{LM}^{3} \mathrm{~L}\right)$ method. Unlike the methods of learning a distance metric on the concatenated feature vectors, we collaboratively learn multiple distance metrics from multiple feature representations of data, where one distance metric is learned for each feature and the correlations of different feature representations of each sample are maximized, and under the learned metric spaces the distance of each positive pair is less than a small threshold and that of each negative pair is more than a large threshold, respectively. In addition, we also propose two local distance metric learning approaches, i.e., local metric learning (LML) and local large-margin multi-metric learning $\left(\mathrm{L}^{2} \mathrm{M}^{3} \mathrm{~L}\right)$, to better exploit the local manifold structures of data points. Experimental results on face verification and kinship verification tasks show that the proposed multi-view metric leaning methods can obtain competitive results compared with state-of-the-art methods. (Chapter 5) 


\subsection{Thesis Organization}

The rest of this thesis is organized as follows.

- In Chapter 2, we review the related work on metric learning, deep learning, transfer learning, and several visual recognition applications.

- In Chapter 3, we present our discriminative deep metric learning (DDML) method for face verification and kinship verification under unconstrained setting.

- Chapter 4 introduces the proposed deep transfer metric learning (DTML), deeply supervised transfer metric learning (DSTML), DTML with autoencoder regularization (DTML-AE), and DSTML with autoencoder regularization (DSTML-AE) method$\mathrm{s}$ for cross-dataset face verification, person re-identification, and handwritten digit recognition.

- In Chapter 5, we present the proposed large-margin multi-metric learning $\left(\mathrm{LM}^{3} \mathrm{~L}\right)$, local metric learning (LML), and local large-margin multi-metric learning $\left(\mathrm{L}^{2} \mathrm{M}^{3} \mathrm{~L}\right)$ methods for face verification and kinship verification.

- Finally, Chapter 6 concludes this thesis and presents several future works. 


\section{Chapter 2}

\section{Literature Review}

In this chapter, we review relevant works on metric learning, deep metric learning, deep learning, transfer learning, and a number of visual recognition applications.

\subsection{Metric Learning}

Many metric learning algorithms have been proposed over the past decade, and some of them have been successfully applied to visual analysis applications such as face recognition $[7,10,12,16]$, image classification $[3,4,17]$, person re-identification [9] and visual search [14]. Among them, most metric learning algorithms are formulated as learning a Mahalanobis metric. One of the earliest methods for Mahalanobis distance metric learning was proposed by Xing et al. [2] for clustering, named as MMC. This approach aims to minimize the sum of distances between similar data points while maximizing the sum of distances between dissimilar data points for clustering with side-information. Weinberger et al. [4] introduced a large margin nearest neighbors (LMNN) algorithm to learn a Mahanalobis distance metric for improving $k$-nearest neighbors classification, which is one of the widely-used metric learning methods. Information theoretic metric learning (ITML) [3] utilizes the relationship between the multivariate Gaussian distribution and the set of Mahalanobis distances to generalize the standard Euclidean distance. KISS metric learn- 
ing (KISSME) [9] considers learning a simple and straightforward distance metric from a statistical inference perspective, which makes the statistical decision on whether or not a pair of data points is negative by a likelihood ratio test. Unlike these Mahanalobis distance metric learning algorithms, cosine similarity metric learning (CSML) [8] aims to learn a linear transformation and calculate cosine similarity of data points in the transformed subspace. Generally, the common objective of these methods is to learn a good distance metric so that the distance between positive (similar) sample pairs is reduced and that of negative (dissimilar) sample pairs is enlarged as much as possible. More existing metric learning methods can be found in the survey [1]. We shall briefly elaborate several representative distance metric learning methods below.

ITML Given a training set $\mathbf{X}=\left[x_{1}, x_{2}, \cdots, x_{N}\right] \in \mathbb{R}^{q \times N}$ consisting of $N$ data points, the goal of conventional distance metric learning methods is to look for a positive semidefinite (PSD) matrix $\mathbf{M} \in \mathbb{R}^{q \times q}$ so that the squared Mahalanobis distance between two data points $\mathbf{x}_{i}$ and $\mathbf{x}_{j}$ is calculated as:

$$
d_{\mathbf{M}}^{2}\left(\mathbf{x}_{i}, \mathbf{x}_{j}\right)=\left(\mathbf{x}_{i}-\mathbf{x}_{j}\right)^{T} \mathbf{M}\left(\mathbf{x}_{i}-\mathbf{x}_{j}\right)
$$

where $q$ is the dimension of the input sample $\mathbf{x}_{i}$.

Information-Theoretic Metric Learning (ITML) [3] utilizes the relationship between the multivariate Gaussian distribution and the set of Mahalanobis distances to generalize the standard Euclidean distance. The idea of ITML is to seek a distance metric M to be close to a prior metric $\mathrm{M}_{0}$ by minimizing the LogDet divergence between these two matrices under the constraints that the distance $d_{\mathbf{M}}^{2}\left(\mathbf{x}_{i}, \mathbf{x}_{j}\right)$ between a positive pair is smaller than a upper bound $\tau_{p}$ while that of negative pair is larger than a lower bound $\tau_{n}$, where $0<\tau_{p}<\tau_{n}$. 
Specifically, ITML is formulated as the following LogDet optimization problem:

$$
\begin{aligned}
& \min _{\mathbf{M}} D_{l d}\left(\mathbf{M}, \mathbf{M}_{0}\right)=\operatorname{tr}\left(\mathbf{M} \mathbf{M}_{0}^{-1}\right)-\log \operatorname{det}\left(\mathbf{M M}_{0}^{-1}\right)-q \\
& \text { s.t. } d_{\mathbf{M}}^{2}\left(\mathbf{x}_{i}, \mathbf{x}_{j}\right) \leq \tau_{p} \forall \ell_{i j}=1 \\
& d_{\mathbf{M}}^{2}\left(\mathbf{x}_{i}, \mathbf{x}_{j}\right) \geq \tau_{n} \forall \ell_{i j}=-1,
\end{aligned}
$$

where the prior $\mathbf{M}_{0}$ is usually set to the identity matrix; $\operatorname{tr}(\mathbf{Z})$ is the trace of matrix $\mathbf{Z}$; $\operatorname{det}(\mathbf{Z})$ is the determinant of $\mathbf{Z}$; $\log (z)$ is the natural $\log$ arithm of $z$; and $\ell_{i j}$ denotes pairwise label between a pair of $\mathbf{x}_{i}$ and $\mathbf{x}_{j}$, which is set as $\ell_{i j}=1$ for a positive pair and $\ell_{i j}=-1$ for a negative pair. To optimize the problem (2.2), Bregman projections are iteratively computed to project the current solution onto a single constraint with the update:

$$
\mathbf{M}_{t+1}=\mathbf{M}_{t}+\beta \mathbf{M}_{t}\left(\mathbf{x}_{i}-\mathbf{x}_{j}\right)\left(\mathbf{x}_{i}-\mathbf{x}_{j}\right)^{T} \mathbf{M}_{t}
$$

where $\beta$ is the projection parameter dominated by the pairwise label and the learning rate.

KISSME KISS Metric Learning (KISSME) [9] considers learning a simple and straightforward distance metric from a statistical inference perspective, which makes the statistical decision on whether or not a sample pair $\mathbf{x}_{i}$ and $\mathbf{x}_{j}$ is negative by a likelihood ratio test. It states that the pair is negative under hypothesis $\mathcal{H}_{0}$, and that the pair is positive under hypothesis $\mathcal{H}_{1}$. The log-likelihood ratio is given as:

$$
\delta\left(\mathbf{x}_{i}, \mathbf{x}_{j}\right)=\log \left(\frac{p\left(\mathbf{x}_{i}, \mathbf{x}_{j} \mid \mathcal{H}_{0}\right)}{p\left(\mathbf{x}_{i}, \mathbf{x}_{j} \mid \mathcal{H}_{1}\right)}\right)
$$

where $p\left(\mathbf{x}_{i}, \mathbf{x}_{j} \mid \mathcal{H}_{0}\right)$ is probability distribution function of a pair under $\mathcal{H}_{0}$. Hypothesis $\mathcal{H}_{0}$ is accepted if $\delta\left(\mathbf{x}_{i}, \mathbf{x}_{j}\right)$ is larger than a threshold, otherwise hypothesis $\mathcal{H}_{1}$ is validated. By assuming a single Gaussian distribution of the pairwise difference $\mathbf{z}_{i j}=\mathbf{x}_{i}-\mathbf{x}_{j}$ and relaxing the problem $(2.4), \delta\left(\mathbf{x}_{i}, \mathbf{x}_{j}\right)$ can be simplified as:

$$
\delta\left(\mathbf{x}_{i}, \mathbf{x}_{j}\right)=\left(\mathbf{x}_{i}-\mathbf{x}_{j}\right)^{T}\left(\mathbf{C}_{p}^{-1}-\mathbf{C}_{n}^{-1}\right)\left(\mathbf{x}_{i}-\mathbf{x}_{j}\right),
$$


where covariance matrices $\mathbf{C}_{p}$ and $\mathbf{C}_{n}$ are estimated as:

$$
\begin{aligned}
\mathbf{C}_{p} & =\sum_{\ell_{i j}=1}\left(\mathbf{x}_{i}-\mathbf{x}_{j}\right)\left(\mathbf{x}_{i}-\mathbf{x}_{j}\right)^{T} \\
\mathbf{C}_{n} & =\sum_{\ell_{i j}=-1}\left(\mathbf{x}_{i}-\mathbf{x}_{j}\right)\left(\mathbf{x}_{i}-\mathbf{x}_{j}\right)^{T}
\end{aligned}
$$

To obtain the Mahalanobis distance metric $\mathbf{M}$, KISSME projects $\hat{\mathbf{M}}=\mathbf{C}_{p}^{-1}-\mathbf{C}_{n}^{-1}$ onto the cone of the positive semi-definite matrix $\mathbf{M}$ by clipping the spectrum of $\hat{M}$.

SILD Side-Information based Linear Discriminant Analysis (SILD) [18] effectively exploits the weakly-supervised side-information of sample pairs to directly calculate the within-class scatter matrix $\mathbf{C}_{p}$ in (2.6) using positive pairs and the between-class scatter matrix $\mathbf{C}_{n}$ in (2.7) utilizing negative pairs. Similar to Fisher linear discriminant analysis (FLDA) [6], SILD seeks a discriminative linear projection $\mathbf{W} \in \mathbb{R}^{q \times m}$, where $m \leq q$, by solving the optimization problem:

$$
\max _{\mathbf{W}} \frac{\operatorname{det}\left(\mathbf{W}^{T} \mathbf{C}_{n} \mathbf{W}\right)}{\operatorname{det}\left(\mathbf{W}^{T} \mathbf{C}_{p} \mathbf{W}\right)}
$$

By diagonalizing $\mathbf{C}_{p}$ and $\mathbf{C}_{n}$ as

$$
\begin{aligned}
& \mathbf{C}_{p}=\mathbf{U D}_{p} \mathbf{U}^{T}, \\
& \left(\mathbf{U D}_{p}^{-1 / 2}\right)^{T} \mathbf{C}_{p}\left(\mathbf{U D}_{p}^{-1 / 2}\right)=\mathbf{I}, \\
& \left(\mathbf{U D}_{p}^{-1 / 2}\right)^{T} \mathbf{C}_{n}\left(\mathbf{U D}_{p}^{-1 / 2}\right)=\mathbf{V D}_{n} \mathbf{V}^{T},
\end{aligned}
$$

the projection matrix $\mathbf{W}$ can be obtained as:

$$
\mathbf{W}=\mathbf{U D}_{p}^{-1 / 2} \mathbf{V}
$$

where $\mathbf{U}$ and $\mathbf{V}$ are orthogonal matrices, and $\mathbf{D}_{p}$ and $\mathbf{D}_{n}$ are two diagonal matrices. It follows that the squared Euclidean distance of $\mathbf{x}_{i}$ and $\mathbf{x}_{j}$ can be computed in the transformed 
subspace as:

$$
\begin{aligned}
d_{\mathbf{W}}^{2}\left(\mathbf{x}_{i}, \mathbf{x}_{j}\right) & =\left\|\mathbf{W}^{T} \mathbf{x}_{i}-\mathbf{W}^{T} \mathbf{x}_{j}\right\|_{2}^{2} \\
& =\left(\mathbf{x}_{i}-\mathbf{x}_{j}\right)^{T} \mathbf{W} \mathbf{W}^{T}\left(\mathbf{x}_{i}-\mathbf{x}_{j}\right) \\
& =\left(\mathbf{x}_{i}-\mathbf{x}_{j}\right)^{T} \mathbf{M}\left(\mathbf{x}_{i}-\mathbf{x}_{j}\right)
\end{aligned}
$$

which is equivalent to the Mahalanobis distance in the original space, i.e., $\mathbf{M}=\mathbf{W} \mathbf{W}^{T}$.

CSML Cosine Similarity Metric Learning (CSML) [8] aims to learn a linear transformation $\mathbf{W} \in \mathbb{R}^{q \times m}$, in which $m \leq q$, to calculate cosine similarity in this transformed subspace using:

$$
\begin{aligned}
c s_{\mathbf{W}}\left(\mathbf{x}_{i}, \mathbf{x}_{j}\right) & =\frac{\left(\mathbf{W}^{T} \mathbf{x}_{i}\right)^{T}\left(\mathbf{W}^{T} \mathbf{x}_{j}\right)}{\left\|\mathbf{W}^{T} \mathbf{x}_{i}\right\|\left\|\mathbf{W}^{T} \mathbf{x}_{j}\right\|} \\
& =\frac{\mathbf{x}_{i}^{T} \mathbf{W} \mathbf{W}^{T} \mathbf{x}_{j}}{\sqrt{\mathbf{x}_{i}^{T} \mathbf{W} \mathbf{W}^{T} \mathbf{x}_{i}} \sqrt{\mathbf{x}_{j}^{T} \mathbf{W} \mathbf{W}^{T} \mathbf{x}_{j}}} .
\end{aligned}
$$

To learn W, CSML minimizes the cross validation error by formulating the objective function as:

$$
\max _{\mathbf{W}} F(\mathbf{W})=\sum_{\ell_{i j}=1} c s_{\mathbf{W}}\left(\mathbf{x}_{i}, \mathbf{x}_{j}\right)-\alpha \sum_{\ell_{i j}=-1} c s_{\mathbf{W}}\left(\mathbf{x}_{i}, \mathbf{x}_{j}\right)-\beta\left\|\mathbf{W}-\mathbf{W}_{0}\right\|^{2}
$$

where $\mathbf{W}_{0}$ is a predefined matrix; parameter $\alpha$ balances the contributions of positive and negative pairs to the margin; and $\beta$ controls the tradeoff between the margin and the regularization term $\left\|\mathbf{W}-\mathbf{W}_{0}\right\|^{2}$. Finally, a gradient based method is used to obtain the optimal W. More details of the optimization are provided in [8].

LMNN Large Margin Nearest Neighbors (LMNN) [4] is a state-of-the-art metric learning algorithm which aims to learn a distance metric to improve the classification of the $k$ nearest neighbor (KNN) classifier. In LMNN, each data point is associated with two sets 
of different data points in the nearest neighbors: the "target" neighbors and "impostor" neighbors. There are two terms of the objective function of LMNN: one is to minimize the distances between target neighbors, and the second one is to maximize the margin between "target" and "impostor" neighbors. Based on this idea (see a schematic illustration of the basic idea behind LMNN in [4]), the objective function of LMNN is formulated as:

$$
\begin{array}{ll}
\min _{\mathbf{M}} & F(\mathbf{M})=\sum_{i j} S_{i j}\left(d_{\mathbf{M}}^{2}\left(\mathbf{x}_{i}, \mathbf{x}_{j}\right)+\gamma \sum_{k}\left(1-Y_{i k}\right) h\left(\xi_{i j k}(\mathbf{M})\right)\right) \\
\text { s.t. } & \xi_{i j k}(\mathbf{M})=1+d_{\mathbf{M}}^{2}\left(\mathbf{x}_{i}, \mathbf{x}_{j}\right)-d_{\mathbf{M}}^{2}\left(\mathbf{x}_{i}, \mathbf{x}_{k}\right) \\
& \mathbf{M} \succeq \mathbf{0},
\end{array}
$$

in which matrix $\mathbf{Y}\left(Y_{i j} \in\{0,1\}\right)$ means whether the class labels $y_{i}$ and $y_{j}$ are the same $\left(Y_{i j}=1\right)$ or different $\left(Y_{i j}=0\right)$. The matrix $\mathbf{S}$ is a binary matrix whose entry $S_{i j}$ is non-zero if instance $\mathbf{x}_{j}$ is one of the $k$ same class nearest neighbors of instance $\mathbf{x}_{i} . h(x)=\max (x, 0)$ is the standard hinge loss function. $\gamma$ is a positive free parameter, which controls the relative importance of these two competing terms, and it can be chosen via cross validation. Distance $d_{\mathbf{M}}^{2}\left(\mathbf{x}_{i}, \mathbf{x}_{j}\right)=\left(\mathbf{x}_{i}-\mathbf{x}_{j}\right)^{T} \mathbf{M}\left(\mathbf{x}_{i}-\mathbf{x}_{j}\right)$ is the squared Mahalanobis distance between data points $\mathbf{x}_{i}$ and $\mathbf{x}_{j}$.

To obtain $\mathbf{M}$, a gradient descent based method is employed on the triplets $\left(\mathbf{x}_{i}, \mathbf{x}_{j}, \mathbf{x}_{k}\right)$ and the gradient can be computed by:

$$
\frac{F(\mathbf{M})}{\partial \mathbf{M}}=\sum_{i j} S_{i j}\left(\mathbf{C}_{i j}+\gamma \sum_{k}\left(1-Y_{i k}\right)\left(\mathbf{C}_{i j}-\mathbf{C}_{i k}\right) h^{\prime}\left(\xi_{i j k}(\mathbf{M})\right)\right),
$$

where $\mathbf{C}_{i j}=\left(\mathbf{x}_{i}-\mathbf{x}_{j}\right)\left(\mathbf{x}_{i}-\mathbf{x}_{j}\right)^{T}$ denotes the outer product of pairwise differences of data points $\mathbf{x}_{i}$ and $\mathbf{x}_{j}$. For each active triplet, this formulation enhances the correlation to target neighbors while reducing the correlation to impostors. The non-differentiability of $h(x)$ at $x=0$ can be usually handled by setting $h^{\prime}(0)=0$. 
In this thesis, we contribute to metric learning for better efficiency. We propose several deep metric learning methods via deep neural network architectures to effectively exploit the nonlinear relationship of data points. We present a number of deep transfer metric learning methods by transferring discriminative knowledge from the labeled source domain to the unlabeled target domain. To make use of multiple feature representations of data in metric learning, we further introduce our multi-view metric learning methods from global and local views, respectively.

\subsection{Deep Metric Learning}

Most existing metric learning methods only learn a linear transformation to map samples into a new feature space, which may not be powerful enough to capture the nonlinear manifolds. To address this limitation, the kernel trick is usually adopted to first map samples into a high-dimensional feature space and then employ conventional metric learning methods to learn a discriminative distance metric in the high-dimensional space $[15,19]$. However, these kernel-based metric learning methods cannot explicitly obtain the nonlinear mapping functions, which usually suffer from the scalability problem. More details of metric learning via linear transformations and kernel-based metric learning can be found in the survey [1].

Motivated by the fact that deep learning is an effective solution to model the nonlinearity of samples, several deep metric learning methods [11,20-41] have been proposed to obtain explicit nonlinear mapping functions in recent years. The key idea of deep metric learning is to explicitly learn a set of hierarchical nonlinear transformations to map data points into other feature space for comparing or matching by exploiting the architecture of neural networks in deep learning, which unify feature learning and distance metric learning 
into a joint learning framework. These deep metric learning methods have shown state-ofthe-art performance for various visual understanding applications including face recognition [11, 24, 26, 30], person re-identification [25, 38], image classification [31, 32, 37], image retrieval [23, 28, 42, 43], visual tracking [35, 44], product design [34], and style matching [40], etc. In this thesis, we propose a discriminative deep metric learning methods via deep neural network architecture to effectively exploit the nonlinear relationship of data points, and also propose several deep transfer metric learning methods to transfer discriminative knowledge from the source domain to the target domain.

\subsection{Deep Learning}

In recent years, deep learning has attracted much attention in computer vision and machine learning due to its superb performance in various tasks. Generally, deep learning aims to learn hierarchical feature representations directly from raw data. It has been successfully applied to many visual tasks such as image classification [45], object detection [46], action recognition [47], and face recognition [24, 48]. Many deep learning models have been proposed in recent years, and representative methods include convolutional neural networks (CNN) [5, 45], deep stacked auto-encoder [47], deep belief networks [49], and deeplysupervised nets [50]. However, most of them aim to learn feature representations via deep model rather than similarity measure. Recently, deep learning has also been used in metric learning, and several metric learning methods have been proposed [11,22,25,31,35]. These deep metric learning methods are complementary to existing deep learning methods.

\subsection{Transfer Learning}

Transfer learning aims to address the problem when the distribution of the training data from the source domain is different from that of the target domain. Over the past decades, 
a variety of transfer learning algorithms [51-54] have been proposed and they can be mainly categorized into two classes: instance-based [55] and feature-based [56]. For the first class, different weights are learned to rank the training samples in the source domain for better learning in the target domain. For the second class, a common feature space is usually learned which can transfer the information learned from the source domain to the target domain. In recent years, several transfer learning techniques have been presented and representative methods include domain transfer support vector machine $[57,58]$, transfer dimensionality reduction [59], transfer subspace learning [60,61], and transfer metric learning [62-68]. Concerning the existing transfer metric learning methods, Zha et al. [62] proposed to learn the distance metric by minimizing the divergence between the source metrics and the target metric. Zhang and Yeung $[63,64]$ introduced a method called transfer metric learning (TML) to learn the distance metric and the task covariances between the source domain and the target domain. Luo et al. [66] proposed a decomposition-based transfer distance metric learning (DTDML) method to seek a sparse combination of the base source metrics to approximate the target metric. In [67], the high-level semantic was used in the transfer metric learning framework. While some promising results can be obtained by these transfer learning methods, most of them only consider minimizing the distribution difference between the source domain and the target domain by using linear mappings or the kernel trick, which are not effective in transferring the knowledge if the distribution difference is large and the transfer functions are usually not explicitly obtained. In this thesis, we build on the idea of deep learning and propose a deep transfer metric learning method by learning a discriminative distance network with some information transferred from the source domain. 


\subsection{Visual Recognition}

In this section, we briefly introduce several visual recognition applications including face verification, kinship verification, and person re-identification.

\subsubsection{Face Verification}

Face recognition can be mainly classified into two categories: face identification and face verification. The first aims to recognize the person from a set of gallery face samples to find the most matched one to the probe sample. The second is to determine whether a given pair of face samples is from the same person or not. A variety of unconstrained face verification methods have been proposed in recent years [7,10,48,69-77], and they can be mainly classified into two categories: appearance-based and geometry-based. Since geometric features usually cannot well make use of discriminative information of human faces, appearance-based methods are more popular in unconstrained face verification. There are two key components in appearance-based face verification: feature representation and similarity measure. For feature representation, a robust hand-crafted or learned descriptor is usually employed, so that the inter-class variations are enlarged and intra-class variations are reduced. For similarity measure, an effective distance metric is usually learned from the labeled training samples, under which the similarity of positive pairs is enlarged and that of negative pairs is reduced as much as possible. Typical feature descriptors include scale-invariant feature transform (SIFT) [78], local binary pattern (LBP) [79], fisher vector faces [73], spatial face region descriptor (SFRD) [10] and discriminant face descriptor (DFD) [80]. Representative similarity measure methods include logistic discriminant metric learning (LDML) [7], cosine similarity metric learning (CSML) [8], and pairwiseconstrained multiple metric learning (PMML) [10]. In this thesis, we contribute to the 
similarly measure (i.e., metric learning) component by presenting several new deep metric learning approaches and multi-view metric leaning methods.

\subsubsection{Kinship Verification}

Kinship verification via face images has been an emerging problem in face analysis over the past five years [81-84]. Unlike face verification which aims to verify whether a pair of face samples is from the same person or not, the objective of kinship verification is to determine whether there is a kin relation between two persons from their faces. Generally, the kin is defined as a relation between two persons who are biologically related with overlapping genes. There are four representative kin relations in kinship verification: father-son (F-S), father-daughter (F-D), mother-son (M-S) and mother-daughter (M-D). There have been a few seminal studies in kinship verification in recent years [81, 85-88], and these methods can be mainly categorized into two classes: feature-based $[81,85,86,88]$ and modelbased $[83,89]$. Generally, feature-based methods aim to extract discriminative information to preserve stable kin-related characteristics. Representative methods in this category include skin color [81], histogram of gradient [81, 85, 87], Gabor wavelet [86, 87], gradient orientation pyramid [86], salient part [90], self-similarity [91], and dynamic spatiotemporal descriptor [88]. Model-based methods apply machine learning techniques to learn an effective classifier, such as metric learning [83], multiple kernel learning [86] and graphbased fusion [89]. In this thesis, we employ distance metric learning methods for kinship verification.

\subsubsection{Person Re-Identification}

Person re-identification aims to recognize person across multiple cameras without overlapping views. This task is very challenging because images of the same subject collected in 
multiple cameras are usually different due to variations of viewpoint, illumination, pose, resolution and occlusion. Over the past decade, numerous person re-identification methods have been proposed $[38,92-95]$. The main components of person re-identification methods are two parts: extracting powerful features including handcrafted features and learned features, and finding one or more favorable distance function for matching. In this thesis, we propose several deep transfer metric learning methods for person re-identification without utilizing the label information of target camera, in which only the label information of the source camera is used for leaning a distance metric.

\subsection{Conclusion}

In this chapter, we have briefly reviewed the literature of metric learning, deep metric learning, deep learning, transfer learning, and several visual recognition applications including face verification, kinship verification, and person re-identification. While many distance metric learning algorithms have been introduced and obtained some successes in a number of visual recognition applications, several issues remain to be addressed for better efficiency.

In the following Chapters 3, 4 and 5, we present our proposed deep metric leaning methods and multi-view metric learning methods to address the limitations of existing distance metric learning, and evaluate our proposed metric learning methods on various visual recognition applications including face verification, kinship verification and person re-identification. In the next chapter, we first introduce our discriminative deep metric learning (DDML) method and then apply it to face verification and kinship verification under unconstrained conditions. 


\section{Chapter 3}

\section{Discriminative Deep Metric Learning}

In the previous chapter, we have reviewed the literature of metric learning. While metric learning has achieved reasonably good performance in various visual recognition application, most existing metric learning methods aim to learn a single Mahalanobis distance metric to maximize the inter-class variations and minimize the intra-class variations, which cannot delineate the nonlinear manifold where data points usually lie on. To address this, this chapter proposes a discriminative deep metric learning (DDML) method and applies it to face verification and kinship verification under the wild conditions. The proposed DDML method trains a deep neural network to learn a set of hierarchical nonlinear transformations to project pairs of data points into the same latent feature space, under which the distance of each positive pair is reduced and that of each negative pair is enlarged, respectively. Extensive experiments for face verification and kinship verification demonstrate the efficacy of the proposed DDML method.

\subsection{Introduction}

Most conventional metric learning methods usually seek a single linear transformation to map data points into a new feature space, and then compute Euclidean distance or other

The main part of the work presented in this chapter was published in $[11,96]$. 
distance measures between data points in this new space. In this scenario, these methods may not be powerful enough to capture and exploit the nonlinear relationship of data points. To address this nonlinearity issue, the kernel trick [15] is usually adopted to map data points into a high-dimensional feature space under which a discriminative distance metric is learned by employing conventional metric learning algorithms. However, the kernelbased metric learning methods still suffer from the scalability problem because they cannot obtain the explicit nonlinear mapping functions.

In this chapter, to explicitly address both the nonlinearity and scalability problems, we propose a discriminative deep metric learning (DDML) method by using deep neural network architecture. Figure 3.1 illustrates the basic idea of the proposed DDML approach. The proposed DDML trains a neural network which learns a set of hierarchical nonlinear transformations to project sample pairs into the same feature subspace, under which the distance of each positive sample pair is less than a smaller threshold and that of each negative pair is higher than a larger threshold respectively, such that more discriminative information can be used in the neural network. Extensive experiments for face verification and kinship verification applications on the Labeled Faces in the Wild (LFW) [69], YouTube Faces (YTF) [74], KinFaceW-I [83] and KinFaceW-II [83] datasets demonstrate the efficacy of the proposed method.

\subsection{Discriminative Deep Metric Learning}

In this section, we first briefly review the conventional Mahalanobis distance metric learning, and then present the formulation of the proposed DDML method, as well as its implementation details. 


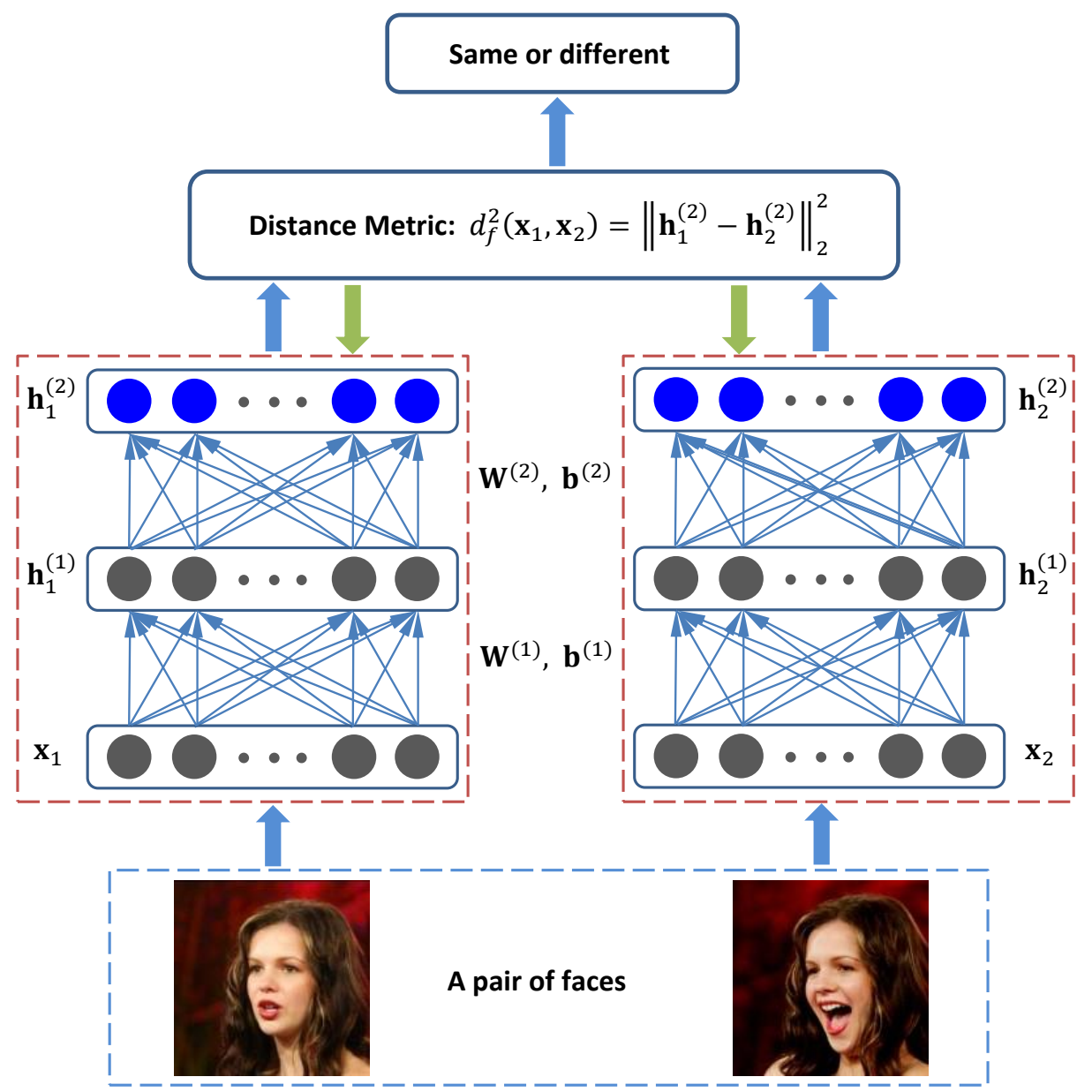

Figure 3.1: The flowchart of the proposed DDML method for face verification. Given a pair of face images $\mathbf{x}_{1}$ and $\mathbf{x}_{2}$, we map them into the same feature subspace as $\mathbf{h}_{1}^{(2)}$ and $\mathbf{h}_{2}^{(2)}$ by using a set of hierarchical nonlinear transformations, where the similarity of their outputs at the most top level is computed and used to determine whether the face pair is from the same person or not.

\subsubsection{Mahalanobis Distance Metric Learning}

Let $\mathcal{X}=\left\{\mathbf{x}_{i} \mid i=1,2, \cdots, N\right\}$ be the training set, where $\mathbf{x}_{i} \in \mathbb{R}^{d}$ is the $i$ th training sample, $N$ is the total number of training samples, and $d$ is the dimension of $\mathbf{x}_{i}$. The conventional Mahalanobis distance metric learning aims to seek a square matrix $\mathbf{M} \in \mathbb{R}^{d \times d}$ from the training set $\mathcal{X}$, under which the distance between any two samples $\mathbf{x}_{i}$ and $\mathbf{x}_{j}$ can 
be computed as:

$$
d_{\mathbf{M}}\left(\mathbf{x}_{i}, \mathbf{x}_{j}\right)=\sqrt{\left(\mathbf{x}_{i}-\mathbf{x}_{j}\right)^{T} \mathbf{M}\left(\mathbf{x}_{i}-\mathbf{x}_{j}\right)}
$$

Since $d_{\mathbf{M}}\left(\mathbf{x}_{i}, \mathbf{x}_{j}\right)$ is a distance, it should have the properties of nonnegativity, symmetry, and triangle inequality. Hence, $\mathbf{M}$ is a positive semi-definite (PSD) matrix, and it can be decomposed via eigenvalue decomposition as follows:

$$
\mathbf{M}=\mathbf{W}^{T} \mathbf{W}
$$

where $\mathbf{W} \in \mathbb{R}^{p \times d}$, and $p \leq d$.

Then, $d_{\mathbf{M}}\left(\mathbf{x}_{i}, \mathbf{x}_{j}\right)$ can be rewritten as

$$
\begin{aligned}
d_{\mathbf{M}}\left(\mathbf{x}_{i}, \mathbf{x}_{j}\right) & =\sqrt{\left(\mathbf{x}_{i}-\mathbf{x}_{j}\right)^{T} \mathbf{W}^{T} \mathbf{W}\left(\mathbf{x}_{i}-\mathbf{x}_{j}\right)} \\
& =\sqrt{\left(\mathbf{W} \mathbf{x}_{i}-\mathbf{W} \mathbf{x}_{j}\right)^{T}\left(\mathbf{W} \mathbf{x}_{i}-\mathbf{W} \mathbf{x}_{j}\right)} \\
& =\left\|\mathbf{W} \mathbf{x}_{i}-\mathbf{W} \mathbf{x}_{j}\right\|_{2} \\
& =d_{\mathbf{W}}\left(\mathbf{x}_{i}, \mathbf{x}_{j}\right) .
\end{aligned}
$$

We can see from (3.3) that learning a Mahalanobis distance metric $\mathbf{M}$ is equivalent to seeking a linear transformation $\mathbf{W}$ which projects each sample $\mathbf{x}_{i}$ into a low-dimensional subspace, under which the Euclidean distance of two samples in the transformed space is equal to the Mahalanobis distance metric in the original space.

Generally, the Mahalanobis distance metric considers the statistical regularities from training data in computing the distances of sample pairs. Geometrically, the Mahalanobis distance metric first seeks a transformation $\mathbf{W}$ to re-scale each data sample $\mathbf{x}$ as $\mathbf{z}=\mathbf{W} \mathbf{x}$ in the transformed space, under which some relevant dimensions are emphasized and some irrelevant ones are suppressed, respectively. Then, the standard Euclidean metric is applied to measure the similarity of samples in the transformed space. Figure 3.2 shows an example 


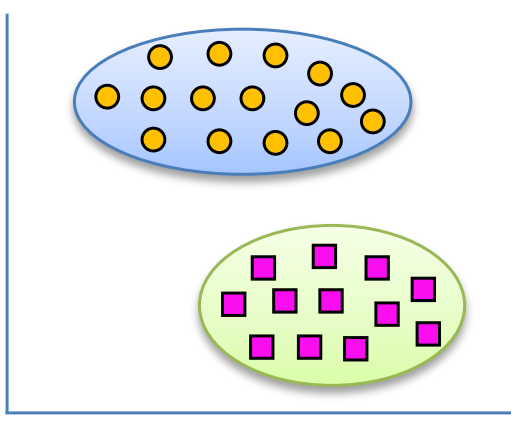

Original space

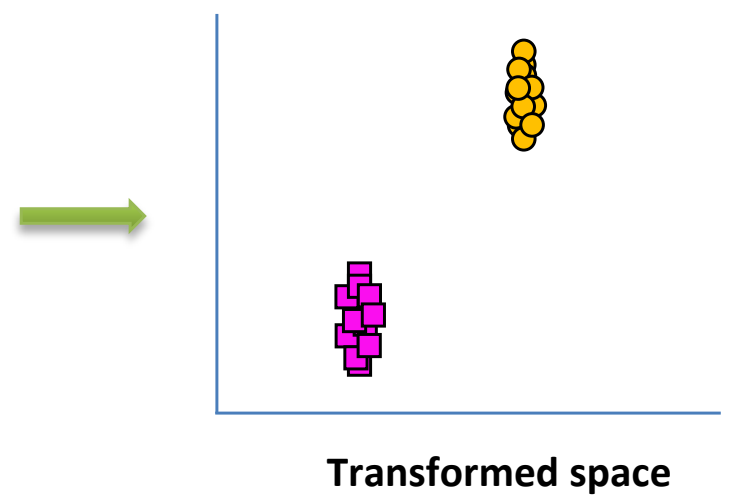

Figure 3.2: A toy example to illustrate how the Mahalanobis distance metric works.

to illustrate how the Mahalanobis distance metric works. In this figure, there are two sets of points denoted as circles and squares, and each set is located in a sphere. With the learned Mahalanobis distance metric, the feature dimension from the vertical direction is emphasized and that from the horizontal direction is compressed.

\subsubsection{Discriminative Deep Metric Learning}

The conventional Mahalanobis distance metric learning usually seeks a linear transformation, which cannot capture the nonlinear manifold of samples. To address this limitation, the kernel trick is employed to implicitly map face samples into a high-dimensional feature space and then learn a distance metric in this high-dimensional space $[15,19]$. However, these methods cannot explicitly obtain the nonlinear mapping functions, which usually suffer from the scalability problem. Different from these methods, the DDML approach is proposed to learn hierarchical nonlinear mappings to address the nonlinear and scalability problems simultaneously.

As shown in Figure 3.1, we first construct a deep neural network to compute the representations of a face image pair by passing them to multiple layers of nonlinear transformations. Assume there are $M+1$ layers in our designed network, and $p^{(m)}$ units in the $m$-th layer, where $m=1,2, \cdots, M$. For a given sample $\mathbf{x} \in \mathbb{R}^{d}$, the output of the first 
layer is $\mathbf{h}^{(1)}=\varphi\left(\mathbf{W}^{(1)} \mathbf{x}+\mathbf{b}^{(1)}\right) \in \mathbb{R}^{p^{(1)}}$, where $\mathbf{W}^{(1)} \in \mathbb{R}^{p^{(1)} \times d}$ is a projection matrix to be learned in the first layer, $\mathbf{b}^{(1)} \in \mathbb{R}^{p^{(1)}}$ is a bias vector, and $\varphi: \mathbb{R} \mapsto \mathbb{R}$ is a nonlinear activation function which operates component-wisely, e.g., the tanh or sigmoid function. Then, we use the output of the first layer $\mathbf{h}^{(1)}$ as the input of the second layer. Similarly, the output of the second layer can be computed as $\mathbf{h}^{(2)}=\varphi\left(\mathbf{W}^{(2)} \mathbf{h}^{(1)}+\mathbf{b}^{(2)}\right) \in \mathbb{R}^{p^{(2)}}$, where $\mathbf{W}^{(2)} \in \mathbb{R}^{p^{(2)} \times p^{(1)}}, \mathbf{b}^{(2)} \in \mathbb{R}^{p^{(2)}}$, and $\varphi$ are the projection matrix, bias, and nonlinear activation function of the second layer, respectively. In a similar way, the output of the $m$ th layer is $\mathbf{h}^{(m)}=\varphi\left(\mathbf{W}^{(m)} \mathbf{h}^{(m-1)}+\mathbf{b}^{(m)}\right) \in \mathbb{R}^{p^{(m)}}$, and the output of the most top level can be computed as:

$$
f(\mathbf{x})=\mathbf{h}^{(M)}=\varphi\left(\mathbf{W}^{(M)} \mathbf{h}^{(M-1)}+\mathbf{b}^{(M)}\right), \in \mathbb{R}^{p^{(M)}}
$$

where the mapping $f: \mathbb{R}^{d} \mapsto \mathbb{R}^{p^{(M)}}$ is a parametric nonlinear function which is determined by the parameters $\mathbf{W}^{(m)}$ and $\mathbf{b}^{(m)}$, where $m=1,2, \cdots, M$.

Given a pair of face samples $\mathbf{x}_{i}$ and $\mathbf{x}_{j}$, they can be finally represented as $f\left(\mathbf{x}_{i}\right)=\mathbf{h}_{i}^{(M)}$ and $f\left(\mathbf{x}_{j}\right)=\mathbf{h}_{j}^{(M)}$ at the top level when they are passed through the $M+1$-layer deep network, and their distance can be measured by computing the squared Euclidean distance between the most top level representations, which is defined as follows:

$$
d_{f}^{2}\left(\mathbf{x}_{i}, \mathbf{x}_{j}\right)=\left\|f\left(\mathbf{x}_{i}\right)-f\left(\mathbf{x}_{j}\right)\right\|_{2}^{2} .
$$

It is desirable to exploit discriminative information for feature representations of the most top level for the DDML model. To achieve this, we expect the distances between positive pairs are smaller than those between negative pairs and develop a large margin framework to formulate our method. Figure 3.3 shows the basic idea of our proposed DDML method. Specifically, the DDML aims to seek a nonlinear mapping $f$ such that the distance $d_{f}^{2}\left(\mathbf{x}_{i}, \mathbf{x}_{j}\right)$ between $\mathbf{x}_{i}$ and $\mathbf{x}_{j}$ is smaller than a pre-specified threshold $\tau_{1}$ in the 


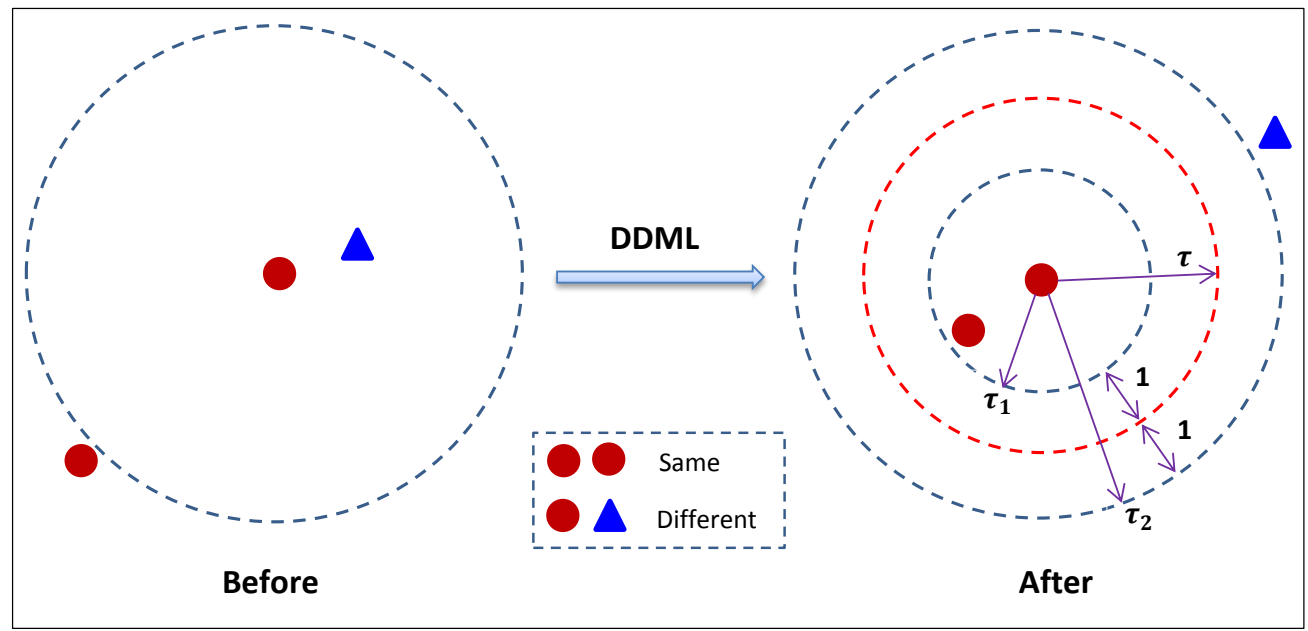

Figure 3.3: Intuitive illustration of the proposed DDML method. There are three face samples in the original feature space, which are used to generate two pairs of face images, where two of them form a positive pair (two circles) and two of them form the negative pair (one circle in the center and one triangle), respectively. In the original face feature space, the distance between the positive pair is larger than that between the negative pair which may be caused by the large intra-personal variations such as varying expressions, illuminations, and poses, especially when face images are captured in the wild. This scenario is detrimental to face verification because it causes an error. After our DDML method is applied, the distance of the positive pair is less than a smaller threshold $\tau_{1}$ and that of the negative pair is higher than a larger threshold $\tau_{2}$ of the most top level of our DDML model, respectively, so that more discriminative information can be exploited and the face pair can be easily verified.

transformed space if $\mathbf{x}_{i}$ and $\mathbf{x}_{j}$ are from the same subject $\left(\ell_{i j}=1\right)$, and larger than $\tau_{2}$ in the transformed space if samples $\mathbf{x}_{i}$ and $\mathbf{x}_{j}$ are from different subjects $\left(\ell_{i j}=-1\right)$, where the pairwise label $\ell_{i j}$ denotes the similarity or dissimilarity between a sample pair $\mathbf{x}_{i}$ and $\mathbf{x}_{j}$, and $\tau_{2}>\tau_{1}>1$.

To reduce the number of parameters in our experiments, we only employ one threshold $\tau(\tau>1)$ to connect $\tau_{1}$ and $\tau_{2}$, and enforce the margin between $d_{f}^{2}\left(\mathbf{x}_{i}, \mathbf{x}_{j}\right)$ and $\tau$ is larger than 1 by using the following constraint:

$$
\ell_{i j}\left(\tau-d_{f}^{2}\left(\mathbf{x}_{i}, \mathbf{x}_{j}\right)\right)>1
$$

where $\tau_{1}=\tau-1$ and $\tau_{2}=\tau+1$. With this constrain, there is a margin between each 
positive and negative pairs in the learned feature space, as shown in Figure 3.3.

By applying the above constrain in (3.6) to each positive and negative pair in the training set, we formulate our DDML as the following optimization problem:

$$
\arg \min _{f} J=\frac{1}{2} \sum_{i, j} g\left(1-\ell_{i j}\left(\tau-d_{f}^{2}\left(\mathbf{x}_{i}, \mathbf{x}_{j}\right)\right)\right)+\frac{\lambda}{2} \sum_{m=1}^{M}\left(\left\|\mathbf{W}^{(m)}\right\|_{F}^{2}+\left\|\mathbf{b}^{(m)}\right\|_{2}^{2}\right),
$$

where $g(z)=\frac{1}{\beta} \log (1+\exp (\beta z))$ is the generalized logistic loss function [97], which is a smoothed approximation of the hinge loss function $[z]_{+}=\max (z, 0), \beta$ is a sharpness parameter, $\|\mathbf{A}\|_{F}=\sqrt{\operatorname{tr}\left(\mathbf{A} \mathbf{A}^{T}\right)}$ represents the Frobenius norm of the matrix $\mathbf{A}$, and $\lambda$ is a positive regularization parameter. There are two terms in the objective function, the first term defines the logistic loss and the second terms represents the regularization term which is used to prevent overfitting in the model training, respectively.

To solve the optimization problem in (3.7), we use the stochastic sub-gradient descent algorithms to obtain the parameters $\left\{\mathbf{W}^{(m)}, \mathbf{b}^{(m)}\right\}$, where $m=1,2, \cdots, M$. The gradient of the objective function $J$ with respect to the parameters $\mathbf{W}^{(m)}$ and $\mathbf{b}^{(m)}$ can be calculated as follows:

$$
\begin{aligned}
\frac{\partial J}{\partial \mathbf{W}^{(m)}} & =\sum_{i, j}\left(\Delta_{i j}^{(m)} \mathbf{h}_{i}^{(m-1)^{T}}+\Delta_{j i}^{(m)} \mathbf{h}_{j}^{(m-1)^{T}}\right)+\lambda \mathbf{W}^{(m)}, \\
\frac{\partial J}{\partial \mathbf{b}^{(m)}} & =\sum_{i, j}\left(\Delta_{i j}^{(m)}+\Delta_{j i}^{(m)}\right)+\lambda \mathbf{b}^{(m)},
\end{aligned}
$$

where $\mathbf{h}_{i}^{(0)}=\mathbf{x}_{i}$ and $\mathbf{h}_{j}^{(0)}=\mathbf{x}_{j}$, which are from the original inputs of our network. For all other layers $m=1,2, \cdots, M-1$, we have the following updating equations:

$$
\begin{aligned}
& \Delta_{i j}^{(M)}=g^{\prime}(c) \ell_{i j}\left(\mathbf{h}_{i}^{(M)}-\mathbf{h}_{j}^{(M)}\right) \odot \varphi^{\prime}\left(\mathbf{z}_{i}^{(M)}\right), \\
& \Delta_{j i}^{(M)}=g^{\prime}(c) \ell_{i j}\left(\mathbf{h}_{j}^{(M)}-\mathbf{h}_{i}^{(M)}\right) \odot \varphi^{\prime}\left(\mathbf{z}_{j}^{(M)}\right), \\
& \Delta_{i j}^{(m)}=\left(\mathbf{W}^{(m+1)^{T}} \Delta_{i j}^{(m+1)}\right) \odot \varphi^{\prime}\left(\mathbf{z}_{i}^{(m)}\right), \\
& \Delta_{j i}^{(m)}=\left(\mathbf{W}^{(m+1)^{T}} \Delta_{j i}^{(m+1)}\right) \odot \varphi^{\prime}\left(\mathbf{z}_{j}^{(m)}\right),
\end{aligned}
$$


where the operator $\odot$ denotes the element-wise multiplication, and $c$ and $\mathbf{z}_{i}^{(m)}$ are defined as follows:

$$
\begin{gathered}
c \triangleq 1-\ell_{i j}\left(\tau-d_{f}^{2}\left(\mathbf{x}_{i}, \mathbf{x}_{j}\right)\right), \\
\mathbf{z}_{i}^{(m)} \triangleq \mathbf{W}^{(m)} \mathbf{h}_{i}^{(m-1)}+\mathbf{b}^{(m)} .
\end{gathered}
$$

Then, $\mathbf{W}^{(m)}$ and $\mathbf{b}^{(m)}$ can be updated by using the following gradient descent algorithm until a certain convergence condition is reached:

$$
\begin{aligned}
\mathbf{W}^{(m)} & =\mathbf{W}^{(m)}-\mu \frac{\partial J}{\partial \mathbf{W}^{(m)}}, \\
\mathbf{b}^{(m)} & =\mathbf{b}^{(m)}-\mu \frac{\partial J}{\partial \mathbf{b}^{(m)}},
\end{aligned}
$$

where $\mu$ is the learning rate.

Algorithm 1 summarizes the detailed procedure of the proposed DDML method.

\subsubsection{Implementation Details}

In this subsection, we discuss the nonlinear activation functions and the initializations of $\mathbf{W}^{(m)}$ and $\mathbf{b}^{(m)}, 1 \leq m \leq M$ in our proposed DDML method.

\subsubsection{Activation Function}

There are many nonlinearity activation functions which could be used to determine the output of the nodes in our deep metric learning model. In our experiments, we use the tanh function as the activation function because we found it achieving better performance than others in View 1 of Labeled Faces in the Wild (LFW) dataset [69]. There are two views in LFW dataset [69], View 1 and View 2. View 1 is used for model selection and algorithm development and View 2 is used for performance reporting. In View 1 of LFW, the training set contains 1100 positive pairs and 1100 negative pairs, and the test set contains 500 positive pairs and 500 negative pairs. We evaluated three nonlinear activation functions 


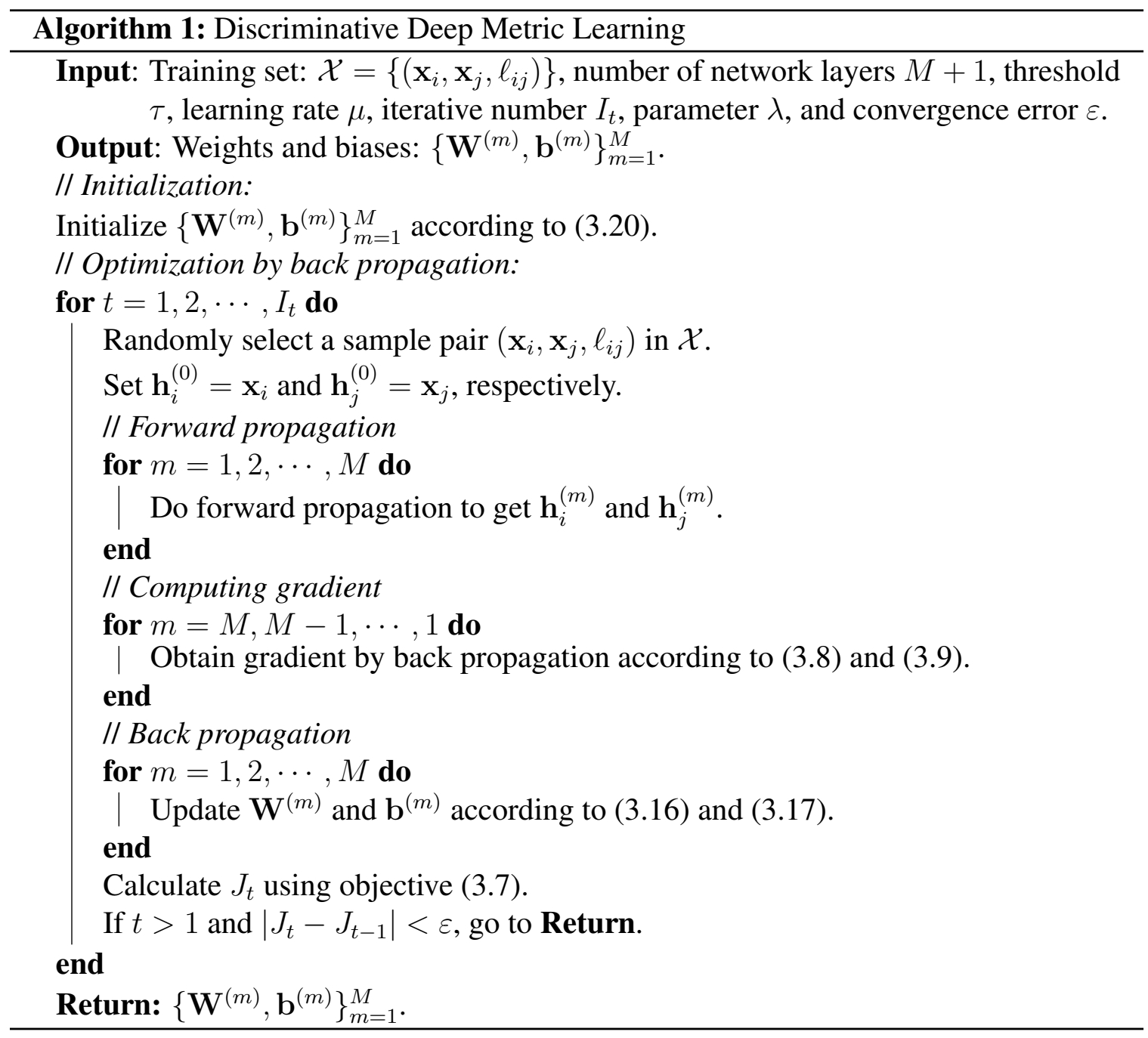

(i.e., sigmoid, non-saturating sigmoid (ns-sigmoid) ${ }^{1}$, and tanh) in View 1 of LFW. We find the tanh function reports the best accuracy in View 1 of LFW, therefore we employ the tanh function as nonlinear activation function for all datasets. The tanh function and its derivative can be computed as follows:

$$
\begin{aligned}
\varphi(z) & =\tanh (z)=\frac{e^{z}-e^{-z}}{e^{z}+e^{-z}} \\
\varphi^{\prime}(z) & =\tanh ^{\prime}(z)=1-\tanh ^{2}(z) .
\end{aligned}
$$

\footnotetext{
${ }^{1}$ The sigmoid function is defined as $s(z)=1 /\left(1+e^{-z}\right)$, and the ns-sigmoid function is defined as $z=s^{3}(z) / 3+s(z)$.
} 


\subsubsection{Initialization}

The initializations of $\mathbf{W}^{(m)}$ and $\mathbf{b}^{(m)}(1 \leq m \leq M)$ are important to the gradient descent based method in our deep neural networks. Random initialization and denoising autoencoder (DAE) [98] are two popular initialization methods in deep learning. In our experiments, we utilize a simple normalized random initialization method in [99], where the bias $\mathbf{b}^{(m)}$ is initialized as $\mathbf{0}$, and the weight of each layer is initialized as the following uniform distribution:

$$
\mathbf{W}^{(m)} \sim U\left[-\frac{\sqrt{6}}{\sqrt{p^{(m)}+p^{(m-1)}}}, \frac{\sqrt{6}}{\sqrt{p^{(m)}+p^{(m-1)}}}\right],
$$

where $p^{(0)}$ is the dimension of input layer, $1 \leq m \leq M$.

\subsubsection{Discussion}

In this subsection, we highlight the difference between our deep metric learning model and two existing deep metric learning methods, Deep Nonlinear Metric Learning via ISA Network (DNLML-ISA) [22], and Dimensionality Reduction by Learning an Invariant Mapping (DrLIM) [20,21].

DNLML-ISA [22] While both DNLML-ISA and our methods can learn nonlinear similarity measure, DNLML-ISA is a stacked ISA network based deep learning approach and ours is a fully connected nonlinear feed-forward network. Hence, there is no backpropagation in the DNLML-ISA method. However, the back-propagation strategy is used in our methods because the large margin objective is explicitly enforced in the top layer of the deep modal and the parameters of other layers are automatically learned. Therefore, our methods are more flexible for practical applications. Most previous deep learning methods have shown that the back-propagation deep model can usually yield better performance than the stacked auto-encoder based approach. Therefore, our methods could 
achieve better performance than the DNLML-ISA method. Empirical results were presented in Section 3.3.1.2 to illustrate this point.

DrLIM [20,21] While there are some similar points between our proposed DDML and the existing DrLIM method [20,21] because both of them learn nonlinear mappings to map samples from the original space into the feature space to exploit discriminative information to improve the verification performance, there are several key differences between DDML and DrLIM, which are highlighted as follows:

- DrLIM jointly learns feature representation and similarity measure in the designed network while DDML only learns the similarity measure, as shown in Figure 3.4. Hence, the input to DrLIM is original raw face images and that to DDML is handcrafted features. While convolutional neural networks $(\mathrm{CNN})$ can learn robust feature representations, these methods require a large number of labeled data for training because there are extensive number of parameters to estimate. For some practical applications such as kinship verification, it is very hard to collect such large number of labeled data for feature learning. In contrast, the number of parameters in our DDML is quite small so that it can also work well for discriminative metric learning with small size of training samples.

- The network architectures of DrLIM and DDML are different. As shown in Figure 3.4, there are several convolutional and sub-sampling (pooling) layers in DrLIM, and these layers are not used in DDML. Specifically, the network of DrLIM can be divined into two parts: feature learning (convolutional and sub-sampling layers) and similarity metric learning (fully connection layers). Hence, our DDML model is simpler than DrLIM because the complexity of the network is largely reduced. 


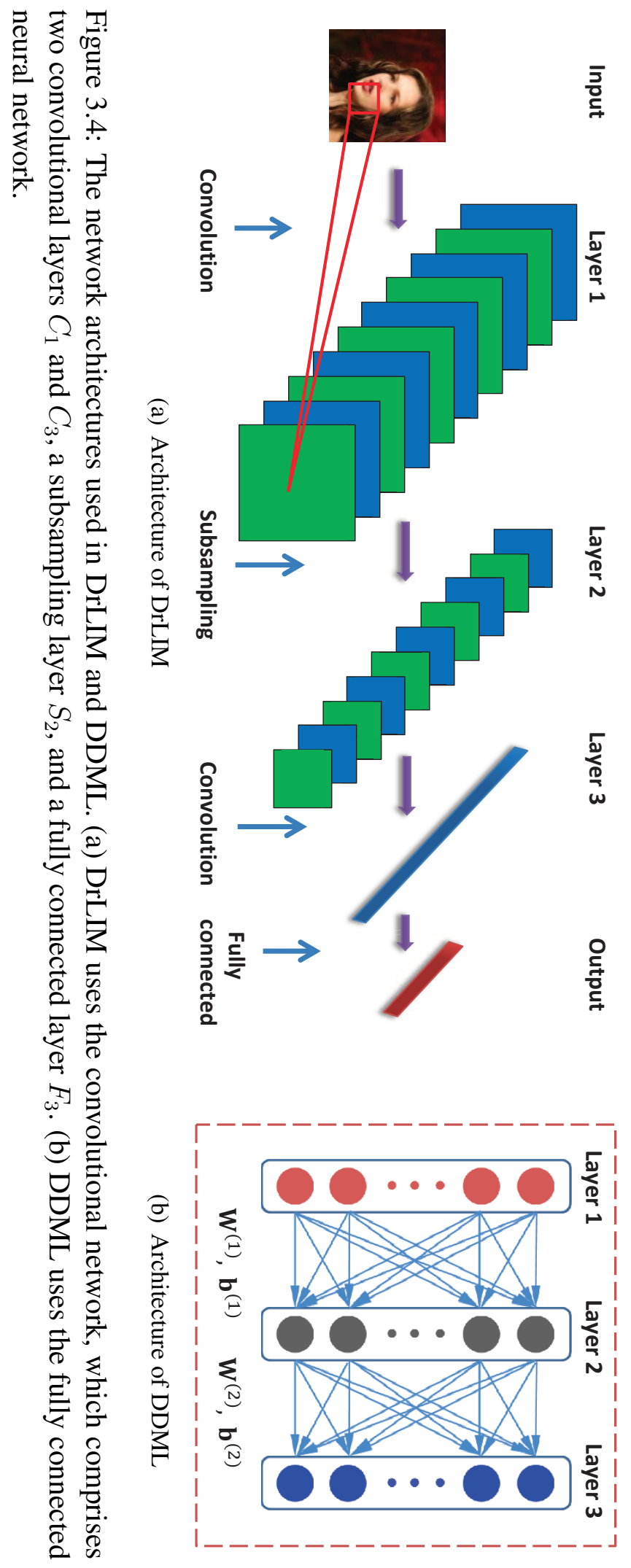




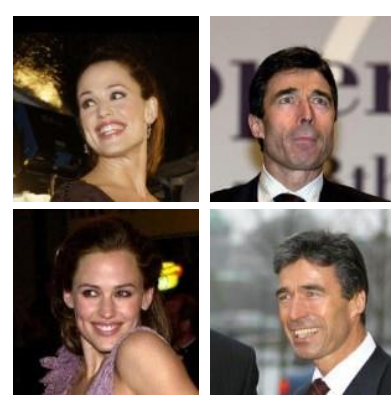

LFW
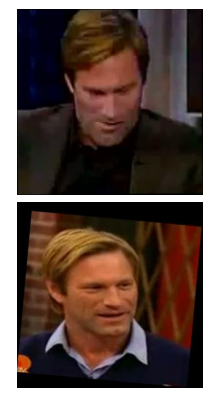

YTF

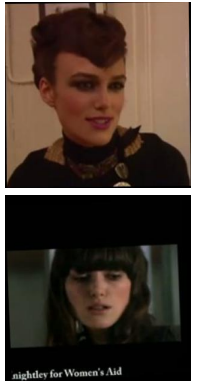

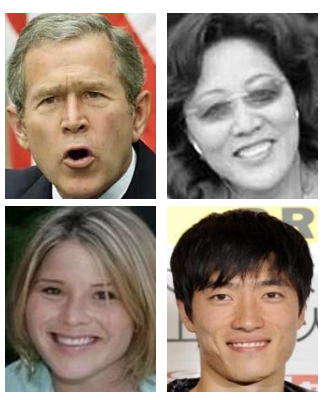

KinFaceW-I

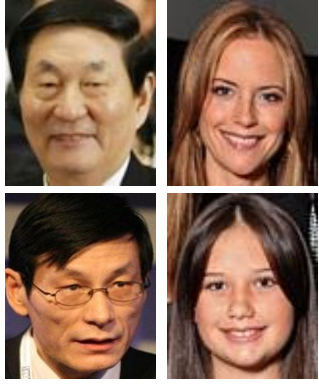

KinFaceW-II

Figure 3.5: Several positive face pairs from LFW, YTF, KinFaceW-I, and KinFaceW-II datasets, respectively, where each column contains two images from one positive pair.

Moreover, it is arguable whether deep feature learning is a must for face verification.

Since there is usually a pre-processing step for face detection and alignment in a face recognition system, face images can be roughly aligned before feature extraction. With these aligned face images, some hand-crafted features such as LBP and HOG can also well describe face images in the feature space. With the simplified network, deep model can be much easier to be analyzed. We also achieve very competitive performance on the benchmark datasets, which further demonstrates the effectiveness of the proposed approach.

- The objective functions at the top layer of DrLIM and DDML are different. DrLIM directly employs the squared hinge loss to define the objective function and DDML uses a generalized logistic loss function to approximate the hinge loss function.

\subsection{Experiments}

To evaluate the effectiveness of the proposed DDML method, we perform unconstrained face verification and kinship verification experiments on the challenging LFW [69], YouTube Face (YTF) [74], KinFaceW-I [83], and KinFaceW-II [83] datasets. Figure 3.5 shows some sample face images from these datasets. 


\subsubsection{Face Verification}

Over the past two decades, a large number of face recognition methods have been proposed in the literature, and most of them have achieved satisfying recognition performance under controlled conditions. However, their performance drops heavily when face images are captured in the wild because large intra-class variations usually occur in this scenario. Face recognition can be mainly classified into two tasks: face identification and face verification. The former aims to recognize the person from a set of gallery face images or videos and find the most similar one to the probe sample. The latter is to determine whether a given pair of face images or videos is from the same person or not. In this subsection, we consider the second one where face images contain significant variations caused by varying lighting, expression, pose, resolution, and background.

\subsubsection{Datasets and Experimental Settings}

LFW The LFW dataset [69] contains more than 13000 face images of 5749 subjects captured from the web with variations in expression, pose, age, illumination, resolution, background, and so on. There are two training paradigms for supervised learning on this dataset: 1) image restricted and 2) image unrestricted. In our experiments, we evaluate our proposed methods on both of these two settings. We follow the standard evaluation protocol on the "View 2" dataset [69] which includes 3000 matched pairs and 3000 mismatched pairs. The dataset is divided into 10 folds, and each fold consists of 300 matched (positive) pairs and 300 mismatched (negative) pairs. We use three types of the LFW dataset for feature extraction in our evaluation: the original LFW, LFW-a ${ }^{2}$, and "funneled" LFW". For the original LFW dataset, we employ the over-completed high-dimensional LBP (HDLBP)

\footnotetext{
${ }^{2}$ Available: http://www.openu.ac.il/home/hassner/data/lfwa/.

${ }^{3}$ Available: http://vis-www.cs.umass.edu/lfw/.
} 
provided by [100] as the feature representation for each image. Specifically, it densely samples multi-scale LBP features at each landmarks and then these features are concatenated into a high-dimensional feature vector (more than 120000 dimension). For the LFW-a dataset, we crop each image into $80 \times 150$ from center of this image to remove the background information and extract the histogram of oriented gradients (HOG) [101] from two different scales for each image. Specifically, we first divide each image into $16 \times 30$ nonoverlapping blocks, where the size of each block is $5 \times 5$. Then, we divide each image into $8 \times 15$ non-overlapping blocks, where the size of each block is $10 \times 10$. Subsequently, we extract a 9-dimensional HOG feature for each block and concatenate them to form a 5400-dimensional feature vector. For the "funneled" LFW dataset, we use the Sparse SIFT (SSIFT) descriptor provided by [7]. Specifically, these SSIFT descriptors are computed at the nine fixed landmarks with three different scales, and are concatenated into a 3456dimensional feature vector. As suggested in $[8,71,102]$, we also use the square root of each feature. For each feature descriptor, we apply whitened PCA (WPCA) to reduce it into an appropriate dimension to further remove the redundancy. Specifically, the WPCA projection was computed on the training data of each cross-validation split.

YTF The YTF dataset [74] contains 3425 videos of 1595 different persons collected from the YouTube website. There are large variations in pose, illumination, and expression in each video, and the average length of each video clip is 181.3 frames. In our experiments, we follow the standard evaluation protocol [74] and test our method for unconstrained face verification with 5000 video pairs. These pairs are equally divided into 10 folds, and each fold has 250 intra-personal pairs and 250 inter-personal pairs. We adopt the image restricted protocol to evaluate our method. For this dataset, we directly use the provided three feature descriptors [74] including LBP, Center-Symmetric LBP (CSLBP) [74] and 
Four-Patch LBP (FPLBP) [70]. Since all face images have been aligned by the detected facial landmarks, we average all the feature vectors within one video clip to form a mean feature vector in our experiments. Lastly, we use WPCA to project each mean vector into a vector with appropriate dimension. For both LFW and YTF datasets, we follow the standard protocol in $[69,74]$ and use two measures including the mean verification accuracy (\%) with standard error and the receiving operating characteristic (ROC) curve from the ten-fold cross validation to validate our method.

Parameter Settings For our DDML, we train a deep network with three layers $(M=2)$, and the threshold $\tau$, learning rate $\mu$ and regularization parameter $\lambda$ are empirically set as $3,10^{-3}, 10^{-2}$ for all experiments, respectively. The parameters $\left\{\mathbf{W}^{(m)}, \mathbf{b}^{(m)}\right\}_{m=1}^{M}$ of DDML were set as shown in Table 3.1. Following the standard protocol in $[69,74]$, we use two measures including the mean classification accuracy with standard error and the receiving operating characteristic (ROC) curve from ten-fold cross-validation to validate the proposed DDML method.

\subsubsection{Experimental Comparison on LFW}

Deep vs. Shallow Metric Learning We first compare our method with shallow metric learning methods. A shallow metric learning method called discriminative shallow metric learning (DSML) is constructed, where only one layer $(M=1)$ is learned in our DDML method and the activation function is $\varphi(z)=z$. DSML is the linear case of the proposed DDML, i.e., the linear activation function $\varphi(z)=z$ and no hidden layer $(M=1)$ are adopted in the DDML. Tables 3.2 and 3.3 record the verification rates with standard error of these methods when different feature descriptors are used. We see that our DDML consistently outperform DSML in terms of the mean verification rate. The reason is that DDML learn- 


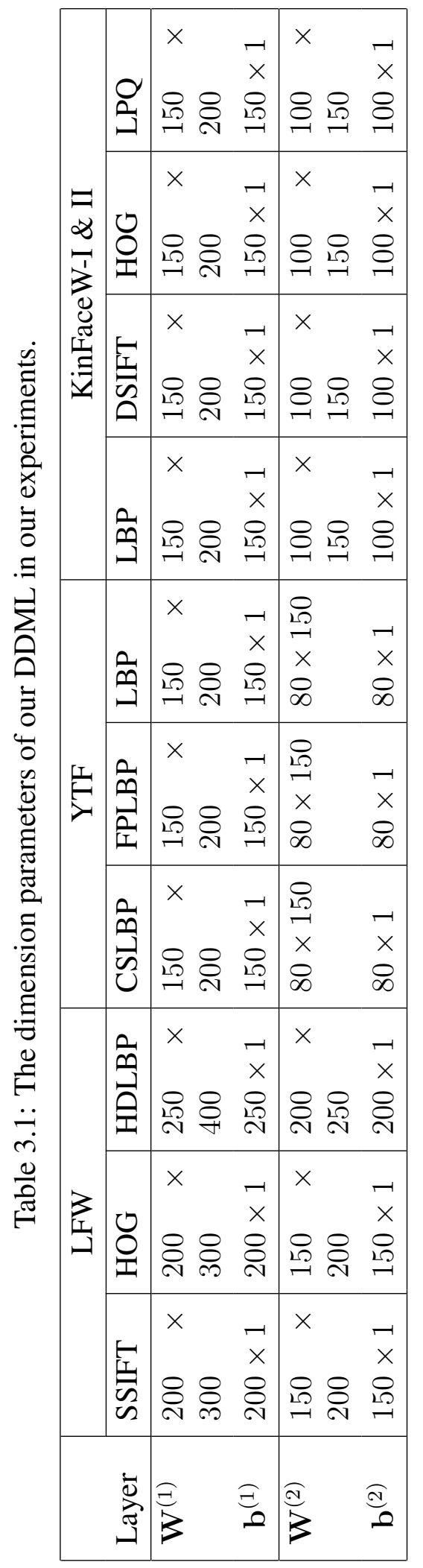


Table 3.2: Comparison (\%) with shallow metric learning method on LFW under the image restricted setting.

\begin{tabular}{|l|c|c|}
\hline Feature & DDML & DSML \\
\hline \hline SSIFT & $86.98 \pm 0.43$ & $83.67 \pm 0.35$ \\
SSIFT (sqrt) & $87.83 \pm 0.29$ & $84.55 \pm 0.29$ \\
\hline HOG & $87.35 \pm 0.58$ & $85.22 \pm 0.62$ \\
HOG (sqrt) & $88.38 \pm 0.77$ & $86.40 \pm 0.55$ \\
\hline HDLBP & $91.37 \pm 0.39$ & $88.75 \pm 0.37$ \\
HDLBP (sqrt) & $\mathbf{9 2 . 5 5} \pm \mathbf{0 . 3 6}$ & $90.47 \pm 0.28$ \\
\hline
\end{tabular}

Table 3.3: Comparison (\%) with shallow metric learning method on LFW under the image unrestricted setting with label-free outside data.

\begin{tabular}{|l|c|c|}
\hline Feature & DDML & DSML \\
\hline \hline SSIFT & $88.42 \pm 0.52$ & $85.35 \pm 0.43$ \\
SSIFT (sqrt) & $88.92 \pm 0.45$ & $85.63 \pm 0.42$ \\
\hline HOG & $88.08 \pm 0.62$ & $86.25 \pm 0.54$ \\
HOG (sqrt) & $89.18 \pm 0.69$ & $87.60 \pm 0.61$ \\
\hline HDLBP & $92.62 \pm 0.35$ & $90.05 \pm 0.35$ \\
HDLBP (sqrt) & $\mathbf{9 3 . 7 0} \pm \mathbf{0 . 3 7}$ & $91.57 \pm 0.33$ \\
\hline
\end{tabular}

s hierarchical nonlinear transformations while DSML only learns a linear transformation, such that DDML can better exploit the nonlinear relationship of face samples.

Deep vs. Kernel-based Metric Learning We employ the kernel trick on the DSML method and develop another baseline method (kernel DSML: KDSML) to map the data vectors into a high dimensional space without having to explicitly compute the mapping. More details of the KDSML method are presented in Appendix of this chapter. In our experiments, we use the RBF kernel $\kappa\left(\mathbf{x}_{i}, \mathbf{x}_{j}\right)=\exp \left(-\gamma\left\|\mathbf{x}_{i}-\mathbf{x}_{j}\right\|_{2}^{2}\right)$. Table 3.4 shows performance comparisons with KDSML method on the LFW under the image restricted setting. We see that our deep metric learning method consistently outperform the corresponding kernel metric learning method over all different feature representations. 
Table 3.4: Comparison (\%) with kernel metric learning method on LFW under the image restricted setting.

\begin{tabular}{|l|c|c|}
\hline Feature & DDML & KDSML \\
\hline \hline SSIFT & $86.98 \pm 0.43$ & $84.65 \pm 0.48$ \\
SSIFT (sqrt) & $87.83 \pm 0.29$ & $85.68 \pm 0.38$ \\
\hline HOG & $87.35 \pm 0.58$ & $85.83 \pm 0.53$ \\
HOG (sqrt) & $88.38 \pm 0.77$ & $87.02 \pm 0.43$ \\
\hline HDLBP & $91.37 \pm 0.39$ & $89.36 \pm 0.40$ \\
HDLBP (sqrt) & $\mathbf{9 2 . 5 5} \pm \mathbf{0 . 3 6}$ & $90.50 \pm 0.35$ \\
\hline
\end{tabular}

Comparison with the State-of-the-Art Metric Learning Methods We compare our proposed deep metric learning method with state-of-the-art metric learning methods on the LFW dataset under the image restricted setting. For a fair comparison, we used the same SSIFT feature so that the same setting is set for different metric learning methods. Table 3.5 shows the verification performance of different metric learning methods on the LFW dataset. We see that our proposed DDML significantly improves the previous metric learning methods. There are two reasons: 1) our method is a nonlinear metric learning approach while most previous ones are linear; and 2) our method is a deep learning approach while most previous ones are shallow models.

Comparison with the State-of-the-Art Face Verification Methods We compare our method with the state-of-the-art face verification methods on the LFW dataset under the image restricted and unrestricted settings with label-free outside data, respectively ${ }^{4}$. Tables 3.6 and 3.7 show the mean verification rate with standard error and Figure 3.6 shows the ROC curves of different methods, respectively. We see that our DDML improves these

\footnotetext{
${ }^{4}$ Available: http://vis-www.cs.umass.edu/lfw/results.html.
} 
Table 3.5: Comparison (\%) with state-of-the-art metric learning methods using same feature on the LFW under the image restricted setting.

\begin{tabular}{|l|c|}
\hline Method & Accuracy (\%) \\
\hline \hline ITML (SSIFT: sqrt) [3] & $76.20 \pm 0.50$ \\
LDML (SSIFT: sqrt) [7] & $77.50 \pm 0.50$ \\
PCCA (SSIFT: sqrt) [97] & $82.20 \pm 0.40$ \\
Kernel PCCA (SSIFT: sqrt) [97] & $83.80 \pm 0.40$ \\
DML-eig combined [103] & $85.65 \pm 0.56$ \\
\hline DDML (SSIFT: sqrt) & $\mathbf{8 7 . 8 3} \pm \mathbf{0 . 2 9}$ \\
\hline
\end{tabular}

Table 3.6: Comparison (\%) with state-of-the-art face verification methods on LFW under the image restricted setting, where NoD denotes the number of descriptors used.

\begin{tabular}{|l|c|c|}
\hline Method & NoD & Accuracy (\%) \\
\hline \hline CSML+SVM, aligned [8] & 6 & $88.00 \pm 0.37$ \\
PAF [72] & 1 & $87.77 \pm 0.51$ \\
STFRD+PMML [10] & 8 & $89.35 \pm 0.50$ \\
Sub-SML [104] & 6 & $89.73 \pm 0.38$ \\
VMRS [105] & 10 & $91.10 \pm 0.59$ \\
\hline DDML (HOG: sqrt) & 1 & $88.38 \pm 0.77$ \\
DDML (HDLBP: sqrt) & 1 & $\mathbf{9 2 . 5 5} \pm \mathbf{0 . 3 6}$ \\
\hline
\end{tabular}

state-of-the-art methods by $1.45 \%$ in terms of the mean verification rate under the image restricted paradigm, and $0.52 \%$ under the image unrestricted paradigm without using outside training data, respectively.

Comparison with Deep Learning Methods We compare the proposed DDML with several deep learning based face verification methods: CDBN [48], DNLML-ISA [22], ConvNet-RBM [108], DeepFace [24], and DeepID2 [26]. Table 3.8 shows the verification performance of different deep learning-based face verification methods on LFW. We see that our DDML outperforms the other deep learning methods without outside data. The 


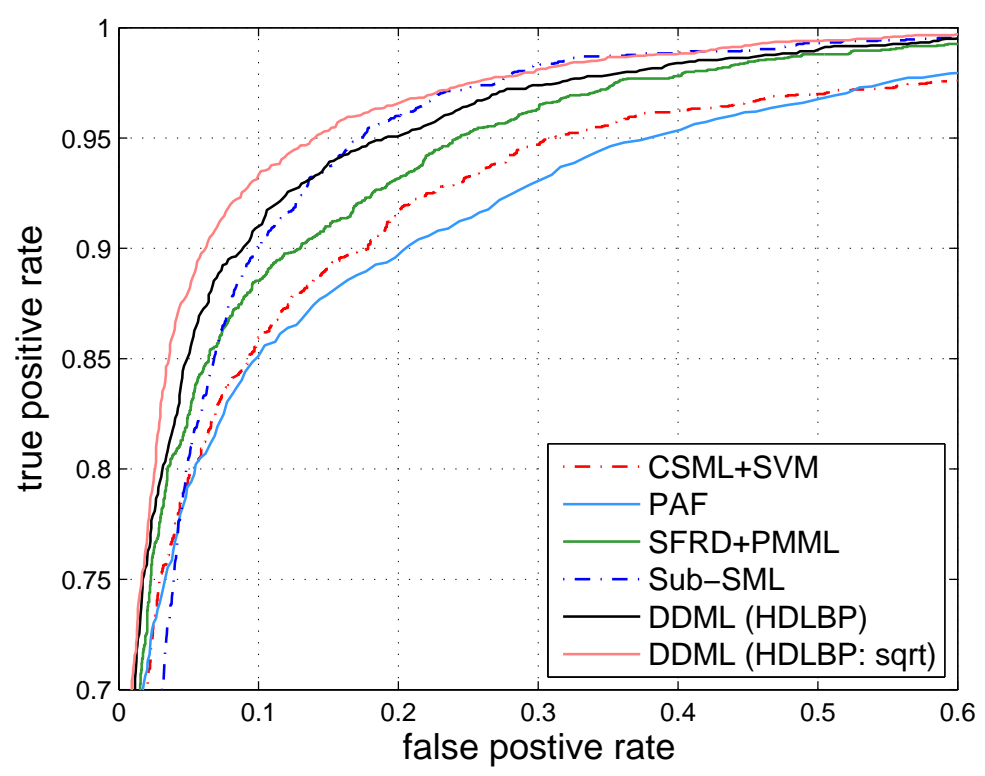

(a) Image restricted setting

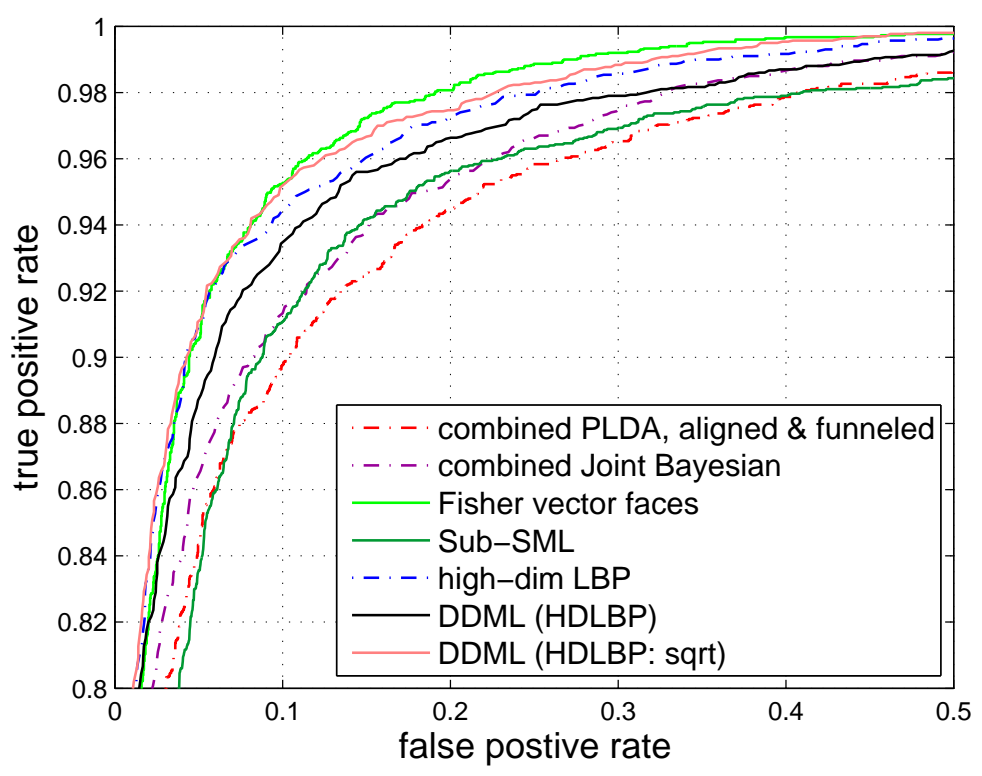

(b) Image unrestricted setting with label-free outside data

Figure 3.6: ROC comparison between our method and the state-of-the-art face verification methods on LFW under the (a) image restricted and (b) image unrestricted setting with label-free outside data, respectively. 
Table 3.7: Comparison with state-of-the-art results on LFW under the image unrestricted setting with label-free outside data, where NoD denotes the number of descriptors used in that method.

\begin{tabular}{|l|c|c|}
\hline Method & NoD & Accuracy (\%) \\
\hline \hline Combined PLDA [106] & 3 & $90.07 \pm 0.51$ \\
Combined Joint Bayesian [107] & 4 & $90.90 \pm 1.48$ \\
Sub-SML [104] & 6 & $90.75 \pm 0.64$ \\
VMRS [105] & 10 & $92.05 \pm 0.45$ \\
Fisher vector faces [73] & 1 & $93.03 \pm 1.05$ \\
High-dim LBP [100] & 1 & $93.18 \pm 1.07$ \\
\hline DDML (HDLBP: sqrt) & 1 & $\mathbf{9 3 . 7 0} \pm \mathbf{0 . 3 7}$ \\
\hline
\end{tabular}

reason is that CDBN is a unsupervised deep learning method and our DDML method is a supervised approach, so that more discriminative information can be exploited in our DDML method. Compared with DNLML-ISA which uses a stacked architecture, our DDML adopts a fully-connection architecture to design the network, which can explore better hierarchical information due to the back-propagation strategy. Compared with ConvNet-RBM, our method employs a large margin strategy to train the model, which is more effective than the RBM model used in ConvNet-RBM. Since there are $4.4 M$ outside face images used in DeepFace, DeepFace can learn more discriminative and robust features for face verification. Overall, our DDML achieves the state-of-the-art performance on LFW when no outside data is used on this dataset.

\section{Comparison with DeepFace and DeepID2 on LFW under Unrestricted Setting with}

Labeled Outside Data We also evaluated our DDML method using convolutional neural network $(\mathrm{CNN})$ feature for face verification [24, 26, 109]. In our implementations, we employed the VGG-Face CNN model provided by [109] to compute the CNN feature descriptor. Specifically, for each face image in the LFW dataset, we first cropped a $150 \times 150$ region from its center, and then resized it into $224 \times 224$ to compute a 4096-dimensional 
Table 3.8: Performance comparison (\%) with existing deep learning-based face verification methods on LFW.

\begin{tabular}{|l|c|c|c|}
\hline Method & Setting & Accuracy (\%) & Outside data \\
\hline \hline CDBN [48] & restricted & $86.88 \pm 0.62$ & No \\
CDBN+Hand-crafted [48] & restricted & $87.77 \pm 0.62$ & No \\
DNLML-ISA, combined [22] & unrestricted & $92.28 \pm 0.42$ & No \\
ConvNet-RBM [108] & unrestricted & 91.75 & No \\
ConvNet-RBM [108] & unrestricted & 92.52 & Yes ( $>87.6 K$ images) \\
DeepFace [24] & unrestricted & $97.25 \pm 0.81$ & Yes (4.4M images) \\
DeepID2 [26] & unrestricted & $\mathbf{9 9 . 1 5} \pm \mathbf{0 . 1 3}$ & Yes (0.2 $M$ images) \\
\hline DDML (HDLBP: sqrt) & restricted & $92.55 \pm 0.36$ & No \\
DDML (HDLBP: sqrt) & unrestricted & $93.70 \pm 0.37$ & No \\
\hline
\end{tabular}

Table 3.9: Comparison with DeepFace and DeepID2 on LFW dataset under the unrestricted setting with labeled outside data.

\begin{tabular}{|l|c|c|}
\hline Method & Feature & Accuracy (\%) \\
\hline \hline DDML & CNN & $98.23 \pm 0.22$ \\
\hline DeepFace [24] & CNN & $97.35 \pm 0.25$ \\
DeepID2 [26] & CNN & $\mathbf{9 9 . 1 5} \pm \mathbf{0 . 1 3}$ \\
\hline
\end{tabular}

CNN feature vector with the network. Moreover, each feature vector is reduced to the size of 300 by PCA for verification. Table 3.9 shows the mean verification accuracy and Figure 3.7 plots ROC curves of our method and the DeepFace and DeepID2 methods on the LFW dataset under the unrestricted setting with labeled outside data, respectively. We can see that 1) our proposed DDML is comparable to DeepFace [24], and 2) DeepID2 [26] obtains the best accuracy on the LFW dataset under the unrestricted setting with labeled outside data.

Comparison with Strongly-Supervised LMNN [4] Method Table 3.10 shows the performance comparison with LMNN [4] metric learning method on the LFW dataset under the image unrestricted setting with labeled outside data, where the SSIFT feature was used 


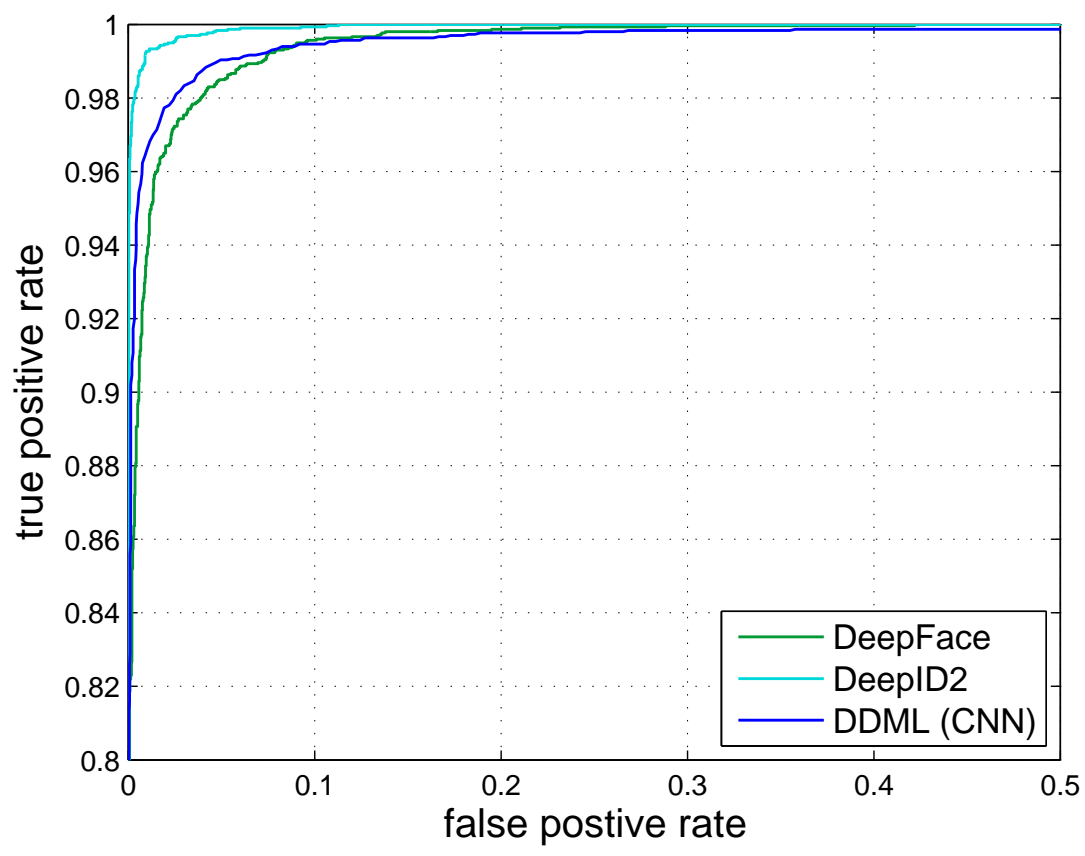

Figure 3.7: ROC curves of DDML, DeepFace and DeepID2 methods on LFW dataset under the unrestricted setting with labeled outside data.

Table 3.10: Comparisons (\%) with LMNN method on the LFW dataset under the image unrestricted setting with labeled outside data.

\begin{tabular}{|l|c|c|}
\hline Method & Setting & Accuracy \\
\hline \hline LMNN (SSIFT: sqrt) [7] & unrestricted & $80.50 \pm 0.50$ \\
\hline DDML (SSIFT: sqrt) & unrestricted & $88.92 \pm 0.45$ \\
\hline
\end{tabular}

for evaluation. We see that our DDML outperforms LMNN by $8.42 \%$ in terms of the mean verification rate.

Comparison with DNLML-ISA [22] Method under the Same Setting We compared the DDML method with DNLML-ISA [22] using the similar setting for a fair comparison, where the number of pair of each fold is set as 1000 for two methods. We used the sparse SIFT feature in our DDML method, which is the same as that used in [22]. Table 3.11 
Table 3.11: Comparison (\%) with DNLML-ISA method on the LFW dataset using the same setting.

\begin{tabular}{|l|c|c|c|}
\hline Method & SSIFT & SSIFT: sqrt & pairs per fold \\
\hline \hline DNLML-ISA & $85.14 \pm 0.39$ & $86.17 \pm 0.35$ & 1000 (unrestricted) \\
\hline DDML & $87.67 \pm 0.33$ & $88.36 \pm 0.41$ & 1000 (unrestricted) \\
\hline
\end{tabular}

Table 3.12: Comparison of the mean accuracy (\%) with the shallow metric learning methods on YTF under the image restricted setting.

\begin{tabular}{|l|c|c|}
\hline Feature & DDML & DSML \\
\hline \hline CSLBP & $75.98 \pm 0.89$ & $73.26 \pm 0.99$ \\
\hline FPLBP & $76.60 \pm 1.71$ & $73.46 \pm 1.66$ \\
\hline LBP & $81.26 \pm 1.63$ & $78.14 \pm 0.94$ \\
\hline
\end{tabular}

shows the results of our DDML method and the DNLML-ISA method on the LFW dataset. We can see that the DDML can obtain better performance than DNLML-ISA method.

Analysis of Failure Cases Figure 3.8 shows some example samples that were incorrectly verified by our DDML method on the LFW dataset under the image restricted setting, where "P" and "N" are ground truth labels. We can see that there are both large intra-class variations on positive pairs (e.g., pose, expression, and occlusion) and similar appearances on negative pairs, so that these image pairs are very challenging to face verification.

\subsubsection{Experimental Comparison on YTF}

Deep vs. Shallow Metric Learning We first compare our DDML with DSML method on the YTF dataset. Table 3.12 shows the verification rates with standard error when different feature descriptors are compared. Our DDML consistently outperforms DSML in terms of the mean verification rate on YTF dataset. 

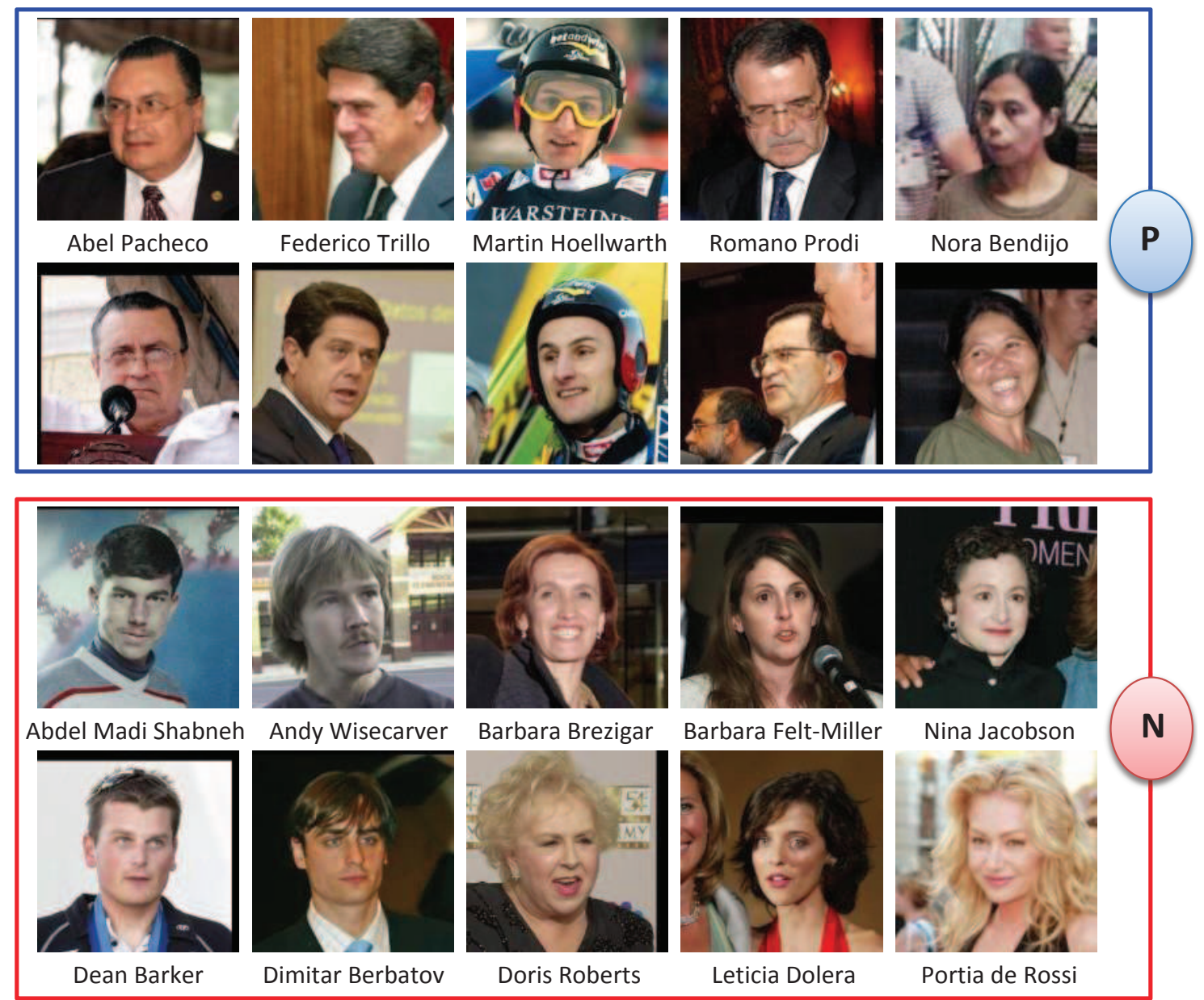

Figure 3.8: Some failure samples that were incorrectly verified by our DDML method on the LFW dataset under the image restricted setting, where $\mathbf{P}$ and $\mathbf{N}$ mean ground truth labels for positive and negative pairs, respectively.

Comparison with the State-of-the-Art methods We then compare our method with the state-of-the-art face verification methods on the YTF dataset ${ }^{5}$. These compared methods include Matched Background Similarity (MBGS) [74], Adaptive Probabilistic Elastic Matching (APEM) [110], STFRD+PMML [10], MBGS+SVM $\ominus$ [75], VSOF+OSS (Adaboost) [111], and PHL+SILD [102]. Table 3.13 and Figure 3.9 show the mean verification rate with the standard error and ROC curves of DDML and the state-of-the-art methods on the YTF dataset under image restricted setting, respectively. We see that the performance

\footnotetext{
${ }^{5}$ Available: http://www.cs.tau.ac.il/ wolf/ytfaces/results.html.
} 
Table 3.13: Comparison of the mean accuracy (\%) with the state-of-the-art face verification methods on YTF under the image restricted setting.

\begin{tabular}{|l|c|}
\hline Method & Accuracy (\%) \\
\hline \hline MBGS (LBP) [74] & $76.40 \pm 1.80$ \\
APEM (LBP) [110] & $77.44 \pm 1.46$ \\
APEM (fusion) [110] & $79.06 \pm 1.51$ \\
STFRD+PMML [10] & $79.48 \pm 2.52$ \\
MBGS+SVM $\ominus$ (LBP) [75] & $78.90 \pm 1.90$ \\
VSOF+OSS (Adaboost) [111] & $79.70 \pm 1.80$ \\
PHL+SILD (LBP) [102] & $80.20 \pm 1.30$ \\
\hline DDML (LBP) & $81.26 \pm 1.63$ \\
\hline
\end{tabular}

of our DDML with the LBP feature is $81.26 \pm 1.63$, which improves the PHL+SILD method by $1.0 \%$ in the gain of the mean verification rate.

\section{Comparison with DeepFace and DeepID2+ [112] on YTF under Image Unrestricted}

Setting Following the same VGG-Face CNN model [109] as used in the LFW dataset, we only extracted CNN features on the first 100 frames of each video at a single scale. For each face image in the YTF dataset, we first resized it to size of $200 \times 200$ pixels and cropped a $100 \times 100$ region from its center. Then, we resized it into $224 \times 224$ to compute a 4096-dimensional CNN feature vector. Finally, we average these CNN feature vectors of the first 100 frames for each video, and each video is represented by a 4096dimensional feature vector. Moreover, each feature vector is reduced to the size of 200 by PCA before verification. Table 3.14 shows the mean verification accuracy of our proposed DDML and several CNN based methods (e.g., DeepFace [24], DeepID2+ [112]) on the YTF dataset under the image unrestricted setting. In addition, Figure 3.10 plots ROC curves of several methods on the YTF dataset under the image unrestricted setting. We see that the performance of our DDML method are comparable to the current state-of-the-art results under the same setting. 


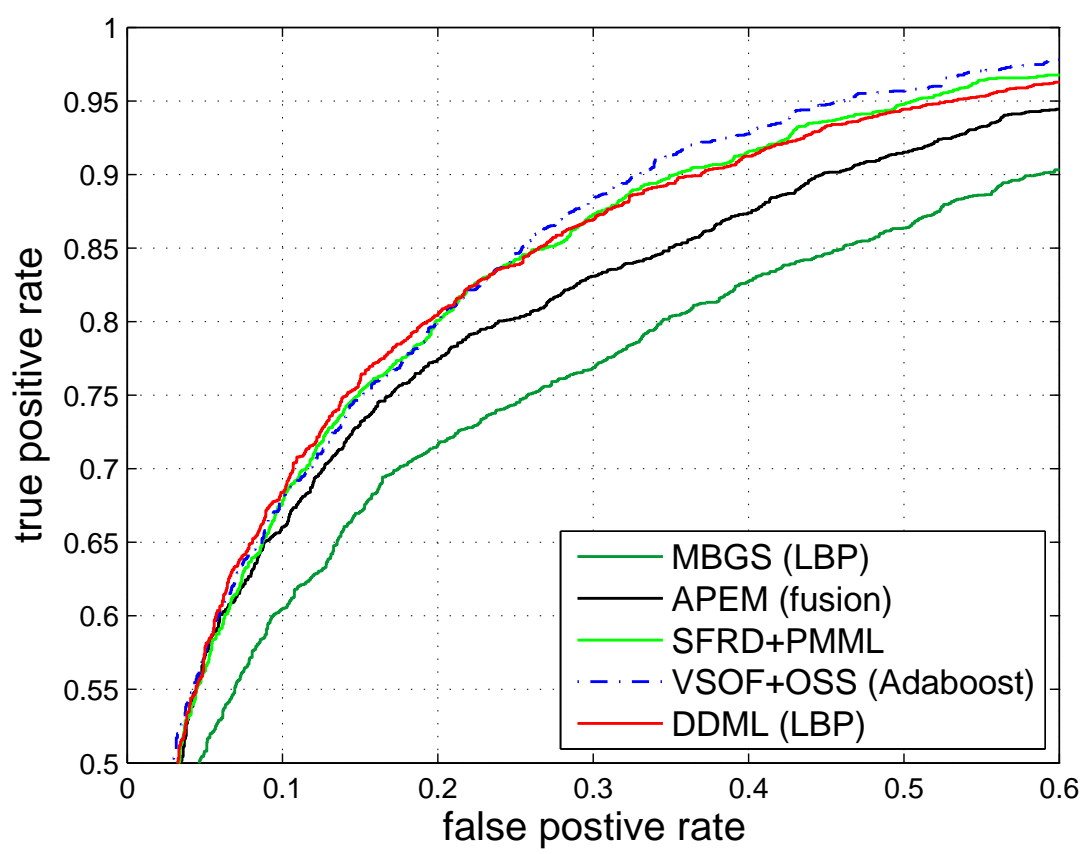

Figure 3.9: ROC curves comparison between our method and state-of-the-art face verification methods on YTF under image restricted setting.

Table 3.14: Comparison with CNN based methods on the YTF dataset under image unrestricted setting.

\begin{tabular}{|l|c|c|}
\hline Method & Feature & Accuracy (\%) \\
\hline \hline DDML & CNN & $94.58 \pm 1.01$ \\
\hline DeepFace [24] & CNN & $91.4 \pm 1.1$ \\
DeepID2+ [112] & CNN & $93.2 \pm 0.2$ \\
\hline
\end{tabular}

\subsubsection{Kinship Verification}

\subsubsection{Datasets and Experimental Settings}

The KinFaceW-I [83] and KinFaceW-II [83] are two kinship face datasets collected from the public figures or celebrities. For each person in these two datasets, face image of his/her parent or child was also collected. There are four kinship relations in these two datasets: F-S, F-D, M-S and M-D. In KinFaceW-I, there are 156, 134, 116, and 127 pairs of kinship images for these four relations. In KinFaceW-II, each relation contains 250 pairs of kinship 


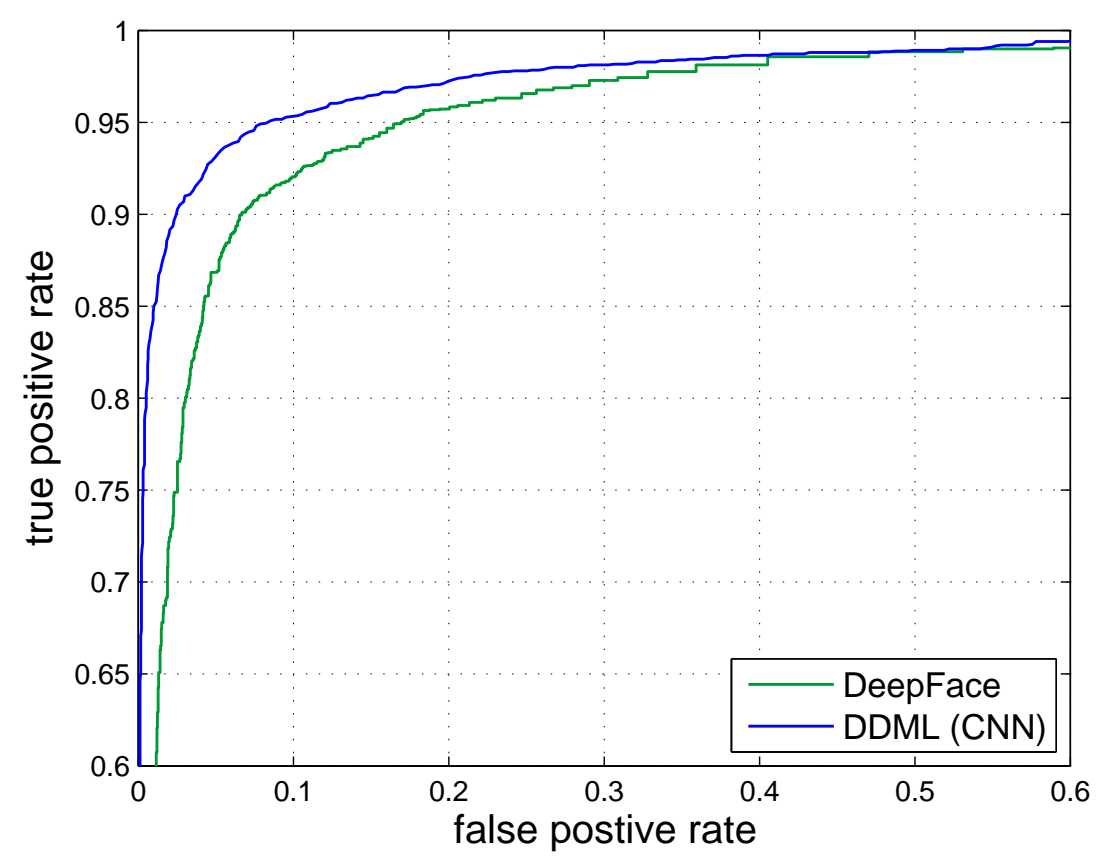

Figure 3.10: ROC curves of our method and DeepFace method on the YTF dataset under image unrestricted setting.

images. For these two datasets, we directly use each aligned $64 \times 64$ image for feature extraction. For each face image, we extract four feature descriptors: 1) LBP: we divide each image into $8 \times 8$ non-overlapping blocks, where the size of each block is $8 \times 8$. We extract a 59-dimensional uniform pattern LBP feature for each block and concatenate them to form a 3776-dimensional feature vector; 2) Dense SIFT (DSIFT): we densely sample SIFT descriptors on each $16 \times 16$ patch with stepsize of 8 pixels and obtain 49 SIFT descriptors. Then, we concatenate these SIFT descriptors to form a 6272-dimensional feature vector; 3) HOG: we first divide each image into $16 \times 16$ non-overlapping blocks, where the size of each block is $4 \times 4$. Then, we divide each image into $8 \times 8$ non-overlapping blocks, where the size of each block is $8 \times 8$. Subsequently, we extract a 9-dimensional HOG feature for each block and concatenate them to form a 2880-dimensional feature vector; and 4) Local phase quantization (LPQ) [113]: we first divide each image into $4 \times$ 
4 non-overlapping blocks, where the size of each block is $16 \times 16$. Then, we extract a 256-bin LPQ histogram with window size of 3, 5 and 7 for each block respectively, and finally concatenate them to form a 12288-dimensional feature vector. Following the same experimental settings in $[83,114]$, we adopt the five-fold cross validation strategy under the image restricted setting and the mean verification rate is used for the kinship verification performance evaluation. For parameters of our DDML, we use the same parameter settings as face verification, and Table 3.1 lists the size of $\left\{\mathbf{W}^{(m)}, \mathbf{b}^{(m)}\right\}_{m=1}^{M}$ for various features.

\subsubsection{Experimental Comparison on KinFaceW-I and KinFaceW-II}

Deep vs. Shallow Metric Learning Following the basic evaluation, we first compare our DDML method with the DSML method on the KinFaceW-I and KinFaceW-II datasets. Table 3.15 shows the mean verification rates of DDNL when different feature descriptors are used. As can be seen, our DDML consistently outperforms DSML in terms of the mean verification rate on both KinFaceW-I and KinFaceW-II datasets.

Comparison with the State-of-the-Art Methods Table 3.16 further shows the performance comparisons of our method with the state-of-the-art kinship verification methods. We see that the proposed DDML method achieves competitive performance compared with these state-of-the-art kinship verification methods.

\subsubsection{Effect of the Activation Function}

We analyze the effect of the activation function in our DDML method. We compare the tanh function with two other popular activation functions: sigmoid and non-saturating sigmoid (ns-sigmoid). Table 3.17 lists the performance of our DDML method with different activation functions on the LFW and YTF datasets, where the HDLBP (sqrt) and LBP features are used, respectively. We see that the tanh function performs the best and the sigmoid 


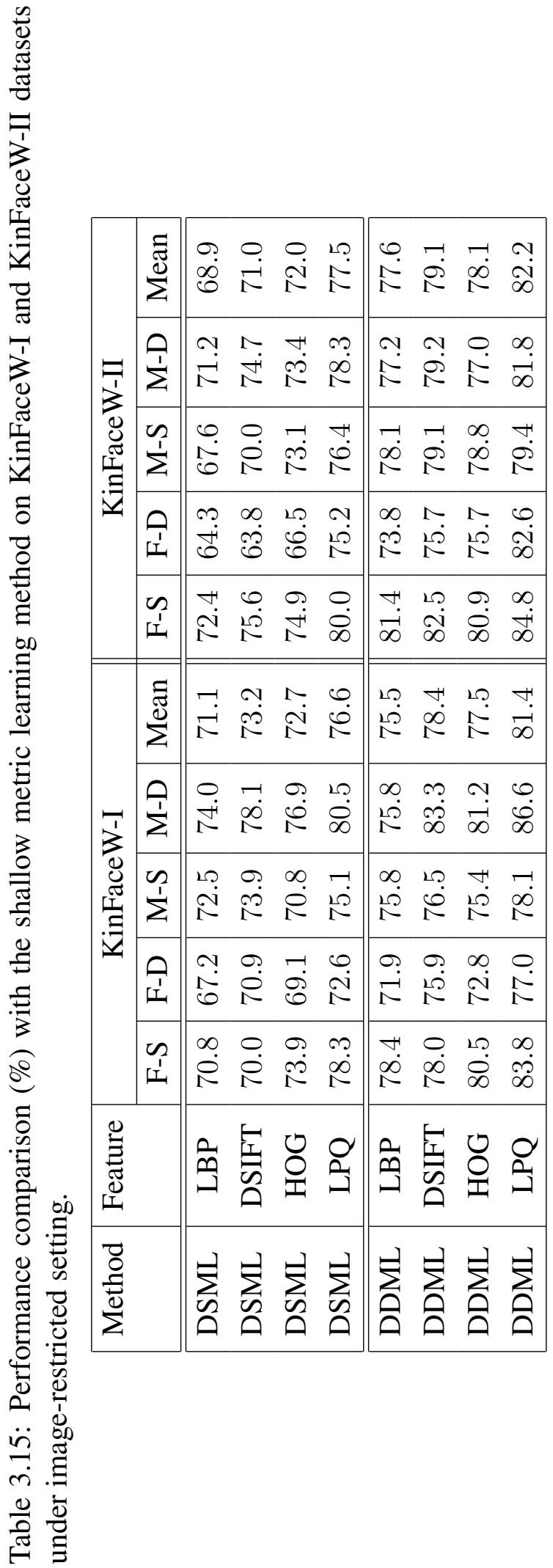


Table 3.16: Performance comparison (\%) with state-of-the-art methods on KinFaceW-I and KinFaceW-II datasets under image-restricted setting.

\begin{tabular}{|l|c|c|c|c|c||c|c|c|c|c|}
\hline \multirow{2}{*}{ Method } & \multicolumn{4}{|c||}{ KinFaceW-I } & \multicolumn{5}{c|}{ KinFaceW-II } \\
\cline { 2 - 11 } & F-S & F-D & M-S & M-D & Mean & F-S & F-D & M-S & M-D & Mean \\
\hline \hline SILD [18] & 80.5 & 72.4 & 69.8 & 77.1 & 74.9 & 79.6 & 71.6 & 73.2 & 69.6 & 73.5 \\
\hline ULPGC [114] & 71.3 & 70.9 & 58.5 & 80.9 & 70.0 & 85.4 & 75.8 & 75.6 & 81.6 & 80.0 \\
\hline BIU [114] & 86.9 & 76.5 & 73.9 & 79.8 & 79.3 & 87.5 & 80.8 & 79.8 & 75.6 & 80.9 \\
\hline \hline DDML & 83.8 & 77.0 & 78.1 & 86.6 & 81.4 & 84.8 & 82.6 & 79.4 & 81.8 & 82.2 \\
\hline
\end{tabular}

function performs the worst. The reason is that the output of the tanh activation function is symmetrical, where the output of the standard sigmoid activation function is asymmetrical. The asymmetry in the sigmoid function introduces a bias that pushes the activations of the later layers towards saturation, which is not a good position to obtain good training. For example, given a negative $x$ (e.g., -100$), \operatorname{sigmoid}(x)$ will lead to an activation 0 , however $\tanh (x)$ will lead to an activation -1 . Then, in the back-propagation step, sigmoid function cannot back-propagate anything, whereas the tanh function may back-propagate some useful information. Thus the gradients will be clearly different for the tanh and sigmoid activation functions. To some extent, this example can interpret the performance difference of the experimental results obtained by tanh and sigmoid functions.

Table 3.17: Comparison (\%) of different activation functions on LFW and YTF under the image restricted setting.

\begin{tabular}{|l|c|c|c|}
\hline Dataset & sigmoid & ns-sigmoid & tanh \\
\hline \hline LFW & $81.93 \pm 0.46$ & $92.10 \pm 0.43$ & $\mathbf{9 2 . 5 5} \pm \mathbf{0 . 3 6}$ \\
\hline YTF & $70.20 \pm 1.26$ & $80.78 \pm 1.15$ & $\mathbf{8 1 . 2 6} \pm \mathbf{1 . 6 3}$ \\
\hline
\end{tabular}

\subsubsection{Convergence Analysis}

We have evaluated the convergence of our DDML method. Figure 3.11 plots the value of the objective function of DDML versus different number of iterations on the LFW and YTF 
datasets under image restricted setting. In our experiments, the objective function values of DDML are the sum of all 5400 training pairs on LFW and 4500 pairs on YTF, respectively. We can see that the proposed DDML method converges in $30 \sim 40$ iterations on both LFW and YTF datasets.

\subsubsection{Computational Time}

Lastly, we report the computational time of DDML method on the LFW and YTF datasets under image restricted setting. In our implementations, we don't adopt parallel programming (1 core) and GPU acceleration, and we only run our MATLAB code on a PC whose hardware configuration comprises a 3.2-GHz CPU (Intel Core i5-3470) and a 8GB RAM. We use the SSIFT (sqrt) and LBP features for the DDML on the LFW and YTF datasets to compute the computational time. For each fold in these two datasets, the training times of DDML are 33.8 seconds and 27.4 seconds, and the test times are about 0.1 and 0.1 seconds on the LFW and YTF datasets, respectively. Our method is very fast, especially in training time, and it complements well to the existing deep learning methods.

\subsection{Conclusion}

In this chapter, we have presented a discriminative deep metric learning (DDML) method and applied DDML to face verification and kinship verification under the wild conditions. The DDML method can learn several hierarchical nonlinear transformations to map sample pairs into other feature subspace by using a neural network architecture, under which the distance of each positive sample pair is less than a small threshold and that of each negative pair is higher than a large threshold, so that discriminative information can be better exploited. Experimental results on face verification and kinship verification tasks show the 


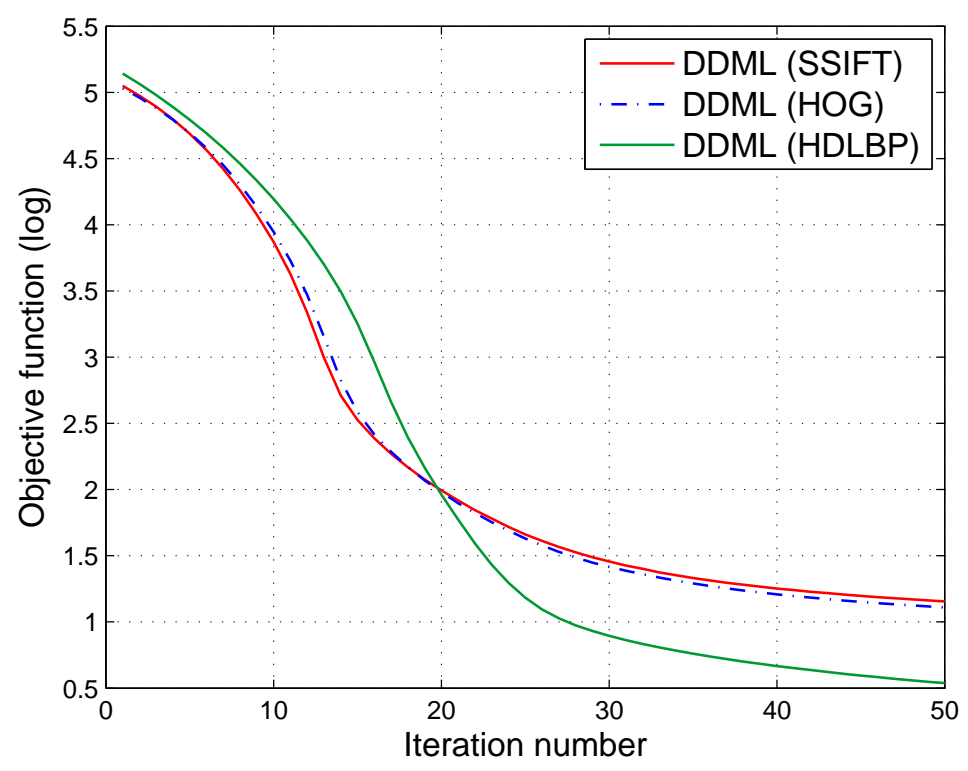

(a) LFW

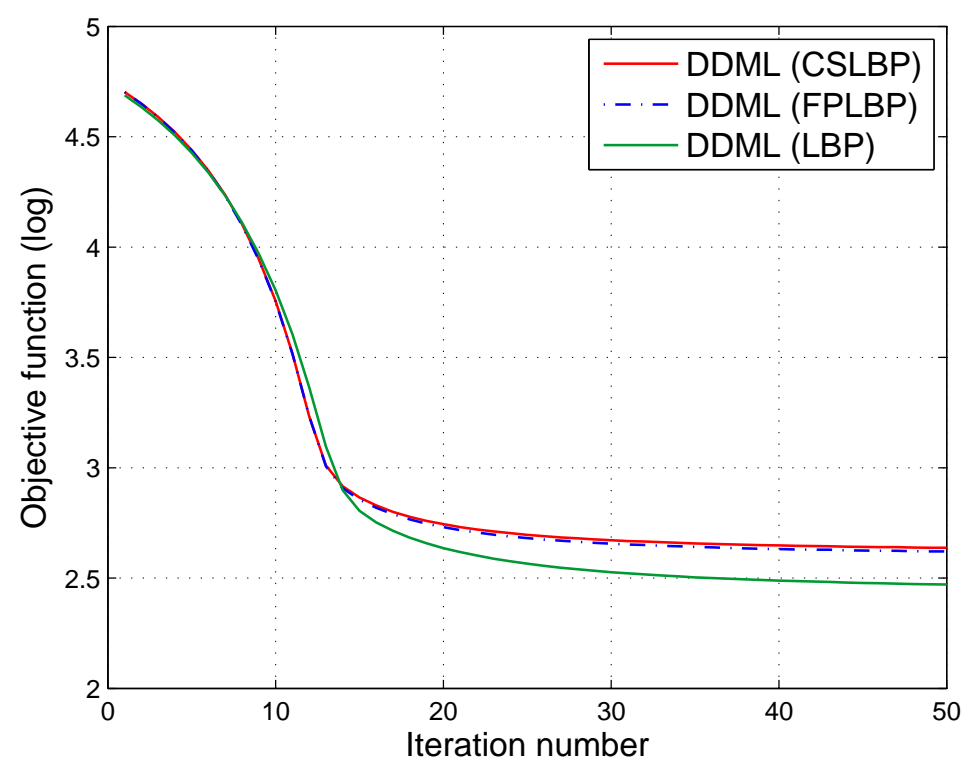

(b) YTF

Figure 3.11: Convergence curves of DDML on the (a) LFW and (b) YTF datasets under image restricted setting. 
effectiveness of the proposed method. The main part of the work presented in this chapter was published in $[11,96]$.

Most existing metric learning methods usually assume that the training and test samples are captured in similar scenarios so that their distributions are assumed to be the same. This assumption doesn't hold in many real visual recognition applications, especially when samples are captured across different datasets, environments, sessions, etc. In addition, how to learn a suitable distance metric on the training datasets which consist of labeled source datasets and unlabeled target dataset, and fitting the metric to the unlabeled target dataset is another challenging problem in metric learning. To address this, in the following chapter, we present a deep transfer metric learning (DTML) method to learn a set of hierarchical nonlinear transformations for cross-domain visual recognition by transferring discriminative knowledge from the labeled source domain to the unlabeled target domain.

\section{Appendix: KDSML}

Kernel Discriminative Shallow Metric Learning (KDSML) employs the kernel trick to implicitly map samples into a high-dimensional feature space and then performs discriminative shallow metric learning (DSML) in that space to learn a distance metric. We assume there is a mapping $\phi: \mathbb{R} \rightarrow \mathbb{F}$ which maps each sample $\mathrm{x}$ into a high-dimensional space $\mathbb{F}$ as $\phi(\mathbf{x})$. The squared Mahalanobis distance between two data points $\mathbf{x}_{i}$ and $\mathbf{x}_{j}$ in that space is computed as:

$$
\begin{aligned}
d_{\mathbf{W}}^{2}\left(\mathbf{x}_{i}, \mathbf{x}_{j}\right) & =\left(\phi\left(\mathbf{x}_{i}\right)-\phi\left(\mathbf{x}_{j}\right)\right)^{T} \mathbf{M}\left(\phi\left(\mathbf{x}_{i}\right)-\phi\left(\mathbf{x}_{j}\right)\right) \\
& =\left(\phi\left(\mathbf{x}_{i}\right)-\phi\left(\mathbf{x}_{j}\right)\right)^{T} \mathbf{W}^{T} \mathbf{W}\left(\phi\left(\mathbf{x}_{i}\right)-\phi\left(\mathbf{x}_{j}\right)\right) \\
& =\left\|\mathbf{W} \phi\left(\mathbf{x}_{i}\right)-\mathbf{W} \phi\left(\mathbf{x}_{j}\right)\right\|_{2}^{2}
\end{aligned}
$$


where $\mathbf{M}$ is the learned Mahalanobis distance metric, $\mathbf{W}$ is a transformation matrix, and we have $\mathbf{M}=\mathbf{W}^{T} \mathbf{W}$.

Given a kernel function $\kappa\left(\mathbf{x}_{i}, \mathbf{x}_{j}\right)=\phi\left(\mathbf{x}_{i}\right)^{T} \phi\left(\mathbf{x}_{j}\right)$, we then define $\Phi=\left[\phi\left(\mathbf{x}_{1}\right), \phi\left(\mathbf{x}_{2}\right), \cdots\right.$, $\left.\phi\left(\mathbf{x}_{N}\right)\right]^{T}$ and $\mathbf{k}_{i}=\Phi \phi\left(\mathbf{x}_{i}\right)=\left[\kappa\left(\mathbf{x}_{1}, \mathbf{x}_{i}\right), \kappa\left(\mathbf{x}_{2}, \mathbf{x}_{i}\right), \cdots, \kappa\left(\mathbf{x}_{N}, \mathbf{x}_{i}\right)\right]^{T}$. We parameterize $\mathbf{W}$ as the form $\mathbf{W}=\mathbf{A} \Phi$ by following [115], where $\mathbf{A}$ is a coefficient matrix and each row of W is a linear combination of samples in $\Phi$. Then, distance (3.21) can be rewritten as:

$$
\begin{aligned}
d_{\mathbf{W}}^{2}\left(\mathbf{x}_{i}, \mathbf{x}_{j}\right) & =\left\|\mathbf{W} \phi\left(\mathbf{x}_{i}\right)-\mathbf{W} \phi\left(\mathbf{x}_{j}\right)\right\|_{2}^{2} \\
& =\left\|\mathbf{A} \Phi \phi\left(\mathbf{x}_{i}\right)-\mathbf{A} \Phi \phi\left(\mathbf{x}_{j}\right)\right\|_{2}^{2} \\
& =\left\|\mathbf{A} \mathbf{k}_{i}-\mathbf{A} \mathbf{k}_{j}\right\|_{2}^{2}=\left\|\mathbf{A}\left(\mathbf{k}_{i}-\mathbf{k}_{j}\right)\right\|_{2}^{2} \\
& =d_{\mathbf{A}}^{2}\left(\mathbf{x}_{i}, \mathbf{x}_{j}\right) .
\end{aligned}
$$

The objective function of KDSML is then formulated as:

$$
\arg \min _{\mathbf{A}} J=\frac{1}{2} \sum_{i, j} g\left(1-\ell_{i j}\left(\tau-d_{\mathbf{A}}^{2}\left(\mathbf{x}_{i}, \mathbf{x}_{j}\right)\right)\right)+\frac{\lambda}{2}\|\mathbf{A}\|_{F}^{2},
$$

where $g(z)=\frac{1}{\beta} \log (1+\exp (\beta z))$ is the generalized logistic loss function, which is a smoothed approximation of the hinge loss function $[z]_{+}=\max (z, 0) ; \beta$ is a sharpness parameter; $\|\mathbf{A}\|_{F}$ represents the Frobenius norm of the matrix $\mathbf{A} ; \lambda$ is a regularization parameter; and $\ell_{i j}$ is the pairwise label between $\mathbf{x}_{i}$ and $\mathbf{x}_{j}$. There are two terms in the objective function (3.23), where the first term defines the logistic loss of training samples and the second term represents the regularizer of the coefficient matrix $\mathbf{A}$.

To solve the optimization problem in (3.23), we use the gradient descent based method to obtain $\mathbf{A}$. The derivative of the objective function $J$ with respect to $\mathbf{A}$ is computed as:

$$
\frac{\partial J}{\partial \mathbf{A}}=\mathbf{A} \sum_{i, j} g^{\prime}(c) \ell_{i j}\left(\mathbf{k}_{i}-\mathbf{k}_{j}\right)\left(\mathbf{k}_{i}-\mathbf{k}_{j}\right)^{T}+\lambda \mathbf{A},
$$

where $c=1-\ell_{i j}\left(\tau-d_{\mathbf{A}}^{2}\left(\mathbf{x}_{i}, \mathbf{x}_{j}\right)\right)$. In our experiments, we used the RBF kernel $\kappa\left(\mathbf{x}_{i}, \mathbf{x}_{j}\right)=$ $\exp \left(-\gamma\left\|\mathbf{x}_{i}-\mathbf{x}_{j}\right\|_{2}^{2}\right)$. 


\section{Chapter 4}

\section{Deep Transfer Metric Learning}

In the previous chapter, we have proposed a discriminative deep metric learning (DDML) method to address the nonlinearity issue of metric learning. Most existing metric learning methods usually assume that the training and test samples are captured in similar scenarios and their distributions are the same or similar. This assumption doesn't hold in many real-world visual applications, especially when samples are captured across different datasets. To address this problem, we propose in this chapter a deep transfer metric learning (DTML) method, and further develop a deeply supervised transfer metric learning (DSTML) method. To preserve the local manifold of data points, we also present DTML with autoencoder regularization (DTML-AE) and DSTML with autoencoder regularization (DSTML-AE) methods. Experimental results on face verification, person re-identification and handwritten digit recognition validate the effectiveness of the proposed methods.

\subsection{Introduction}

In recent years, a variety of metric learning algorithms have been proposed in the literature $[3,4,68,117]$, and some of them have successfully been applied to visual analysis applications such as face recognition [11, 118, 119], image classification [3, 4, 120], and

The key part of the work presented in this chapter was published in $[29,116]$. 
visual tracking [35]. Existing metric learning methods can be mainly classified into two categories: unsupervised and supervised. For the first category, a low-dimensional subspace or manifold is learned to preserve the geometrical information of the samples. For the second category, a discriminative distance metric is learned to maximize the separability of samples from different classes. Since the label information of training samples is used, supervised metric learning methods are more suitable for the recognition task.

While many supervised metric learning algorithms have been proposed in recent years, there are still two shortcomings of these methods: 1) most of them usually seek a single linear distance to transform sample into a linear feature space, so that the nonlinear relationship of samples cannot be well exploited. Even if the kernel trick [15] can be employed to address the nonlinearity issue, these methods still suffer from the scalability problem because they cannot obtain the explicit nonlinear mapping functions; 2) most of them assume that the training and test samples are captured in similar scenarios so that their distributions are assumed to be the same. This assumption doesn't hold in many real visual recognition applications, especially when samples are captured across different datasets.

To this end, we propose in this chapter a new deep transfer metric learning (DTML) method for cross-domain visual recognition. Figure 4.1 illustrates the basic idea of the proposed method. Our method learns a set of hierarchical nonlinear transformations by transferring discriminative knowledge from the labeled source domain to the unlabeled target domain, under which the inter-class variations are maximized and the intra-class variations are minimized, and the distribution divergence between the source domain and the target domain at the top layer of the network is minimized, simultaneously. To better exploit the discriminative information from the source domain, we further develop a deeply supervised transfer metric learning (DSTML) method by including an additional objective on DTML where the output of both the hidden layers and the top layer are optimized jointly. Furthermore, to capture the local manifold structure of input data points 


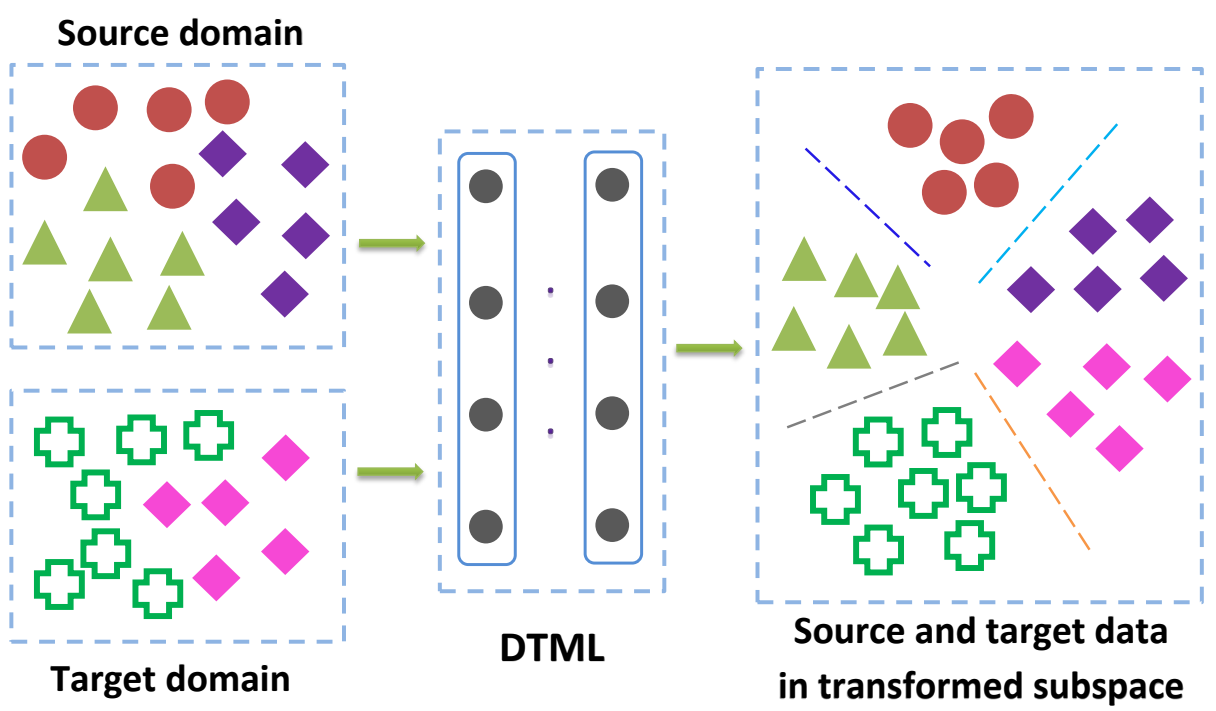

Figure 4.1: The basic idea of the DTML method. For each sample in the training sets from the source domain and the target domain, we pass it to the developed deep neural network. We enforce two constraints on the outputs of all training samples at the top of the network: 1) the inter-class variations are maximized and the intra-class variations are minimized, and 2) the distribution divergence between the source domain and the target domain at the top layer of the network is minimized.

as well as easily recover resulting data points in the metric space to their original forms, we employ the autoencoder as a complementary regularizer to regularize our DTML and DSTML methods and further introduce two new methods, deep transfer metric learning with autoencoder regularization (DTML-AE) and deeply supervised transfer metric learning with autoencoder regularization (DSTML-AE), respectively. Experimental results on three cross-domain visual recognition tasks demonstrate the effectiveness of the proposed methods.

\subsection{Deep Transfer Metric Learning}

In this section, we first introduce the notation used in this work. Then, we present the deep metric learning framework, the proposed deep transfer metric learning method and deeply supervised transfer metric learning method. 


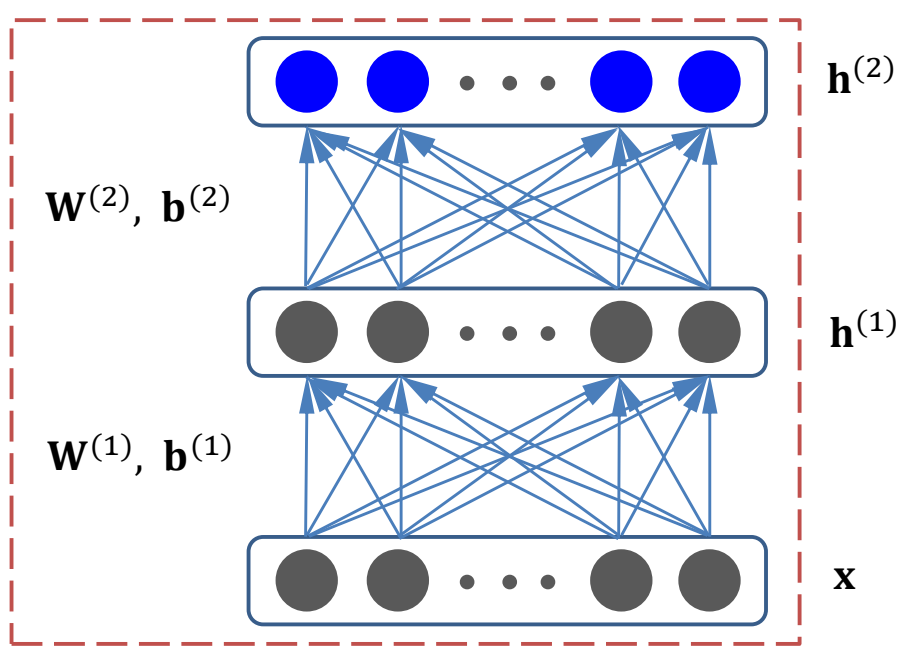

Figure 4.2: The network architecture used in our method. The input to the network is $\mathbf{x}$, and the output of the hidden layer and the top layer is $\mathbf{h}^{(1)}$ and $\mathbf{h}^{(2)}$, respectively. Here $\mathbf{W}^{(m)}$ and $\mathbf{b}^{(m)}$ are the parameters of the network to be learned, $1 \leq m \leq 2$.

\subsubsection{Notation}

Let $\mathcal{X}_{s}=\left\{\left(\mathbf{x}_{s i}, y_{s i}\right) \mid i=1,2, \cdots, N_{s}\right\}$ be the training set in the source domain, which contains $N_{s}$ examples, where $\mathbf{x}_{s i} \in \mathbb{R}^{d}$ is a $d$-dimensional feature vector, $y_{s i} \in\left\{1,2, \cdots, C_{s}\right\}$ is the label of $\mathbf{x}_{s i}$, and $C_{s}$ is the number of classes. Similarly, we denote $\mathcal{X}_{t}=\left\{\mathbf{x}_{t i} \mid i=\right.$ $\left.1,2, \cdots, N_{t}\right\}$ be the training samples in the target domain, where $N_{t}$ is the number of samples. Let $\mathcal{X}=\left\{\left(\mathbf{x}_{i}, y_{i}\right) \mid i=1,2, \cdots, N\right\}$ be the labeled training set. In our experiments, the labeled training set $\mathcal{X}$ is only sampled from the source domain $\mathcal{X}_{s}$ (i.e., $\mathcal{X}=\mathcal{X}_{s}$ ). Note that the labels $y_{s i}$ of training samples in source domain are used to compute the intra-class compactness and the interclass separability.

\subsubsection{Deep Metric Learning}

Unlike most previous metric learning methods which usually seek a single linear distance to transform sample into a linear feature space, we construct a feed-forward neural network to compute the representations of a sample $\mathrm{x}$ by passing it to multiple layers of nonlinear transformations, as shown in Figure 4.2. The key advantage of using such a network to map 
$\mathbf{x}$ is that the nonlinear mapping function can be explicitly obtained. Assume there are $M+1$ layers in the designed network and $p^{(m)}$ units in the $m$ th layer, where $m=1,2, \cdots, M$. The output of $\mathbf{x}$ at the $m$ th layer is computed as:

$$
f^{(m)}(\mathbf{x})=\mathbf{h}^{(m)}=\varphi\left(\mathbf{W}^{(m)} \mathbf{h}^{(m-1)}+\mathbf{b}^{(m)}\right)
$$

where $\mathbf{W}^{(m)} \in \mathbb{R}^{p^{(m)} \times p^{(m-1)}}$ and $\mathbf{b}^{(m)} \in \mathbb{R}^{p^{(m)}}$ are the weight matrix and bias of the parameters in this layer; and $\varphi$ is a nonlinear activation function which operates componentwisely, such as widely used tanh or sigmoid functions. The nonlinear mapping $f^{(m)}: \mathbb{R}^{d} \mapsto$ $\mathbb{R}^{p^{(m)}}$ is a function parameterized by $\left\{\mathbf{W}^{(i)}\right\}_{i=1}^{m}$ and $\left\{\mathbf{b}^{(i)}\right\}_{i=1}^{m}$. For the first layer, we assume $\mathbf{h}^{(0)}=\mathbf{x}$ and $p^{(0)}=d$.

For each pair of samples $\mathbf{x}_{i}$ and $\mathbf{x}_{j}$, they can be finally represented as $f^{(m)}\left(\mathbf{x}_{i}\right)$ and $f^{(m)}\left(\mathbf{x}_{j}\right)$ at the $m$ th layer of our designed network, and their distance metric can be measured by computing the squared Euclidean distance between the representations $f^{(m)}\left(\mathbf{x}_{i}\right)$ and $f^{(m)}\left(\mathbf{x}_{j}\right)$ at the $m$ th layer:

$$
d_{f^{(m)}}^{2}\left(\mathbf{x}_{i}, \mathbf{x}_{j}\right)=\left\|f^{(m)}\left(\mathbf{x}_{i}\right)-f^{(m)}\left(\mathbf{x}_{j}\right)\right\|_{2}^{2}
$$

Following the graph embedding framework, we enforce the marginal fisher analysis criterion [121] on the output of all the training samples at the top layer and formulate a strongly-supervised deep metric learning method as:

$$
\min _{f^{(M)}} J=S_{c}^{(M)}-\alpha S_{b}^{(M)}+\gamma \sum_{m=1}^{M}\left(\left\|\mathbf{W}^{(m)}\right\|_{F}^{2}+\left\|\mathbf{b}^{(m)}\right\|_{2}^{2}\right),
$$

where $\alpha(\alpha>0)$ is a free parameter which balances the importance between intra-class compactness and interclass separability; $\|\mathbf{Z}\|_{F}$ denotes the Frobenius norm of the matrix $\mathbf{Z} ; \gamma(\gamma>0)$ is a tunable positive regularization parameter; $S_{c}^{(m)}$ and $S_{b}^{(m)}$ define the intra- 
class compactness and the interclass separability, which are defined as follows:

$$
\begin{aligned}
S_{c}^{(m)} & =\frac{1}{N k_{1}} \sum_{i=1}^{N} \sum_{j=1}^{N} P_{i j} d_{f^{(m)}}^{2}\left(\mathbf{x}_{i}, \mathbf{x}_{j}\right), \\
S_{b}^{(m)} & =\frac{1}{N k_{2}} \sum_{i=1}^{N} \sum_{j=1}^{N} Q_{i j} d_{f^{(m)}}^{2}\left(\mathbf{x}_{i}, \mathbf{x}_{j}\right),
\end{aligned}
$$

where $P_{i j}=1$ if $\mathbf{x}_{j}$ is one of $k_{1}$-intraclass nearest neighbors of $\mathbf{x}_{i}$, and 0 otherwise; $Q_{i j}=1$ if $\mathbf{x}_{j}$ is one of $k_{2}$-interclass nearest neighbors of $\mathbf{x}_{i}$, and 0 otherwise; and $k_{1}$ and $k_{2}$ are two positive integers.

\subsubsection{Deep Transfer Metric Learning}

Given target domain data $\mathcal{X}_{t}$ and source domain data $\mathcal{X}_{s}$, their probability distributions are usually different in the original feature space when they are captured from different datasets. To reduce the distribution difference, it is desirable to make the probability distribution of the source domain and that of the target domain be as close as possible in the transformed space. To achieve this, we apply the Maximum Mean Discrepancy (MMD) criterion [59] to measure their distribution difference at the $m$ th layer, which is defined as:

$$
D_{t s}^{(m)}\left(\mathcal{X}_{t}, \mathcal{X}_{s}\right)=\left\|\frac{1}{N_{t}} \sum_{i=1}^{N_{t}} f^{(m)}\left(\mathbf{x}_{t i}\right)-\frac{1}{N_{s}} \sum_{i=1}^{N_{s}} f^{(m)}\left(\mathbf{x}_{s i}\right)\right\|_{2}^{2}
$$

By combining (4.3) and (4.6), we formulate DTML as the following optimization problem:

$$
\min _{f^{(M)}} J=S_{c}^{(M)}-\alpha S_{b}^{(M)}+\beta D_{t s}^{(M)}\left(\mathcal{X}_{t}, \mathcal{X}_{s}\right)+\gamma \sum_{m=1}^{M}\left(\left\|\mathbf{W}^{(m)}\right\|_{F}^{2}+\left\|\mathbf{b}^{(m)}\right\|_{2}^{2}\right),
$$

where $\beta(\beta \geq 0)$ is a regularization parameter.

To solve the optimization problem in (4.7), we employ the stochastic sub-gradient descent method to obtain the parameters $\mathbf{W}^{(m)}$ and $\mathbf{b}^{(m)}$. The gradients of the objective 
function $J$ in (4.7) with respect to the parameters $\mathbf{W}^{(m)}$ and $\mathbf{b}^{(m)}$ are computed as follows:

$$
\begin{aligned}
\frac{\partial J}{\partial \mathbf{W}^{(m)}} & =\frac{2}{N k_{1}} \sum_{i=1}^{N} \sum_{j=1}^{N} P_{i j}\left(\mathbf{L}_{i j}^{(m)} \mathbf{h}_{i}^{(m-1)^{T}}+\mathbf{L}_{j i}^{(m)} \mathbf{h}_{j}^{(m-1)^{T}}\right) \\
& -\frac{2 \alpha}{N k_{2}} \sum_{i=1}^{N} \sum_{j=1}^{N} Q_{i j}\left(\mathbf{L}_{i j}^{(m)} \mathbf{h}_{i}^{(m-1)^{T}}+\mathbf{L}_{j i}^{(m)} \mathbf{h}_{j}^{(m-1)^{T}}\right) \\
& +2 \beta\left(\frac{1}{N_{t}} \sum_{i=1}^{N_{t}} \mathbf{L}_{t i}^{(m)} \mathbf{h}_{t i}^{(m-1)^{T}}+\frac{1}{N_{s}} \sum_{i=1}^{N_{s}} \mathbf{L}_{s i}^{(m)} \mathbf{h}_{s i}^{(m-1)^{T}}\right)+2 \gamma \mathbf{W}^{(m)} \\
\frac{\partial J}{\partial \mathbf{b}^{(m)}} & =\frac{2}{N k_{1}} \sum_{i=1}^{N} \sum_{j=1}^{N} P_{i j}\left(\mathbf{L}_{i j}^{(m)}+\mathbf{L}_{j i}^{(m)}\right) \\
& -\frac{2 \alpha}{N k_{2}} \sum_{i=1}^{N} \sum_{j=1}^{N} Q_{i j}\left(\mathbf{L}_{i j}^{(m)}+\mathbf{L}_{j i}^{(m)}\right) \\
& +2 \beta\left(\frac{1}{N_{t}} \sum_{i=1}^{N_{t}} \mathbf{L}_{t i}^{(m)}+\frac{1}{N_{s}} \sum_{i=1}^{N_{s}} \mathbf{L}_{s i}^{(m)}\right)+2 \gamma \mathbf{b}^{(m)}
\end{aligned}
$$

where the updating equations are computed as follows:

$$
\begin{aligned}
& \mathbf{L}_{i j}^{(M)}=\left(\mathbf{h}_{i}^{(M)}-\mathbf{h}_{j}^{(M)}\right) \odot \varphi^{\prime}\left(\mathbf{z}_{i}^{(M)}\right), \\
& \mathbf{L}_{j i}^{(M)}=\left(\mathbf{h}_{j}^{(M)}-\mathbf{h}_{i}^{(M)}\right) \odot \varphi^{\prime}\left(\mathbf{z}_{j}^{(M)}\right), \\
& \mathbf{L}_{i j}^{(m)}=\left(\mathbf{W}^{(m+1)^{T}} \mathbf{L}_{i j}^{(m+1)}\right) \odot \varphi^{\prime}\left(\mathbf{z}_{i}^{(m)}\right), \\
& \mathbf{L}_{j i}^{(m)}=\left(\mathbf{W}^{(m+1)^{T}} \mathbf{L}_{j i}^{(m+1)}\right) \odot \varphi^{\prime}\left(\mathbf{z}_{j}^{(m)}\right), \\
& \mathbf{L}_{t i}^{(M)}=\left(\frac{1}{N_{t}} \sum_{j=1}^{N_{t}} \mathbf{h}_{t j}^{(M)}-\frac{1}{N_{s}} \sum_{j=1}^{N_{s}} \mathbf{h}_{s j}^{(M)}\right) \odot \varphi^{\prime}\left(\mathbf{z}_{t i}^{(M)}\right), \\
& \mathbf{L}_{s i}^{(M)}=\left(\frac{1}{N_{s}} \sum_{j=1}^{N_{s}} \mathbf{h}_{s j}^{(M)}-\frac{1}{N_{t}} \sum_{j=1}^{N_{t}} \mathbf{h}_{t j}^{(M)}\right) \odot \varphi^{\prime}\left(\mathbf{z}_{s i}^{(M)}\right), \\
& \mathbf{L}_{t i}^{(m)}=\left(\mathbf{W}^{(m+1)}{ }^{T} \mathbf{L}_{t i}^{(m+1)}\right) \odot \varphi^{\prime}\left(\mathbf{z}_{t i}^{(m)}\right), \\
& \mathbf{L}_{s i}^{(m)}=\left(\mathbf{W}^{(m+1)} \mathbf{L}_{s i}^{(m+1)}\right) \odot \varphi^{\prime}\left(\mathbf{z}_{s i}^{(m)}\right),
\end{aligned}
$$

where $m=1,2, \cdots, M-1$. Here the operation $\odot$ denotes the element-wise multiplication, and $\mathbf{z}_{i}^{(m)}$ is given as $\mathbf{z}_{i}^{(m)}=\mathbf{W}^{(m)} \mathbf{h}_{i}^{(m-1)}+\mathbf{b}^{(m)}$. 


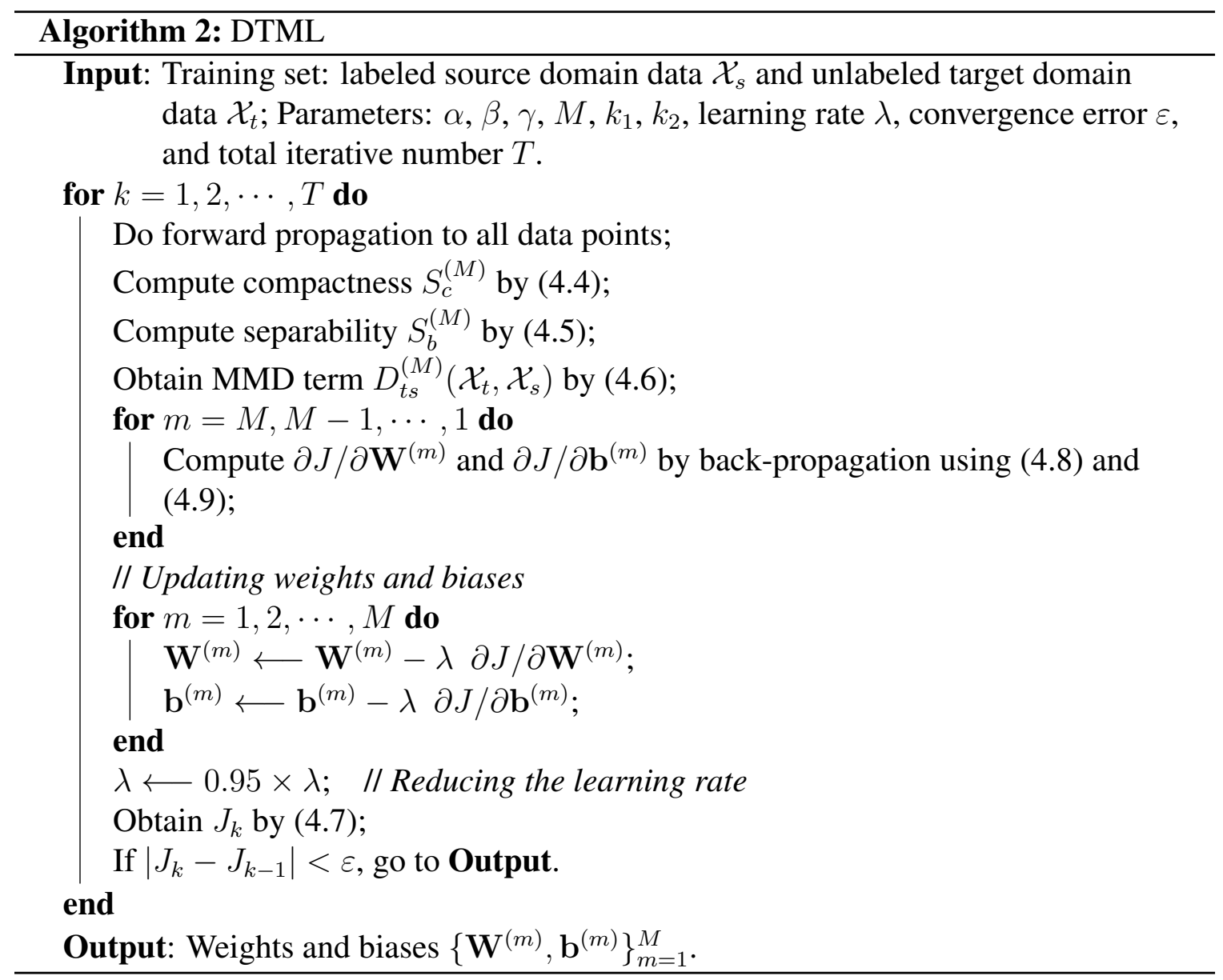

Then, $\mathbf{W}^{(m)}$ and $\mathbf{b}^{(m)}$ can be updated by using the gradient descent algorithm as follows until convergence:

$$
\begin{aligned}
\mathbf{W}^{(m)} & =\mathbf{W}^{(m)}-\lambda \frac{\partial J}{\partial \mathbf{W}^{(m)}}, \\
\mathbf{b}^{(m)} & =\mathbf{b}^{(m)}-\lambda \frac{\partial J}{\partial \mathbf{b}^{(m)}},
\end{aligned}
$$

where $\lambda$ is the learning rate.

Algorithm 2 summarizes the detailed optimization procedure of the proposed DTML.

\subsubsection{Deeply Supervised Transfer Metric Learning}

The objective function of DTML defined in (4.7) only considers the supervised information of training samples at the top layer of the network, which ignore the discriminative 
information of the output at the hidden layers. To address this, we further propose a deeply supervised transfer metric learning (DSTML) method to better exploit discriminative information from the output of all layers. We formulate the following optimization problem:

$$
\min _{f^{(M)}} J=J^{(M)}+\sum_{m=1}^{M-1} \omega^{(m)} h\left(J^{(m)}-\tau^{(m)}\right),
$$

where

$$
J^{(m)}=S_{c}^{(m)}-\alpha S_{b}^{(m)}+\beta D_{t s}^{(m)}\left(\mathcal{X}_{t}, \mathcal{X}_{s}\right)+\gamma\left(\left\|\mathbf{W}^{(m)}\right\|_{F}^{2}+\left\|\mathbf{b}^{(m)}\right\|_{2}^{2}\right)
$$

is the objective function of DTML applied at the $m$ th layer. Here $J^{(M)}$ is the loss of the top layer and $J^{(m)}$ is the loss of the $m$ th hidden layer, $m=1,2, \cdots, M-1$. The hinge loss function $h(x)=\max (x, 0)$ is used to measure the loss; $\tau^{(m)}$ is a positive threshold, which controls the loss $J^{(m)}$ to show it plays the role in the learning procedure; and $\omega^{(m)}$ balances the importance of the loss obtained at the top layer and the $m$ th hidden layer. The second term in (4.12) will disappear during the learning procedure if the overall loss of the $m$ th hidden layer is below the threshold $\tau^{(m)}$.

The gradient of the objective function $J$ in (4.12) with respect to the parameters $\mathbf{W}^{(m)}$ and $\mathbf{b}^{(m)}$ at the top layer are computed as follows:

$$
\begin{aligned}
\frac{\partial J}{\partial \mathbf{W}^{(M)}} & =\frac{\partial J^{(M)}}{\partial \mathbf{W}^{(M)}}, \\
\frac{\partial J}{\partial \mathbf{b}^{(M)}} & =\frac{\partial J^{(M)}}{\partial \mathbf{b}^{(M)}},
\end{aligned}
$$

For other layers $m=1,2, \cdots, M-1$, they are computed as follows:

$$
\begin{aligned}
\frac{\partial J}{\partial \mathbf{W}^{(m)}} & =\frac{\partial J^{(M)}}{\partial \mathbf{W}^{(m)}}+\sum_{\ell=m}^{M-1} \omega^{(\ell)} h^{\prime}\left(J^{(\ell)}-\tau^{(\ell)}\right) \frac{\partial J^{(\ell)}}{\partial \mathbf{W}^{(m)}}, \\
\frac{\partial J}{\partial \mathbf{b}^{(m)}} & =\frac{\partial J^{(M)}}{\partial \mathbf{b}^{(m)}}+\sum_{\ell=m}^{M-1} \omega^{(\ell)} h^{\prime}\left(J^{(\ell)}-\tau^{(\ell)}\right) \frac{\partial J^{(\ell)}}{\partial \mathbf{b}^{(m)}}
\end{aligned}
$$


where $h^{\prime}(x)$ is the derivative of $h(x)$, and we set $h^{\prime}(x)=0$ for the non-differentiability point $x=0$.

For $1 \leq m \leq \ell \leq M$, we have

$$
\begin{aligned}
\frac{\partial J^{(\ell)}}{\partial \mathbf{W}^{(m)}} & =\frac{2}{N k_{1}} \sum_{i=1}^{N} \sum_{j=1}^{N} P_{i j}\left(\mathbf{L}_{i j}^{(m)} \mathbf{h}_{i}^{(m-1)^{T}}+\mathbf{L}_{j i}^{(m)} \mathbf{h}_{j}^{(m-1)^{T}}\right) \\
& -\frac{2 \alpha}{N k_{2}} \sum_{i=1}^{N} \sum_{j=1}^{N} Q_{i j}\left(\mathbf{L}_{i j}^{(m)} \mathbf{h}_{i}^{(m-1)^{T}}+\mathbf{L}_{j i}^{(m)} \mathbf{h}_{j}^{(m-1)^{T}}\right) \\
& +2 \beta\left(\frac{1}{N_{t}} \sum_{i=1}^{N_{t}} \mathbf{L}_{t i}^{(m)} \mathbf{h}_{t i}^{(m-1)^{T}}+\frac{1}{N_{s}} \sum_{i=1}^{N_{s}} \mathbf{L}_{s i}^{(m)} \mathbf{h}_{s i}^{(m-1)^{T}}\right) \\
& +2 \gamma \delta(\ell-m) \mathbf{W}^{(\ell)}, \\
\frac{\partial J^{(\ell)}}{\partial \mathbf{b}^{(m)}} & =\frac{2}{N k_{1}} \sum_{i=1}^{N} \sum_{j=1}^{N} P_{i j}\left(\mathbf{L}_{i j}^{(m)}+\mathbf{L}_{j i}^{(m)}\right)-\frac{2 \alpha}{N k_{2}} \sum_{i=1}^{N} \sum_{j=1}^{N} Q_{i j}\left(\mathbf{L}_{i j}^{(m)}+\mathbf{L}_{j i}^{(m)}\right) \\
& +2 \beta\left(\frac{1}{N_{t}} \sum_{i=1}^{N_{t}} \mathbf{L}_{t i}^{(m)}+\frac{1}{N_{s}} \sum_{i=1}^{N_{s}} \mathbf{L}_{s i}^{(m)}\right)+2 \gamma \delta(\ell-m) \mathbf{b}^{(\ell)},
\end{aligned}
$$

where the delta function $\delta(x)=0$ holds except $\delta(x)=1$ at point $x=0$, and the updating equations for all layers $1 \leq m \leq \ell-1$ are computed as follows:

$$
\begin{aligned}
& \mathbf{L}_{i j}^{(\ell)}=\left(\mathbf{h}_{i}^{(\ell)}-\mathbf{h}_{j}^{(\ell)}\right) \odot \varphi^{\prime}\left(\mathbf{z}_{i}^{(\ell)}\right), \\
& \mathbf{L}_{j i}^{(\ell)}=\left(\mathbf{h}_{j}^{(\ell)}-\mathbf{h}_{i}^{(\ell)}\right) \odot \varphi^{\prime}\left(\mathbf{z}_{j}^{(\ell)}\right), \\
& \mathbf{L}_{i j}^{(m)}=\left(\mathbf{W}^{(m+1)^{T}} \mathbf{L}_{i j}^{(m+1)}\right) \odot \varphi^{\prime}\left(\mathbf{z}_{i}^{(m)}\right), \\
& \mathbf{L}_{j i}^{(m)}=\left(\mathbf{W}^{(m+1)^{T}} \mathbf{L}_{j i}^{(m+1)}\right) \odot \varphi^{\prime}\left(\mathbf{z}_{j}^{(m)}\right), \\
& \mathbf{L}_{t i}^{(\ell)}=\left(\frac{1}{N_{t}} \sum_{j=1}^{N_{t}} \mathbf{h}_{t j}^{(\ell)}-\frac{1}{N_{s}} \sum_{j=1}^{N_{s}} \mathbf{h}_{s j}^{(\ell)}\right) \odot \varphi^{\prime}\left(\mathbf{z}_{t i}^{(\ell)}\right), \\
& \mathbf{L}_{s i}^{(\ell)}=\left(\frac{1}{N_{s}} \sum_{j=1}^{N_{s}} \mathbf{h}_{s j}^{(\ell)}-\frac{1}{N_{t}} \sum_{j=1}^{N_{t}} \mathbf{h}_{t j}^{(\ell)}\right) \odot \varphi^{\prime}\left(\mathbf{z}_{s i}^{(\ell)}\right), \\
& \mathbf{L}_{t i}^{(m)}=\left(\mathbf{W}^{(m+1)^{T}} \mathbf{L}_{t i}^{(m+1)}\right) \odot \varphi^{\prime}\left(\mathbf{z}_{t i}^{(m)}\right), \\
& \mathbf{L}_{s i}^{(m)}=\left(\mathbf{W}^{(m+1)^{T}} \mathbf{L}_{s i}^{(m+1)}\right) \odot \varphi^{\prime}\left(\mathbf{z}_{s i}^{(m)}\right) .
\end{aligned}
$$


We iteratively update $\left\{\mathbf{W}^{(m)}\right\}_{m=1}^{M}$ and $\left\{\mathbf{b}^{(m)}\right\}_{m=1}^{M}$ using the gradient descent method until the DSTML reaches a certain terminating condition.

\subsection{Deep Transfer Metric Learning with Autoencoder Reg- ularization}

Recent advances in the representation learning and deep learning have shown that the autoencoder and its variants have some good properties in preserving locality of data and recovering original data points from their resulting representations [122]. Inspired by this and in order to capture the local manifold structure of input data points as well as easily recover resulting data points in the metric space to their original forms, we adopt the autoencoder as a complementary regularizer to the DTML and DSTML methods and further introduce two new methods: deep transfer metric learning with autoencoder regularization (DTML-AE) and deeply supervised transfer metric learning with autoencoder regularization (DSTML-AE), respectively. Figure 4.3 shows the basic framework of the autoencoder used in our DTML-AE and DSTML-AE methods, which mainly consists of two parts, encoder and decoder.

\subsubsection{DTML with Autoencoder Regularization}

Given a data point $\mathbf{x} \in \mathbb{R}^{p^{(0)}}$ in the input space, the autoencoder first encodes it through the encoding part (i.e., first $M$ layers) to obtain the resulting representation $\mathbf{h}^{(M)}$ in the metric space, and then decodes $\mathbf{h}^{(M)}$ through the decoding part (i.e., last $M$ layers) to obtain the reconstruction $\widehat{\mathbf{x}}=\mathbf{h}^{(2 M)}$ of the $\mathbf{x}$ in the output space, i.e.,

$$
\begin{aligned}
& \mathbf{h}^{(M)}=f^{(M)}(\mathbf{x}) \in \mathbb{R}^{p^{(M)}}, \\
& \widehat{\mathbf{x}}=\mathbf{h}^{(2 M)}=f^{(2 M)}(\mathbf{x}) \in \mathbb{R}^{p^{(2 M)}},
\end{aligned}
$$




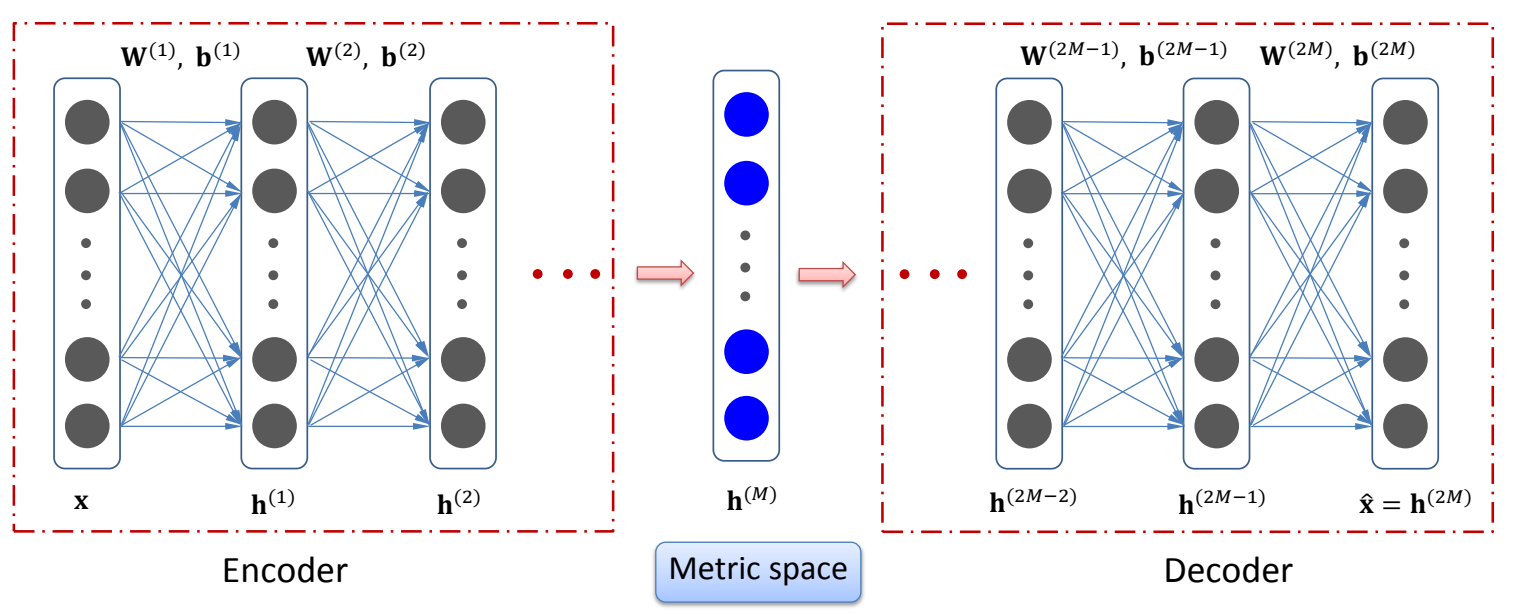

Figure 4.3: The network architecture of the autoencoder used in our methods. The $\mathrm{x}$ is a data point in the input space, $\mathbf{h}^{(M)}$ is the resulting representation of the $\mathbf{x}$ in the metric space, and the $\widehat{\mathbf{x}}=\mathbf{h}^{(2 M)}$ is the reconstruction of the point $\mathbf{x}$ in the output space.

in which the nonlinear mapping $f^{(2 M)}: \mathbb{R}^{p^{(0)}} \mapsto \mathbb{R}^{p^{(2 M)}}$ is a function parameterized by $\left\{\mathbf{W}^{(m)} \in \mathbb{R}^{p^{(m)} \times p^{(m-1)}}\right\}_{m=1}^{2 M}$ and $\left\{\mathbf{b}^{(m)} \in \mathbb{R}^{p^{(m)}}\right\}_{m=1}^{2 M}$, and we have constraints $p^{(m)}=$ $p^{(2 M-m)}, m=1,2, \cdots, 2 M$. These constraints are used to make sure that encoder and decoder networks are mirror symmetry for simplicity. In autoencoder term, we just minimize the reconstruction error between output layer $(m=2 M)$ and input layer $(m=0)$. By minimizing the reconstruction error $\left\|f^{(2 M)}(\mathbf{x})-\mathbf{x}\right\|_{2}^{2}$ of each data point of the training set, we can obtain the parameters of this autoencoder.

Then we exploit the autoencoder as a complementary regularizer to the DTML and formulate the proposed DTML-AE method as following optimization problem:

$$
\begin{aligned}
\min _{f^{(M)}, f^{(2 M)}} J & =S_{c}^{(M)}-\alpha S_{b}^{(M)}+\beta D_{t s}^{(M)}\left(\mathcal{X}_{t}, \mathcal{X}_{s}\right)+\gamma \sum_{m=1}^{2 M}\left(\left\|\mathbf{W}^{(m)}\right\|_{F}^{2}+\left\|\mathbf{b}^{(m)}\right\|_{2}^{2}\right) \\
& +\frac{\theta_{t}}{N_{t}} \sum_{i=1}^{N_{t}}\left\|f^{(2 M)}\left(\mathbf{x}_{t i}\right)-\mathbf{x}_{t i}\right\|_{2}^{2}+\frac{\theta_{s}}{N_{s}} \sum_{i=1}^{N_{s}}\left\|f^{(2 M)}\left(\mathbf{x}_{s i}\right)-\mathbf{x}_{s i}\right\|_{2}^{2} \\
& =J_{\mathrm{DTML}}+J_{\mathrm{AE}}
\end{aligned}
$$

where $J_{\mathrm{DTML}}$ is the objective function of the DTML in (4.7), and $J_{\mathrm{AE}}$ is the autoencoder 
regularization of the source domain and target domain data, i.e.,

$$
\begin{aligned}
J_{\mathrm{AE}} & =\frac{\theta_{t}}{N_{t}} \sum_{i=1}^{N_{t}}\left\|f^{(2 M)}\left(\mathbf{x}_{t i}\right)-\mathbf{x}_{t i}\right\|_{2}^{2}+\frac{\theta_{s}}{N_{s}} \sum_{i=1}^{N_{s}}\left\|f^{(2 M)}\left(\mathbf{x}_{s i}\right)-\mathbf{x}_{s i}\right\|_{2}^{2} \\
& +\gamma \sum_{m=M+1}^{2 M}\left(\left\|\mathbf{W}^{(m)}\right\|_{F}^{2}+\left\|\mathbf{b}^{(m)}\right\|_{2}^{2}\right)
\end{aligned}
$$

in which $\theta_{t}$ and $\theta_{s}$ are two positive regularization parameters to balance the importance of the autoencoder regularization to the whole objective function.

Next the gradient descent based method is applied to update the parameters $\left\{\mathbf{W}^{(m)}\right\}_{m=1}^{2 M}$ and $\left\{\mathbf{b}^{(m)}\right\}_{m=1}^{2 M}$. The partial derivative of the objective function $J$ in (4.22) with regard to $\mathbf{W}^{(m)}$ and $\mathbf{b}^{(m)}, m=1,2, \cdots, 2 M$, are presented as:

$$
\begin{gathered}
\frac{\partial J}{\partial \mathbf{W}^{(m)}}=u(M-m) \frac{\partial J_{\mathrm{DTML}}}{\partial \mathbf{W}^{(m)}}+\frac{\partial J_{\mathrm{AE}}}{\partial \mathbf{W}^{(m)}}, \\
\frac{\partial J}{\partial \mathbf{b}^{(m)}}=u(M-m) \frac{\partial J_{\mathrm{DTML}}}{\partial \mathbf{b}^{(m)}}+\frac{\partial J_{\mathrm{AE}}}{\partial \mathbf{b}^{(m)}},
\end{gathered}
$$

where $u(M-m)$ is the unit step function of the variable $m$, i.e., it holds that $u(M-m)=1$ for $1 \leq m \leq M$, and $u(M-m)=0$ for $M<m \leq 2 M$. The $\partial J_{\mathrm{DTML}} / \partial \mathbf{W}^{(m)}$ and $\partial J_{\mathrm{DTML}} / \partial \mathbf{b}^{(m)}$ can be calculated by (4.8) and (4.9), $m=1,2, \cdots, M$. Moreover, the $\partial J_{\mathrm{AE}} / \partial \mathbf{W}^{(m)}$ and $\partial J_{\mathrm{AE}} / \partial \mathbf{b}^{(m)}$ for $m=1,2, \cdots, 2 M$ are given as follows:

$$
\begin{aligned}
\frac{\partial J_{\mathrm{AE}}}{\partial \mathbf{W}^{(m)}} & =\frac{2 \theta_{t}}{N_{t}} \sum_{i=1}^{N_{t}} \mathbf{L}_{\mathrm{AE}, t i}^{(m)} \mathbf{h}_{t i}^{(m-1)^{T}}+\frac{2 \theta_{s}}{N_{s}} \sum_{i=1}^{N_{s}} \mathbf{L}_{\mathrm{AE}, s i}^{(m)} \mathbf{h}_{s i}^{(m-1)^{T}} \\
& +2 \gamma u(m-M-1) \mathbf{W}^{(m)} \\
\frac{\partial J_{\mathrm{AE}}}{\partial \mathbf{b}^{(m)}} & =\frac{2 \theta_{t}}{N_{t}} \sum_{i=1}^{N_{t}} \mathbf{L}_{\mathrm{AE}, t i}^{(m)}+\frac{2 \theta_{s}}{N_{s}} \sum_{i=1}^{N_{s}} \mathbf{L}_{\mathrm{AE}, s i}^{(m)} \\
& +2 \gamma u(m-M-1) \mathbf{b}^{(m)}
\end{aligned}
$$


in which the $\mathbf{L}_{\mathrm{AE}, t i}^{(m)}$ and $\mathbf{L}_{\mathrm{AE}, s i}^{(m)}$ are computed as

$$
\begin{aligned}
& \mathbf{L}_{\mathrm{AE}, t i}^{(2 M)}=\left(f^{(2 M)}\left(\mathbf{x}_{t i}\right)-\mathbf{x}_{t i}\right) \odot \varphi^{\prime}\left(\mathbf{z}_{t i}^{(2 M)}\right), \\
& \mathbf{L}_{\mathrm{AE}, s i}^{(2 M)}=\left(f^{(2 M)}\left(\mathbf{x}_{s i}\right)-\mathbf{x}_{s i}\right) \odot \varphi^{\prime}\left(\mathbf{z}_{s i}^{(2 M)}\right), \\
& \mathbf{L}_{\mathrm{AE}, t i}^{(m)}=\left(\mathbf{W}^{(m+1)^{T}} \mathbf{L}_{\mathrm{AE}, t i}^{(m+1)}\right) \odot \varphi^{\prime}\left(\mathbf{z}_{t i}^{(m)}\right), \\
& \mathbf{L}_{\mathrm{AE}, s i}^{(m)}=\left(\mathbf{W}^{(m+1)^{T}} \mathbf{L}_{\mathrm{AE}, s i}^{(m+1)}\right) \odot \varphi^{\prime}\left(\mathbf{z}_{s i}^{(m)}\right),
\end{aligned}
$$

for layer $m=1,2, \cdots, 2 M-1$; and $\mathbf{z}_{t i}^{(m)}$ is given as $\mathbf{z}_{t i}^{(m)}=\mathbf{W}^{(m)} \mathbf{h}_{t i}^{(m-1)}+\mathbf{b}^{(m)}$.

After having obtained the $\left\{\mathbf{W}^{(m)}\right\}_{m=1}^{2 M}$ and $\left\{\mathbf{b}^{(m)}\right\}_{m=1}^{2 M}$, the distance of a pair of data points $\mathbf{x}_{i}$ and $\mathbf{x}_{j}$ is measured in the deep metric space by

$$
d_{f^{(M)}}^{2}\left(\mathbf{x}_{i}, \mathbf{x}_{j}\right)=\left\|f^{(M)}\left(\mathbf{x}_{i}\right)-f^{(M)}\left(\mathbf{x}_{j}\right)\right\|_{2}^{2} .
$$

And this distance is used for verification and recognition.

\subsubsection{DSTML with Autoencoder Regularization}

Following the similar idea as in DTML-AE, the objective function of the DSTML-AE method can be formulated as:

$$
\min _{f^{(M)}, f^{(2 M)}} J=J_{\mathrm{DSTML}}+J_{\mathrm{AE}},
$$

where $J_{\mathrm{DSTML}}$ is the objective function of the DSTML in (4.12); and $J_{\mathrm{AE}}$ is the autoencoder regularization given by (4.23).

The gradient of the objective function $J$ of the DSTML-AE with respect to $\mathbf{W}^{(m)}$ and $\mathbf{b}^{(m)}$ are calculated by:

$$
\begin{gathered}
\frac{\partial J}{\partial \mathbf{W}^{(m)}}=u(M-m) \frac{\partial J_{\mathrm{DSTML}}}{\partial \mathbf{W}^{(m)}}+\frac{\partial J_{\mathrm{AE}}}{\partial \mathbf{W}^{(m)}}, \\
\frac{\partial J}{\partial \mathbf{b}^{(m)}}=u(M-m) \frac{\partial J_{\mathrm{DSTML}}}{\partial \mathbf{b}^{(m)}}+\frac{\partial J_{\mathrm{AE}}}{\partial \mathbf{b}^{(m)}}
\end{gathered}
$$

for the $m=1,2, \cdots, 2 M$, in which $\partial J_{\mathrm{DSTML}} / \partial \mathbf{W}^{(m)}, \partial J_{\mathrm{DSTML}} / \partial \mathbf{b}^{(m)}, \partial J_{\mathrm{AE}} / \partial \mathbf{W}^{(m)}$, and $\partial J_{\mathrm{AE}} / \partial \mathbf{b}^{(m)}$ are given by (4.14) - (4.17), (4.26), and (4.27). 


\subsection{Experiments}

In this section, we evaluate our DTML, DSTML, DTML-AE and DSTML-AE methods on three visual recognition tasks: face verification, person re-identification, and cross-domain handwritten digit recognition. We describe the detailed settings and experimental results below.

\subsubsection{Face Verification}

Face verification aims to decide whether a given pair of face images are from the same person or not. In this section, we conducted cross-dataset unconstrained face verification where face images were captured in wild conditions so that significant variations such as varying expression, pose, lighting, and background are included in the captured images.

\subsubsection{Datasets and Experimental Settings}

We used the Labeled Faces in the Wild (LFW) [69] and the Wide and Deep Reference (WDRef) [107] datasets for cross-dataset face verification. LFW is a challenging dataset which was developed for studying the problem of unconstrained face verification. There are 13233 face images of 5749 persons in the LFW dataset, which were collected from the internet and 1680 people have two or more images. This dataset was divided into 10 non-overlapping folds and the 10 -fold cross validation evaluation was used to test the performance of different face verification methods. For each fold, there are 300 matched (positive) pairs and 300 mismatched (negative) image pairs, respectively.

The WDRef [107] dataset contains 99773 face images of 2995 people, and 2065 subjects have at least 15 images. Since face image captured in WDRef are also from the internet, face images from these two datasets are generally similar. There is no overlapped subject between the LFW and WDRef datasets. In our experiments, we applied a subset 
which is randomly sampled from WDRef which consists of 15000 images (1500 subjects and each subject has 10 images) to learn the discriminative metric network.

In our settings, we selected the LFW dataset as the target domain data and the WDRef dataset as the source domain data. For each face image in these two datasets, we used the conventional local binary patterns (LBP) feature provided by the authors [107] to represent each face image. Specifically, we first extracted LBP descriptors at five facial landmarks with various scales, and then concatenated them to form a 5900-dimensional feature vector. To further remove the redundancy, each feature vector is reduced to 500 dimension by principal component analysis (PCA) where the projection is learnt on the source domain data. For our proposed DTML and DSTML methods, we designed a deep network with three layers $(M=2)$, and neural nodes from bottom to top layer are set as: $500 \rightarrow 400 \rightarrow$ 300. For the DTML-AE and DSTML-AE, we used an autoencoder of size $500 \rightarrow 400 \rightarrow$ $300 \rightarrow 400 \rightarrow 500$. The tanh function was used as the nonlinear activation function in these methods, and the parameters $\alpha, \beta, \gamma, \omega^{(1)}, \tau^{(1)}, k_{1}, k_{2}, \theta_{t}$ and $\theta_{s}$ were empirically set as $\alpha=0.1, \beta=10, \gamma=0.1, \omega^{(1)}=1, \tau^{(1)}=0, k_{1}=5, k_{2}=10, \theta_{t}=1$ and $\theta_{s}=1$, respectively. The initialized value of the learning rate $\lambda$ was set as 0.2 , and then it gradually reduced by multiplying a factor 0.95 in each iteration. We initialized $\mathbf{W}^{(m)}$ as a matrix with ones on the main diagonal and zeros elsewhere, and set the bias $\mathbf{b}^{(m)}$ as $\mathbf{0}$ for all $m=1,2, \cdots, M$.

\subsubsection{Results and Analysis}

We first compared our DTML with the shallow transfer metric learning (STML) method to show the advantage of the deep network. The STML is a special case of our DTML approach where the designed neural network only has two layers (i.e., input layer and output layer) and the linear activation function $\varphi(x)=x$ was used. Table 4.1 shows the 
Table 4.1: Mean verification rate with standard error (\%) of different transfer metric learning methods on the LFW dataset.

\begin{tabular}{|l|c|}
\hline Method & Accuracy (\%) \\
\hline \hline STML & $83.60 \pm 0.75$ \\
DTML & $85.58 \pm 0.61$ \\
DSTML & $87.32 \pm 0.67$ \\
DTML-AE & $87.02 \pm 0.47$ \\
DSTML-AE & $\mathbf{8 8 . 2 3} \pm \mathbf{0 . 4 5}$ \\
\hline \hline TML [64] & $83.25 \pm 0.53$ \\
DTDML [66] & $83.80 \pm 0.62$ \\
\hline
\end{tabular}

average verification rate with standard error of the DTML, DSTML, DTML-AE, DSTMLAE and STML. We can observe that DTML and its variants achieve better performance than STML. The reason is that DTML learns several hierarchical nonlinear transformations while STML learns only one linear transformation, so that the nonlinearity can be better exploited for face verification. Moreover, DSTML improves DTML by $1.7 \%$ in terms of the mean verification rate. This is because DSTML utilizes the label information from training sampled in the source domain at both the hidden layers and the top layer while DTML only exploit such information at the top layer, such that more discriminative information is exploited in DSTML. We also notice that the DTML-AE and DSTML-AE outperform the DTML and DSTML by $1.5 \%$ and $0.9 \%$, respectively. The reason is that DTML-AE and DSTML-AE can preserve the local manifold of data points in the learned metric space to help improve verification accuracy. Table 4.1 also lists the comparison of our methods and two existing transfer metric learning methods. The proposed deep transfer metric learning methods consistently perform better than these two transfer metric learning methods at a large gain.

To show the efficacy of knowledge transfer in our methods, we also compared STML, DTML, DSTML, DTML-AE and DSTML-AE with their corresponding metric learning 
Table 4.2: Mean verification rate with standard error (\%) of different metric learning methods with or without knowledge transfer on the LFW dataset.

\begin{tabular}{|l|c|c|}
\hline Method & Transfer & Accuracy (\%) \\
\hline \hline LMNN [4] & yes & $79.13 \pm 0.58$ \\
LMNN [4] & no & $77.27 \pm 0.52$ \\
\hline DDML [11] & yes & $84.63 \pm 0.71$ \\
DDML [11] & no & $83.16 \pm 0.80$ \\
\hline \hline STML & yes & $83.60 \pm 0.75$ \\
STML $(\beta=0)$ & no & $82.57 \pm 0.81$ \\
\hline DTML & yes & $85.58 \pm 0.61$ \\
DTML $(\beta=0)$ & no & $83.80 \pm 0.55$ \\
\hline DSTML & yes & $87.32 \pm 0.67$ \\
DSTML $(\beta=0)$ & no & $85.25 \pm 0.59$ \\
\hline DTML-AE & yes & $87.02 \pm 0.47$ \\
DTML-AE $(\beta=0)$ & no & $84.77 \pm 0.51$ \\
\hline DSTML-AE & yes & $\mathbf{8 8 . 2 3} \pm \mathbf{0 . 4 5}$ \\
DSTML-AE $(\beta=0)$ & no & $86.10 \pm 0.58$ \\
\hline
\end{tabular}

methods without knowledge transfer. To this end, we removed the MMD regularization term in STML, DTML, DSTML, DTML-AE and DSTML-AE, which means the value of the parameter $\beta$ is set as 0 in these methods. Table 4.2 shows the performance comparisons of these methods with and without knowledge transfer from source domain on the LFW dataset. We see that methods with knowledge transfer consistently improve those methods without knowledge transfer by $1 \% \sim 2.2 \%$ in terms of the mean verification accuracy even though the LFW and WDRef datasets are similar. Figure 4.4 also shows the ROC curves of several deep metric learning methods, and we can observe that transfer metric learning methods outperform those methods without knowledge transfer. 


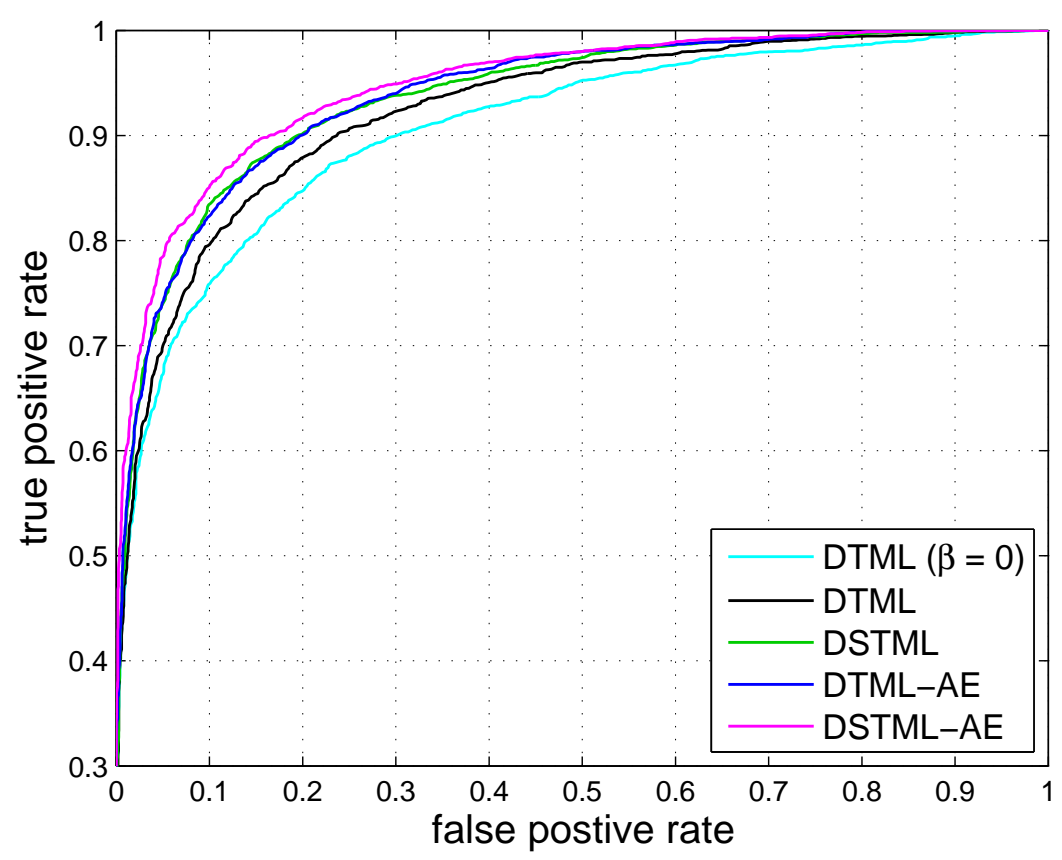

Figure 4.4: The ROC curves of several methods on LFW dataset.

\subsubsection{Person Re-Identification}

Person re-identification aims to recognize persons across multiple cameras without overlapping views. This task is very challenging because images of the same subject collected from multiple cameras are usually different due to variations of viewpoint, illumination, pose, resolution and occlusion. While many person re-identification methods have been proposed [92-94], there is no much work on cross-dataset person re-identification. In this subsection, we evaluate our deep transfer metric learning methods on cross-dataset person re-identification where only the label information of the source domain is used in the model learning.

\subsubsection{Datasets and Experimental Settings}

The VIPeR dataset [123] consists of 632 intra-personal image pairs captured outdoor by two different camera views, and most of them contain a viewpoint change of about 90 


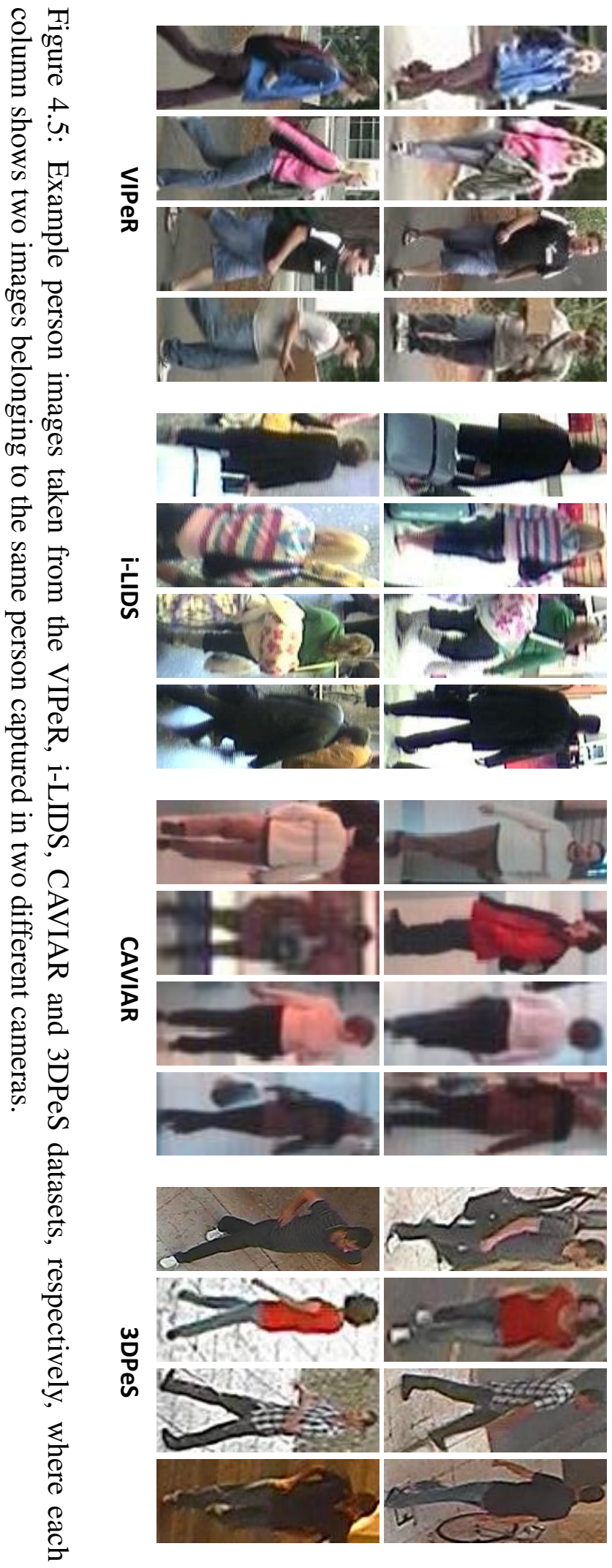


degrees. The i-LIDS dataset [124] contains 476 images of 119 people captured by five cameras at an airport, and each pedestrian has $2 \sim 8$ images. The CAVIAR dataset [125] contains 1220 images of 72 individuals from two different cameras in an indoor shopping mall, with $10 \sim 20$ images per person as well as large variations in resolutions. The 3DPeS dataset [126] has 1011 images of 192 persons collected from 8 different outdoor cameras with significant changes of viewpoint, and most of individuals appear in three different camera views. Figure 4.5 shows some example images from these four datasets, respectively.

In our experiments, all images from these datasets were scaled to $128 \times 48$ for feature extraction. For each image, we used two kinds of features descriptor: color and texture histograms. Following the settings in [94], each image was divided into six non-overlapping horizontal stripes. For each stripe, we extracted 16-bins histograms for each color channel of eight channels in the RGB (R, G, B), YUV (Y, U, V) and HSV (H, S) spaces, and computed uniform LBP with 8 neighbors and 16 neighbors respectively. Finally, color and texture histograms from these stripes were concatenated to form a 2580-dimensional feature vector for each image. Then PCA learnt on the source domain data was applied to reduce the dimension of target feature vector into a low dimensional subspace. We also adopted the single-Shot experiment setting $[92,94]$ to randomly split individuals in each dataset as training set and testing set, and repeated 10 times. For each partition, there were \#test subjects in the testing set, and one image for each person was randomly selected as the gallery set and the remaining images of this person were used as probe images. In DTML and DSTML, we also used a network with three layers $(M=2)$, and its neural nodes are given as: $200 \rightarrow 200 \rightarrow 100$ for all datasets. In DTML-AE and DSTML-AE, we employed an autoencoder of size $200 \rightarrow 200 \rightarrow 100 \rightarrow 200 \rightarrow 200$ for all datasets. The parameter $k_{1}$ and $k_{2}$ are set as 3 and 10, respectively. Note that if the number of image 
for a given person is less than 3 , all intra-class neighbors were used to compute the intraclass variations. For other parameters, we used the same settings as in face verification experiments on the LFW dataset.

\subsubsection{Results and Analysis}

Tables 4.3 - 4.6 show cross-dataset person re-identification performance of our methods on the VIPeR, i-LIDS, CAVIAR and 3DPeS datasets, respectively. The $L_{1}$ and $L_{2}$ are two baseline methods which directly use $L_{1}$ and $L_{2}$ norms to compute distance between a probe image and a gallery image in the target domain. DDML [11] is deep metric learning method which learns a distance metric on the source domain only. From these tables, we can observe that our DTML, DSTML, DTML-AE and DSTML-AE methods achieve better performance than the metric learning method without knowledge transfer in most of cases, which shows that transfer metric learning can help improve the performance of cross-dataset person re-identification. We also notice that DSTML-AE obtains the best performance for most cases, which indicates that exploiting discriminative information from more hidden layers rather than the single output layer is more effective to learn good distance metrics, and employing the autoencoder regularization term can capture the local manifold of data points in the learned metric space.

\subsubsection{Handwritten Digit Recognition}

Handwritten digit recognition is a classic example in machine learning and compute vision. We conducted several experiments on two well-known handwritten digit datasets for crossdomain handwritten digit recognition.

\subsubsection{Datasets and Experimental Settings}

The MNIST [5] handwritten digit dataset totally contains 70000 gray-scale images, in which the size of each image is $28 \times 28$ pixels. The USPS handwritten digit dataset consists 
Table 4.3: Top $r$ ranked matching accuracy (\%) on the VIPeR dataset with \#test $=316$ testing persons.

\begin{tabular}{|c|c|c|c|c|c|}
\hline Method & Source & $r=1$ & $r=5$ & $r=10$ & $r=30$ \\
\hline$L_{1}$ & - & 3.99 & 8.73 & 12.59 & 25.32 \\
\hline$L_{2}$ & - & 4.24 & 8.92 & 12.66 & 25.35 \\
\hline \multirow{3}{*}{ DDML [11] } & i-LIDS & 5.63 & 12.91 & 21.71 & 41.80 \\
\hline & CAVIAR & 5.91 & 13.53 & 19.86 & 37.92 \\
\hline & $3 \mathrm{DPeS}$ & 6.67 & 17.16 & 23.87 & 41.65 \\
\hline \multirow{3}{*}{$\begin{array}{l}\text { DTML } \\
(\beta=0)\end{array}$} & i-LIDS & 5.88 & 13.72 & 21.03 & 41.49 \\
\hline & CAVIAR & 6.02 & 13.81 & 20.33 & 38.46 \\
\hline & 3DPeS & 7.20 & 18.04 & 25.96 & 43.80 \\
\hline \multirow{3}{*}{ DTML } & i-LIDS & 6.68 & 15.73 & 23.20 & 46.42 \\
\hline & CAVIAR & 6.17 & 13.10 & 19.65 & 37.78 \\
\hline & $3 \mathrm{DPeS}$ & 8.51 & 19.40 & 27.59 & 47.91 \\
\hline \multirow{3}{*}{$\begin{array}{l}\text { DSTML } \\
(\beta=0)\end{array}$} & i-LIDS & 5.97 & 15.53 & 22.70 & 44.36 \\
\hline & CAVIAR & 6.36 & 16.30 & 23.52 & 40.44 \\
\hline & $3 \mathrm{DPeS}$ & 7.95 & 18.13 & 25.17 & 44.90 \\
\hline \multirow{3}{*}{ DSTML } & i-LIDS & 6.11 & 16.01 & 23.51 & 45.35 \\
\hline & CAVIAR & 6.61 & 16.93 & 24.40 & 41.55 \\
\hline & 3DPeS & 8.58 & 19.02 & 26.49 & 46.77 \\
\hline \multirow{3}{*}{$\begin{array}{l}\text { DTML-AE } \\
(\beta=0)\end{array}$} & i-LIDS & 6.52 & 15.06 & 21.97 & 45.23 \\
\hline & CAVIAR & 6.10 & 14.45 & 20.92 & 39.16 \\
\hline & 3DPeS & 8.39 & 19.61 & 27.57 & 46.85 \\
\hline \multirow{3}{*}{ DTML-AE } & i-LIDS & 6.86 & 16.15 & 24.06 & 47.93 \\
\hline & CAVIAR & 6.45 & 14.63 & 21.21 & 39.54 \\
\hline & 3DPeS & 8.87 & 20.03 & 28.93 & 49.30 \\
\hline \multirow{3}{*}{$\begin{array}{l}\text { DSTML-AE } \\
(\beta=0)\end{array}$} & i-LIDS & 6.20 & 16.11 & 23.38 & 45.26 \\
\hline & CAVIAR & 6.34 & 16.72 & 24.06 & 41.03 \\
\hline & 3DPeS & 8.22 & 18.16 & 25.95 & 46.60 \\
\hline \multirow{3}{*}{ DSTML-AE } & i-LIDS & 6.73 & 16.52 & 24.26 & 47.34 \\
\hline & CAVIAR & 6.80 & 17.15 & 26.13 & 43.36 \\
\hline & $3 \mathrm{DPeS}$ & 8.89 & 19.78 & 28.01 & 49.45 \\
\hline
\end{tabular}


Table 4.4: Top $r$ ranked matching accuracy (\%) on the i-LIDS dataset with \#test $=60$ testing persons.

\begin{tabular}{|c|c|c|c|c|c|}
\hline Method & Source & $r=1$ & $r=5$ & $r=10$ & $r=30$ \\
\hline$L_{1}$ & - & 16.51 & 28.41 & 38.28 & 69.32 \\
\hline$L_{2}$ & - & 16.30 & 28.25 & 38.40 & 69.77 \\
\hline \multirow{3}{*}{ DDML [11] } & VIPeR & 25.32 & 45.61 & 60.27 & 83.31 \\
\hline & CAVIAR & 25.67 & 45.03 & 61.38 & 82.56 \\
\hline & 3DPeS & 28.71 & 48.55 & 62.53 & 83.15 \\
\hline \multirow{3}{*}{$\begin{array}{l}\text { DTML } \\
(\beta=0)\end{array}$} & VIPeR & 26.27 & 47.59 & 62.62 & 85.07 \\
\hline & CAVIAR & 26.15 & 46.87 & 62.08 & 84.78 \\
\hline & $3 \mathrm{DPeS}$ & 30.23 & 51.60 & 65.21 & 85.53 \\
\hline \multirow{3}{*}{ DTML } & VIPeR & 28.90 & 51.43 & 65.47 & 87.23 \\
\hline & CAVIAR & 26.23 & 49.31 & 63.99 & 87.76 \\
\hline & 3DPeS & 31.01 & 54.51 & 65.96 & 88.66 \\
\hline \multirow{3}{*}{$\begin{array}{l}\text { DSTML } \\
(\beta=0)\end{array}$} & VIPeR & 27.50 & 49.13 & 59.92 & 82.89 \\
\hline & CAVIAR & 26.81 & 47.30 & 62.96 & 86.15 \\
\hline & 3DPeS & 31.44 & 53.23 & 66.15 & 87.29 \\
\hline \multirow{3}{*}{ DSTML } & VIPeR & 28.35 & 50.81 & 61.58 & 84.72 \\
\hline & CAVIAR & 28.37 & 58 & 59 & 88.68 \\
\hline & 3DPeS & 33.37 & 54.56 & 68.27 & 89.32 \\
\hline \multirow{3}{*}{$\begin{array}{l}\text { DTML-AE } \\
(\beta=0)\end{array}$} & VIPeR & 27.40 & 48.87 & 64.56 & 86.91 \\
\hline & CAVIAR & 27.15 & 48.00 & 63.61 & 86.53 \\
\hline & 3DPeS & 31.36 & 52.93 & 67.02 & 87.46 \\
\hline \multirow{3}{*}{ DTML-AE } & VIPeR & 29.43 & 52.26 & 66.01 & 87.85 \\
\hline & CAVIAR & 26.70 & 49.82 & 64.65 & 88.39 \\
\hline & 3DPeS & 31.25 & 55.10 & 66.59 & 89.11 \\
\hline \multirow{3}{*}{$\begin{array}{l}\text { DSTML-AE } \\
(\beta=0)\end{array}$} & VIPeR & 28.10 & 50.56 & 61.27 & 84.45 \\
\hline & CAVIAR & 28.06 & 49.43 & 64.15 & 88.23 \\
\hline & 3DPeS & 32.71 & 53.62 & 66.30 & 88.31 \\
\hline \multirow{3}{*}{ DSTML-AE } & VIPeR & 28.68 & 51.27 & 62.10 & 85.27 \\
\hline & CAVIAR & 28.89 & 50.10 & 65.13 & 89.30 \\
\hline & 3DPeS & 34.00 & 55.21 & 68.92 & 90.05 \\
\hline
\end{tabular}


Table 4.5: Top $r$ ranked matching accuracy (\%) on the CAVIAR dataset with \#test $=36$ testing persons.

\begin{tabular}{|c|c|c|c|c|c|}
\hline Method & Source & $r=1$ & $r=5$ & $r=10$ & $r=30$ \\
\hline$L_{1}$ & - & 20.65 & 36.44 & 48.52 & 88.34 \\
\hline$L_{2}$ & - & 20.19 & 36.43 & 48.55 & 87.69 \\
\hline \multirow{3}{*}{ DDML [11] } & VIPeR & 23.80 & 42.15 & 55.61 & 90.73 \\
\hline & i-LIDS & 22.72 & 41.36 & 56.92 & 90.06 \\
\hline & 3DPeS & 23.85 & 44.30 & 57.81 & 90.27 \\
\hline \multirow{3}{*}{$\begin{array}{l}\text { DTML } \\
(\beta=0)\end{array}$} & VIPeR & 23.71 & 42.57 & 56.15 & 90.55 \\
\hline & i-LIDS & 23.09 & 42.81 & 58.43 & 90.41 \\
\hline & 3DPeS & 25.11 & 46.71 & 59.69 & 91.99 \\
\hline \multirow{3}{*}{ DTML } & VIPeR & 23.88 & 42.36 & 55.60 & 92.12 \\
\hline & i-LIDS & 26.06 & 47.37 & 61.70 & 94.23 \\
\hline & $3 \mathrm{DPeS}$ & 26.10 & 47.80 & 61.31 & 93.02 \\
\hline \multirow{3}{*}{$\begin{array}{l}\text { DSTML } \\
(\beta=0)\end{array}$} & VIPeR & 23.93 & 42.85 & 56.76 & 91.32 \\
\hline & i-LIDS & 24.65 & 43.04 & 57.30 & 91.28 \\
\hline & 3DPeS & 26.70 & 47.51 & 62.19 & 92.34 \\
\hline \multirow{3}{*}{ DSTML } & VIPeR & 26.05 & 44.33 & 57.02 & 92.80 \\
\hline & i-LIDS & 25.91 & 44.47 & 58.88 & 93.33 \\
\hline & $3 \mathrm{DPeS}$ & 28.18 & 49.96 & 63.67 & 94.13 \\
\hline \multirow{3}{*}{$\begin{array}{l}\text { DTML-AE } \\
(\beta=0)\end{array}$} & VIPeR & 24.10 & 42.95 & 56.80 & 91.19 \\
\hline & i-LIDS & 23.44 & 43.20 & 58.92 & 91.05 \\
\hline & 3DPeS & 25.50 & 47.34 & 60.53 & 92.82 \\
\hline \multirow{3}{*}{ DTML-AE } & VIPeR & 24.73 & 43.27 & 57.68 & 93.03 \\
\hline & i-LIDS & 26.41 & 47.78 & 62.19 & 94.50 \\
\hline & 3DPeS & 26.55 & 48.01 & 61.66 & 93.41 \\
\hline \multirow{3}{*}{$\begin{array}{l}\text { DSTML-AE } \\
(\beta=0)\end{array}$} & VIPeR & 24.18 & 43.16 & 57.21 & 91.97 \\
\hline & i-LIDS & 25.10 & 43.57 & 57.83 & 92.19 \\
\hline & 3DPeS & 27.22 & 48.10 & 62.76 & 93.25 \\
\hline \multirow{3}{*}{ DSTML-AE } & VIPeR & 26.26 & 44.69 & 57.62 & 93.21 \\
\hline & i-LIDS & 26.35 & 45.23 & 59.50 & 93.54 \\
\hline & 3DPeS & 28.59 & 50.25 & 63.98 & 94.66 \\
\hline
\end{tabular}


Table 4.6: Top $r$ ranked matching accuracy $(\%)$ on the $3 \mathrm{DPeS}$ dataset with \#test $=95$ testing persons.

\begin{tabular}{|c|c|c|c|c|c|}
\hline Method & Source & $r=1$ & $r=5$ & $r=10$ & $r=30$ \\
\hline$L_{1}$ & - & 26.93 & 42.21 & 51.56 & 69.57 \\
\hline$L_{2}$ & - & 26.95 & 42.57 & 51.46 & 69.84 \\
\hline \multirow{3}{*}{ DDML [11] } & VIPeR & 29.56 & 51.03 & 61.71 & 78.62 \\
\hline & i-LIDS & 27.81 & 50.29 & 58.33 & 77.05 \\
\hline & CAVIAR & 30.32 & 49.36 & 58.92 & 79.61 \\
\hline \multirow{3}{*}{$\begin{array}{l}\text { DTML } \\
(\beta=0)\end{array}$} & VIPeR & 30.33 & 52.18 & 62.24 & 82.58 \\
\hline & i-LIDS & 29.12 & 50.07 & 59.99 & 78.59 \\
\hline & CAVIAR & 31.23 & 51.88 & 60.87 & 81.30 \\
\hline \multirow{3}{*}{ DTML } & VIPeR & 32.12 & 54.36 & 65.92 & 84.65 \\
\hline & i-LIDS & 32.11 & 52.08 & 61.63 & 79.45 \\
\hline & CAVIAR & 31.79 & 51.92 & 62.78 & 81.98 \\
\hline \multirow{3}{*}{$\begin{array}{l}\text { DSTML } \\
(\beta=0)\end{array}$} & VIPeR & 30.87 & 52.63 & 62.51 & 82.73 \\
\hline & i-LIDS & 31.03 & 51.85 & 62.34 & 83.11 \\
\hline & CAVIAR & 31.90 & 53.00 & 64.65 & 82.53 \\
\hline \multirow{3}{*}{ DSTML } & VIPeR & 32.51 & 52.97 & 12 & 83.08 \\
\hline & i-LIDS & 31.57 & 52.54 & 63.50 & 84.02 \\
\hline & CAVIAR & 32.53 & 54.29 & 65.28 & 84.72 \\
\hline \multirow{3}{*}{$\begin{array}{l}\text { DTML-AE } \\
(\beta=0)\end{array}$} & VIPeR & 30.57 & 52.51 & 62.59 & 83.10 \\
\hline & i-LIDS & 29.60 & 50.83 & 60.75 & 78.92 \\
\hline & CAVIAR & 31.58 & 52.25 & 61.31 & 82.06 \\
\hline \multirow{3}{*}{ DTML-AE } & VIPeR & 32.81 & 54.90 & 66.76 & 85.14 \\
\hline & i-LIDS & 32.46 & 52.82 & 62.37 & 80.31 \\
\hline & CAVIAR & 32.62 & 52.76 & 63.03 & 82.79 \\
\hline \multirow{3}{*}{$\begin{array}{l}\text { DSTML-AE } \\
(\beta=0)\end{array}$} & VIPeR & 31.05 & 52.88 & 62.95 & 83.27 \\
\hline & i-LIDS & 31.20 & 52.13 & 62.78 & 83.81 \\
\hline & CAVIAR & 32.18 & 53.44 & 65.00 & 83.56 \\
\hline \multirow{3}{*}{ DSTML-AE } & VIPeR & 32.82 & 53.39 & 63.51 & 83.70 \\
\hline & i-LIDS & 31.99 & 53.01 & 64.10 & 84.53 \\
\hline & CAVIAR & 33.05 & 54.75 & 66.20 & 85.69 \\
\hline
\end{tabular}




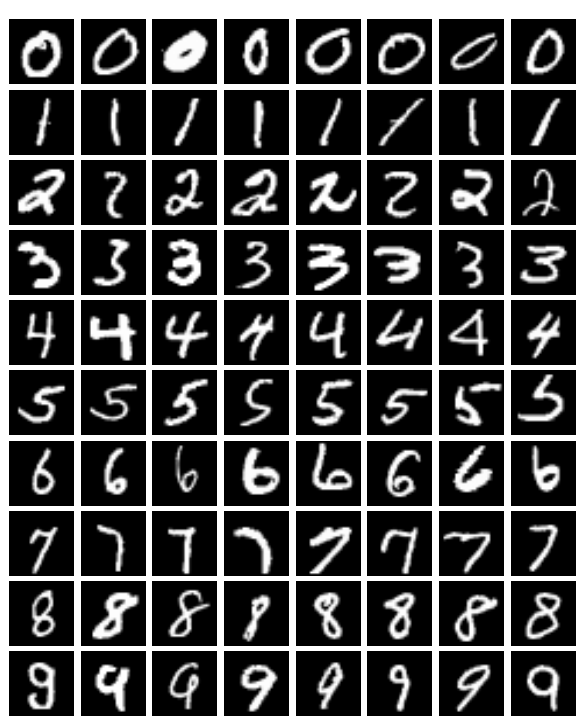

MNIST

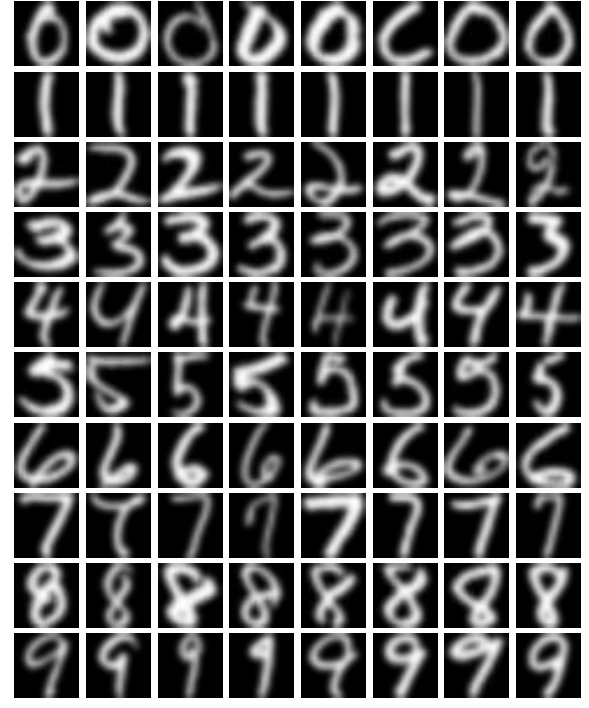

USPS

Figure 4.6: Handwritten digit images are from MNIST and USPS datasets.

of 9298 images of size $16 \times 16$ pixels. Both MNIST and USPS datasets enjoy ten semantic classes form digit 0 to digit 9 . Figure 4.6 shows several images randomly sampled from the MNIST and USPS datasets, where the images of these two datsets present the difference in appearance and distribution.

In the experiments, each image of the MNIST dataset was scaled to $16 \times 16$ pixels. And then each image of two datasets was represented by a 256-dimensional feature vector of raw pixels. Following the similar evaluation setting as in [127], we randomly chose 100 labeled images per class from the source domain (Note that these 1000 labeled images were also used as the gallery set in test.) and 1000 unlabeled images from the target domain to form the training set. In test, 10 images per category were sampled from the remaining samples of the target domain to form the probe set. We repeated this split 5 times and reported the average recognition accuracies for performance evaluation. Regarding the parameter settings, we adopted neural nodes of size $256 \rightarrow 200 \rightarrow 100$ for the DTML and DSTML methods, and employed the autoencoder of size $256 \rightarrow 200 \rightarrow 100 \rightarrow 200 \rightarrow 256$ for the 
Table 4.7: The average recognition accuracy (\%) of several methods on MNIST and USPS datasets. Note that the arrow " $\rightarrow$ " denotes the direction from source domain to target domain.

\begin{tabular}{|l|c|c|}
\hline Method & MNIST $\rightarrow$ USPS & USPS $\rightarrow$ MNIST \\
\hline \hline STML & $80.5 \pm 0.6$ & $72.8 \pm 0.9$ \\
STML $(\beta=0)$ & $71.3 \pm 0.7$ & $61.6 \pm 1.3$ \\
\hline DTML & $85.1 \pm 0.5$ & $76.2 \pm 0.8$ \\
DTML $(\beta=0)$ & $73.9 \pm 0.8$ & $65.3 \pm 1.1$ \\
\hline DSTML & $86.5 \pm 0.6$ & $78.5 \pm 0.7$ \\
DSTML $(\beta=0)$ & $74.4 \pm 0.9$ & $66.6 \pm 1.2$ \\
\hline DTML-AE & $87.2 \pm 0.7$ & $78.7 \pm 0.8$ \\
DTML-AE $(\beta=0)$ & $74.6 \pm 0.8$ & $67.3 \pm 1.0$ \\
\hline DSTML-AE & $\mathbf{8 8 . 7} \pm \mathbf{0 . 4}$ & $\mathbf{7 9 . 3} \pm \mathbf{0 . 6}$ \\
DSTML-AE $(\beta=0)$ & $75.8 \pm 0.5$ & $68.5 \pm 1.1$ \\
\hline \hline SGF [128] & $79.2 \pm 0.9$ & $71.1 \pm 0.7$ \\
GFK [129] & $82.6 \pm 0.8$ & $74.9 \pm 0.9$ \\
LSDT [127] & $79.3 \pm 0.8$ & $70.5 \pm 1.4$ \\
NLSDT [127] & $87.4 \pm 0.5$ & $79.1 \pm 0.8$ \\
\hline
\end{tabular}

DTML-AE and DSTML-AE methods. For other parameters, we followed the same settings as in face verification experiments.

\subsubsection{Results and Analysis}

The average recognition accuracy of our transfer metric learning methods, including STML, DTML, DSTML, DTML-AE and DSTML-AE on the two cross-domain settings (i.e., MNIST $\rightarrow$ USPS, and USPS $\rightarrow$ MNIST) are illustrated in Table 4.7. From these results, we can notice that all transfer metric learning methods consistently outperform their partners which only learn the distance metrics on the source domain. This further shows effectiveness of our proposed approaches on exploiting information of source domain to help cross-domain recognition. We also notice that 1) DSTML and DSTML-AE show better accuracies than DTML and DTML-AE; and 2) DTML-AE and DSTML-AE consistently 
outperform DTML and DSTML methods. The reason is that DSTML and DSTML-AE can make use of the label information on the hidden layers and the output layer to guide parameters learning, and DTML-AE and DSTML-AE can preserve the local manifold of data points in the learned metric space, respectively. In addition, Table 4.7 lists performance comparison of our methods and several state-of-the-art transfer learning methods, including SGF [128], GFK [129], LSDT [127] and NLSDT [127]. We can see that the DSTML-AE obtains the best accuracy, and our DTML-AE is comparable to NLSDT [127] on two cross-domain settings.

\subsubsection{Parameter Analysis}

In this subsection, we empirically investigate the parameter sensitivity of our methods on LFW and WDRef datasets. While there are several parameters, three of them $\left(\beta, \theta_{t}\right.$ and $\left.\theta_{s}\right)$ are the key parameters and others can be empirically determined by cross validation. Specifically, we fixed $\alpha, \gamma, k_{2}, \tau$ and $\omega$, and searched $\beta$ in $\{0,0.1,1,10,100\}$ and $\theta_{t}\left(\theta_{s}=\right.$ $\left.\theta_{t}\right)$ in $\{0,0.1,1,10,100\}$ by cross-validation.

\subsubsection{MMD Regularization $\beta$}

We first investigated the influence of the MMD regularization parameter $\beta$ to our transfer metric learning methods. We run DTML, DSTML, DTML-AE and DSTML-AE methods with varying values of $\beta$. Specifically, we individually picked $\beta$ from $\{0,0.1,1,10,100\}$ while fixing other parameters to their default settings. Figure 4.7 plots the mean verification accuracy of our four methods under different values of $\beta$. From this figure, we can observe that the larger values of $\beta$ gradually improve the performance of all methods. The reason is that the large $\beta$ can reduce the distribution difference of source domain and target domain. We also notice that our four methods achieve the relatively stable performance when $\beta$ 


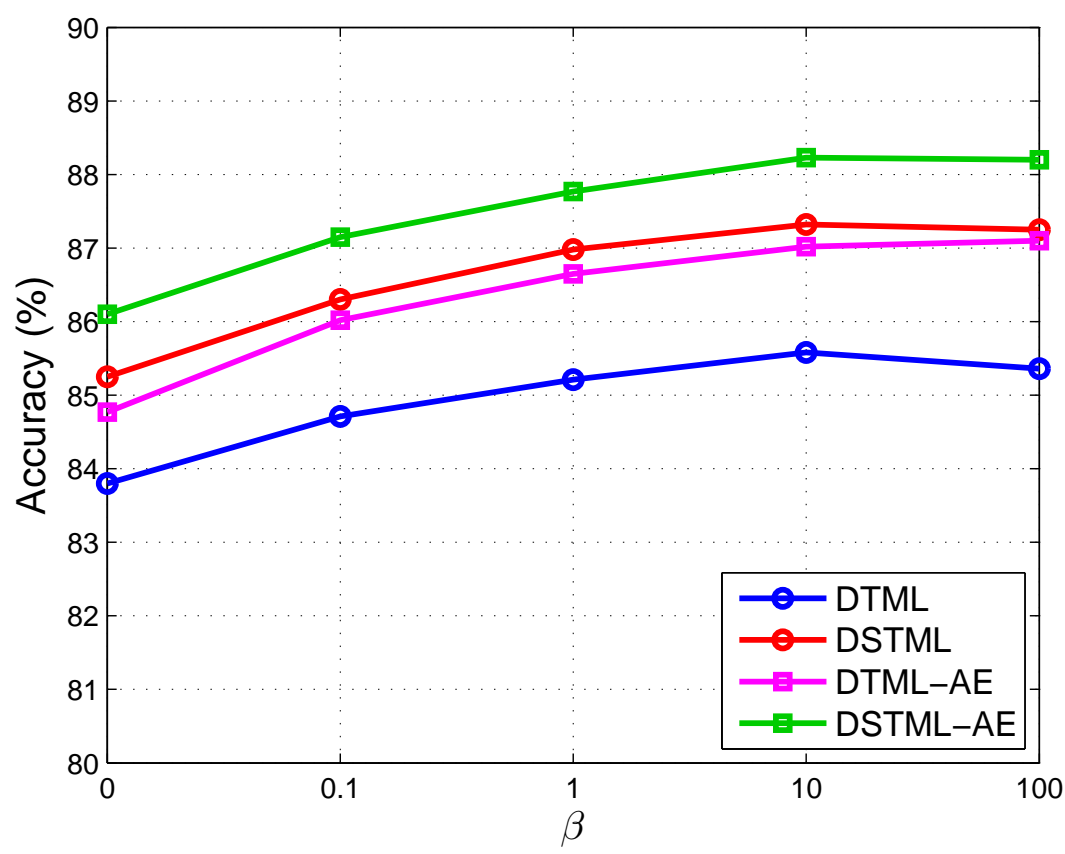

Figure 4.7: The mean accuracy of the DTML, DSTML, DTML-AE and DSTML-AE methods with varying values of $\beta$ on the LFW dataset.

falls into [1,100]. Thus, we can easily choose $\beta \in[1,100]$, and we set $\beta=10$ in our experiments to obtain stable results for other datasets.

\subsubsection{Autoencoder Regularization $\theta_{t}$ and $\theta_{s}$}

We then studied the sensitivity of the autoencoder regularization parameters $\theta_{t}$ and $\theta_{s}$ for the DTML-AE and DSTML-AE methods. We implemented the DTML-AE and DSTML$\mathrm{AE}$ approaches with various values of $\theta_{t}$ and $\theta_{s}$ in $\{0,0.1,1,10,100\}$ when other parameter$\mathrm{s}$ are fixed. In the experiments, we directly set $\theta_{t}=\theta_{s}$ to reduce the number of parameters in our methods. Figure 4.8 shows the mean accuracy of the DTML-AE and DSTML-AE methods with varying values of $\theta_{t}$ and $\theta_{s}$ on the LFW dataset. From this figure, we can see that our methods can achieve stable performance when $\theta_{t}$ and $\theta_{s}$ are selected from $[0.1,10]$, and $\theta_{t}, \theta_{s}=1$ reports the best mean accuracy. Thus, we simply set $\theta_{t}, \theta_{s}=1$ for other 


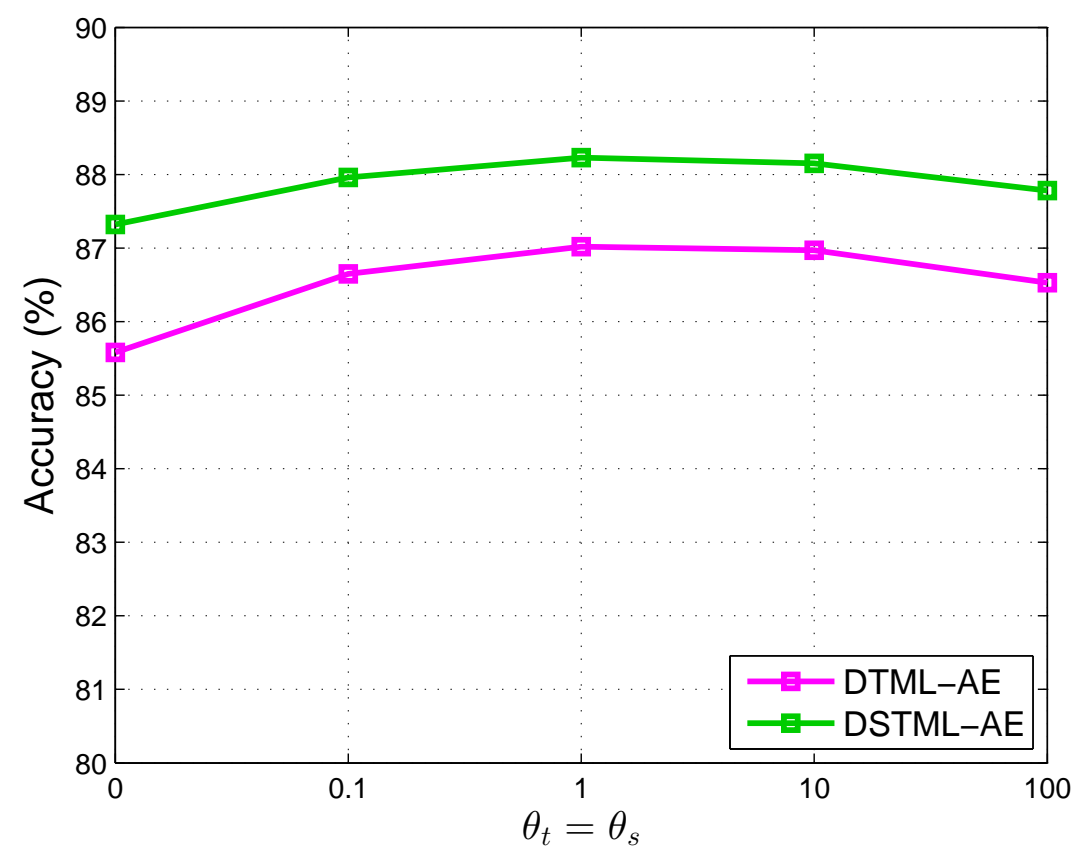

Figure 4.8: The mean accuracy of the DTML-AE and DSTML-AE methods with varying values of $\theta_{t}$ and $\theta_{s}\left(\theta_{t}=\theta_{s}\right)$ on the LFW dataset.

Table 4.8: The performance and time complexity of DTML versus different numbers of hidden layers on the LFW dataset.

\begin{tabular}{|l|c|c|c|c|}
\hline Layers & $M=1$ & $M=2$ & $M=3$ & $M=4$ \\
\hline \hline Accuracy (\%) & $83.60 \pm 0.75$ & $85.58 \pm 0.61$ & $85.72 \pm 0.59$ & $85.16 \pm 0.65$ \\
\hline Time (second) & 65.3 & 102.0 & 181.6 & 280.1 \\
\hline
\end{tabular}

datasets in our experiments. When $\theta_{t}$ and $\theta_{s}$ reach to zero, the performance reduces dramatically because the autoencoder regularization is not performed. When $\theta_{s}$ and $\theta_{s} \longrightarrow \infty$, the autoencoder regularization term of the DTML-AE and DSTML-AE methods is emphasized while labeled information and MMD regularization term are weakened such that their performance progressively decreases. 


\subsubsection{Layer Number $M$ and Computational Time}

We also investigated how the number of hidden layers influences the final performance and time complexity of DTML on the LFW dataset for face verification. We implemented our DTML using MATLAB codes on a PC whose hardware configuration comprises a 3.2GHz CPU (Intel Core i5-3470) and a 32GB RAM. We evaluated DTML with four different network structures: 1$)$ Two layers $(M=1)$ with neural nodes $500 \rightarrow 300 ; 2)$ Three layers $(M=2)$ with neural nodes $500 \rightarrow 400 \rightarrow 300 ; 3)$ Four layers $(M=3)$ with neural nodes $500 \rightarrow 400 \rightarrow 300 \rightarrow 300$; and 4) Five layers $(M=4)$ with neural nodes $500 \rightarrow 400 \rightarrow 300 \rightarrow 300 \rightarrow 300$ from bottom to top layer, respectively. Table 4.8 shows the performance and time complexity of DTML versus different numbers of hidden layers on the LFW dataset. We see that 1) DTML with $M=3$ reports the best performance and DTML with $M=2$ shows the second best performance in terms of the mean accuracy; and 2) the performance of DTML increases when $M$ varies from 1 to 3 and then decreases when $M$ varies from 3 to 4 . The reason is that overfitting occurs when $M$ varies from 3 to infinity due to having too many parameters relative to the limited amount of training data. We also observe that the training time of DTML increases when $M$ increases from 1 to 4 . Therefore we adopt $M=2$ for DTML on LFW dataset in our experiments considering the performance and time complexity.

\subsubsection{Discussion}

\subsubsection{Motivation of DSTML}

Since we aim to learn a deep distance metric to measure the similarity of samples, we expect that the similarity obtained in hidden layers is also useful. We make some constrains on the hidden layers to avoid useless outputs, so that we are able to directly influence the hidden layer update to favor highly discriminative distance metrics. Another motivation is 
to reduce the effect of vanishing and exploding gradient problem in training because each hidden layer has a direct supervision from ground truth.

\subsubsection{DTML versus CNN-based Method}

AlexNet [45] shows that the domain disparity can be well removed by using deep feature representation on objects. Generally, the performance of CNN-based method is better than that of our DTML when the CNN model is trained on the large-scale labeled dataset (e.g., ImageNet). While $\mathrm{CNN}$-based methods can learn robust feature representations, these methods require a large number of labeled data for training because there are extensive number of parameters to estimate. For some practical applications such as cross-modal face matching, it is not easy to collect such large number of labeled data for deep feature learning. In contrast, the number of parameters in our DTML is relatively small so that it can also work well for discriminative metric learning with small size of training samples. In addition, $\mathrm{CNN}$ focuses on learning generic feature representation for cross-domain recognition while our method aims to seek domain transfer similarity measure for cross-domain visual recognition. In this work, we present a deep similarity learning method to better measure the similarity of samples. Hence, our method is complementary to existing deep learning methods.

\subsubsection{Hand-crafted Feature versus Raw Data}

There are three reasons for us to choose Hand-crafted features such as LBP and histograms features: 1) These hand-crafted features have shown reasonably good performance in previous face verification and person re-identification applications; 2) We observe that handcrafted features with the learned deep metrics can obtain the favorable performance on several datasets; and 3) Existing deep learning methods usually require a large number of labeled training data to learn deep model such as $\mathrm{CNN}$ model. Our model can be considered 
as the fully connected part of the CNN model and our model doesn't utilize the convolutional and pooling layers. With the simplification, our method doesn't require many labeled training data because the number of parameters in our model is reduced.

\subsubsection{Batch Optimization versus Stochastic Algorithm}

There are two reasons that our methods employ a batch optimization method rather than stochastic gradient descent (SGD) algorithm: 1) our methods need to compute the intraclass compactness and the interclass separability of source domain; and 2) a single sample is usually noisy, while batch tend to average a little of the noise out. For the large-scale training data, we may try to employ minibatch SGD to made small enough to be computationally tractable.

\subsubsection{Motivation of DTML using $S_{c}$ and $S_{b}$}

We can employ any of the standard metric learning objectives. Our deep transfer metric learning framework is general and many alternative metric learning objectives can be used. In this work, we employ the graph embedding framework to define $S_{c}$ and $S_{b}$, where the marginal fisher analysis criterion was used to characterize the intra-class compactness and the interclass separability. There are two reasons for us to choose this criterion: 1) it doesn't require that the training samples obey the Gaussian distribution; and 2) it is a stronglysupervised learning approach so that discriminative information can be better exploited than weakly-supervised methods.

\subsubsection{Conflict Between Autoencoder and Transfer Learning}

The relation between the transformed data in the new space and the data in the original space is highlighted by adding the autoencoder term, and this autoencoder term has a negative effect on transfer between source and target if the autoencoder regularization parameter 
is set to a large value. From Figure 4.8, we can see that our methods can achieve stable performance when $\theta_{t}$ and $\theta_{s}$ are selected from $[0.1,10]$, and $\theta_{t}, \theta_{s}=1$ reports the best mean accuracy. Thus, we simply set $\theta_{t}, \theta_{s}=1$ for other datasets in our experiments. When $\theta_{t}$ and $\theta_{s}$ reach to zero, the performance reduces dramatically because the autoencoder regularization is not performed. When $\theta_{s}$ and $\theta_{s} \longrightarrow \infty$, the autoencoder regularization term of the DTML-AE and DSTML-AE methods is emphasized while labeled information and MMD regularization term are weakened such that their performance progressively decreases.

\subsection{Conclusion}

In this chapter, we have proposed a deep transfer metric learning (DTML) method for cross-dataset visual recognition. By learning a set of hierarchical nonlinear transformations which transfer discriminative knowledge from a labeled source domain to an unlabeled target domain, the proposed method can achieve better performance than existing linear metric learning methods. To better exploit the discriminative information from the source domain, we have further developed a deeply supervised transfer metric learning (DSTML) method by exploiting discriminative information from both the hidden layer and the top output layer to further improve the recognition performance. To preserve the local manifold of input data points in the metric space, we have introduced two new methods, DTML with autoencoder regularization (DTML-AE) and DSTML with autoencoder regularization (DSTML-AE). Experimental results are presented to show the effectiveness of the proposed methods. The key part of the work presented in this chapter was published in $[29,116]$.

In many real-world visual applications, it is possible to extract multiple feature representations for each image. Thus, it is desirable to learn distance metrics from these multiple feature representations such that more discriminative information can be exploited than those methods that learn a single distance metric from an individual feature. A 
widely used way is to concatenate different features into a new feature vector and then employ existing distance metric learning algorithms on this concatenated vector directly. However, this concatenation is not physically meaningful because each feature has its own statistical characteristic, and such a simple concatenation ignores the diversity of multiple features and cannot effectively explore the complementary information among the multiple features. To explore multiple feature representations in metric learning, the following chapter proposes a large-margin multi-metric learning $\left(\mathrm{LM}^{3} \mathrm{~L}\right)$ method and a local large-margin multi-metric learning $\left(\mathrm{L}^{2} \mathrm{M}^{3} \mathrm{~L}\right)$ method. 


\section{Chapter 5}

\section{Local Large-Margin Multi-Metric Learning}

In the previous two chapters, we have proposed several deep metric learning methods over single-view data. In this chapter, we present a large-margin multi-metric learning $\left(\mathrm{LM}^{3} \mathrm{~L}\right)$ method to jointly learn multiple global distance metrics under which the correlations of different feature representations of each sample are maximized. To better exploit the local structures of visual data, we further propose a local metric learning (LML) and a local largemargin multi-metric learning $\left(\mathrm{L}^{2} \mathrm{M}^{3} \mathrm{~L}\right)$ methods to learn a set of local metrics. Experimental results on face and kinship verification show the efficacy of the proposed methods.

\subsection{Introduction}

In many visual analysis applications, it is possible to extract multiple feature representations for each image and hence it is desirable to learn distance metrics from these multiple feature representations such that more discriminative information can be exploited than those learned from individual features. A widely used solution is to concatenate different features together into a new feature vector and then employ existing distance metric learning algorithms on this concatenated vector directly. However, this concatenation is not

The main work presented in this chapter has been published in [130,131]. 


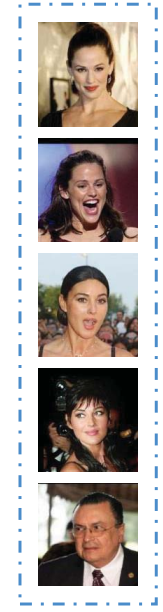

(a) Train Images (b) Features
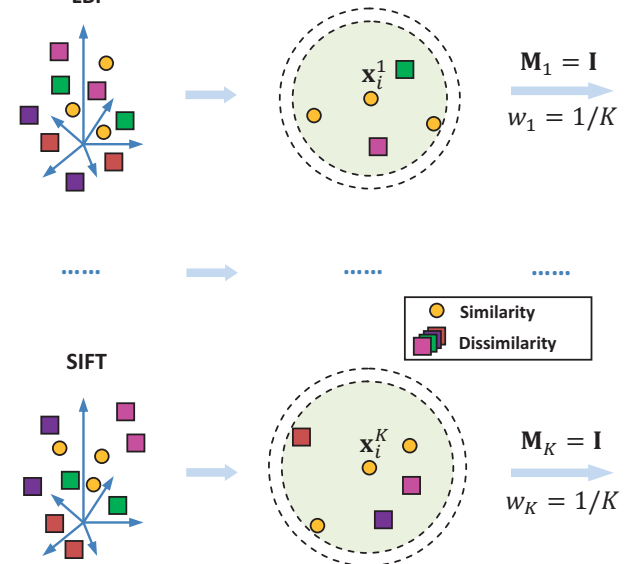

(c) Euclidean Metric

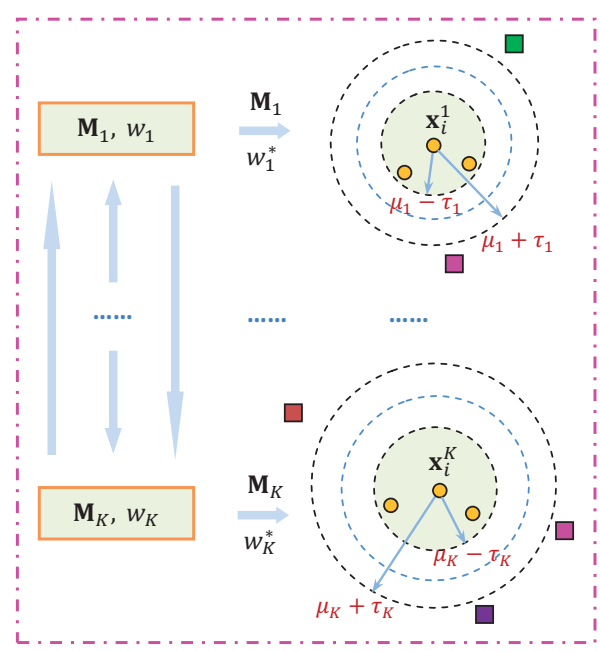

(d) $\mathrm{LM}^{3} \mathrm{~L}$

(e) Individual Metrics

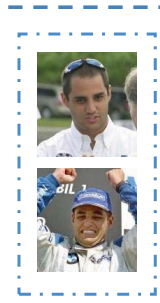

(f) Test Images

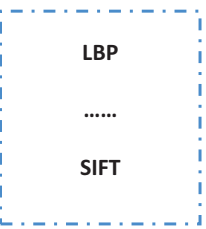

(g) Features

$$
d_{\mathbf{M}}^{2}\left(\mathbf{x}_{i}, \mathbf{x}_{j}\right)=\sum_{k=1}^{K} w_{k}^{*}\left(\mathbf{x}_{i}^{k}-\mathbf{x}_{j}^{k}\right)^{T} \mathbf{M}_{k}\left(\mathbf{x}_{i}^{k}-\mathbf{x}_{j}^{k}\right)
$$

(h) Verification

Figure 5.1: Illustration of our large-margin multi-metric learning $\left(\mathrm{LM}^{3} \mathrm{~L}\right)$ method for face verification, which jointly learns multiple distance metrics, one metric for each feature representations, and collaboratively optimizes the objective function over different features. (a) A training face image set; (b) The extracted $K$ different feature sets; (c) The distribution of these multiple feature representations in the Euclidean metric space; (d) The learning procedure of the $\mathrm{LM}^{3} \mathrm{~L}$; (e) The learned multiple distance metrics; (f) The test face image pairs; (g) The extracted multiple feature representations of the test face pairs; and (h) The resulting distance by fusing the multiple distance metrics learned by our method.

physically meaningful because each feature has its own statistical characteristic, and such a simple concatenation ignores the diversity of multiple features and cannot effectively explore the complementary information among the multiple features.

In this chapter, we first present a large-margin multi-metric learning $\left(\mathrm{LM}^{3} \mathrm{~L}\right)$ method for both face verification and kinship verification. Unlike the methods of learning a distance metric on the concatenated feature vectors, we collaboratively learn multiple distance metrics from multiple feature representations of data, where one distance metric is learned 
for each feature and the correlations of different feature representations of each sample are maximized, and under the learned metric spaces the distance of each positive pair is less than a small threshold and that of each negative pair is more than a large threshold, respectively. In addition, we also propose two local distance metric learning approaches, i.e., local metric learning (LML) and a local large-margin multi-metric learning $\left(\mathrm{L}^{2} \mathrm{M}^{3} \mathrm{~L}\right)$, to better exploit the local manifold structures of face images. Experimental results on three widely used face datasets show that our methods can obtain competitive results compared with state-of-the-art methods. Figure 5.1 illustrates the main pipeline of the proposed multi-metric learning methods.

\subsection{Large-Margin Multi-Metric Learning}

Notation Bold capital letters, e.g., $\mathbf{X}_{1}, \mathbf{X}_{2}$, represent matrices, and bold lower case letters, e.g., $\mathbf{x}_{1}, \mathbf{x}_{2}$, represent column vectors. Given a training set containing $N$ data points, i.e., $\mathcal{X}=\left\{\mathbf{x}_{i}\right\}_{i=1}^{N}$, each data point of this set can be easily represented by the multiple types of features, e.g., color, texture, shape, etc. Let $\mathcal{X}_{k}=\left\{\mathbf{x}_{i}^{k} \in \mathbb{R}^{d_{k}}\right\}_{i=1}^{N}$ be the $k$-th feature set of $\mathcal{X}$ from the $k$-th type of feature representation, and let $\mathbf{X}_{k}=\left[\mathbf{x}_{1}^{k}, \mathbf{x}_{2}^{k}, \cdots, \mathbf{x}_{N}^{k}\right]$ be the feature matrix of set $\mathcal{X}_{k}$, where $\mathbf{x}_{i}^{k}$ is the feature vector of the data point $\mathbf{x}_{i}$ in the $k$-th feature space, $k=1,2, \cdots, K ; K$ is the total number of types of features; and $d_{k}$ is feature dimension of $\mathbf{x}_{i}^{k}$.

\subsubsection{Problem Formulation}

For a feature set $\mathcal{X}_{k}=\left\{\mathbf{x}_{i}^{k} \in \mathbb{R}^{d_{k}}\right\}_{i=1}^{N}$ from the $k$-th feature representation, the squared Mahalanobis distance between a pair of samples $\mathbf{x}_{i}^{k}$ and $\mathbf{x}_{j}^{k}$ can be computed as:

$$
d_{\mathbf{M}_{k}}^{2}\left(\mathbf{x}_{i}^{k}, \mathbf{x}_{j}^{k}\right)=\left(\mathbf{x}_{i}^{k}-\mathbf{x}_{j}^{k}\right)^{T} \mathbf{M}_{k}\left(\mathbf{x}_{i}^{k}-\mathbf{x}_{j}^{k}\right),
$$


where $\mathbf{M}_{k} \in \mathbb{R}^{d_{k} \times d_{k}}$ is a positive definite matrix.

We seek a distance metric $\mathbf{M}_{k}$ such that the squared distance $d_{\mathbf{M}_{k}}^{2}\left(\mathbf{x}_{i}^{k}, \mathbf{x}_{j}^{k}\right)$ for a face pair $\mathbf{x}_{i}^{k}$ and $\mathbf{x}_{j}^{k}$ in the $k$ th feature space should be smaller than a given threshold $\mu_{k}-\tau_{k}$ $\left(\mu_{k}>\tau_{k}>0\right)$ if two samples are from the same subject, and larger than a threshold $\mu_{k}+\tau_{k}$ if these two samples are from different subjects, which can be formulated as the following constraints:

$$
y_{i j}\left(\mu_{k}-d_{\mathbf{M}_{k}}^{2}\left(\mathbf{x}_{i}^{k}, \mathbf{x}_{j}^{k}\right)\right)>\tau_{k},
$$

where pairwise label $y_{i j}=1$ if $\mathbf{x}_{i}^{k}$ and $\mathbf{x}_{j}^{k}$ are from the same category (or similar pair), and $y_{i j}=-1$ if they are from different categories (or dissimilar pair). The parameter $\mu_{k}$ is an absolute threshold to decide whether two samples are similar pair or not. The parameter $\tau_{k}$ is a positive slack variable to guarantee a margin (i.e., $2 \tau_{k}$ ) between a similar pair and a dissimilar pair.

To learn $\mathbf{M}_{k}$, we define the constraints in (5.2) using a hinge loss function, and formulate the following objective function to learn the $k$-th distance metric, named single metric learning (SML):

$$
\min _{\mathbf{M}_{k}} J_{k}=\sum_{i, j} h\left(\tau_{k}-y_{i j}\left(\mu_{k}-d_{\mathbf{M}_{k}}^{2}\left(\mathbf{x}_{i}^{k}, \mathbf{x}_{j}^{k}\right)\right)\right),
$$

where $h(x)=\max (x, 0)$ represents the hinge loss function. The objective function (5.3) penalizes the violation of the constraint $d_{\mathbf{M}_{k}}^{2}\left(\mathbf{x}_{i}^{k}, \mathbf{x}_{j}^{k}\right)>\mu_{k}-\tau_{k}$ for a similar pair and that of the constraint $d_{\mathbf{M}_{k}}^{2}\left(\mathbf{x}_{i}^{k}, \mathbf{x}_{j}^{k}\right)<\mu_{k}+\tau_{k}$ for a dissimilar pair by using the hinge loss.

Given a face image, it is easy to extract multiple features for each face image for multiple feature fusion. However, these features extracted from the same face image are usually highly correlated with one another even if they characterize face images from differen$\mathrm{t}$ aspects [132]. For multiple feature fusion, these highly correlated information should 
be preserved because they usually reflect the intrinsic information of samples. An important principle to perform multi-feature metric learning is to jointly learn multiple distance metrics by preserving the correlation between different feature pairs.

Previous studies [132-134] have shown that canonical correlation analysis (CCA) is an effective technique to fuse bi-modal features by maximizing their correlation, where a pair of projections are learned to map features in the original space into a latent space. There are two key advantages for CCA-based multiple feature fusion: 1) the effects of noise can be largely reduced and the signal-to-noise ratio (SNR) is enlarged [134], and 2) the most correlated information across multiple features is exploited and preserved [132]. Motivated by the success of CCA, we propose a large-margin multi-metric learning method to seek the commonality of multiple feature representations, which is consistent to the CCA-based multiple feature fusion methods [132-135].

The proposed large-margin multi-metric learning $\left(\mathrm{LM}^{3} \mathrm{~L}\right)$ method aims to learn $K$ distance metrics $\left\{\mathbf{M}_{k} \in \mathbb{R}^{d_{k} \times d_{k}}\right\}_{k=1}^{K}$ for a multi-feature dataset, such that

(i) The discriminative information from each single feature can be exploited as much as possible;

(ii) The differences of different feature representations of each sample in the learned distance metrics are minimized, because different features of each sample share the same semantic label.

Since the difference computation of the sample $\mathbf{x}_{i}$ from the $k$-th and $\ell$-th $(1 \leq k, \ell \leq K$, $k \neq \ell$ ) feature representations relies on the distance metrics $\mathbf{M}_{k}$ and $\mathbf{M}_{\ell}$, which could be different in dimensions, it is infeasible to compute them directly. To address this, we use an alternative constraint to reflect the relationships of different feature representations. Since the difference of $\mathbf{x}_{i}^{k}$ and $\mathbf{x}_{i}^{\ell}$, and that of $\mathbf{x}_{j}^{k}$ and $\mathbf{x}_{j}^{\ell}$ are expected to be minimized as much 
as possible, the distance between $\mathbf{x}_{i}^{k}$ and $\mathbf{x}_{j}^{k}$, and that of $\mathbf{x}_{i}^{\ell}$ and $\mathbf{x}_{j}^{\ell}$ are also expected to be as small as possible. Hence, we formulate the following objective function to constrain the interactions of different distance metrics in our $\mathrm{LM}^{3} \mathrm{~L}$ method:

$$
\begin{array}{rl}
\min _{\left\{\mathbf{M}_{k}, w_{k}\right\}_{k=1}^{K}} & J=\sum_{k=1}^{K} w_{k} J_{k}+\lambda \sum_{\substack{k, \ell=1 \\
k<\ell}}^{K} \sum_{i, j}\left(d_{\mathbf{M}_{k}}\left(\mathbf{x}_{i}^{k}, \mathbf{x}_{j}^{k}\right)-d_{\mathbf{M}_{\ell}}\left(\mathbf{x}_{i}^{\ell}, \mathbf{x}_{j}^{\ell}\right)\right)^{2}, \\
\text { s.t. } \quad & \sum_{k=1}^{K} w_{k}=1, w_{k} \geq 0, \lambda>0,
\end{array}
$$

where $w_{k}$ is a nonnegative weighting parameter to reflect the importance of the $k$-th feature in the whole objective function, and $\lambda$ weights the pairwise difference of the distance between two samples $\mathbf{x}_{i}$ and $\mathbf{x}_{j}$ in the learned distance metrics $\mathbf{M}_{k}$ and $\mathbf{M}_{\ell}$. The physical meaning of (5.4) is that we aim to learn $K$ distance metrics $\left\{\mathbf{M}_{k}\right\}_{k=1}^{K}$ under which the difference of feature representations of each pair of face samples is enforced to be as small as possible. The reason that a sample $x_{i}$ should be close in different feature spaces ( $k$ and $l$ ) is to seek a commonality of multiple features and make all the features more robust, which is consistent to the CCA-based multiple feature fusion methods.

In (5.4), the difference of distances $\left|d_{\mathbf{M}_{k}}\left(\mathbf{x}_{i}^{k}, \mathbf{x}_{j}^{k}\right)-d_{\mathbf{M}_{\ell}}\left(\mathbf{x}_{i}^{\ell}, \mathbf{x}_{j}^{\ell}\right)\right|$ doesn't imply that two samples $\mathbf{x}_{i}$ and $\mathbf{x}_{j}$ should be close in the same feature space. It only means that the distances of $\mathbf{x}_{i}$ and $\mathbf{x}_{j}$ should be close in the different feature spaces $(k \neq \ell)$. In addition, minimizing the metric difference $\left|d_{\mathbf{M}_{k}}\left(\mathbf{x}_{i}^{k}, \mathbf{x}_{j}^{k}\right)-d_{\mathbf{M}_{\ell}}\left(\mathbf{x}_{i}^{\ell}, \mathbf{x}_{j}^{\ell}\right)\right|$ doesn't mean that $d_{\mathbf{M}_{k}}\left(\mathbf{x}_{i}^{k}, \mathbf{x}_{j}^{k}\right)$ and $d_{\mathbf{M}_{\ell}}\left(\mathbf{x}_{i}^{\ell}, \mathbf{x}_{j}^{\ell}\right)$ will be minimized. By minimizing the $\left|d_{\mathbf{M}_{k}}\left(\mathbf{x}_{i}^{k}, \mathbf{x}_{j}^{k}\right)-d_{\mathbf{M}_{\ell}}\left(\mathbf{x}_{i}^{\ell}, \mathbf{x}_{j}^{\ell}\right)\right|$, both $d_{\mathbf{M}_{k}}\left(\mathbf{x}_{i}^{k}, \mathbf{x}_{j}^{k}\right)$ and $d_{\mathbf{M}_{\ell}}\left(\mathbf{x}_{i}^{\ell}, \mathbf{x}_{j}^{\ell}\right)$ will be reduced or increased simultaneously. Considering the constraint in (5.2), both $d_{\mathbf{M}_{k}}\left(\mathbf{x}_{i}^{k}, \mathbf{x}_{j}^{k}\right)$ and $d_{\mathbf{M}_{\ell}}\left(\mathbf{x}_{i}^{\ell}, \mathbf{x}_{j}^{\ell}\right)$ will be reduced for a similar pair, and will be increased for a dissimilar pair. As it is infeasible to compute the difference of $\mathbf{x}_{i}^{k}$ and $\mathbf{x}_{i}^{\ell}$ directly, we use the alternative constraint $\left|d_{\mathbf{M}_{k}}\left(\mathbf{x}_{i}^{k}, \mathbf{x}_{j}^{k}\right)-d_{\mathbf{M}_{\ell}}\left(\mathbf{x}_{i}^{\ell}, \mathbf{x}_{j}^{\ell}\right)\right|$ to make this problem solving more straightforward and obtain some feasible solution. Our experimental 
results show that minimizing $\left|d_{\mathbf{M}_{k}}\left(\mathbf{x}_{i}^{k}, \mathbf{x}_{j}^{k}\right)-d_{\mathbf{M}_{\ell}}\left(\mathbf{x}_{i}^{\ell}, \mathbf{x}_{j}^{\ell}\right)\right|$ indeed increases the correlation of $\mathbf{x}_{i}^{k}$ and $\mathbf{x}_{i}^{\ell}(k \neq \ell)$ in the learned metric spaces such that the commonality of multiple features is preserved for the robust face verification.

Having obtained the multiple distance metrics $\left\{\mathbf{M}_{k}\right\}_{k=1}^{K}$ and their weights $\left\{w_{k}\right\}_{k=1}^{K}$, the distance between two multi-feature data points $\mathbf{x}_{i}$ and $\mathbf{x}_{j}$ under the global metrics learned by the $\mathrm{LM}^{3} \mathrm{~L}$ can be computed as:

$$
\begin{aligned}
d_{\mathrm{LM}^{3} \mathrm{~L}}^{2}\left(\mathbf{x}_{i}, \mathbf{x}_{j}\right) & =\sum_{k=1}^{K} w_{k} d_{\mathbf{M}_{k}}^{2}\left(\mathbf{x}_{i}^{k}, \mathbf{x}_{j}^{k}\right) \\
& =\sum_{k=1}^{K} w_{k}\left(\mathbf{x}_{i}^{k}-\mathbf{x}_{j}^{k}\right)^{T} \mathbf{M}_{k}\left(\mathbf{x}_{i}^{k}-\mathbf{x}_{j}^{k}\right) .
\end{aligned}
$$

The trivial solution of (5.4) is $w_{k}=1$, which corresponds to the minimum $J_{k}$ over different feature representations, and $w_{k}=0$ otherwise. This solution means that only one single feature that yields the best verification accuracy is selected, which does not satisfy our objective on exploring the complementary property of multi-feature data.

To address this shortcoming, we modify $w_{k}$ to be $w_{k}^{p}$, where $p>1$ and $w_{k}^{p}$ is the $p$-th power of $w_{k}$, and the new objective function can be rewritten as:

$$
\begin{array}{rl}
\min _{\left\{\mathbf{M}_{k}, w_{k}\right\}_{k=1}^{K}} & J=\sum_{k=1}^{K} w_{k}^{p} J_{k}+\lambda \sum_{\substack{k, \ell=1 \\
k<\ell}}^{K} \sum_{i, j}\left(d_{\mathbf{M}_{k}}\left(\mathbf{x}_{i}^{k}, \mathbf{x}_{j}^{k}\right)-d_{\mathbf{M}_{\ell}}\left(\mathbf{x}_{i}^{\ell}, \mathbf{x}_{j}^{\ell}\right)\right)^{2}, \\
\text { s.t. } \quad & \sum_{k=1}^{K} w_{k}=1, w_{k} \geq 0, \lambda>0 .
\end{array}
$$

When $p=1$, it is not easy to obtain the optimal $w_{k}$. From (5.17), we obtain $J_{1}=J_{2}=$ $\cdots=J_{K}=\eta$, then $w_{k}$ can be an arbitrary value in the interval $[0,1]$. Thus, the trivial solution is $w_{k}=1$ for the minimum $J_{k}$ over different feature representations, and $w_{k}=0$ otherwise. When $p>1$, we obtain a closed-form solution of $w_{k}$ by (5.19), and each feature representation has a particular contribution to the final metric learning. 


\subsubsection{Alternating Optimization}

It is non-trivial to seek a global optimal solution to (5.6) because there are $K$ metrics to be learned simultaneously. In this work, we employ an iterative method by using the alternating optimization method to obtain a local optimal solution. The alternating optimization learns $\mathbf{M}_{k}$ and $w_{k}$ in an iterative manner. In our experiments, we randomly select the order of different features to start the optimization procedure and our tests show that the influence of this order is not critical to the final verification performance.

Step 1: Fix $\mathbf{w}=\left[w_{1}, w_{2}, \cdots, w_{K}\right]$, Update $\mathbf{M}_{k}$ With the fixed $\mathbf{w}$, we can cyclically optimize (5.6) over different features. We sequentially optimize $\mathbf{M}_{k}$ with the fixed $\mathbf{M}_{1}$, $\cdots, \mathbf{M}_{k-1}, \mathbf{M}_{k+1}, \cdots, \mathbf{M}_{K}$. Hence, (5.6) can be rewritten as:

$$
\min _{\mathbf{M}_{k}} J=A_{k}+w_{k}^{p} J_{k}+\lambda \sum_{\substack{\ell=1 \\ \ell \neq k}}^{K} \sum_{i, j}\left(d_{\mathbf{M}_{k}}\left(\mathbf{x}_{i}^{k}, \mathbf{x}_{j}^{k}\right)-d_{\mathbf{M}_{\ell}}\left(\mathbf{x}_{i}^{\ell}, \mathbf{x}_{j}^{\ell}\right)\right)^{2},
$$

where $A_{k}$ is a constant term.

To learn the distance metric $\mathbf{M}_{k}$, we employ a gradient-based scheme. After some algebraic simplification, we can obtain the gradient as:

$$
\frac{\partial J}{\partial \mathbf{M}_{k}}=w_{k}^{p} \sum_{i, j} y_{i j} h^{\prime}\left(z_{i j}\right) \mathbf{C}_{i j}^{k}+\lambda \sum_{\substack{\ell=1 \\ \ell \neq k}}^{K} \sum_{i, j}\left(1-\frac{d_{\mathbf{M}_{\ell}}\left(\mathbf{x}_{i}^{\ell}, \mathbf{x}_{j}^{\ell}\right)}{d_{\mathbf{M}_{k}}\left(\mathbf{x}_{i}^{k}, \mathbf{x}_{j}^{k}\right)}\right) \mathbf{C}_{i j}^{k},
$$

where $z_{i j}$ and $\mathbf{C}_{i j}^{k}$ can be calculated respectively by:

$$
\begin{aligned}
& \mathbf{C}_{i j}^{k}=\left(\mathbf{x}_{i}^{k}-\mathbf{x}_{j}^{k}\right)\left(\mathbf{x}_{i}^{k}-\mathbf{x}_{j}^{k}\right)^{T}, \\
& z_{i j}=\tau_{k}-y_{i j}\left(\mu_{k}-d_{\mathbf{M}_{k}}^{2}\left(\mathbf{x}_{i}^{k}, \mathbf{x}_{j}^{k}\right)\right) .
\end{aligned}
$$

The $\mathbf{C}_{i j}^{k}$ denotes the outer product of pairwise differences. $h^{\prime}(x)$ is the derivative of $h(x)$, and we handle the non-differentiability of $h(x)$ at $x=0$ by adopting a smooth hinge 
function as in $[115,136]$. In addition, we use some derivations given as:

$$
\begin{aligned}
\frac{\partial}{\partial \mathbf{M}_{k}} d_{\mathbf{M}_{k}}\left(\mathbf{x}_{i}^{k}, \mathbf{x}_{j}^{k}\right) & =\frac{1}{2 d_{\mathbf{M}_{k}}\left(\mathbf{x}_{i}^{k}, \mathbf{x}_{j}^{k}\right)} \mathbf{C}_{i j}^{k} \\
\frac{\partial}{\partial \mathbf{M}_{k}}\left(d_{\mathbf{M}_{k}}\left(\mathbf{x}_{i}^{k}, \mathbf{x}_{j}^{k}\right)-d_{\mathbf{M}_{\ell}}\left(\mathbf{x}_{i}^{\ell}, \mathbf{x}_{j}^{\ell}\right)\right)^{2} & =2\left(d_{\mathbf{M}_{k}}\left(\mathbf{x}_{i}^{k}, \mathbf{x}_{j}^{k}\right)-d_{\mathbf{M}_{\ell}}\left(\mathbf{x}_{i}^{\ell}, \mathbf{x}_{j}^{\ell}\right)\right) \frac{\partial}{\partial \mathbf{M}_{k}} d_{\mathbf{M}_{k}}\left(\mathbf{x}_{i}^{k}, \mathbf{x}_{j}^{k}\right) \\
& =\left(1-\frac{d_{\mathbf{M}_{\ell}}\left(\mathbf{x}_{i}^{\ell}, \mathbf{x}_{j}^{\ell}\right)}{d_{\mathbf{M}_{k}}\left(\mathbf{x}_{i}^{k}, \mathbf{x}_{j}^{k}\right)}\right) \mathbf{C}_{i j}^{k}
\end{aligned}
$$

Then, matrix $\mathbf{M}_{k}$ can be obtained by using a gradient descent algorithm:

$$
\mathbf{M}_{k}=\mathbf{M}_{k}-\beta \frac{\partial J}{\partial \mathbf{M}_{k}}
$$

where $\beta$ is the learning rate.

In practice, directly optimizing the Mahalanobis distance metric $\mathbf{M}_{k}$ may suffer from slow convergence and overfitting problems if the data points are very high of dimension and the number of training samples is insufficient. Therefore, we propose an alternative method to jointly perform dimensionality reduction and metric learning, which means a low-rank linear projection matrix $\mathbf{L}_{k} \in \mathbb{R}^{s_{k} \times d_{k}}\left(s_{k}<d_{k}\right)$ is learned to project each sample $\mathbf{x}_{i}^{k}$ from the high-dimensional input space to a low-dimensional embedding space, where the distance metric $\mathbf{M}_{k}=\mathbf{L}_{k}{ }^{T} \mathbf{L}_{k}$. Then, we differentiate the objective function $J$ with respect to $\mathbf{L}_{k}$, and obtain the gradient as follows:

$$
\frac{\partial J}{\partial \mathbf{L}_{k}}=2 \mathbf{L}_{k}\left[w_{k}^{p} \sum_{i, j} y_{i j} h^{\prime}\left(z_{i j}\right) \mathbf{C}_{i j}^{k}+\lambda \sum_{\substack{\ell=1 \\ \ell \neq k}}^{K} \sum_{i, j}\left(1-\frac{d_{\mathbf{M}_{\ell}}\left(\mathbf{x}_{i}^{\ell}, \mathbf{x}_{j}^{\ell}\right)}{d_{\mathbf{M}_{k}}\left(\mathbf{x}_{i}^{k}, \mathbf{x}_{j}^{k}\right)}\right) \mathbf{C}_{i j}^{k}\right] .
$$

Lastly, the matrix $\mathbf{L}_{k}$ can be obtained by using a gradient descent rule:

$$
\mathbf{L}_{k}=\mathbf{L}_{k}-\beta \frac{\partial J}{\partial \mathbf{L}_{k}}
$$

To make sure the learned metric $\mathbf{M}_{k}$ is a positive semidefinite matrix after each iteration, we clip the spectrum of $\mathbf{M}_{k}=\mathbf{L}_{k}^{T} \mathbf{L}_{k}$ by singular value decomposition. 
Step 2: Fix $\left\{\mathbf{M}_{k}\right\}_{k=1}^{K}$, Update $\mathbf{w}=\left[w_{1}, w_{2}, \cdots, w_{K}\right]$ Then, we update $\mathbf{w}$ with the fixed $\left\{\mathbf{M}_{k}\right\}_{k=1}^{K}$ using the method of Lagrange multipliers. We construct a Lagrange function as follows:

$$
L(\mathbf{w}, \eta)=\sum_{k=1}^{K} w_{k}^{p} J_{k}+\lambda \sum_{\substack{k, \ell=1 \\ k<\ell}}^{K} \sum_{i, j}\left(d_{\mathbf{M}_{k}}\left(\mathbf{x}_{i}^{k}, \mathbf{x}_{j}^{k}\right)-d_{\mathbf{M}_{\ell}}\left(\mathbf{x}_{i}^{\ell}, \mathbf{x}_{j}^{\ell}\right)\right)^{2}-\eta\left(\sum_{k=1}^{K} w_{k}-1\right) .
$$

Let $\frac{\partial L(\mathbf{w}, \eta)}{\partial w_{k}}=0$ and $\frac{\partial L(\mathbf{w}, \eta)}{\partial \eta}=0$, we have

$$
\begin{aligned}
& \frac{\partial L(\mathbf{w}, \eta)}{\partial w_{k}}=p w_{k}^{p-1} J_{k}-\eta=0 \\
& \frac{\partial L(\mathbf{w}, \eta)}{\partial \eta}=\sum_{k=1}^{K} w_{k}-1=0 .
\end{aligned}
$$

According to (5.17) and (5.18), $w_{k}$ can be updated as follows:

$$
w_{k}=\frac{\left(1 / J_{k}\right)^{1 /(p-1)}}{\sum_{k=1}^{K}\left(1 / J_{k}\right)^{1 /(p-1)}} .
$$

We repeat the above two steps until the algorithm meets a certain convergence condition. The proposed $\mathrm{LM}^{3} \mathrm{~L}$ algorithm is summarized in Algorithm 3, where $\mathbf{I} \in \mathbb{R}^{s_{k} \times d_{k}}$ is a matrix with 1's on the diagonal and zeros elsewhere.

\subsection{Local Large-Margin Multi-Metric Learning}

\subsubsection{Local Metric Learning}

Considering a single feature set $\mathcal{X}_{k}=\left\{\mathbf{x}_{i}^{k} \in \mathbb{R}^{d_{k}}\right\}_{i=1}^{N}$ of $\mathcal{X}$ which is represented by the $k$-th type of feature, the squared Mahalanobis distance between a pair of samples $\mathbf{x}_{i}^{k}$ and $\mathbf{x}_{j}^{k}$ under a specific local metric $\mathbf{M}_{k}^{(q)} \in \mathbb{R}^{d_{k} \times d_{k}}, q=1,2, \cdots, Q_{k}$, can be calculated by:

$$
d_{\mathbf{M}_{k}^{(q)}}^{2}\left(\mathbf{x}_{i}^{k}, \mathbf{x}_{j}^{k}\right)=\left(\mathbf{x}_{i}^{k}-\mathbf{x}_{j}^{k}\right)^{T} \mathbf{M}_{k}^{(q)}\left(\mathbf{x}_{i}^{k}-\mathbf{x}_{j}^{k}\right)
$$




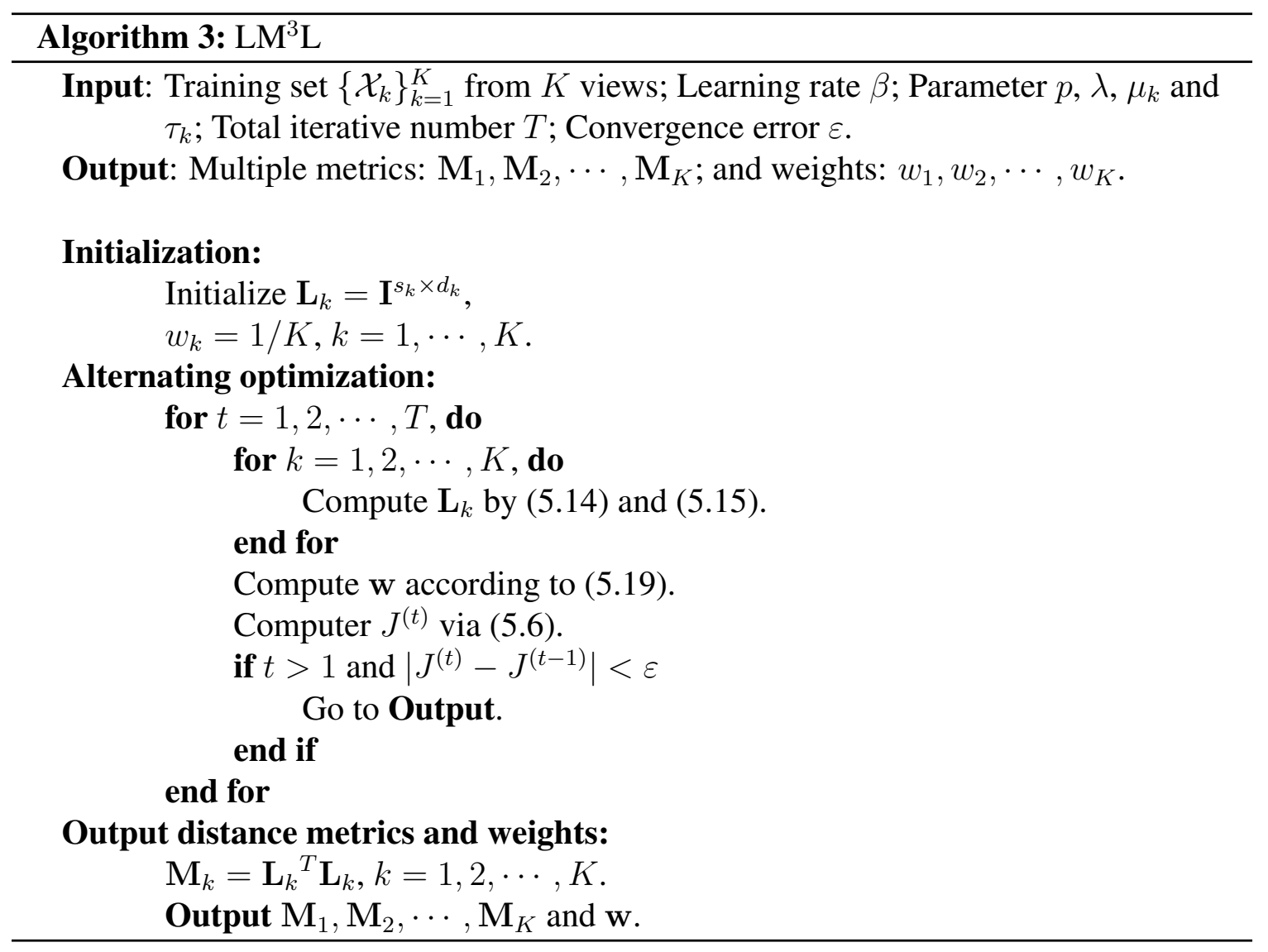

where $\mathbf{M}_{k}^{(q)} \in \mathbb{R}^{d_{k} \times d_{k}}$ is a positive semi-definite (PSD) matrix $\left(\mathbf{M}_{k}^{(q)} \succeq \mathbf{0}\right)$, and $Q_{k}$ is the total number of local distance metrics corresponding to the $k$-th type of feature representation.

Based on a set of local distance metrics $\left\{\mathbf{M}_{k}^{(q)}\right\}_{q=1}^{Q_{k}}$, the distance of each sample pair $\mathbf{x}_{i}^{k}$ and $\mathbf{x}_{j}^{k}$ can be defined as a convex combination by soft dividing the whole input space into different regions:

$$
\begin{aligned}
d_{\left\{\mathbf{M}_{k}^{(q)}\right\}_{q=1}^{Q_{k}}}^{2}\left(\mathbf{x}_{i}^{k}, \mathbf{x}_{j}^{k}\right) & =\sum_{q=1}^{Q_{k}} \alpha_{k}^{(q)}\left(\mathbf{x}_{i}^{k}, \mathbf{x}_{j}^{k}\right) d_{\mathbf{M}_{k}^{(q)}}^{2}\left(\mathbf{x}_{i}^{k}, \mathbf{x}_{j}^{k}\right) \\
& =\left(\mathbf{x}_{i}^{k}-\mathbf{x}_{j}^{k}\right)^{T}\left(\sum_{q=1}^{Q_{k}} \alpha_{k}^{(q)}\left(\mathbf{x}_{i}^{k}, \mathbf{x}_{j}^{k}\right) \mathbf{M}_{k}^{(q)}\right)\left(\mathbf{x}_{i}^{k}-\mathbf{x}_{j}^{k}\right),
\end{aligned}
$$

where $\alpha_{k}^{(q)}\left(\mathbf{x}_{i}^{k}, \mathbf{x}_{j}^{k}\right)$ is nonnegative weight to measure the importance of the $q$-th local metric to both $\mathrm{x}_{i}^{k}$ and $\mathrm{x}_{j}^{k}$, which ensures the learned dissimilarity function of $\mathrm{x}_{i}^{k}$ and $\mathrm{x}_{j}^{k}$ to be 
local. In addition, a global metric $\mathbf{M}_{k}^{(0)}$ with a positive constant weight $\alpha_{k}^{(0)}\left(\mathbf{x}_{i}^{k}, \mathbf{x}_{j}^{k}\right)=c_{k}$ is complemented into (5.21) to handle the part of the dissimilarity function shared by the whole input space. Thus, the final distance (or dissimilarity function) of a pair $\mathbf{x}_{i}^{k}$ and $\mathbf{x}_{j}^{k}$ is given as:

$$
\begin{aligned}
d_{\mathbf{M}_{k}}^{2}\left(\mathbf{x}_{i}^{k}, \mathbf{x}_{j}^{k}\right) & =\left(\mathbf{x}_{i}^{k}-\mathbf{x}_{j}^{k}\right)^{T}\left(\sum_{q=0}^{Q_{k}} \alpha_{k}^{(q)}\left(\mathbf{x}_{i}^{k}, \mathbf{x}_{j}^{k}\right) \mathbf{M}_{k}^{(q)}\right)\left(\mathbf{x}_{i}^{k}-\mathbf{x}_{j}^{k}\right) \\
& =\left(\mathbf{x}_{i}^{k}-\mathbf{x}_{j}^{k}\right)^{T} \mathbf{M}_{k}\left(\mathbf{x}_{i}^{k}, \mathbf{x}_{j}^{k}\right)\left(\mathbf{x}_{i}^{k}-\mathbf{x}_{j}^{k}\right)
\end{aligned}
$$

where the PSD matrix $\mathbf{M}_{k}(\cdot, \cdot) \in \mathbb{R}^{d_{k} \times d_{k}}$ is a matrix-valued function, and it is weighted by $Q_{k}+1$ matrices for a sample pair $\mathbf{x}_{i}^{k}$ and $\mathbf{x}_{j}^{k}$ as follows:

$$
\mathbf{M}_{k}\left(\mathbf{x}_{i}^{k}, \mathbf{x}_{j}^{k}\right)=\left(\sum_{q=0}^{Q_{k}} \alpha_{k}^{(q)}\left(\mathbf{x}_{i}^{k}, \mathbf{x}_{j}^{k}\right) \mathbf{M}_{k}^{(q)}\right)
$$

Furthermore, the weight $\alpha_{k}^{(q)}\left(\mathbf{x}_{i}^{k}, \mathbf{x}_{j}^{k}\right)$ in the local distance function (5.23) is defined as:

$$
\alpha_{k}^{(q)}\left(\mathbf{x}_{i}^{k}, \mathbf{x}_{j}^{k}\right)=\left\{\begin{array}{ll}
c_{k} & \text { if } q=0 \\
u_{k}^{(q)}\left(\mathbf{x}_{i}^{k}\right) u_{k}^{(q)}\left(\mathbf{x}_{j}^{k}\right) & \text { otherwise }
\end{array},\right.
$$

in which $u_{k}^{(q)}\left(\mathbf{x}_{i}^{k}\right)$ is a gating model to assign weight to the $q$-th local distance metric in a data-dependent way. In our experiments, we adopt a softmax gating function [137], which is given as follows:

$$
u_{k}^{(q)}\left(\mathbf{x}_{i}^{k}\right)=\frac{\exp \left(\mathbf{v}_{k}^{(q)^{T}} \mathbf{x}_{i}^{k}+b_{k}^{(q)}\right)}{\sum_{m=1}^{Q_{k}} \exp \left(\mathbf{v}_{k}^{(m)^{T}} \mathbf{x}_{i}^{k}+b_{k}^{(m)}\right)}
$$

for the $q$-th local metric, where $\mathbf{v}_{k}^{(q)}$ and $b_{k}^{(q)}$ are the weighting and bias parameters of this gating function respectively, and we have $u_{k}^{(q)}\left(\mathbf{x}_{i}^{k}\right) \geq 0$ for $1 \leq q \leq Q_{k}$ and $1 \leq k \leq K$. This gating function parametrized by the parameters $\left\{\mathbf{v}_{k}^{(q)}, b_{k}^{(q)}\right\}_{q=1}^{Q_{k}}$ is used to compute the weight (or probability) that an input vector $\mathrm{x}_{i}^{k}$ belongs to each local distance metric 
space. For example, a face image can be assigned to multiple metric spaces (e.g., age, expression, gender, race, etc.) with different weights. After having learned the parameters $\left\{\mathbf{v}_{k}^{(q)}, b_{k}^{(q)}\right\}_{q=1}^{Q_{k}}$ on the training data, this softmax gating function can be easily applied to other input vectors in a data-dependent way.

Then, we formulate our local metric learning (LML) method with respect to the single feature type $k$ under the same large margin framework as used in (5.3):

$$
\min _{\left\{\mathbf{M}_{k}^{(q)}\right\}_{q=0}^{Q_{k}},\left\{\mathbf{v}_{k}^{(q)}, b_{k}^{(q)}\right\}_{q=1}^{Q_{k}}} J_{k}=\sum_{i, j} h\left(\tau_{k}-y_{i j}\left(\mu_{k}-d_{\mathbf{M}_{k}}^{2}\left(\mathbf{x}_{i}^{k}, \mathbf{x}_{j}^{k}\right)\right)\right) .
$$

The objective function (5.26) is not jointly convex to $\left\{\mathbf{M}_{k}^{(q)}\right\}_{q=0}^{Q_{k}}$ and $\left\{\mathbf{v}_{k}^{(q)}, b_{k}^{(q)}\right\}_{q=1}^{Q_{k}}$, and it is non-trivial to find a global solution. To obtain these parameters, we employ the alternating optimization strategy and the gradient descent based method to achieve the local optimal solution.

Step 1: Fix $\left\{\mathbf{M}_{k}^{(r)}\right\}_{r=0}^{Q_{k}} \backslash \mathbf{M}_{k}^{(q)}$ and $\left\{\mathbf{v}_{k}^{(r)}, b_{k}^{(r)}\right\}_{r=1}^{Q_{k}}$, Update $\mathbf{M}_{k}^{(q)}$ The partial derivative of $J_{k}$ with regard to $\mathbf{M}_{k}^{(q)}, 0 \leq q \leq Q_{k}$, can be calculated by:

$$
\frac{\partial J_{k}}{\partial \mathbf{M}_{k}^{(q)}}=\sum_{i, j} y_{i j} h^{\prime}\left(z_{i j}\right) \alpha_{k}^{(q)}\left(\mathbf{x}_{i}^{k}, \mathbf{x}_{j}^{k}\right) \mathbf{C}_{i j}^{k}
$$

where $z_{i j}=\tau_{k}-y_{i j}\left(\mu_{k}-d_{\mathbf{M}_{k}}^{2}\left(\mathbf{x}_{i}^{k}, \mathbf{x}_{j}^{k}\right)\right)$, and $\mathbf{C}_{i j}^{k}$ is the outer product of pairwise differences given by (5.9).

Step 2: Fix $\left\{\mathbf{M}_{k}^{(r)}\right\}_{r=0}^{Q_{k}}$ and $\left\{\mathbf{v}_{k}^{(r)}, b_{k}^{(r)}\right\}_{r=1}^{Q_{k}} \backslash\left\{\mathbf{v}_{k}^{(q)}, b_{k}^{(q)}\right\}$, Update $\mathbf{v}_{k}^{(q)}$ and $b_{k}^{(q)}$ The partial derivative of $J_{k}$ with regard to $\mathbf{v}_{k}^{(q)}$ and $b_{k}^{(q)}$ can be computed by:

$$
\begin{aligned}
\frac{\partial J_{k}}{\partial \mathbf{v}_{k}^{(q)}} & =\sum_{i, j} y_{i j} h^{\prime}\left(z_{i j}\right) \sum_{m=1}^{Q_{k}} u_{k}^{(m)}\left(\mathbf{x}_{i}^{k}\right) u_{k}^{(m)}\left(\mathbf{x}_{j}^{k}\right) d_{\mathbf{M}_{k}^{(m)}}^{2}\left(\mathbf{x}_{i}^{k}, \mathbf{x}_{j}^{k}\right) \\
& \times\left(\left[\delta(q-m)-u_{k}^{(q)}\left(\mathbf{x}_{i}^{k}\right)\right] \mathbf{x}_{i}^{k}+\left[\delta(q-m)-u_{k}^{(q)}\left(\mathbf{x}_{j}^{k}\right)\right] \mathbf{x}_{j}^{k}\right)
\end{aligned}
$$




$$
\begin{aligned}
\frac{\partial J_{k}}{\partial b_{k}^{(q)}} & =\sum_{i, j} y_{i j} h^{\prime}\left(z_{i j}\right) \sum_{m=1}^{Q_{k}} u_{k}^{(m)}\left(\mathbf{x}_{i}^{k}\right) u_{k}^{(m)}\left(\mathbf{x}_{j}^{k}\right) d_{\mathbf{M}_{k}^{(m)}}^{2}\left(\mathbf{x}_{i}^{k}, \mathbf{x}_{j}^{k}\right) \\
& \times\left(\delta(q-m)-u_{k}^{(q)}\left(\mathbf{x}_{i}^{k}\right)+\delta(q-m)-u_{k}^{(q)}\left(\mathbf{x}_{j}^{k}\right)\right)
\end{aligned}
$$

in which the delta function $\delta(q-m)=1$ if $q=m$ and 0 otherwise for $q=1,2, \cdots, Q_{k}$.

We repeat the above two steps until the algorithm reaches certain convergence conditions. Moreover, we also decompose $\mathbf{M}_{k}^{(q)}$ into $\mathbf{M}_{k}^{(q)}=\mathbf{L}_{k}^{(q)^{T}} \mathbf{L}_{k}^{(q)}$ in the objective function (5.26) to reduce the number of parameters in optimization.

\subsubsection{Local Large-Margin Multi-Metric Learning}

The local metric learning (LML) method only learns a set of local distance metrics for each type of feature representation such that it cannot exploit discriminative information of other types of features. To utilize multiple features, we propose a local large-margin multimetric learning $\left(\mathrm{L}^{2} \mathrm{M}^{3} \mathrm{~L}\right)$ method by integrating the local metric learning (LML) (5.26) and the large-margin multi-metric learning $\left(\mathrm{LM}^{3} \mathrm{~L}\right)$ (5.6) into a unified framework. The objective function of the $\mathrm{L}^{2} \mathrm{M}^{3} \mathrm{~L}$ is formulated as:

$$
\begin{gathered}
\min _{\left\{\left\{\mathbf{M}_{k}^{(q)}\right\}_{q=0}^{Q_{k}},\left\{\mathbf{v}_{k}^{(q)}, b_{k}^{(q)}\right\}_{q=1}^{Q_{k}}, w_{k}\right\}_{k=1}^{K}} J=\sum_{k=1}^{K} w_{k}^{p} J_{k}+\lambda \sum_{\substack{k, \ell=1 \\
k<\ell}}^{K} \sum_{i, j}\left(d_{\mathbf{M}_{k}}\left(\mathbf{x}_{i}^{k}, \mathbf{x}_{j}^{k}\right)-d_{\mathbf{M}_{\ell}}\left(\mathbf{x}_{i}^{\ell}, \mathbf{x}_{j}^{\ell}\right)\right)^{2} \\
\text { s.t. } \quad \sum_{k=1}^{K} w_{k}=1, w_{k} \geq 0, \lambda>0
\end{gathered}
$$

It is obviously that the $\mathrm{LM}^{3} \mathrm{~L}$ is a special case of the $\mathrm{L}^{2} \mathrm{M}^{3} \mathrm{~L}$ method where only several global distance metrics are jointly solved. To minimize the optimization problem (5.30), we adopt similar methods as used in both $\mathrm{LM}^{3} \mathrm{~L}$ and LML.

Step 1: Fix $\left\{\left\{\mathbf{M}_{k}^{(r)}\right\}_{r=0}^{Q_{k}},\left\{\mathbf{v}_{k}^{(r)}, b_{k}^{(r)}\right\}_{r=1}^{Q_{k}}, w_{k}\right\}_{k=1}^{K} \backslash \mathbf{M}_{k}^{(q)}$, Update $\mathbf{M}_{k}^{(q)} \quad$ We update $\mathbf{M}_{k}^{(q)}$, $1 \leq k \leq K, 0 \leq q \leq Q_{k}$, by fixing other parameters. The partial derivative of $J$ with 
regard to $\mathbf{M}_{k}^{(q)}$ can be calculated by:

$$
\begin{aligned}
\frac{\partial J}{\partial \mathbf{M}_{k}^{(q)}} & =w_{k}^{p} \sum_{i, j} y_{i j} h^{\prime}\left(z_{i j}\right) \alpha_{k}^{(q)}\left(\mathbf{x}_{i}^{k}, \mathbf{x}_{j}^{k}\right) \mathbf{C}_{i j}^{k} \\
& +\lambda \sum_{\substack{\ell=1 \\
\ell \neq k}}^{K} \sum_{i, j}\left(1-\frac{d_{\mathbf{M}_{\ell}}\left(\mathbf{x}_{i}^{\ell}, \mathbf{x}_{j}^{\ell}\right)}{d_{\mathbf{M}_{k}}\left(\mathbf{x}_{i}^{k}, \mathbf{x}_{j}^{k}\right)}\right) \alpha_{k}^{(q)}\left(\mathbf{x}_{i}^{k}, \mathbf{x}_{j}^{k}\right) \mathbf{C}_{i j}^{k},
\end{aligned}
$$

where $z_{i j}=\tau_{k}-y_{i j}\left(\mu_{k}-d_{\mathbf{M}_{k}}^{2}\left(\mathbf{x}_{i}^{k}, \mathbf{x}_{j}^{k}\right)\right)$, and $\mathbf{C}_{i j}^{k}$ is the outer product of pairwise differences given by (5.9).

Step 2: Fix $\left\{\left\{\mathbf{M}_{k}^{(r)}\right\}_{r=0}^{Q_{k}},\left\{\mathbf{v}_{k}^{(r)}, b_{k}^{(r)}\right\}_{r=1}^{Q_{k}}, w_{k}\right\}_{k=1}^{K} \backslash\left\{\mathbf{v}_{k}^{(q)}, b_{k}^{(q)}\right\}$, Update $\mathbf{v}_{k}^{(q)}$ and $b_{k}^{(q)}$ The partial derivative of $J$ with respect to $\mathbf{v}_{k}^{(q)}$ and $b_{k}^{(q)}, 1 \leq k \leq K, 1 \leq q \leq Q_{k}$, can be computed by (5.32) and (5.33) respectively, where $z_{i j}=\tau_{k}-y_{i j}\left(\mu_{k}-d_{\mathbf{M}_{k}}^{2}\left(\mathbf{x}_{i}^{k}, \mathbf{x}_{j}^{k}\right)\right)$.

Step 3: Fix $\left\{\left\{\mathbf{M}_{k}^{(r)}\right\}_{r=0}^{Q_{k}},\left\{\mathbf{v}_{k}^{(r)}, b_{k}^{(r)}\right\}_{r=1}^{Q_{k}}\right\}_{k=1}^{K}$, Update $\left\{w_{k}\right\}_{k=1}^{K} \quad$ Following the same procedures as in $\mathrm{LM}^{3} \mathrm{~L}$, we update $w_{k}$ by utilizing the method of Lagrange multipliers. In this fashion, the closed-form solution can be obtained, and the $w_{k}, 1 \leq k \leq K$, is given by:

$$
w_{k}=\frac{\left(1 / J_{k}\right)^{1 /(p-1)}}{\sum_{k=1}^{K}\left(1 / J_{k}\right)^{1 /(p-1)}} .
$$

Then, we alternately update above three steps until the proposed $\mathrm{L}^{2} \mathrm{M}^{3} \mathrm{~L}$ method reaches a certain convergence condition, and then we will find the optimal solution, $\left\{\mathbf{M}_{k}^{(q)}\right\}_{q=0}^{Q_{k}}$, $\left\{\mathbf{v}_{k}^{(q)}, b_{k}^{(q)}\right\}_{q=1}^{Q_{k}}, w_{k}, k=1,2, \cdots, K$, of the $\mathrm{L}^{2} \mathrm{M}^{3} \mathrm{~L}$ method.

Algorithm 4 lists the main steps of the $\mathrm{L}^{2} \mathrm{M}^{3} \mathrm{~L}$ method.

After obtaining the multiple local and global distance metrics: $\left\{\mathbf{M}_{k}^{(q)}\right\}_{q=0}^{Q_{k}}$, gating model: $\left\{\mathbf{v}_{k}^{(q)}, b_{k}^{(q)}\right\}_{q=1}^{Q_{k}}$, and weight $w_{k}$ for all the $k=1,2, \cdots, K$, the distance of two multifeature data points $\mathbf{x}_{i}$ and $\mathbf{x}_{j}$ under the learned local metrics by $\mathrm{L}^{2} \mathrm{M}^{3} \mathrm{~L}$ can be calculated 
원

\%

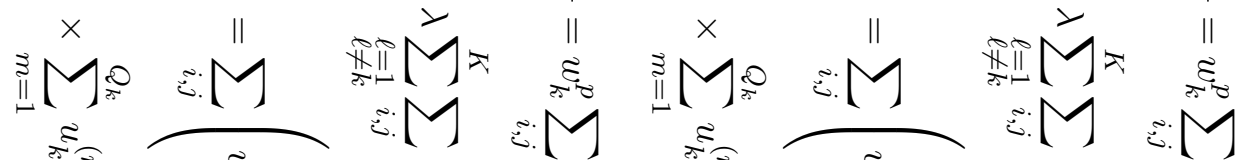

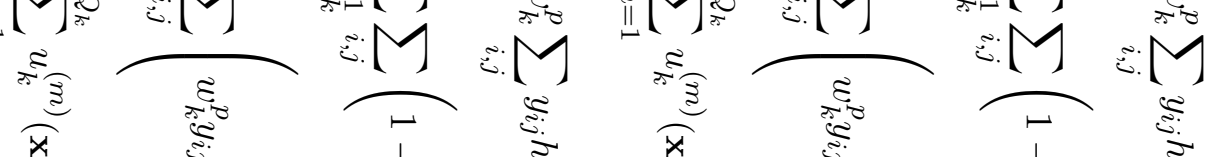

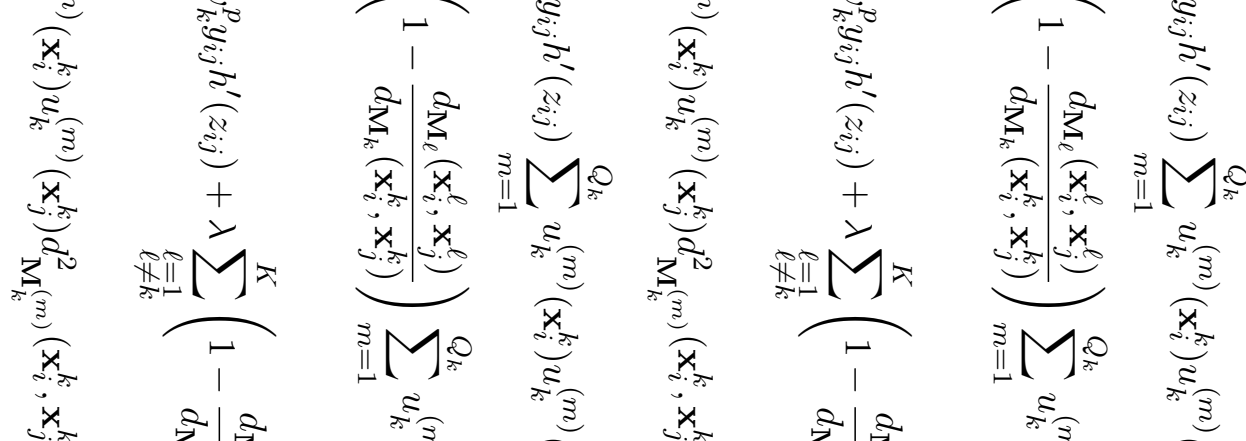

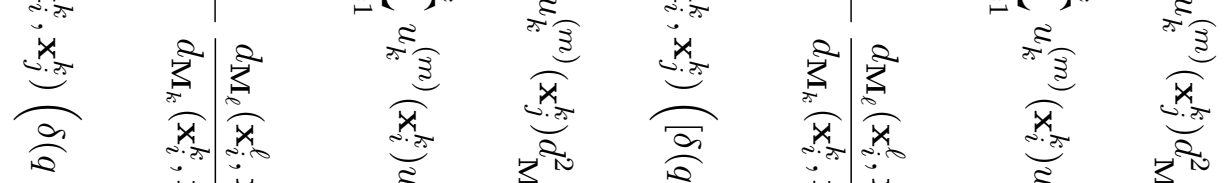

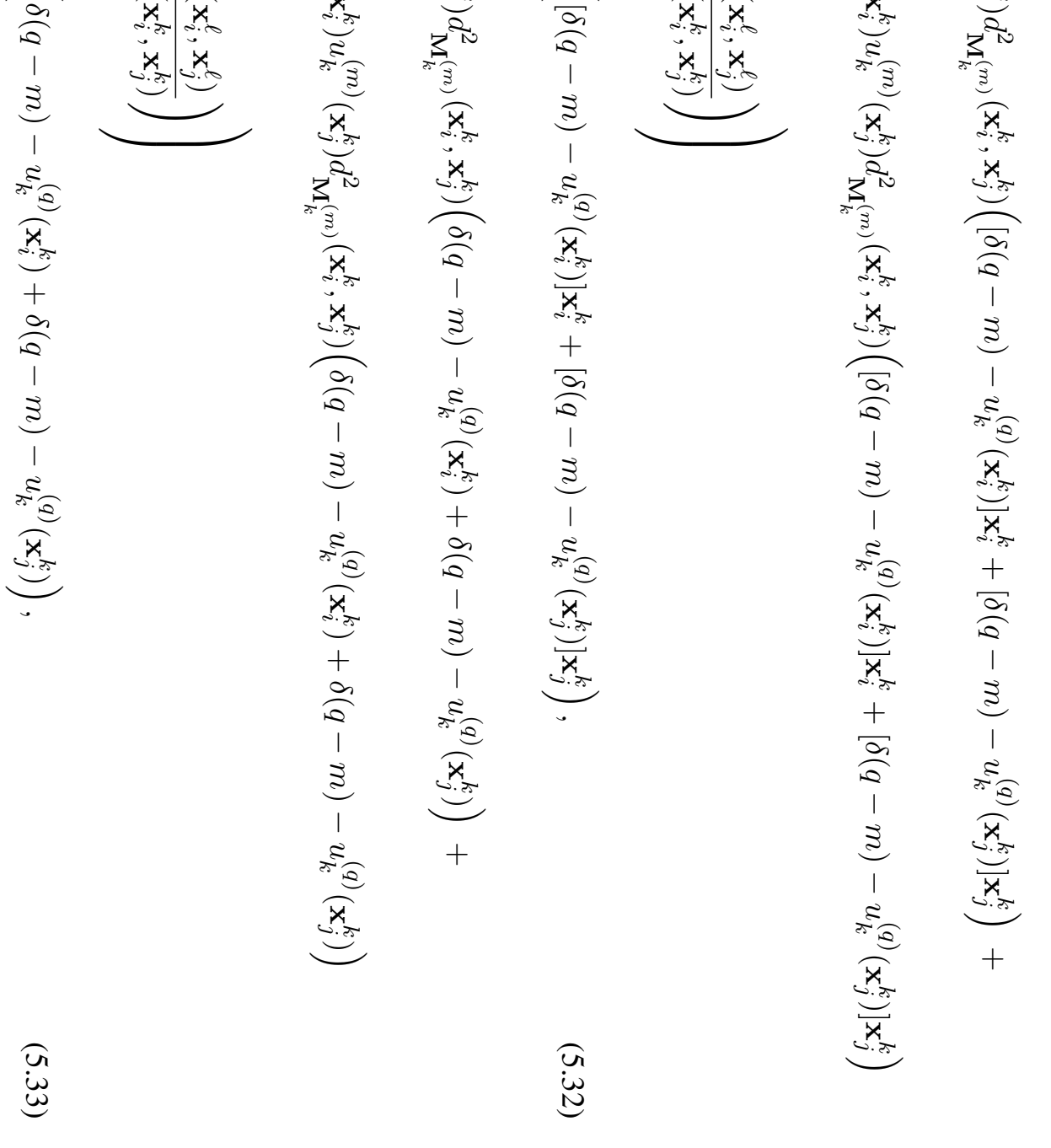




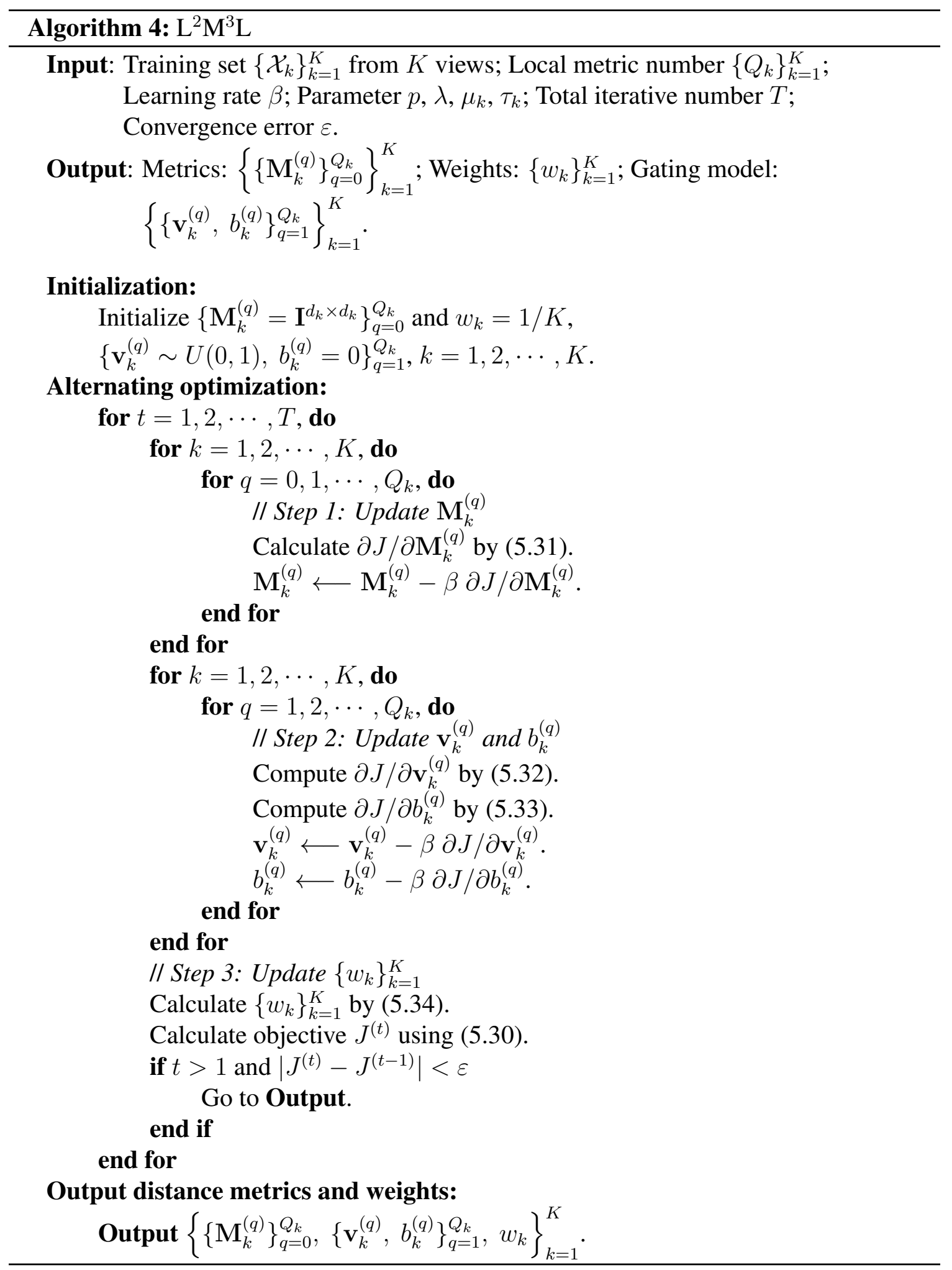


as follows:

$$
\begin{aligned}
d_{\mathrm{L}^{2} \mathrm{M}^{3} \mathrm{~L}}^{2}\left(\mathbf{x}_{i}, \mathbf{x}_{j}\right) & =\sum_{k=1}^{K} w_{k}\left(\mathbf{x}_{i}^{k}-\mathbf{x}_{j}^{k}\right)^{T}\left(\sum_{q=0}^{Q_{k}} \alpha_{k}^{(q)}\left(\mathbf{x}_{i}^{k}, \mathbf{x}_{j}^{k}\right) \mathbf{M}_{k}^{(q)}\right)\left(\mathbf{x}_{i}^{k}-\mathbf{x}_{j}^{k}\right) \\
& =\sum_{k=1}^{K} w_{k}\left(\mathbf{x}_{i}^{k}-\mathbf{x}_{j}^{k}\right)^{T} \mathbf{M}_{k}\left(\mathbf{x}_{i}^{k}, \mathbf{x}_{j}^{k}\right)\left(\mathbf{x}_{i}^{k}-\mathbf{x}_{j}^{k}\right) \\
& =\sum_{k=1}^{K} w_{k} d_{\mathbf{M}_{k}}^{2}\left(\mathbf{x}_{i}^{k}, \mathbf{x}_{j}^{k}\right) .
\end{aligned}
$$

\subsection{Experiments}

To evaluate the effectiveness of the proposed $\mathrm{LM}^{3} \mathrm{~L}, \mathrm{LML}$ and $\mathrm{L}^{2} \mathrm{M}^{3} \mathrm{~L}$ methods, we conducted face verification and kinship verification under unconstrained conditions on three real-world face datasets, i.e., the Labeled Faces in the Wild (LFW) [69], the YouTube Faces (YTF) [74], and the KinFaceW-II [83]. Figure 5.2 shows some samples of face images from these three datasets.

Baseline: We evaluated the proposed methods with three baseline methods using different metric learning strategies:

- Single Metric Learning (SML): we learned a single distance metric by using objective function (5.3) with single-view feature representation;

- Concatenated Metric Learning (CML): we first concatenated different feature representations into a long feature vector and then employed objective function (5.3) to learn a distance metric;

- Individual Metric Learning (IML): we learned the distance metric for each feature representation under the objective function (5.3) and then adopted the same weight (i.e., $\left.w_{k}=1 / K\right)$ to calculate the dissimilarity between a pair of face images by (5.5). 

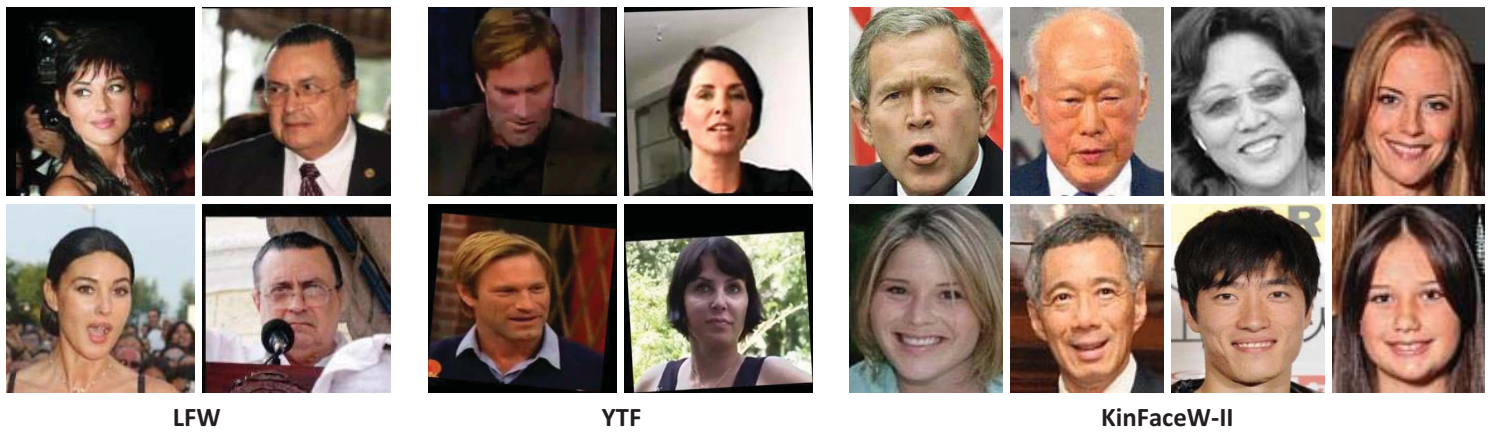

Figure 5.2: Some sample positive pairs from the LFW, YTF and KinFaceW-II datasets, where each column shows two face images of a positive pair.

In addition, several free parameters $p, \beta, \lambda, \mu_{k}, \tau_{k}, Q_{k}$ and $c_{k}$ of our $\mathrm{LM}^{3} \mathrm{~L}, \mathrm{LM}-$ $\mathrm{L}$ and $\mathrm{L}^{2} \mathrm{M}^{3} \mathrm{~L}$ methods were empirically set as $2,0.001,0.1,5,1,3$ and 1 for all the $k=1,2, \cdots, K$ respectively, unless stated otherwise. The following subsections detail the experimental settings and results on three datasets.

\subsubsection{Image-based Face Verification}

\subsubsection{Dataset and Settings}

The LFW dataset [69] contains more than 13000 face images of 5749 subjects collected from the web with large variations in expression, pose, age, illumination, resolution, etc. There are two training paradigms for supervised learning on this dataset: image restricted and image unrestricted. In our experiments, we used the image restricted setting where only the pairwise label information is provided to train our methods. We followed the standard evaluation protocol on the "View 2" dataset [69] which consists of 3000 matched pairs (or positive pairs) and 3000 mismatched pairs (or negative pairs), and all these pairs were divided into 10 folds and each fold contains 300 positive pairs and 300 negative pairs of face iamges. We used the LFW-a dataset ${ }^{1}$ for our experimental evaluation, and thus our setting on this dataset falls into the category of image-restricted, label-free outside data.

\footnotetext{
${ }^{1}$ http://www.openu.ac.il/home/hassner/data/lfwa/.
} 
For each face image, we first cropped it into $80 \times 150$ pixels from its center to remove the background information, and then extracted three types of feature representations:

- Dense SIFT (DSIFT) [78]: Firstly, we densely sampled SIFT descriptors on each $16 \times 16$ patch without overlapping and obtained 45 SIFT descriptors. Then, we concatenated these SIFT descriptors to form a 5760-dimensional feature vector;

- LBP [79]: We divided each image into $8 \times 15$ non-overlapping blocks, where the size of each block is $10 \times 10$. Then, we extracted a 59-dimensional uniform pattern LBP feature for each block and concatenated them to form a 7080-dimensional feature vector;

- Sparse SIFT (SSIFT): We used the SSIFT feature provided by [7], which first localized nine fixed landmarks in each image and extracted SIFT descriptors over three scales at these landmarks, and then concatenated these 27 SIFT descriptors to result in a 3456-dimensional feature vector.

For these three kinds of features, we employed whitened PCA (WPCA) to project each feature vector into a 200-dimensional feature subspace, respectively. Note that we first employed the WPCA on the training set to compute the projection matrix, and then we used this projection matrix to reduce the dimension of each sample in the training set and testing set.

\subsubsection{Comparison with Baseline Methods}

Table 5.1 records the verification accuracy with standard error of our methods and baseline methods by different metric learning strategies on the LFW dataset under category of image-restricted, label-free outside data. We see that the $\mathrm{LM}^{3} \mathrm{~L}$ and $\mathrm{L}^{2} \mathrm{M}^{3} \mathrm{~L}$ methods consistently outperforms these baseline methods in terms of the mean verification accuracy. 
Table 5.1: Comparisons of the mean verification accuracy (\%) with several baseline methods on the LFW under category of image-restricted, label-free outside data.

\begin{tabular}{|l|c|c|}
\hline Method & Feature & Accuracy (\%) \\
\hline \hline SML & DSIFT & $84.30 \pm 0.69$ \\
SML & LBP & $83.83 \pm 0.41$ \\
SML & SSIFT & $84.58 \pm 0.36$ \\
CML & All & $82.40 \pm 0.51$ \\
IML & All & $87.78 \pm 0.58$ \\
\hline \hline LML & DSIFT & $86.33 \pm 0.66$ \\
LML & LBP & $85.98 \pm 0.44$ \\
LML & SSIFT & $86.75 \pm 0.34$ \\
LM $^{3} \mathrm{~L}$ & All & $89.57 \pm 0.48$ \\
L $^{2} \mathbf{M}^{3} \mathrm{~L}$ & All & $\mathbf{9 0 . 2 3} \pm \mathbf{0 . 5 5}$ \\
\hline
\end{tabular}

Compared with SML (or LML), the $\mathrm{LM}^{3} \mathrm{~L}$ (or $\mathrm{L}^{2} \mathrm{M}^{3} \mathrm{~L}$ ) learns multiple distance metrics with multi-feature representations, such that more discriminative information can be exploited for verification. Compared with CML and IML, our $\mathrm{LM}^{3} \mathrm{~L}$ and $\mathrm{L}^{2} \mathrm{M}^{3} \mathrm{~L}$ jointly learn multiple distance metrics so that the distance metrics learned for different features can interact with each other, therefore more complementary information can be extracted for face verification. We also observe that local metric leaning methods (i.e., $L M L$ and $\mathrm{L}^{2} \mathrm{M}^{3} \mathrm{~L}$ ) obtain the better performance than their global companions (i.e., SML and $\mathrm{LM}^{3} \mathrm{~L}$ ). These results show that the $\mathrm{LML}$ and $\mathrm{L}^{2} \mathrm{M}^{3} \mathrm{~L}$ can exploit local specificities of data to improve performance of face verification.

\subsubsection{Comparison with State-of-the-Art Methods}

We also compared our $\mathrm{LM}^{3} \mathrm{~L}$ and $\mathrm{L}^{2} \mathrm{M}^{3} \mathrm{~L}$ methods with several state-of-the-art methods on the LFW dataset ${ }^{2}$. These methods can be categorized into metric learning based methods containing PCCA [97], DML-eig combined [103], CSML+SVM [8], SFRD+PMML [10],

\footnotetext{
${ }^{2}$ http://vis-www.cs.umass.edu/lfw/results.html.
} 


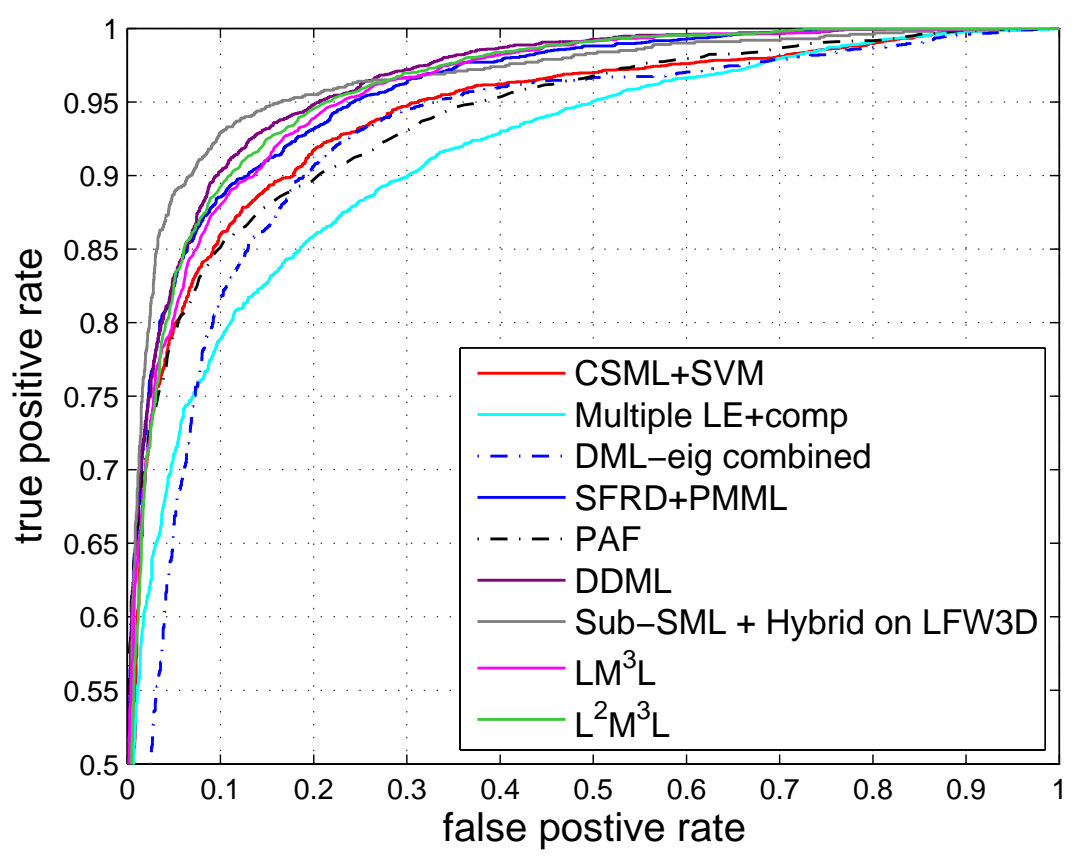

Figure 5.3: ROC curves of our methods and several state-of-the-art methods on the LFW under category of image-restricted, label-free outside data.

Sub-SML [104], large margin local metric learning (LMLML) [138], and discriminative deep metric learning (DDML) [11]; and descriptor based methods including LARK [139], pose adaptive filter (PAF) [72], high dimensional vector multiplication (VMRS) [105], Hybrid on LFW3D [140], and Spartans [141]. Table 5.2 tabulates the mean verification accuracy with standard error of different methods and Figure 5.3 shows ROC curves of several state-of-the-art methods on this dataset. We see that the proposed $\mathrm{LM}^{3} \mathrm{~L}$ and $\mathrm{L}^{2} \mathrm{M}^{3} \mathrm{~L}$ methods achieve competitive results compared with these state-of-the-art methods except two methods: Sub-SML + Hybrid on LFW3D [140] and HPEN + HD-LBP + DDML [142]. The reason is that they both employed the powerful face alignment techniques, and Sub-SML + Hybrid on LFW3D [140] adopted more than 10 types of features and HPEN + HD-LBP + DDML [142] exploited the over-complete high-dimensional feature (i.e., 100K-dim HDLBP) for face verification. 
Table 5.2: Comparisons of the mean verification accuracy (\%) with state-of-the-art results on the LFW under category of image-restricted, label-free outside data, where NoF denotes the number of feature used in each method.

\begin{tabular}{|l|c|c|}
\hline Method & NoF & Accuracy (\%) \\
\hline \hline PCCA [97] & 1 & $83.80 \pm 0.40$ \\
LARK supervised, aligned [139] & 1 & $85.10 \pm 0.59$ \\
Hybrid on LFW3D [140] & 12 & $85.63 \pm 0.53$ \\
DML-eig combined [103] & 8 & $85.65 \pm 0.56$ \\
LMLML [138] & 1 & $86.13 \pm 0.53$ \\
PAF [72] & 1 & $87.77 \pm 0.51$ \\
CSML+SVM [8] & 6 & $88.00 \pm 0.37$ \\
SFRD+PMML [10] & 8 & $89.35 \pm 0.50$ \\
Spartans [141] & 1 & $89.69 \pm 0.36$ \\
Sub-SML [104] & 6 & $89.73 \pm 0.38$ \\
TSML with feature fusion [143] & 12 & $89.80 \pm 0.47$ \\
DDML [11] & 6 & $90.68 \pm 1.41$ \\
VMRS [105] & 10 & $91.10 \pm 0.59$ \\
Sub-SML + Hybrid on LFW3D [140] & 12 & $91.65 \pm 1.04$ \\
HPEN + HD-LBP + DDML [142] & 1 & $92.57 \pm 0.36$ \\
\hline \hline LM ${ }^{3}$ L & 3 & $89.57 \pm 0.48$ \\
L $^{2}{ }^{3}$ L & 3 & $\mathbf{9 0 . 2 3} \pm \mathbf{0 . 5 5}$ \\
\hline
\end{tabular}

The reason that VMRS [105] outperforms $\mathrm{L}^{2} \mathrm{M}^{3} \mathrm{~L}$ is that: 1) VMRS adopted 10 different features (i.e., LBP, TPLBP, OCLBP, SIFT, Scattering, and their "square root" versions); 2) VMRS used high-dimensional features, e.g., the 40887-dimensional OCLBP, and the 96520-dimensional Scattering feature; and 3) VMRS combined the non-linear dimensionality reduction technique called Diffusion Maps (DM) [105] and WPCA to obtain an additional improvement in accuracy. In our methods, we used three low-dimensional features, where the WPCA is used to reduce the dimensionality. The reason that the performance of DDML [11] outperforms $\mathrm{L}^{2} \mathrm{M}^{3} \mathrm{~L}$ is that 1) The DDML employs 6 different features; and 2) The DDML adopts a nonlinear distance metric learning method via the neural network 
to exploit the nonlinearity of data points. In our methods, we use three low-dimensional features to learn several linear distance metrics.

We also evaluated our $\mathrm{L}^{2} \mathrm{M}^{3} \mathrm{~L}$ method on the LFW3D [140] images ${ }^{3}$, where the face images in LFW dataset were frontalized and aligned by a more powerful face alignment technique [140]. Table 5.3 shows the performance of $\mathrm{L}^{2} \mathrm{M}^{3} \mathrm{~L}$ on the LFW3D images. From this table, we see that our $\mathrm{L}^{2} \mathrm{M}^{3} \mathrm{~L}$ outperforms 1) Sub-SML + Hybrid on LFW3D [140] on the LFW3D images, and 2) HPEN + HD-LBP + DDML [142] when the high-dimensional LBP (HD-LBP) feature was used.

\subsubsection{Comparison with Methods $[11,105,140,142]$ with the Same Feature Set}

Generally, it is difficult to make a fair comparison of these methods [105, 140,142] with the same feature set, because these methods have different experimental settings and make individual contributions to face recognition. In HPEN + HD-LBP + DDML [142], a Highfidelity Pose and Expression Normalization (HPEN) method with 3D Morphable Model (3DMM) was proposed to automatically generate a natural face image in frontal pose and neutral expression, and the high-dimensional LBP (HD-LBP) (100000 dimensions) was extracted on each aligned face image. In Sub-SML + Hybrid on LFW3D [140], the authors used single, unmodified, 3D surface as an approximation to the shape of all input faces in order to produce frontalized views of LFW images (i.e., LFW3D images), and they extracted features: LBP, TPLBP, FPLBP, OSS LBP, OSS TPLBP, OSS FPLBP, and their "square root" versions. The main contributions of these methods [140,142] are providing powerful face alignment methods. VMRS [105] extracted 10 different features: LBP, TPLBP, OCLBP, SIFT, Scattering, and their "square root" versions, where the original dimensions were 7080, 40887, 9216, 3456 and 96520 for the LBP, OCLBP, TPLBP, SIFT and Scattering, respectively. Moreover, VMRS combined the non-linear dimensionality reduction

\footnotetext{
${ }^{3}$ http://www.openu.ac.il/home/hassner/projects/frontalize/
} 


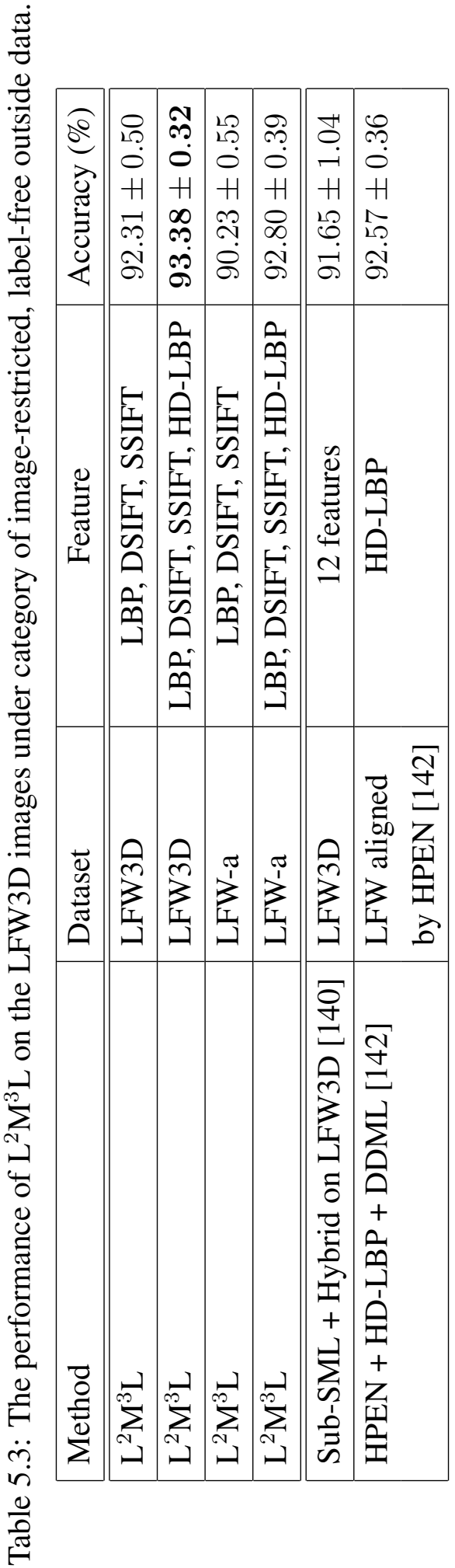


Table 5.4: Comparison of different methods using the same feature set on LFW dataset under category of image-restricted, label-free outside data.

\begin{tabular}{|l|c|c|}
\hline Method & Feature & Accuracy (\%) \\
\hline \hline VMRS [105] & & $88.15 \pm 0.61$ \\
DDML [11] & LBP, DSIFT, SSIFT & $90.10 \pm 0.52$ \\
L $^{2} \mathbf{M}^{3} \mathrm{~L}$ & & $90.23 \pm 0.55$ \\
\hline
\end{tabular}

technique called Diffusion Maps (DM) [105] and WPCA to obtain an additional improvement in terms of the accuracy.

We compared $\mathrm{L}^{2} \mathrm{M}^{3} \mathrm{~L}$ with VMRS [105] and DDML [11] with the same feature set (LBP, DSIFT, and SSIFT) on the LFW dataset. Table 5.4 reports the performance of three methods using the same feature set on LFW dataset under category of image-restricted, label-free outside data. We see that 1) $\mathrm{L}^{2} \mathrm{M}^{3} \mathrm{~L}$ outperforms the VMRS in terms of the mean accuracy, and 2) $\mathrm{L}^{2} \mathrm{M}^{3} \mathrm{~L}$ is comparable to DDML under the same feature set.

Given a pair of face images, the matching times of $\mathrm{L}^{2} \mathrm{M}^{3} \mathrm{~L}$, VMRS and DDML with the same feature set are about $0.02-0.03$ seconds on a standard Windows machine (Intel i53470 CPU @ 3.20 GHz, and 32 GB RAM) with the MATLAB code. From references [140, 142], we see that the matching times of methods in [142] and [140] are about 1.6 seconds and 0.3 seconds, and the face alignment procedure takes most of processing time.

\subsubsection{Comparison of $\mathbf{L} M^{3} \mathbf{L}$ and $\mathbf{L}^{2} \mathbf{M}^{3} \mathbf{L}$ with $\lambda=0$}

When $\lambda=0$, we evaluated the $\mathrm{LM}^{3} \mathrm{~L}(\lambda=0)$ and $\mathrm{L}^{2} \mathrm{M}^{3} \mathrm{~L}(\lambda=0)$ on LFW dataset under category of image-restricted, label-free outside data (see Table 5.5). Table 5.5 shows that the regularization term of the $\mathrm{LM}^{3} \mathrm{~L}$ and $\mathrm{L}^{2} \mathrm{M}^{3} \mathrm{~L}$ can help improve the verification accuracy. The reason is that the $\mathrm{LM}^{3} \mathrm{~L}$ and $\mathrm{L}^{2} \mathrm{M}^{3} \mathrm{~L}$ seek a commonality of multiple features and make all features more robust for face verification. 
Table 5.5: Comparison of $\mathrm{LM}^{3} \mathrm{~L}$ and $\mathrm{L}^{2} \mathrm{M}^{3} \mathrm{~L}$ when $\lambda=0$ on LFW dataset under category of image-restricted, label-free outside data.

\begin{tabular}{|l|c|c|}
\hline Method & Feature & Accuracy $(\%)$ \\
\hline \hline $\mathrm{LM}^{3} \mathrm{~L}(\lambda=0)$ & LBP, DSIFT, SSIFT & $88.02 \pm 0.56$ \\
$\mathrm{LM}^{3} \mathrm{~L}$ & & $89.57 \pm 0.48$ \\
\hline $\mathrm{L}^{2} \mathrm{M}^{3} \mathrm{~L}(\lambda=0)$ & LBP, DSIFT, SSIFT & $88.95 \pm 0.60$ \\
$\mathrm{~L}^{2} \mathrm{M}^{3} \mathrm{~L}$ & & $90.23 \pm 0.55$ \\
\hline
\end{tabular}

\subsubsection{Video-based Face Verification}

\subsubsection{Dataset and Settings}

The YTF dataset [74] consists of 3425 videos of 1595 different people collected from YouTube site. There are also large variations in pose, illumination, and expression in each video, and the average length of each video clip is 181.3 frames. In our experiments, we followed the standard evaluation protocol and evaluated our methods for unconstrained video-based face verification on the 5000 video pairs. These 5000 pairs are equally divided into 10 folds and each fold contains 250 intra-personal pairs (positive pairs) and 250 interpersonal pairs (negative pairs). We adopted the image restricted and image unrestricted protocols to evaluate the proposed methods. For the image restricted setting, we directly used three feature descriptors including LBP, Center-Symmetric LBP (CSLBP) [74] and Four-Patch LBP (FPLBP) [70] which are provided by [74]. Since all face images have been aligned by the detected facial key points, we simply averaged all the feature vectors within one video clip to result in a mean feature vector for each type of feature. Then, we employed WPCA to reduce each feature into a 200-dimensional feature vector.

\subsubsection{Comparison with Baseline Methods}

As in LFW dataset, we also compared our methods with several baseline methods using different metric learning strategies, i.e., SML, CML and IML on the YTF dataset under the 
Table 5.6: Comparison of the mean verification accuracy (\%) with baseline methods using different metric learning strategies on the YTF under the image restricted setting.

\begin{tabular}{|l|c|c|}
\hline Method & Feature & Accuracy (\%) \\
\hline \hline SML & CSLBP & $73.66 \pm 1.52$ \\
SML & FPLBP & $75.02 \pm 1.67$ \\
SML & LBP & $78.46 \pm 0.94$ \\
CML & All & $75.36 \pm 2.37$ \\
IML & All & $80.12 \pm 1.33$ \\
\hline \hline LML & CSLBP & $75.76 \pm 1.59$ \\
LML & FPLBP & $75.78 \pm 2.19$ \\
LML & LBP & $80.08 \pm 2.06$ \\
LM $^{3} \mathrm{~L}$ & All & $81.28 \pm 1.17$ \\
L $^{2} \mathbf{M}^{3} \mathrm{~L}$ & All & $\mathbf{8 1 . 7 2} \pm \mathbf{1 . 5 3}$ \\
\hline
\end{tabular}

image restricted setting. Table 5.6 records the mean verification accuracy with standard error of these metric learning methods on the YTF under the image restricted setting. We see that the proposed $\mathrm{LM}^{3} \mathrm{~L}$ and $\mathrm{L}^{2} \mathrm{M}^{3} \mathrm{~L}$ methods consistently perform better than these baseline methods in terms of the mean verification accuracy; and local based methods, LML and $\mathrm{L}^{2} \mathrm{M}^{3} \mathrm{~L}$, can make use of local structures of samples to enhance the verification accuracy. These two observations are in agreement with results obtained on the LFW dataset.

\subsubsection{Comparison with State-of-the-Art Methods}

We then compared $\mathrm{LM}^{3} \mathrm{~L}$ and $\mathrm{L}^{2} \mathrm{M}^{3} \mathrm{~L}$ with state-of-the-art methods on the YTF dataset ${ }^{4}$ under the image restricted setting. The compared methods include matched background similarity (MBGS) [74], APEM [110], STFRD+PMML [10], MBGS+SVM $\ominus[75], \mathrm{V}-$ SOF+OSS (Adaboost) [111], DDML [11], Eigen-PEP [144], deep mixture model and convolutional fusion network (DMM+CFN) [145], and LMKMML [117]. Table 5.7 lists the mean verification accuracy with the standard error, and Figure 5.4 shows ROC curves of

\footnotetext{
${ }^{4}$ http://www.cs.tau.ac.il/ wolf/ytfaces/results.html.
} 
Table 5.7: Comparisons of the mean verification accuracy with standard error (\%) with several state-of-the-art results on the YTF under the image restricted setting.

\begin{tabular}{|l|c|}
\hline Method & Accuracy (\%) \\
\hline \hline MBGS (LBP) [74] & $76.40 \pm 1.80$ \\
APEM (LBP) [110] & $77.44 \pm 1.46$ \\
APEM (fusion) [110] & $79.06 \pm 1.51$ \\
STFRD+PMML [10] & $79.48 \pm 2.52$ \\
MBGS+SVM $\ominus$ [75] & $78.90 \pm 1.90$ \\
VSOF+OSS (Adaboost) [111] & $79.70 \pm 1.80$ \\
DDML (combined) [11] & $82.34 \pm 1.47$ \\
LMKMML [117] & $82.70 \pm 1.50$ \\
DMM+CFN [145] & $82.80 \pm 0.90$ \\
Eigen-PEP [144] & $84.80 \pm 1.40$ \\
\hline \hline LM $^{3}$ L & $81.28 \pm 1.17$ \\
L $^{2} M^{3}$ L & $\mathbf{8 1 . 7 2} \pm \mathbf{1 . 5 3}$ \\
\hline
\end{tabular}

our methods and several state-of-the-art methods on the YTF dataset, respectively. We can observe that our $\mathrm{L}^{2} \mathrm{M}^{3} \mathrm{~L}$ method achieves competitive results compared with most of these state-of-the-art methods on this dataset under the image restricted setting. Additionally, the Eigen-PEP obtains the best accuracy, the reason is that it exploits intra-class variations between frames of each video clip while our implementation simply takes the mean of all frames for a video in feature representation.

\subsubsection{Comparison with Deep Learning based Methods}

We also evaluated our LML, $\mathrm{LM}^{3} \mathrm{~L}$ and $\mathrm{L}^{2} \mathrm{M}^{3} \mathrm{~L}$ methods using convolutional neural network $(\mathrm{CNN})$ feature which recently has achieved various promising results on face verification $[24,30,112,146]$. In our implementation, we employed the VGG-Face CNN model provided by [146] to compute CNN descriptor. Specifically, we only extracted CNN feature on the first 100 frames of each video at single scale. For each face image in YTF dataset, we first resized it to size of $200 \times 200$ pixels and cropped $100 \times 100$ region from its center, 


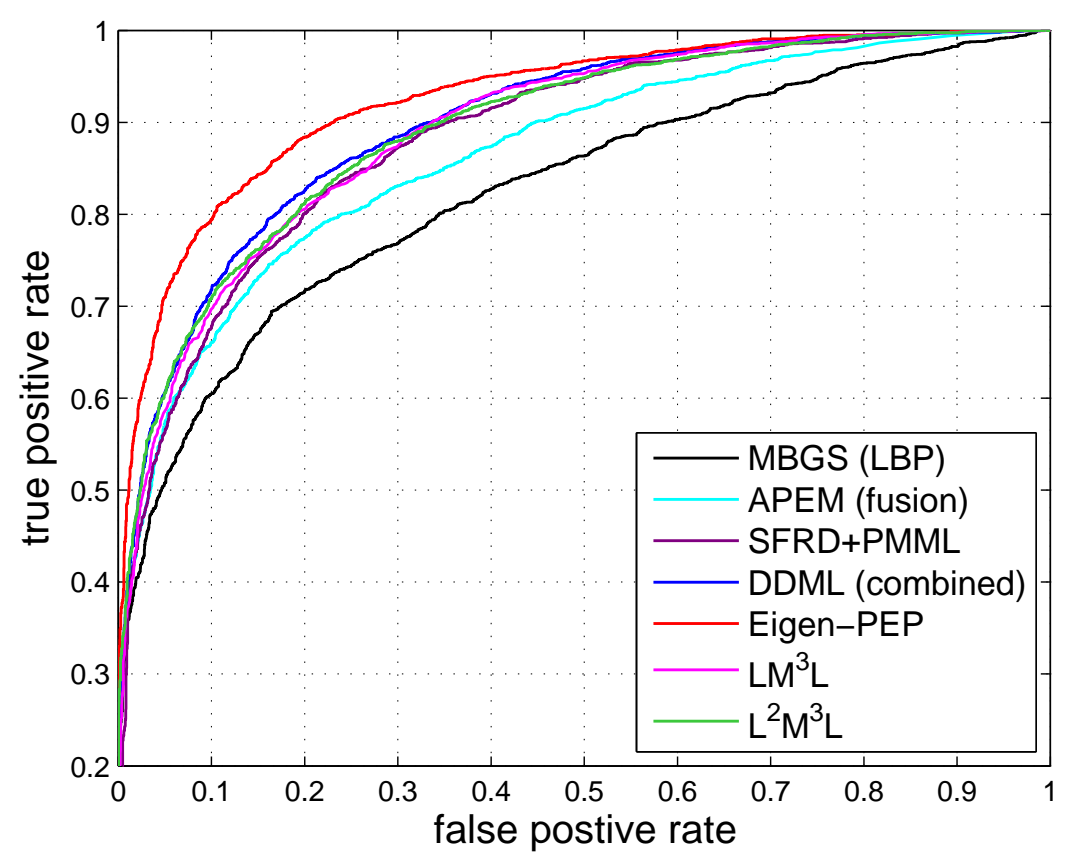

Figure 5.4: ROC curves of our methods and several state-of-the-art methods on the YTF under the image restricted setting.

then we resized it to $224 \times 224$ pixel image to compute 4096-dimensional CNN feature vector. Finally, we averaged these CNN feature vectors of the first 100 frames for each video, and each video was represented by a 4096-dimensional vector. Moreover, each feature vector was reduced to the size of 200 by PCA. Table 5.8 shows the mean verification accuracy of our proposed methods and several deep learning based methods using CNN feature (e.g., DeepFace [24], DeepID2+ [112], FaceNet [30], and VGG-Face CNN [146]) on the YTF dataset under the image unrestricted setting. We see that our $\mathrm{LM}^{3} \mathrm{~L}$ and $\mathrm{L}^{2} \mathrm{M}^{3} \mathrm{~L}$ methods can be comparable to the current state-of-the-art results on this dataset under the image unrestricted setting.

The Face Net [30] and Embedding loss [146] are two current state-of-the-art methods on YTF dataset. The reason that they outperformed $\mathrm{L}^{2} \mathrm{M}^{3} \mathrm{~L}$ is that:

- The Face Net [30] used about 200 million face images in the model training, and the 
Table 5.8: Comparison with deep learning based methods on the YTF dataset under image unrestricted setting.

\begin{tabular}{|l|c|c|}
\hline Method & Feature & Accuracy (\%) \\
\hline \hline SML & CNN & $93.92 \pm 1.18$ \\
LML & CNN & $94.56 \pm 1.24$ \\
LM $^{3}$ L & CNN, CSLBP, FPLBP, LBP & $94.75 \pm 1.21$ \\
L $^{2}{ }^{3}$ L & CNN, CSLBP, FPLBP, LBP & $\mathbf{9 4 . 9 0} \pm \mathbf{1 . 0 9}$ \\
\hline \hline DFD-SID+JB [147] & CNN & $89.10 \pm 0.40$ \\
DeepFace-single [24] & CNN & $91.4 \pm 1.1$ \\
DeepID2+ [112] & CNN & $93.2 \pm 0.2$ \\
FaceNet [30] & CNN & $95.12 \pm 0.39$ \\
Softmax (L2) [146] & CNN & 91.6 \\
Embedding loss [146] & CNN & 97.3 \\
\hline
\end{tabular}

VGG-Face CNN model used in our method used about 2.6 million face images.

- To extract CNN features for each face video, the Embedding loss [146] selected the top 100 frames of this video by ordering the faces by their facial landmark confidence score. In our $\mathrm{L}^{2} \mathrm{M}^{3} \mathrm{~L}$, we only took the first 100 frames by following the setting in the Face Net [30].

- The Face Net [30] and Embedding loss [146] are two strongly-supervised learning methods, because they employ the triplet loss function which exploits the label information of each face video of YTF dataset. Unlike these methods, our $\mathrm{L}^{2} \mathrm{M}^{3} \mathrm{~L}$ method is a weakly-supervised learning method which only exploits the pairwise supervision from face video pairs.

In Table 5.8, the main aim of $\mathrm{L}^{2} \mathrm{M}^{3} \mathrm{~L}$ and $\mathrm{LML}$ is to show that 1) our metric learning methods learn the favorable distance metrics to improve the performance of Softmax (L2) [30] (91.6\%) when the CNN feature is used; and 2) $\mathrm{L}^{2} \mathrm{M}^{3} \mathrm{~L}$ can further improve the verification accuracy by integrating low-level features and high-level features into a unified framework. 


\subsubsection{Kinship Verification}

\subsubsection{Dataset and Settings}

The KinFaceW-II [83] is a kinship face dataset collected from the public figures or celebrities and their parents or children. There are four kinship relations in the KinFaceWII datasets: Father-Son (F-S), Father-Daughter (F-D), Mother-Son (M-S) and MotherDaughter (M-D), and each relation includes 250 pairs of kinship images. Following the experimental settings in [83], we constructed 250 positive pairs (with kinship) and 250 negative pairs (without kinship) for each relation. For each face image, we extracted four types of feature representations as follows:

- LEarning-based descriptor (LE) [148]: Following the same parameter settings used in $[83,148]$, we first obtained 200 cluster centers by k-means clustering, and then performed vector quantization to obtain a 200-bin histogram feature for the whole face image;

- LBP: A 256-bin histogram feature was extracted;

- TPLBP [70]: We obtained a 256-bin histogram feature for each image by adopting the default setting in [70].

- SIFT: We densely sampled SIFT descriptors on $16 \times 16$ blocks with space of 8 pixels, and then computed a 200-bin histogram feature for each image by adopting the bagof-visual-words model [149].

We adopted the 5 -fold cross validation strategy for each of the four relations in this dataset and the final results were reported by the mean verification accuracy. 
Table 5.9: Comparisons of the mean verification accuracy (\%) with baseline methods using different metric learning strategies on the KinFaceW-II dataset.

\begin{tabular}{|l|c|c|c|c|c|c|}
\hline Method & Feature & F-S & F-D & M-S & M-D & Mean \\
\hline \hline SML & LE & 76.2 & 70.1 & 72.4 & 71.8 & 72.6 \\
SML & LBP & 66.9 & 65.5 & 63.1 & 68.3 & 66.0 \\
SML & TPLBP & 71.8 & 63.3 & 63.0 & 67.6 & 66.4 \\
SML & SIFT & 68.1 & 63.8 & 67.0 & 63.9 & 65.7 \\
CML & All & 76.3 & 67.5 & 74.3 & 75.4 & 73.4 \\
IML & All & 79.4 & 71.5 & 76.3 & 77.3 & 76.1 \\
\hline \hline LML & LE & 76.8 & 74.2 & 76.6 & 73.8 & 75.4 \\
LML & LBP & 66.0 & 64.8 & 67.8 & 66.8 & 66.4 \\
LML & TPLBP & 68.6 & 66.2 & 65.4 & 70.8 & 67.8 \\
LML $^{\text {SML }}$ & SIFT & 72.2 & 66.0 & 68.2 & 66.2 & 68.2 \\
LM $^{3}$ L & All & $\mathbf{8 2 . 4}$ & 74.2 & $\mathbf{7 9 . 6}$ & 78.7 & 78.7 \\
L $^{2}{ }^{3}$ L & All & $\mathbf{8 2 . 4}$ & $\mathbf{7 8 . 2}$ & 78.8 & $\mathbf{8 0 . 4}$ & $\mathbf{8 0 . 0}$ \\
\hline
\end{tabular}

\subsubsection{Comparison with Baseline Methods}

We first compared our method with SML, CML, and IML on the KinFaceW-II dataset. Table 5.9 records the mean verification accuracy of our methods and baseline methods using various metric learning strategies on the KinFaceW-II dataset for four kinship relations, respectively. We also see that the $\mathrm{LML}, \mathrm{LM}^{3} \mathrm{~L}$ and $\mathrm{L}^{2} \mathrm{M}^{3} \mathrm{~L}$ consistently outperforms baseline metric learning strategies on four relations in mean verification accuracy.

\subsubsection{Comparison with Multiple Metric Learning Methods}

We further compared the $\mathrm{LM}^{3} \mathrm{~L}$ and $\mathrm{L}^{2} \mathrm{M}^{3} \mathrm{~L}$ methods with several multiple metric learning methods for kinship verification. These multiple metric learning methods include multiple canonical correspondence analysis (MCCA) [135], multiple NRML (MNRML) [83], discriminative multimetric learning (DMML) [150], and PMML [10]. Table 5.10 reports the mean verification accuracy of our methods and these multiple metric learning methods. We 
Table 5.10: Comparisons of the mean verification accuracy (\%) with several multiple metric learning methods and state-of-the-art methods on the KinFaceW-II dataset.

\begin{tabular}{|l|c|c|c|c|c|c|}
\hline Method & Feature & F-S & F-D & M-S & M-D & Mean \\
\hline \hline MCCA [135] & All & 74.0 & 72.1 & 74.8 & 75.3 & 74.1 \\
PMML [10] & All & 77.7 & 72.4 & 76.3 & 74.8 & 75.3 \\
MNRML [83] & All & 76.9 & 74.3 & 77.4 & 77.6 & 76.5 \\
DMML [150] & All & 78.5 & 76.5 & 78.5 & 79.5 & 78.3 \\
\hline \hline LM $^{3}$ L & All & 82.4 & 74.2 & 79.6 & 78.7 & 78.7 \\
L $^{3}{ }^{3}$ L & All & 82.4 & 78.2 & 78.8 & 80.4 & 80.0 \\
\hline \hline SILD (HOG) [114] & - & 79.6 & 71.6 & 73.2 & 69.6 & 73.5 \\
ULPGC [114] & - & 85.4 & 75.8 & 75.6 & 81.6 & 80.0 \\
BIU [114] & - & 87.5 & 80.8 & 79.8 & 75.6 & 80.9 \\
Polito [114] & - & 84.0 & 82.2 & 84.8 & 81.2 & 83.1 \\
LIRIS [114] & - & 89.4 & 83.6 & 86.2 & 85.0 & 86.1 \\
\hline
\end{tabular}

observe that $\mathrm{L}^{2} \mathrm{M}^{3} \mathrm{~L}$ achieves about $1.7 \%$ improvement over DMML and $1.3 \%$ over $\mathrm{LM}^{3} \mathrm{~L}$ in terms of the mean verification accuracy for kinship verification.

To be consistent with several multiple metric learning methods, MCCA [135], PMML [10], MNRML [83] and DMML [150], in our experiments, we followed the evaluation protocol and extracted the same features used in these methods. Therefore we didn't follow the evaluation protocol released in [114]. We also included the current state-of-the-art methods from [114] for comparison (see Table 5.10). From the Table 5.10, we see that $\mathrm{L}^{2} \mathrm{M}^{3} \mathrm{~L}$ outperforms the multiple metric learning methods, and methods from [114] show the better performance than $\mathrm{L}^{2} \mathrm{M}^{3} \mathrm{~L}$. The reason is that methods from [114] exploited more features and also used the high-dimensional features to boost the verification accuracy.

\subsubsection{Discussion and Parameter Analysis}

We examined several parameters that may affect the performance of the $\mathrm{LM}^{3} \mathrm{~L}$ and $\mathrm{L}^{2} \mathrm{M}^{3} \mathrm{~L}$ methods on the LFW dataset under category of image-restricted, label-free outside data. 


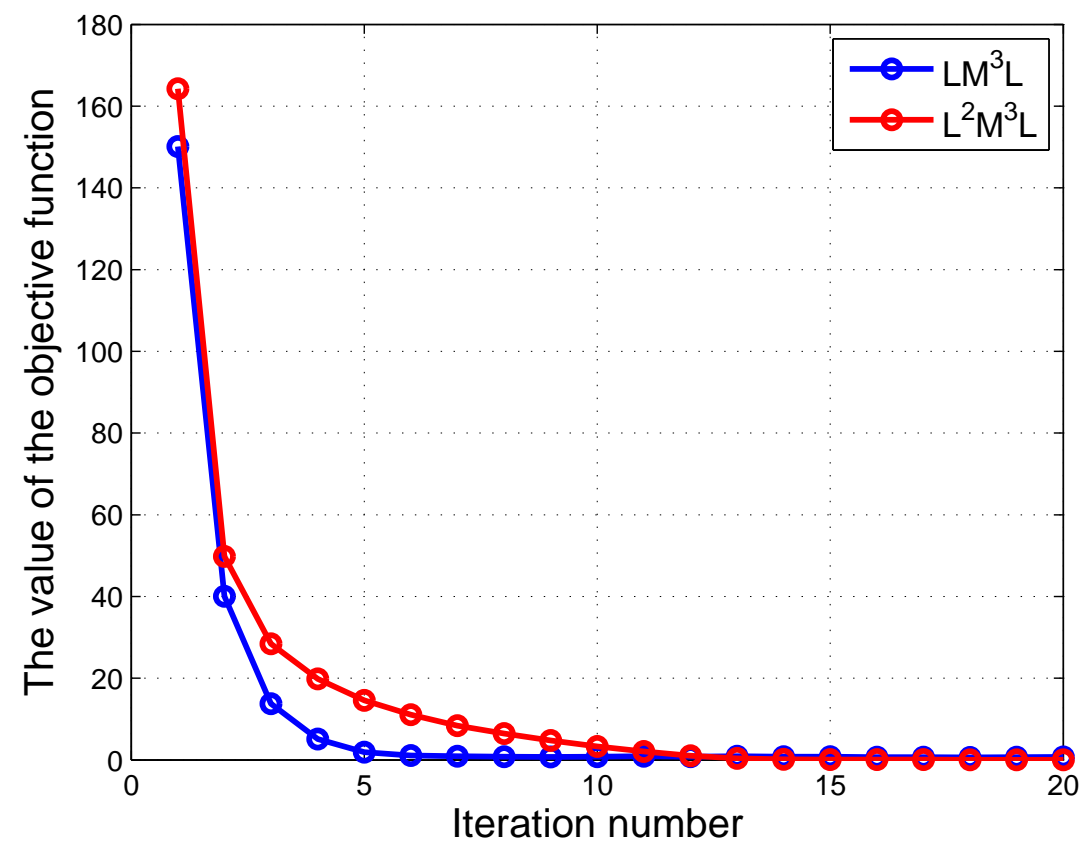

Figure 5.5: The value of the objective function of $\mathrm{LM}^{3} \mathrm{~L}$ and $\mathrm{L}^{2} \mathrm{M}^{3} \mathrm{~L}$ versus different number of iterations on the LFW dataset.

\subsubsection{Convergence Analysis}

We first evaluated the convergence of the $\mathrm{LM}^{3} \mathrm{~L}$ and $\mathrm{L}^{2} \mathrm{M}^{3} \mathrm{~L}$ methods with different number of iterations. Figure 5.5 shows the value of the objective function of the $\mathrm{LM}^{3} \mathrm{~L}$ and $\mathrm{L}^{2} \mathrm{M}^{3} \mathrm{~L}$ versus different number of iterations on the LFW dataset. We see that the convergence speed of our methods is acceptable. The $\mathrm{LM}^{3} \mathrm{~L}$ converges in $5 \sim 6$ iterations and the $\mathrm{L}^{2} \mathrm{M}^{3} \mathrm{~L}$ method begins to keep stable after the 10 iterations on the training set.

\subsubsection{Effect of Different Feature Dimensions}

Then we investigated the performance of the $\mathrm{LM}^{3} \mathrm{~L}$ and large-margin versus different feature dimensions. Figure 5.6 shows the mean verification accuracy of our multi-metric learning methods versus different feature dimensions on the LFW dataset. We notice that our methods can achieve stable performance when the feature dimension of various features 


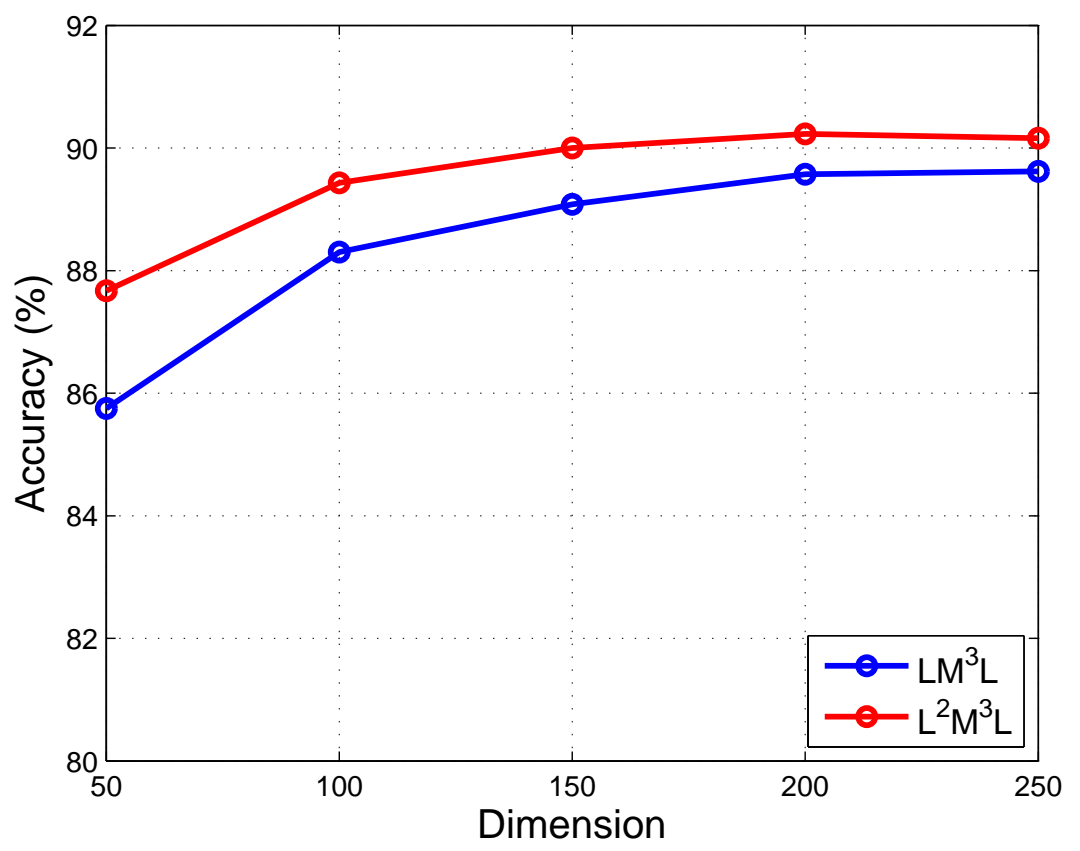

Figure 5.6: The mean verification accuracy of $\mathrm{LM}^{3} \mathrm{~L}$ and $\mathrm{L}^{2} \mathrm{M}^{3} \mathrm{~L}$ versus different feature dimensions on the LFW dataset.

reaches 200. This is the reason that we select 200 dimension for each feature via WPCA in our experiments on this dataset.

\subsubsection{Effect of Different Number of Local Metrics}

Lastly, we evaluated how the various number of local metrics (i.e., $Q_{k}$ ) affects the LML and $\mathrm{L}^{2} \mathrm{M}^{3} \mathrm{~L}$ methods. For the LML method, we chose SSIFT feature for this evaluation due to its good performance. Figure 5.7 lists the mean verification accuracy versus various $Q_{k}$ on the LFW dataset. We see that increasing $Q_{k}$ improves the accuracy of LML and $\mathrm{L}^{2} \mathrm{M}^{3} \mathrm{~L}$, but the performance of our local metric learning based methods remains stable or may even degrade if a too large number of local metrics is adopted. The reason may be that learning more local metrics requires a sufficient number of training samples in model training. In the experiments, we adopted three local metrics because it not only obtains acceptable results but also reduces the computational time. 


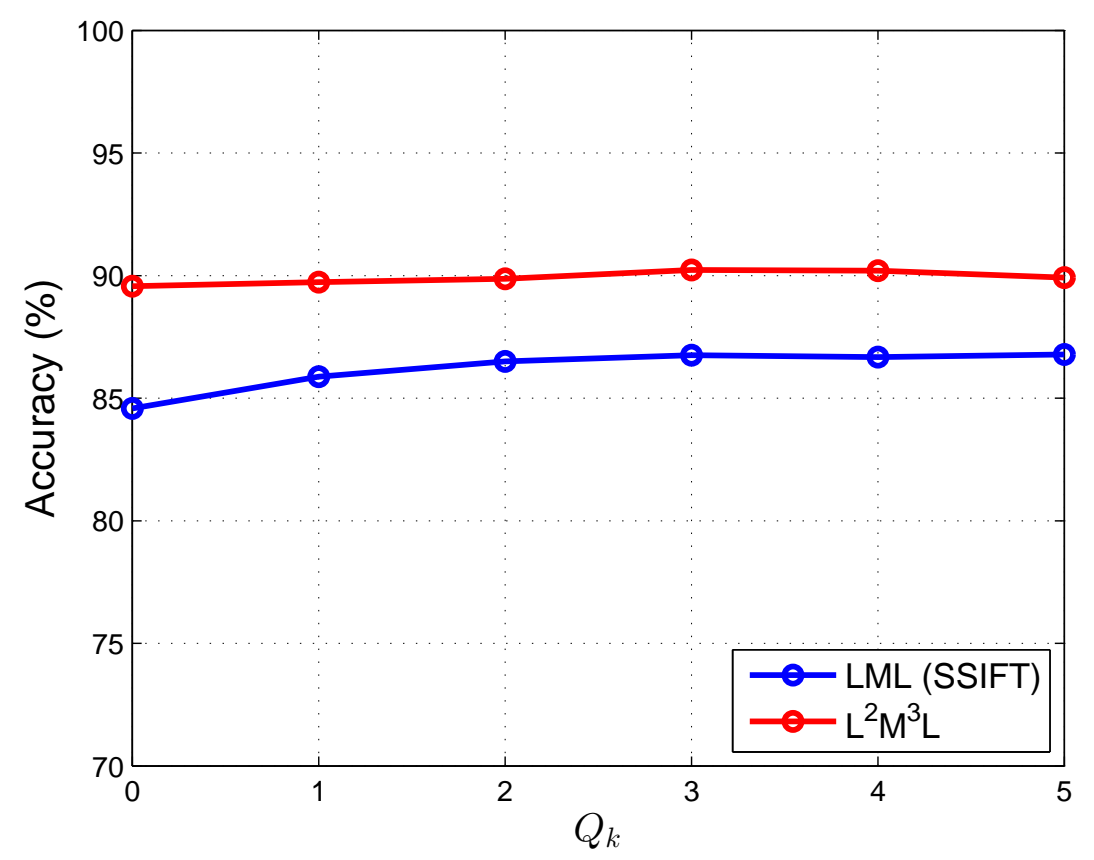

Figure 5.7: The mean verification accuracy of $\mathrm{LM}^{3} \mathrm{~L}$ and $\mathrm{L}^{2} \mathrm{M}^{3} \mathrm{~L}$ versus various $Q_{k}$ (i.e., number of local metrics) on the LFW dataset.

\subsubsection{Discussion on why minimizing $\left|d_{\mathbf{M}_{k}}\left(\mathbf{x}_{i}^{k}, \mathbf{x}_{j}^{k}\right)-d_{\mathbf{M}_{\ell}}\left(\mathbf{x}_{i}^{\ell}, \mathbf{x}_{j}^{\ell}\right)\right|$ will increase the correlation of $\mathrm{x}_{i}^{k}$ and $\mathrm{x}_{i}^{\ell}$}

Minimizing $\left|d_{\mathbf{M}_{k}}\left(\mathbf{x}_{i}^{k}, \mathbf{x}_{j}^{k}\right)-d_{\mathbf{M}_{\ell}}\left(\mathbf{x}_{i}^{\ell}, \mathbf{x}_{j}^{\ell}\right)\right|$ is not completely equivalent to increasing the correlation of $\mathbf{x}_{i}^{k}$ and $\mathbf{x}_{i}^{\ell}$, because $\mathbf{x}_{i}^{k}$ and $\mathbf{x}_{i}^{\ell}$ usually have different dimensions in both the original space and the transformed space such that it is infeasible to compute them directly. To show a simple analysis, we consider a special case that $\mathbf{x}_{i}^{k}$ and $\mathbf{x}_{i}^{\ell}$ are with same feature dimension in the transformed space. Under this special case, increasing the correlation of $\mathbf{x}_{i}^{k}$ and $\mathbf{x}_{i}^{\ell}$ means minimizing the following distance difference in the transformed space:

$$
d\left(\mathbf{L}_{k} \mathbf{x}_{i}^{k}, \mathbf{L}_{\ell} \mathbf{x}_{i}^{\ell}\right)=\left\|\mathbf{L}_{k} \mathbf{x}_{i}^{k}-\mathbf{L}_{\ell} \mathbf{x}_{i}^{\ell}\right\|_{2}
$$

where $\mathbf{L}_{k} \in \mathbb{R}^{s \times d_{k}}$ and $\mathbf{L}_{\ell} \in \mathbb{R}^{s \times d_{\ell}}, s<\min \left\{d_{k}, d_{\ell}\right\}$, are two linear transformations which map $\mathbf{x}_{i}^{k}$ and $\mathbf{x}_{i}^{\ell}$ from the original space to the transformed space as $\mathbf{L}_{k} \mathbf{x}_{i}^{k}$ and $\mathbf{L}_{\ell} \mathbf{x}_{i}^{\ell}$. For a 
sample pair $\mathbf{x}_{i}$ and $\mathbf{x}_{j}$, we have:

$$
\begin{aligned}
& d\left(\mathbf{L}_{k} \mathbf{x}_{i}^{k}, \mathbf{L}_{\ell} \mathbf{x}_{i}^{\ell}\right)+d\left(\mathbf{L}_{k} \mathbf{x}_{j}^{k}, \mathbf{L}_{\ell} \mathbf{x}_{j}^{\ell}\right) \\
& =\left\|\mathbf{L}_{k} \mathbf{x}_{i}^{k}-\mathbf{L}_{\ell} \mathbf{x}_{i}^{\ell}\right\|_{2}+\left\|\mathbf{L}_{k} \mathbf{x}_{j}^{k}-\mathbf{L}_{\ell} \mathbf{x}_{j}^{\ell}\right\|_{2} \\
& \geq\left\|\left(\mathbf{L}_{k} \mathbf{x}_{i}^{k}-\mathbf{L}_{\ell} \mathbf{x}_{i}^{\ell}\right)-\left(\mathbf{L}_{k} \mathbf{x}_{j}^{k}-\mathbf{L}_{\ell} \mathbf{x}_{j}^{\ell}\right)\right\|_{2} \\
& =\left\|\mathbf{L}_{k}\left(\mathbf{x}_{i}^{k}-\mathbf{x}_{j}^{k}\right)-\mathbf{L}_{\ell}\left(\mathbf{x}_{i}^{\ell}-\mathbf{x}_{j}^{\ell}\right)\right\|_{2} \\
& \geq\left|\left\|\mathbf{L}_{k}\left(\mathbf{x}_{i}^{k}-\mathbf{x}_{j}^{k}\right)\right\|_{2}-\left\|\mathbf{L}_{\ell}\left(\mathbf{x}_{i}^{\ell}-\mathbf{x}_{j}^{\ell}\right)\right\|_{2}\right| \\
& =\left|d_{\mathbf{M}_{k}}\left(\mathbf{x}_{i}^{k}, \mathbf{x}_{j}^{k}\right)-d_{\mathbf{M}_{\ell}}\left(\mathbf{x}_{i}^{\ell}, \mathbf{x}_{j}^{\ell}\right)\right|,
\end{aligned}
$$

where $\mathbf{M}_{k}=\mathbf{L}_{k}{ }^{T} \mathbf{L}_{k}$ and $d_{\mathbf{M}_{k}}\left(\mathbf{x}_{i}^{k}, \mathbf{x}_{j}^{k}\right)=\left\|\mathbf{L}_{k}\left(\mathbf{x}_{i}^{k}-\mathbf{x}_{j}^{k}\right)\right\|_{2}$. In (5.37), we use the reverse triangle inequality of vector norm axioms, that is $\|\mathbf{u}-\mathbf{v}\|_{2} \geq\left|\|\mathbf{u}\|_{2}-\|\mathbf{v}\|_{2}\right|$ for vectors $\mathbf{u}$ and $\mathbf{v}$. This simple analysis shows that minimizing $\left|d_{\mathbf{M}_{k}}\left(\mathbf{x}_{i}^{k}, \mathbf{x}_{j}^{k}\right)-d_{\mathbf{M}_{\ell}}\left(\mathbf{x}_{i}^{\ell}, \mathbf{x}_{j}^{\ell}\right)\right|$ can increase the correlation of $\mathbf{x}_{i}^{k}$ and $\mathbf{x}_{i}^{\ell}$ in the transformed space.

In addition, we also show this from an experimental point of view. We employ cosine similarity to measure the correlation of $\mathbf{x}_{i}^{k}$ and $\mathbf{x}_{i}^{\ell}$ when $\mathrm{x}_{i}^{k}$ and $\mathbf{x}_{i}^{\ell}$ have the same dimension, which is computed as follows:

$$
\cos \left(\mathbf{x}_{i}^{k}, \mathbf{x}_{i}^{\ell}\right)=\frac{\mathbf{x}_{i}^{k^{T}} \mathbf{x}_{i}^{\ell}}{\left\|\mathbf{x}_{i}^{k}\right\|_{2}\left\|\mathbf{x}_{i}^{\ell}\right\|_{2}} .
$$

Figure 5.8 shows the distribution of cosine similarity of $\mathrm{x}_{i}^{k}$ (i.e., LBP) and $\mathrm{x}_{i}^{\ell}$ (i.e., DSIFT) before (Blue: Original space) and after (Red: Transformed space) our metric learning method on LFW dataset under category of image-restricted, label-free outside data. From this figure, we can see that minimizing $\left|d_{\mathbf{M}_{k}}\left(\mathbf{x}_{i}^{k}, \mathbf{x}_{j}^{k}\right)-d_{\mathbf{M}_{\ell}}\left(\mathbf{x}_{i}^{\ell}, \mathbf{x}_{j}^{\ell}\right)\right|$ significantly increases the cosine similarity of $\mathbf{x}_{i}^{k}$ and $\mathbf{x}_{i}^{\ell}(k \neq \ell)$ in the transformed space by our method on both training data and test data. These results partially show that minimizing $\left|d_{\mathbf{M}_{k}}\left(\mathbf{x}_{i}^{k}, \mathbf{x}_{j}^{k}\right)-d_{\mathbf{M}_{\ell}}\left(\mathbf{x}_{i}^{\ell}, \mathbf{x}_{j}^{\ell}\right)\right|$ can increase the correlation of $\mathbf{x}_{i}^{k}$ and $\mathbf{x}_{i}^{\ell}$ in the transformed space. 


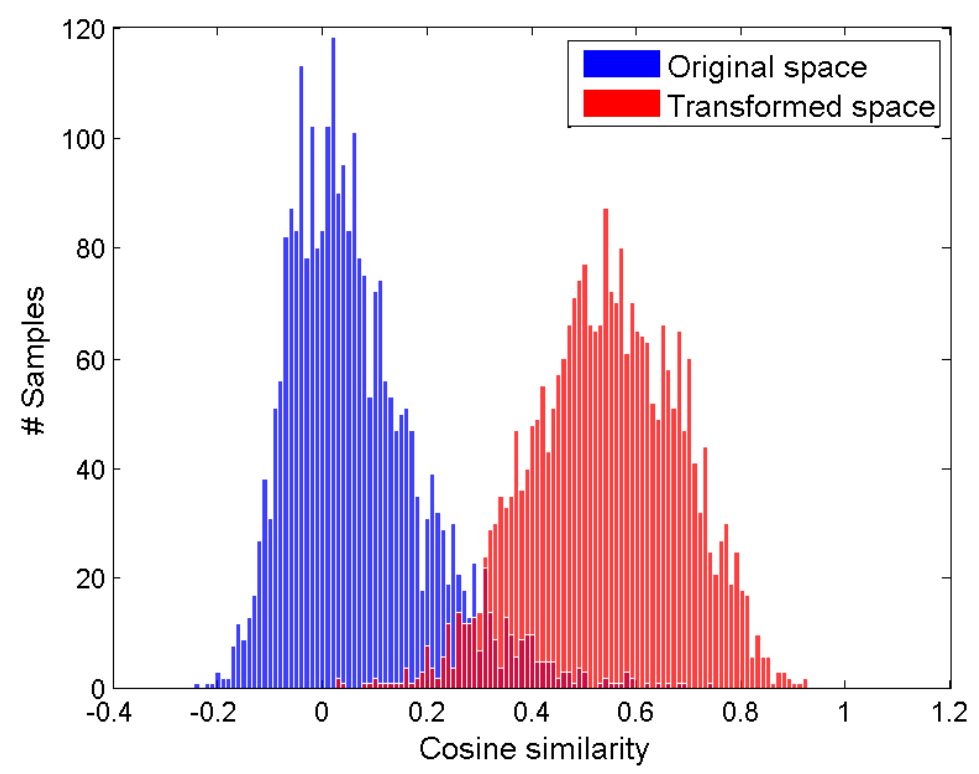

(a) Training data

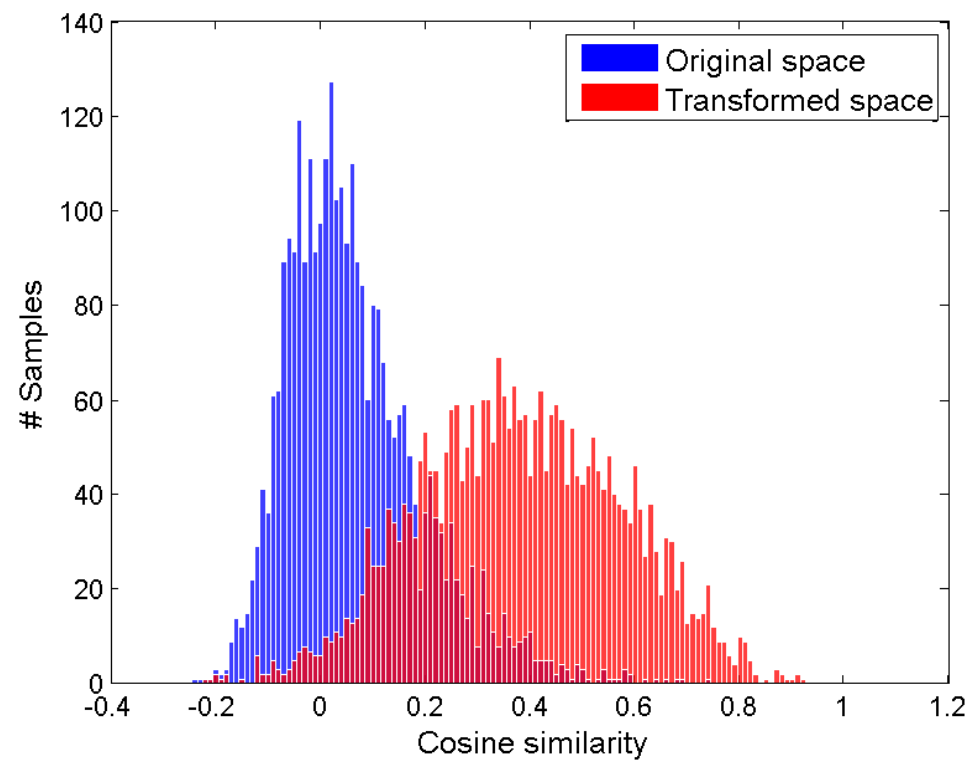

(b) Test data

Figure 5.8: Distribution of cosine similarity of $\mathbf{x}_{i}^{k}$ (i.e., LBP) and $\mathbf{x}_{i}^{\ell}$ (i.e., DSIFT) before (Blue: Original space) and after (Red: Transformed space) our method on LFW dataset under category of image-restricted, label-free outside data. 


\subsection{Conclusion}

In this chapter, we have introduced a large-margin multi-metric learning $\left(\mathrm{LM}^{3} \mathrm{~L}\right)$ method for face and kinship verification under unconstrained conditions. The $\mathrm{LM}^{3} \mathrm{~L}$ jointly learns multiple distance metrics under which more discriminative and complementary information can be exploited. Moreover, to better exploit the local structures of face images, we have proposed a local metric learning (LML) and a local large-margin multi-metric learning $\left(\mathrm{L}^{2} \mathrm{M}^{3} \mathrm{~L}\right)$ methods to learn a set of local metrics. Experimental results on three datasets show that our method can achieve competitive results compared with the state-of-the-art methods. The main work presented in this chapter has been published in $[130,131]$.

In the next chapter, we will conclude this thesis and list several directions that we can attempt in the future. 


\section{Chapter 6}

\section{Conclusion and Future Work}

\subsection{Conclusion}

Distance metric learning is a fundamental problem in machine learning, computer vision and pattern recognition. Numerous metric learning algorithms have been introduced over the past decade, and some of them have been successfully applied to various applications of visual analysis. However, there are a number of issues that remain to be improved for better efficiency. In this thesis, we contribute to the family of metric learning. At the very beginning, we review the existing works in metric learning, then we present several deep metric leaning methods and multi-view metric learning methods, and apply them to various visual recognition applications to demonstrate their effectiveness. The main contributions of the thesis are summarized below.

In Chapter 3, we have proposed a discriminative deep metric learning (DDML) method to explicitly address both the nonlinearity and scalability problems by leveraging a deep neural network architecture. The proposed DDML trains a neural network which learns a set of hierarchical nonlinear transformations to project sample pairs into the same feature subspace, under which the distance of each positive pair is less than a small threshold and that of each negative pair is higher than a large threshold respectively, such that more discriminative information can be exploited in the neural network. We have applied DDML to 
both face verification and kinship verification under the unconstrained conditions. Extensive experiments on four challenging benchmark datasets demonstrate the efficacy of the proposed approach.

In Chapter 4, we have introduced a deep transfer metric learning (DTML) method to address the nonlinearity issue and the fact that the training and test samples are usually from different distributions in many real-world visual applications. The proposed DTML learns a set of hierarchical nonlinear transformations for cross-domain visual recognition by transferring discriminative knowledge from the labeled source domain to the unlabeled target domain. Specifically, DTML learns a deep metric network by maximizing the interclass variations and minimizing the intra-class variations, and minimizing the distribution divergence between the source domain and the target domain at the top layer of the network. To better exploit the discriminative information from the source domain, we have further developed a deeply supervised transfer metric learning (DSTML) method by including an additional objective on DTML, where the output of both the hidden layers and the top layer are optimized jointly. To preserve the local manifold of input data points, we have proposed DTML with autoencoder regularization (DTML-AE) and DSTML with autoencoder regularization (DSTML-AE) methods. Experimental results on face verification, person re-identification, and handwritten digit recognition validate the effectiveness of the proposed DTML and its variants.

Lastly, Chapter 5 has proposed a large-margin multi-metric learning $\left(\mathrm{LM}^{3} \mathrm{~L}\right)$ method to explore multiple feature representations of data. Unlike the methods of learning a distance metric on the concatenated feature vectors, we collaboratively learn multiple distance metrics from multiple feature representations of data, where one distance metric is learned for each feature and the correlations of different feature representations of each sample are maximized, and under the learned metric spaces the distance of each positive pair is 
less than a small threshold and that of each negative pair is more than a large threshold, respectively. To better utilize the local manifold structures of data points, we have further proposed two local distance metric learning approaches, i.e., local metric learning (LML) and local large-margin multi-metric learning $\left(\mathrm{L}^{2} \mathrm{M}^{3} \mathrm{~L}\right)$. The proposed methods have been evaluated by both face verification and kinship verification tasks. Experimental results on a number of datasets show that the proposed multi-view metric leaning methods can obtain competitive results compared with state-of-the-art methods.

\subsection{Future Work}

While the works presented in this thesis have achieved promising performance for several visual recognition applications, they can likely be further improved or exploited in various ways. In this subsection, we present some feasible ideas that can be attempted in the future:

- The proposed methods can be applied to image retrieval. Similarity search is a basic issue in content-based image retrieval (CBIR). Due to the semantic gap between the low-level visual features and high-level human perception, the hand-crafted similarity or distance functions may not be powerful to tackle the CBIR, and metric learning methods are utilized to learn distance metrics from training data in an supervised manner. We would adopt the proposed deep metric learning approaches to learn several flexible nonlinear similarity functions for image retrieval task.

- In this thesis, we formulate deep metric learning methods by using the feed-forward artificial neural network. We can extend our methods to other deep learning architectures, for example, deep belief networks (DBN) [49], and convolutional neural networks (CNN) [5,45]. The CNN has been successfully introduced in various visual tasks such as image classification [45] and face verification [24], because it can learn 
high-level hierarchical feature representations directly from raw data. By integrating the deep metric learning and $\mathrm{CNN}$, the similarity function and feature representation can be learned simultaneously, which should improve the performance of the previous deep metric learning for visual recognition.

- We plan to further investigate the implementation details in deep metric learning algorithms, including nonlinear activation function, pre-training technique, data augmentation, the learning rate, and reducing overfitting in optimization.

- We may consider other formulations of multi-view metric learning to exploit multiple features and further integrate them with state-of-the-art deep learning algorithms to study multi-view deep metric learning.

- Most existing deep metric learning methods are supervised. In many real-world applications, it is easier to collect an extensive unlabeled data rather than labeled data for practical applications. Hence, how to develop more effective unsupervised or semi-supervised deep metric learning is an important future direction.

- Most existing deep metric learning methods are developed for a single specific task, which means that a large amount of labeled data for this task are usually required to exploit the supervision information. In some real-world applications, it is difficult to collect extensive labeled data for a specific task. Therefore, it is desirable to conduct multi-task deep metric learning which can leverage labeled samples from multiple different yet related tasks so that it is much easier to obtain more labeled samples for deep metric learning, which is also an interesting future research direction. 


\section{Author's Publications}

[1] Junlin Hu, Jiwen Lu, Yap-Peng Tan, Jie Zhou, "Deep transfer metric learning", IEEE Transactions on Image Processing, 25(12): 5576-5588, 2016.

[2] Junlin Hu, Jiwen Lu, Yap-Peng Tan, "Deep metric learning for visual tracking”, IEEE Transactions on Circuits and Systems for Video Technology, 26(11): 2056-2068, 2016.

[3] Junlin Hu, Jiwen Lu, Yap-Peng Tan, Junsong Yuan, Jie Zhou, "Local large-margin multimetric learning for face and kinship verification", IEEE Transactions on Circuits and Systems for Video Technology, 2017, DOI: 10.1109/TCSVT.2017.2691801.

[4] Jiwen Lu, Junlin Hu, Yap-Peng Tan, "Discriminative deep metric learning for face and kinship verification”, IEEE Transactions on Image Processing, 26(9): 4269-4282, 2017.

[5] Junlin Hu, Jiwen Lu, Yap-Peng Tan, "Sharable and individual multi-view metric learning", IEEE Transactions on Pattern Analysis and Machine Intelligence, 2017, DOI: 10.1109/TPAMI.2017.2749576.

[6] Junlin Hu, and Yap-Peng Tan, "Nonlinear dictionary learning with application to image classification”, Pattern Recognition, 75: 282-291, 2018.

[7] Junlin Hu, Jiwen Lu, Yap-Peng Tan, "Deep transfer metric learning", IEEE Conference on Computer Vision and Pattern Recognition (CVPR), pp. 325-333, 2015.

[8] Junlin Hu, Jiwen Lu, Yap-Peng Tan, "Discriminative deep metric learning for face verification in the wild", IEEE Conference on Computer Vision and Pattern Recognition (CVPR), pp. 1875-1882, 2014.

[9] Junlin Hu, Jiwen Lu, Junsong Yuan, Yap-Peng Tan, "Large margin multi-metric learning for face and kinship verification in the wild", Asian Conference on Computer Vision (ACCV), pp. 252-267, 2014.

[10] Junlin Hu, Jiwen Lu, Junsong Yuan, Yap-Peng Tan, "Collaborative multi-view metric learning for visual classification", IEEE International Conference on Multimedia and Expo (ICME), 2016.

[11] Junlin Hu, Jiwen Lu, Yap-Peng Tan, "Fine-grained face verification: Dataset and baseline results", International Conference on Biometrics (ICB), 2015.

[12] Junlin Hu, Jiwen Lu, Xiuzhuang Zhou, Yap-Peng Tan, "Discriminative transfer learning for single-sample face recognition", International Conference on Biometrics (ICB), 2015. 


\section{Bibliography}

[1] B. Kulis, "Metric learning: A survey," Foundations and Trends in Machine Learning, vol. 5, no. 4, pp. 287-364, 2013.

[2] E. P. Xing, A. Y. Ng, M. I. Jordan, and S. J. Russell, "Distance metric learning with application to clustering with side-information," in Advances in Neural Information Processing Systems, 2002, pp. 505-512.

[3] J. V. Davis, B. Kulis, P. Jain, S. Sra, and I. S. Dhillon, "Information-theoretic metric learning," in International Conference on Machine Learning, 2007, pp. 209-216.

[4] K. Q. Weinberger and L. K. Saul, "Distance metric learning for large margin nearest neighbor classification,” Journal of Machine Learning Research, vol. 10, pp. 207-244, 2009.

[5] Y. LeCun, L. Bottou, Y. Bengio, and P. Haffner, "Gradient-based learning applied to document recognition," Proceedings of the IEEE, vol. 86, no. 11, pp. 2278-2324, 1998.

[6] P. N. Belhumeur, J. P. Hespanha, and D. J. Kriegman, "Eigenfaces vs. fisherfaces: Recognition using class specific linear projection," IEEE Transactions on Pattern Analysis and Machine Intelligence, vol. 19, no. 7, pp. 711-720, 1997.

[7] M. Guillaumin, J. J. Verbeek, and C. Schmid, "Is that you? metric learning approaches for face identification," in IEEE International Conference on Computer Vision, 2009, pp. 498505.

[8] H. V. Nguyen and L. Bai, "Cosine similarity metric learning for face verification," in Asian Conference on Computer Vision, 2010, pp. 709-720.

[9] M. Köstinger, M. Hirzer, P. Wohlhart, P. M. Roth, and H. Bischof, "Large scale metric learning from equivalence constraints," in IEEE Conference on Computer Vision and Pattern Recognition, 2012, pp. 2288-2295.

[10] Z. Cui, W. Li, D. Xu, S. Shan, and X. Chen, "Fusing robust face region descriptors via multiple metric learning for face recognition in the wild," in IEEE Conference on Computer Vision and Pattern Recognition, 2013, pp. 3554-3561.

[11] J. Hu, J. Lu, and Y.-P. Tan, "Discriminative deep metric learning for face verification in the wild," in IEEE Conference on Computer Vision and Pattern Recognition, 2014, pp. 18751882 . 
[12] Z. Huang, R. Wang, S. Shan, and X. Chen, "Projection metric learning on grassmann manifold with application to video based face recognition," in IEEE Conference on Computer Vision and Pattern Recognition, 2015, pp. 140-149.

[13] D. Tran and A. Sorokin, "Human activity recognition with metric learning," in European Conference on Computer Vision, 2008, pp. 548-561.

[14] M. Norouzi, D. J. Fleet, and R. Salakhutdinov, "Hamming distance metric learning," in Advances in Neural Information Processing Systems, 2012, pp. 1070-1078.

[15] D.-Y. Yeung and H. Chang, "A kernel approach for semisupervised metric learning," IEEE Transactions on Neural Networks, vol. 18, no. 1, pp. 141-149, 2007.

[16] R. Wang, S. Shan, X. Chen, Q. Dai, and W. Gao, "Manifold-manifold distance and its application to face recognition with image sets," IEEE Transactions on Image Processing, vol. 21, no. 10 , pp. 4466-4479, 2012.

[17] Z. Huang, R. Wang, S. Shan, and X. Chen, "Learning euclidean-to-riemannian metric for point-to-set classification," in IEEE Conference on Computer Vision and Pattern Recognition, 2014, pp. 1677-1684.

[18] M. Kan, S. Shan, D. Xu, and X. Chen, "Side-information based linear discriminant analysis for face recognition." in British Machine Vision Conference, vol. 11, 2011, pp. 125-136.

[19] I. W. Tsang and J. T. Kwok, "Distance metric learning with kernels," in International Conference on Artificial Neural Networks, 2003, pp. 126-129.

[20] S. Chopra, R. Hadsell, and Y. LeCun, "Learning a similarity metric discriminatively, with application to face verification," in IEEE Conference on Computer Vision and Pattern Recognition, 2005, pp. 539-546.

[21] R. Hadsell, S. Chopra, and Y. LeCun, "Dimensionality reduction by learning an invariant mapping," in IEEE Conference on Computer Vision and Pattern Recognition, 2006, pp. $1735-1742$.

[22] X. Cai, C. Wang, B. Xiao, X. Chen, and J. Zhou, "Deep nonlinear metric learning with independent subspace analysis for face verification," in ACM International Conference on Multimedia, 2012, pp. 749-752.

[23] P. Wu, S. C. H. Hoi, H. Xia, P. Zhao, D. Wang, and C. Miao, "Online multimodal deep similarity learning with application to image retrieval," in ACM International Conference on Multimedia, 2013, pp. 153-162.

[24] Y. Taigman, M. Yang, M. Ranzato, and L. Wolf, "Deepface: Closing the gap to humanlevel performance in face verification," in IEEE Conference on Computer Vision and Pattern Recognition, 2014, pp. 1701-1708.

[25] D. Yi, Z. Lei, S. Liao, and S. Z. Li, "Deep metric learning for person re-identification," in International Conference on Pattern Recognition, 2014, pp. 34-39. 
[26] Y. Sun, Y. Chen, X. Wang, and X. Tang, "Deep learning face representation by joint identification-verification," in Advances in Neural Information Processing Systems, 2014, pp. 1988-1996.

[27] O. Batchelor and R. D. Green, "Object recognition by stochastic metric learning," in International Conference on Simulated Evolution And Learning, 2014, pp. 798-809.

[28] J. Wang, Y. Song, T. Leung, C. Rosenberg, J. Wang, J. Philbin, B. Chen, and Y. Wu, "Learning fine-grained image similarity with deep ranking," in IEEE Conference on Computer Vision and Pattern Recognition, 2014, pp. 1386-1393.

[29] J. Hu, J. Lu, and Y.-P. Tan, "Deep transfer metric learning," in IEEE Conference on Computer Vision and Pattern Recognition, 2015, pp. 325-333.

[30] F. Schroff, D. Kalenichenko, and J. Philbin, "Facenet: A unified embedding for face recognition and clustering," in IEEE Conference on Computer Vision and Pattern Recognition, 2015, pp. 815-823.

[31] J. Lu, G. Wang, W. Deng, P. Moulin, and J. Zhou, "Multi-manifold deep metric learning for image set classification," in IEEE Conference on Computer Vision and Pattern Recognition, 2015, pp. 1137-1145.

[32] X. Han, T. Leung, Y. Jia, R. Sukthankar, and A. C. Berg, "Matchnet: Unifying feature and metric learning for patch-based matching," in IEEE Conference on Computer Vision and Pattern Recognition, 2015, pp. 3279-3286.

[33] E. Hoffer and N. Ailon, "Deep metric learning using triplet network," in Similarity-Based Pattern Recognition - Third International Workshop, SIMBAD 2015, 2015, pp. 84-92.

[34] S. Bell and K. Bala, "Learning visual similarity for product design with convolutional neural networks," ACM Transactions on Graphics, vol. 34, no. 4, p. 98, 2015.

[35] J. Hu, J. Lu, and Y.-P. Tan, "Deep metric learning for visual tracking," IEEE Transactions on Circuits and Systems for Video Technology, vol. 26, no. 11, pp. 2056-2068, 2016.

[36] H. O. Song, Y. Xiang, S. Jegelka, and S. Savarese, "Deep metric learning via lifted structured feature embedding," in IEEE Conference on Computer Vision and Pattern Recognition, 2016, pp. 4004-4012.

[37] Y. Cui, F. Zhou, Y. Lin, and S. Belongie, "Fine-grained categorization and dataset bootstrapping using deep metric learning with humans in the loop," in IEEE Conference on Computer Vision and Pattern Recognition, 2016, pp. 1153-1162.

[38] H. Shi, Y. Yang, X. Zhu, S. Liao, Z. Lei, W. Zheng, and S. Z. Li, "Embedding deep metric for person re-identification: A study against large variations," in European Conference on Computer Vision, 2016, pp. 732-748.

[39] K. Sohn, "Improved deep metric learning with multi-class n-pair loss objective," in Advances in Neural Information Processing Systems, 2016, pp. 1849-1857. 
[40] I. Lim, A. Gehre, and L. Kobbelt, "Identifying style of 3d shapes using deep metric learning," Computer Graphics Forum, vol. 35, no. 5, pp. 207-215, 2016.

[41] J. Hu, J. Lu, and Y.-P. Tan, "Sharable and individual multi-view metric learning," IEEE Transactions on Pattern Analysis and Machine Intelligence, 2017, DOI: 10.1109/TPAMI.2017.2749576.

[42] H. Lai, Y. Pan, Y. Liu, and S. Yan, "Simultaneous feature learning and hash coding with deep neural networks," in IEEE Conference on Computer Vision and Pattern Recognition, 2015, pp. 3270-3278.

[43] V. E. Liong, J. Lu, G. Wang, P. Moulin, and J. Zhou, "Deep hashing for compact binary codes learning," in IEEE Conference on Computer Vision and Pattern Recognition, 2015, pp. $2475-2483$.

[44] H. Li, Y. Li, and F. Porikli, "Deeptrack: Learning discriminative feature representations online for robust visual tracking," IEEE Transactions on Image Processing, vol. 25, no. 4, pp. 1834-1848, 2016.

[45] A. Krizhevsky, I. Sutskever, and G. E. Hinton, "Imagenet classification with deep convolutional neural networks," in Advances in Neural Information Processing Systems, 2012, pp. 1106-1114.

[46] C. Szegedy, A. Toshev, and D. Erhan, "Deep neural networks for object detection," in Advances in Neural Information Processing Systems, 2013, pp. 2553-2561.

[47] Q. V. Le, W. Y. Zou, S. Y. Yeung, and A. Y. Ng, "Learning hierarchical invariant spatiotemporal features for action recognition with independent subspace analysis," in IEEE Conference on Computer Vision and Pattern Recognition, 2011, pp. 3361-3368.

[48] G. B. Huang, H. Lee, and E. G. Learned-Miller, "Learning hierarchical representations for face verification with convolutional deep belief networks," in IEEE Conference on Computer Vision and Pattern Recognition, 2012, pp. 2518-2525.

[49] G. E. Hinton, S. Osindero, and Y. W. Teh, "A fast learning algorithm for deep belief nets," Neural Computation, vol. 18, no. 7, pp. 1527-1554, 2006.

[50] C. Lee, S. Xie, P. Gallagher, Z. Zhang, and Z. Tu, "Deeply-supervised nets," in International Conference on Artificial Intelligence and Statistics, 2015, pp. 562-570.

[51] S. J. Pan and Q. Yang, "A survey on transfer learning," IEEE Transactions on Knowledge and Data Engineering, vol. 22, no. 10, pp. 1345-1359, 2010.

[52] M. Long, J. Wang, G. Ding, S. J. Pan, and P. S. Yu, "Adaptation regularization: A general framework for transfer learning," IEEE Transactions on Knowledge and Data Engineering, vol. 26, no. 5, pp. 1076-1089, 2014.

[53] W. Li, L. Duan, D. Xu, and I. W. Tsang, "Learning with augmented features for supervised and semi-supervised heterogeneous domain adaptation," IEEE Transactions on Pattern Analysis and Machine Intelligence, vol. 36, no. 6, pp. 1134-1148, 2014. 
[54] L. Zhang and D. Zhang, "Robust visual knowledge transfer via extreme learning machinebased domain adaptation," IEEE Transactions on Image Processing, vol. 25, no. 10, pp. 4959-4973, 2016.

[55] W. Dai, Q. Yang, G. Xue, and Y. Yu, "Boosting for transfer learning," in International Conference on Machine Learning, 2007, pp. 193-200.

[56] R. K. Ando and T. Zhang, "A framework for learning predictive structures from multiple tasks and unlabeled data," Journal of Machine Learning Research, vol. 6, pp. 1817-1853, 2005.

[57] L. Duan, I. W. Tsang, D. Xu, and S. J. Maybank, "Domain transfer SVM for video concep$\mathrm{t}$ detection," in IEEE Conference on Computer Vision and Pattern Recognition, 2009, pp. $1375-1381$.

[58] L. Duan, I. W. Tsang, and D. Xu, "Domain transfer multiple kernel learning," IEEE Transactions on Pattern Analysis and Machine Intelligence, vol. 34, no. 3, pp. 465-479, 2012.

[59] S. J. Pan, J. T. Kwok, and Q. Yang, "Transfer learning via dimensionality reduction," in AAAI Conference on Artificial Intelligence, 2008, pp. 677-682.

[60] S. Si, D. Tao, and B. Geng, "Bregman divergence-based regularization for transfer subspace learning," IEEE Transactions on Knowledge and Data Engineering, vol. 22, no. 7, pp. 929$942,2010$.

[61] C. Xu, D. Tao, and C. Xu, "Multi-view intact space learning," IEEE Transactions on Pattern Analysis and Machine Intelligence, vol. 37, no. 12, pp. 2531-2544, 2015.

[62] Z. Zha, T. Mei, M. Wang, Z. Wang, and X. Hua, "Robust distance metric learning with auxiliary knowledge," in International Joint Conference on Artificial Intelligence, 2009, pp. $1327-1332$.

[63] Y. Zhang and D.-Y. Yeung, "Transfer metric learning by learning task relationships," in ACM SIGKDD International Conference on Knowledge Discovery and Data Mining, 2010, pp. 1199-1208.

[64] _ _ "Transfer metric learning with semi-supervised extension," ACM Transactions on Intelligent Systems and Technology, vol. 3, no. 3, p. 54, 2012.

[65] W. Li, R. Zhao, and X. Wang, "Human reidentification with transferred metric learning," in Asian Conference on Computer Vision, 2012, pp. 31-44.

[66] Y. Luo, T. Liu, D. Tao, and C. Xu, "Decomposition-based transfer distance metric learning for image classification," IEEE Transactions on Image Processing, vol. 23, no. 9, pp. 37893801, 2014.

[67] Z. Al-Halah, L. Rybok, and R. Stiefelhagen, "Transfer metric learning for action similarity using high-level semantics," Pattern Recognition Letters, vol. 72, pp. 82-90, 2016. 
[68] Z. Ding and Y. Fu, "Robust transfer metric learning for image classification," IEEE Transactions on Image Processing, vol. 26, no. 2, pp. 660-670, 2017.

[69] G. B. Huang, M. Ramesh, T. Berg, and E. Learned-Miller, "Labeled faces in the wild: A database for studying face recognition in unconstrained environments," University of Massachusetts, Amherst, Tech. Rep. 07-49, 2007.

[70] L. Wolf, T. Hassner, and Y. Taigman, "Descriptor based methods in the wild," in Real-Life Images workshop at the European Conference on Computer Vision, 2008, pp. 1-14.

[71] _ _ "Similarity scores based on background samples," in Asian Conference on Computer Vision, 2009, pp. 88-97.

[72] D. Yi, Z. Lei, and S. Z. Li, “Towards pose robust face recognition,” in IEEE Conference on Computer Vision and Pattern Recognition, 2013, pp. 3539-3545.

[73] K. Simonyan, O. M. Parkhi, A. Vedaldi, and A. Zisserman, "Fisher vector faces in the wild," in British Machine Vision Conference, 2013, pp. 1-12.

[74] L. Wolf, T. Hassner, and I. Maoz, "Face recognition in unconstrained videos with matched background similarity," in IEEE Conference on Computer Vision and Pattern Recognition, 2011, pp. 529-534.

[75] L. Wolf and N. Levy, "The svm-minus similarity score for video face recognition," in IEEE Conference on Computer Vision and Pattern Recognition, 2013, pp. 3523-3530.

[76] Z. Huang, R. Wang, S. Shan, and X. Chen, "Face recognition on large-scale video in the wild with hybrid euclidean-and-riemannian metric learning," Pattern Recognition, vol. 48, no. 10, pp. 3113-3124, 2015.

[77] X. Jiang and J. Lai, "Sparse and dense hybrid representation via dictionary decomposition for face recognition," IEEE Transactions on Pattern Analysis and Machine Intelligence, vol. 37, no. 5, pp. 1067-1079, 2015.

[78] D. G. Lowe, "Distinctive image features from scale-invariant keypoints," International Journal of Computer Vision, vol. 60, no. 2, pp. 91-110, 2004.

[79] T. Ahonen, A. Hadid, and M. Pietikäinen, "Face description with local binary patterns: Application to face recognition," IEEE Transactions on Pattern Analysis and Machine Intelligence, vol. 28, no. 12, pp. 2037-2041, 2006.

[80] Z. Lei, M. Pietikäinen, and S. Z. Li, "Learning discriminant face descriptor," IEEE Transactions on Pattern Analysis and Machine Intelligence, vol. 36, no. 2, pp. 289-302, 2014.

[81] R. Fang, K. Tang, N. Snavely, and T. Chen, "Towards computational models of kinship verification," in International Conference on Image Processing, 2010, pp. 1577-1580.

[82] S. Xia, M. Shao, J. Luo, and Y. Fu, "Understanding kin relationships in a photo," IEEE Transactions on Multimedia, vol. 14, no. 4, pp. 1046-1056, 2012. 
[83] J. Lu, J. Hu, X. Zhou, Y. Shang, Y.-P. Tan, and G. Wang, "Neighborhood repulsed metric learning for kinship verification," in IEEE Conference on Computer Vision and Pattern Recognition, 2012, pp. 2594-2601.

[84] J. P. Robinson, M. Shao, Y. Wu, and Y. Fu, "Families in the wild (FIW): large-scale kinship image database and benchmarks," in ACM International Conference on Multimedia, 2016, pp. 242-246.

[85] X. Zhou, J. Hu, J. Lu, Y. Shang, and Y. Guan, "Kinship verification from facial images under uncontrolled conditions," in ACM International Conference on Multimedia, 2011, pp. 953956.

[86] X. Zhou, J. Lu, J. Hu, and Y. Shang, "Gabor-based gradient orientation pyramid for kinship verification under uncontrolled environments," in ACM International Conference on Multimedia, 2012, pp. 725-728.

[87] G. Somanath and C. Kambhamettu, "Can faces verify blood-relations?" in IEEE International Conference on Biometrics: Theory, Applications and Systems, 2012, pp. 105-112.

[88] H. Dibeklioglu, A. A. Salah, and T. Gevers, "Like father, like son: Facial expression dynamics for kinship verification," in IEEE International Conference on Computer Vision, 2013, pp. 1497-1504.

[89] Y. Guo, H. Dibeklioglu, and L. van der Maaten, "Graph-based kinship recognition," in International Conference on Pattern Recognition, 2014.

[90] G. Guo and X. Wang, "Kinship measurement on salient facial features," IEEE Transactions on Instrumentation and Measurement, vol. 61, no. 8, pp. 2322-2325, 2012.

[91] N. Kohli, R. Singh, and M. Vatsa, "Self-similarity representation of weber faces for kinship classification," in IEEE International Conference on Biometrics: Theory, Applications and Systems, 2012, pp. 245-250.

[92] W.-S. Zheng, S. Gong, and T. Xiang, "Reidentification by relative distance comparison," IEEE Transactions on Pattern Analysis and Machine Intelligence, vol. 35, no. 3, pp. 653668, 2013.

[93] A. J. Ma, P. C. Yuen, and J. Li, "Domain transfer support vector ranking for person reidentification without target camera label information," in IEEE International Conference on Computer Vision, 2013, pp. 3567-3574.

[94] F. Xiong, M. Gou, O. I. Camps, and M. Sznaier, "Person re-identification using kernel-based metric learning methods," in European Conference on Computer Vision, 2014, pp. 1-16.

[95] W.-S. Zheng, S. Gong, and T. Xiang, "Towards open-world person re-identification by oneshot group-based verification," IEEE Transactions on Pattern Analysis and Machine Intelligence, vol. 38, no. 3, pp. 591-606, 2016.

[96] J. Lu, J. Hu, and Y.-P. Tan, "Discriminative deep metric learning for face and kinship verification," IEEE Transactions on Image Processing, vol. 26, no. 9, pp. 4269-4282, 2017. 
[97] A. Mignon and F. Jurie, "PCCA: A new approach for distance learning from sparse pairwise constraints," in IEEE Conference on Computer Vision and Pattern Recognition, 2012, pp. 2666-2672.

[98] P. Vincent, H. Larochelle, Y. Bengio, and P.-A. Manzagol, "Extracting and composing robust features with denoising autoencoders," in International Conference on Machine Learning, 2008, pp. 1096-1103.

[99] X. Glorot and Y. Bengio, "Understanding the difficulty of training deep feedforward neural networks," in International Conference on Artificial Intelligence and Statistics, 2010, pp. 249-256.

[100] D. Chen, X. Cao, F. Wen, and J. Sun, "Blessing of dimensionality: High-dimensional feature and its efficient compression for face verification," in IEEE Conference on Computer Vision and Pattern Recognition, 2013, pp. 3025-3032.

[101] N. Dalal and B. Triggs, "Histograms of oriented gradients for human detection," in IEEE Conference on Computer Vision and Pattern Recognition, 2005, pp. 886-893.

[102] M. Kan, D. Xu, S. Shan, W. Li, and X. Chen, "Learning prototype hyperplanes for face verification in the wild," IEEE Transactions on Image Processing, vol. 22, no. 8, pp. 33103316, 2013.

[103] Y. Ying and P. Li, "Distance metric learning with eigenvalue optimization," Journal of Machine Learning Research, vol. 13, pp. 1-26, 2012.

[104] Q. Cao, Y. Ying, and P. Li, "Similarity metric learning for face recognition," in IEEE International Conference on Computer Vision, 2013, pp. 2408-2415.

[105] O. Barkan, J. Weill, L. Wolf, and H. Aronowitz, "Fast high dimensional vector multiplication face recognition," in IEEE International Conference on Computer Vision, 2013, pp. 1960 1967.

[106] S. Prince, P. Li, Y. Fu, U. Mohammed, and J. H. Elder, "Probabilistic models for inference about identity," IEEE Transactions on Pattern Analysis and Machine Intelligence, vol. 34, no. 1, pp. 144-157, 2012.

[107] D. Chen, X. Cao, L. Wang, F. Wen, and J. Sun, "Bayesian face revisited: A joint formulation," in European Conference on Computer Vision, 2012, pp. 566-579.

[108] Y. Sun, X. Wang, and X. Tang, "Hybrid deep learning for face verification," in IEEE International Conference on Computer Vision, 2013, pp. 1489-1496.

[109] O. M. Parkhi, A. Vedaldi, and A. Zisserman, "Deep face recognition," in British Machine Vision Conference, 2015, pp. 41.1-41.12.

[110] H. Li, G. Hua, Z. Lin, J. Brandt, and J. Yang, "Probabilistic elastic matching for pose variant face verification," in IEEE Conference on Computer Vision and Pattern Recognition, 2013, pp. 3499-3506. 
[111] H. M. Vazquez, Y. Martínez-Díaz, and Z. Chai, "Volume structured ordinal features with background similarity measure for video face recognition," in International Conference on Biometrics, 2013, pp. 1-6.

[112] Y. Sun, X. Wang, and X. Tang, "Deeply learned face representations are sparse, selective, and robust," in IEEE Conference on Computer Vision and Pattern Recognition, 2015, pp. 2892-2900.

[113] T. Ahonen, E. Rahtu, V. Ojansivu, and J. Heikkilä, "Recognition of blurred faces using local phase quantization," in International Conference on Pattern Recognition, 2008, pp. 1-4.

[114] J. Lu, J. Hu, V. E. Liong, X. Zhou, A. Bottino, I. U. Islam, T. F. Vieira, X. Qin, X. Tan, S. Chen, S. Mahpod, Y. Keller, L. Zheng, K. Idrissi, C. Garcia, S. Duffner, A. Baskurt, M. C. Santana, and J. Lorenzo-Navarro, "The FG 2015 kinship verification in the wild evaluation," in IEEE International Conference on Automatic Face and Gesture Recognition, 2015, pp. $1-7$.

[115] L. Torresani and K. Lee, "Large margin component analysis," in Advances in Neural Information Processing Systems, 2006, pp. 1385-1392.

[116] J. Hu, J. Lu, Y.-P. Tan, and J. Zhou, "Deep transfer metric learning," IEEE Transactions on Image Processing, vol. 25, no. 12, pp. 5576-5588, 2016.

[117] J. Lu, G. Wang, and P. Moulin, "Localized multifeature metric learning for image-set-based face recognition," IEEE Transactions on Circuits and Systems for Video Technology, vol. 26, no. 3, pp. 529-540, 2016.

[118] J. Lu, G. Wang, W. Deng, and K. Jia, "Reconstruction-based metric learning for unconstrained face verification," IEEE Transactions on Information Forensics and Security, vol. 10, no. 1, pp. 79-89, 2015.

[119] C. Ding, J. Choi, D. Tao, and L. S. Davis, "Multi-directional multi-level dual-cross patterns for robust face recognition," IEEE Transactions on Pattern Analysis and Machine Intelligence, vol. 38, no. 3, pp. 518-531, 2016.

[120] T. Liu and D. Tao, "Classification with noisy labels by importance reweighting," IEEE Transactions on Pattern Analysis and Machine Intelligence, vol. 38, no. 3, pp. 447-461, 2016.

[121] S. Yan, D. Xu, B. Zhang, H. Zhang, Q. Yang, and S. Lin, "Graph embedding and extensions: A general framework for dimensionality reduction," IEEE Transactions on Pattern Analysis and Machine Intelligence, vol. 29, no. 1, pp. 40-51, 2007.

[122] G. Alain and Y. Bengio, "What regularized auto-encoders learn from the data-generating distribution," Journal of Machine Learning Research, vol. 15, no. 1, pp. 3563-3593, 2014.

[123] D. Gray and H. Tao, "Viewpoint invariant pedestrian recognition with an ensemble of localized features," in European Conference on Computer Vision, 2008, pp. 262-275.

[124] W.-S. Zheng, S. Gong, and T. Xiang, "Associating groups of people," in British Machine Vision Conference, 2009, pp. 1-11. 
[125] D. S. Cheng, M. Cristani, M. Stoppa, L. Bazzani, and V. Murino, "Custom pictorial structures for re-identification," in British Machine Vision Conference, 2011, pp. 1-11.

[126] D. Baltieri, R. Vezzani, and R. Cucchiara, "3dpes: 3d people dataset for surveillance and forensics," in Proceedings of the 1st International ACM Workshop on Multimedia access to 3D Human Objects, 2011, pp. 59-64.

[127] L. Zhang, W. Zuo, and D. Zhang, "LSDT: latent sparse domain transfer learning for visual adaptation," IEEE Transactions on Image Processing, vol. 25, no. 3, pp. 1177-1191, 2016.

[128] R. Gopalan, R. Li, and R. Chellappa, "Domain adaptation for object recognition: An unsupervised approach," in IEEE International Conference on Computer Vision, 2011, pp. 999 1006.

[129] B. Gong, Y. Shi, F. Sha, and K. Grauman, "Geodesic flow kernel for unsupervised domain adaptation," in IEEE Conference on Computer Vision and Pattern Recognition, 2012, pp. 2066-2073.

[130] J. Hu, J. Lu, J. Yuan, and Y.-P. Tan, "Large margin multi-metric learning for face and kinship verification in the wild," in Asian Conference on Computer Vision, 2014, pp. 252-267.

[131] J. Hu, J. Lu, Y.-P. Tan, J. Yuan, and J. Zhou, "Local large-margin multi-metric learning for face and kinship verification," IEEE Transactions on Circuits and Systems for Video Technology, 2017, DOI: 10.1109/TCSVT.2017.2691801.

[132] Y. Fu, L. Cao, G. Guo, and T. S. Huang, "Multiple feature fusion by subspace learning," in ACM International Conference on Image and Video Retrieval, 2008, pp. 127-134.

[133] M. Borga, "Canonical correlation: a tutorial," 2001.

[134] M. B. Blaschko and C. H. Lampert, "Correlational spectral clustering," in IEEE Conference on Computer Vision and Pattern Recognition, 2008, pp. 1-8.

[135] A. Sharma, A. Kumar, H. Daume, and D. W. Jacobs, "Generalized multiview analysis: A discriminative latent space," in IEEE Conference on Computer Vision and Pattern Recognition, 2012, pp. 2160-2167.

[136] J. D. M. Rennie and N. Srebro, "Fast maximum margin matrix factorization for collaborative prediction," in International Conference on Machine Learning, 2005, pp. 713-719.

[137] M. Gönen and E. Alpaydin, "Localized multiple kernel learning," in International Conference on Machine Learning, 2008, pp. 352-359.

[138] J. Bohné, Y. Ying, S. Gentric, and M. Pontil, "Large margin local metric learning," in European Conference on Computer Vision, 2014, pp. 679-694.

[139] H. J. Seo and P. Milanfar, "Face verification using the lark representation," IEEE Transactions on Information Forensics and Security, vol. 6, no. 4, pp. 1275-1286, 2011. 
[140] T. Hassner, S. Harel, E. Paz, and R. Enbar, "Effective face frontalization in unconstrained images," in IEEE Conference on Computer Vision and Pattern Recognition, 2015, pp. 42954304.

[141] F. Juefei-Xu, K. Luu, and M. Savvides, "Spartans: Single-sample periocular-based alignment-robust recognition technique applied to non-frontal scenarios," IEEE Transactions on Image Processing, vol. 24, no. 12, pp. 4780-4795, 2015.

[142] X. Zhu, Z. Lei, J. Yan, D. Yi, and S. Z. Li, "High-fidelity pose and expression normalization for face recognition in the wild," in IEEE Conference on Computer Vision and Pattern Recognition, 2015, pp. 787-796.

[143] L. Zheng, K. Idrissi, C. Garcia, S. Duffner, and A. Baskurt, "Triangular similarity metric learning for face verification," in IEEE International Conference on Automatic Face and Gesture Recognition, 2015, pp. 1-7.

[144] H. Li, G. Hua, X. Shen, Z. L. Lin, and J. Brandt, "Eigen-pep for video face recognition," in Asian Conference on Computer Vision, 2014, pp. 17-33.

[145] C. Xiong, L. Liu, X. Zhao, S. Yan, and T.-K. Kim, "Convolutional fusion network for face verification in the wild," IEEE Transactions on Circuits and Systems for Video Technology, vol. 26, no. 3, pp. 517-528, 2016.

[146] O. M. Parkhi, A. Vedaldi, and A. Zisserman, "Deep face recognition," in British Machine Vision Conference, 2015, pp. 41.1-41.12.

[147] Z. Lei, D. Yi, and S. Z. Li, "Learning stacked image descriptor for face recognition," IEEE Transactions on Circuits and Systems for Video Technology, vol. 26, no. 9, pp. 1685-1696, 2016.

[148] Z. Cao, Q. Yin, X. Tang, and J. Sun, "Face recognition with learning-based descriptor," in IEEE Conference on Computer Vision and Pattern Recognition, 2010, pp. 2707-2714.

[149] S. Lazebnik, C. Schmid, and J. Ponce, "Beyond bags of features: Spatial pyramid matching for recognizing natural scene categories," in IEEE Conference on Computer Vision and Pattern Recognition, 2006, pp. 2169-2178.

[150] H. Yan, J. Lu, W. Deng, and X. Zhou, "Discriminative multimetric learning for kinship verification," IEEE Transactions on Information Forensics and Security, vol. 9, no. 7, pp. 1169$1178,2014$. 